\title{
MINIX4RT: A Real-Time Operating System Based on MINIX
}

\author{
Pablo Andrés Pessolani
}

\begin{abstract}
Tanenbaum's MINIX Operating System was extended with a Real-Time microkernel and services to conform MINIX4RT, a Real-Time Operating System for academic uses that includes more flexible Interprocess Communications facilities supporting basic priority inheritance protocol, a fixed priority scheduler, timer and event driven interrupt management, statistics and Real-Time metrics gathering keeping backward compatibility with standard MINIX versions.
\end{abstract}

Keywords: Operating Systems, Minix, Interrupt Handling, Real-Time.

Presented to the Universidad Nacional de La Plata of the Requirements for the Degree of Master on Computer Networks.

February 2006

Thesis Director: Ph.D. Silvio Gonnet

Thesis Co-Director: Eng. Armando De Giusti 
This thesis is dedicated to my lovely daughters Flor and Vicky, to the "love of my life" Ana, and to my "iron willpower" parents Merce and Pety. 


\section{Acknowledgments}

I would like to express my sicere gratitude to Dr. Silvio Gonnet for his suggestions, dedication and vast patience as thesis director.

I would like to thanks Nicolás Cesar and Felipe Ieder for their contributions with the system latency and timeliness tests and German Maglione for supplying the IBM Thinkpad 370 notebook used in the implementation and tests of MINIX4RT.

I acknowledge help received from Telecom Argentina S.A. for sponsoring my UNLP Master career, the Department of Information Systems of UTN-FRSF for sponsoring the presentation of articles about MINIX4RT in the Argentine Symposium of Technology 2004/5 and the Postgraduate Office of UNLP for its attention and patience.

This acknowledgment would be incomplete if I forgot to mention my whole family for their love, support, and encouragement throughout this course of study. 


\section{PREFACE}

MINIX4RT is the result of fifteen years dedicated to study, to research and to teach about Operating Systems. It is a new open-source Real-Time Operating System intended as teaching tool, but it can be usable as a serious system on resource-limited computers.

MINIX4RT is a Real-Time branch of the popular MINIX used in grade level Operating Systems courses. This work discuss the modifications that have been made to MINIX that give it the ability to support the stringent timing requirements of Real-Time applications, while still giving Non Real-Time ones access to the full range of MINIX services without any changes.

MINIX4RT does not stops with this thesis. It is the authors intention to conform a team of developers around the world of people interesting in cooperate with the growth and enhancement of MINIX4RT. 


\section{CONTENTS}

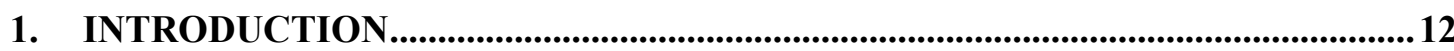

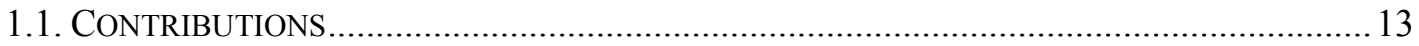

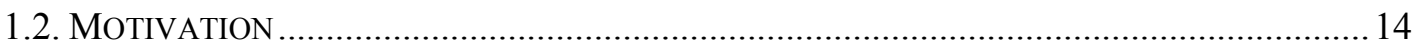

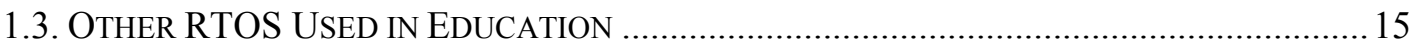

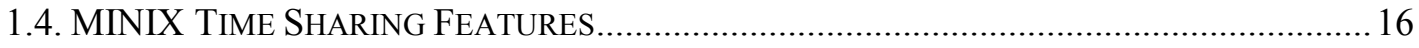

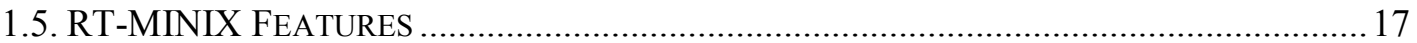

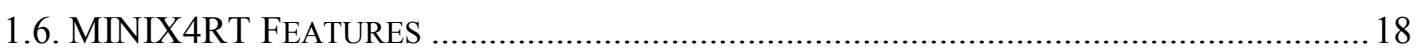

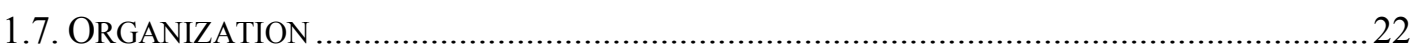

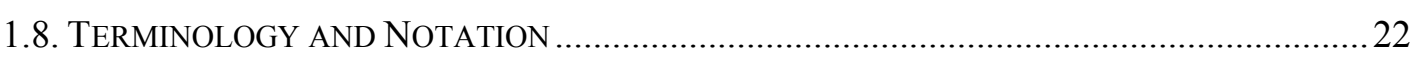

2. ARCHITECTURE AND INTERRUPT HANDLING ..........................................24

2.1. MINIX SYSTEM ARCHITECTURE AND INTERRUPT PROCESSING ....................................24

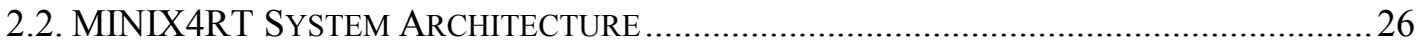

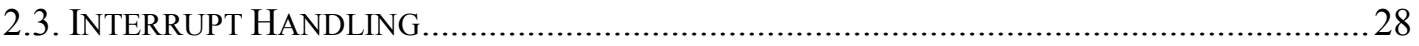

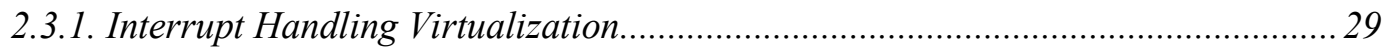

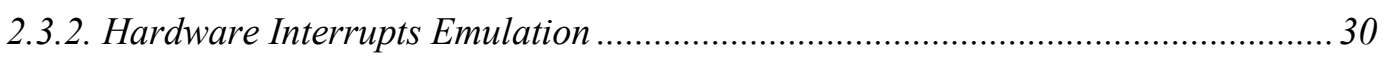

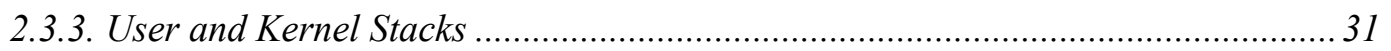

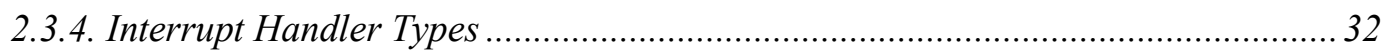

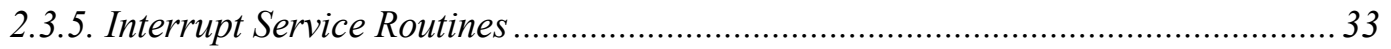

2.3.6. Interrupt Descriptor Data Structure ................................................................... 33

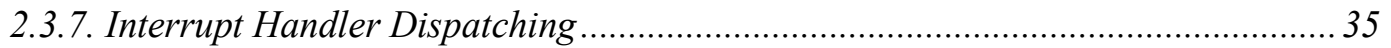

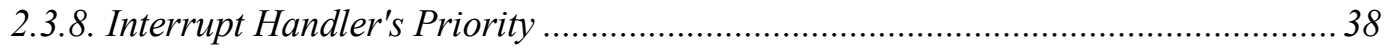

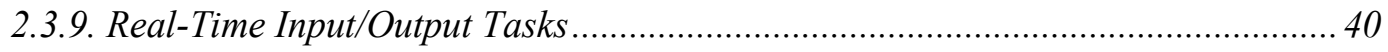

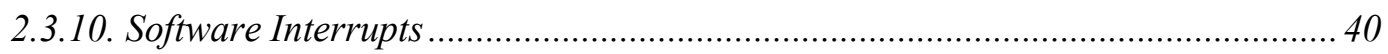

2.3.11. Returning from System Calls and Interrupts Service Routines............................. 41

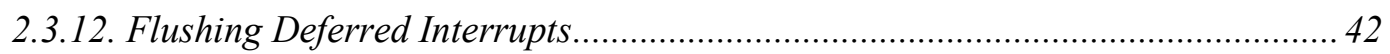

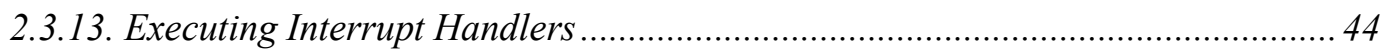

2.3.14. Interrupt Descriptor Timestamp Field ................................................................ 46

2.3.15. Kernel Functions for Interrupt Handling and Synchronization ............................46

2.3.16. Estimating Interrupt Handler Processing Time.................................................. 48

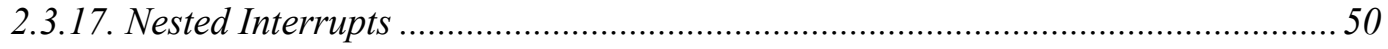

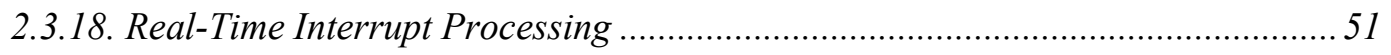

2.3.19. Standard MINIX Non Real-Time interrupts......................................................... 52

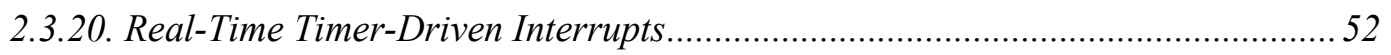

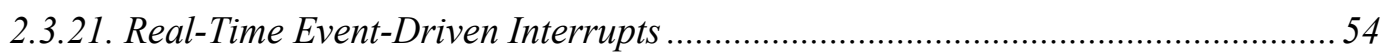

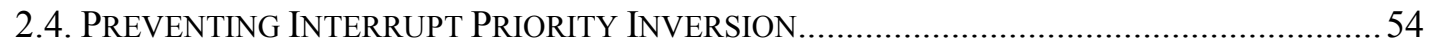


3. RT-PROCESS MANAGEMENT AND SCHEDULING ...............................................57

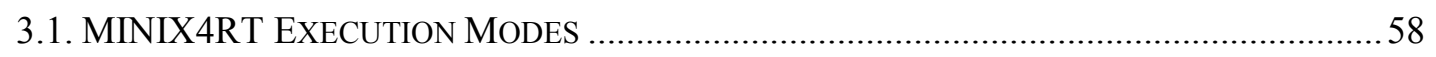

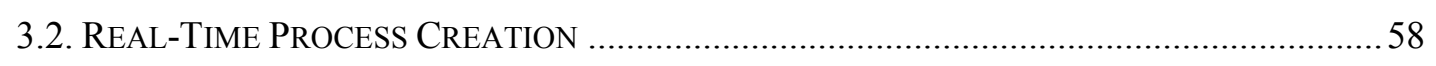

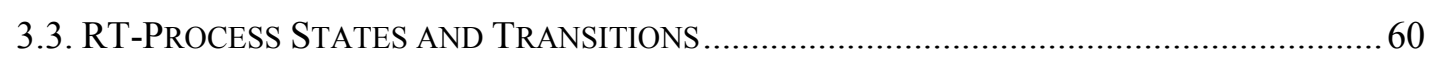

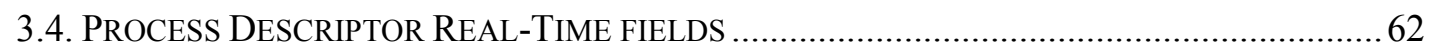

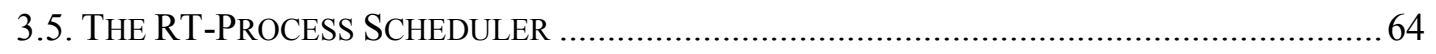

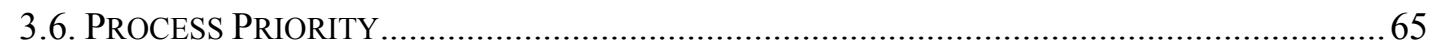

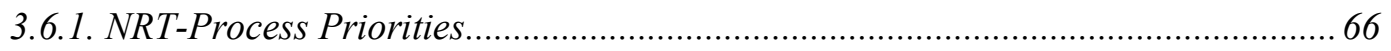

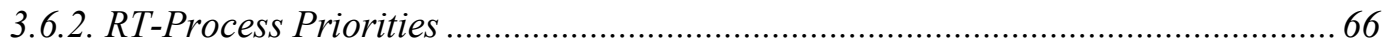

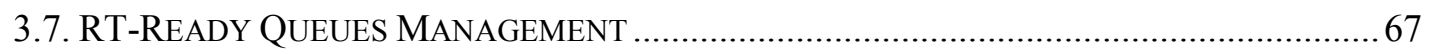

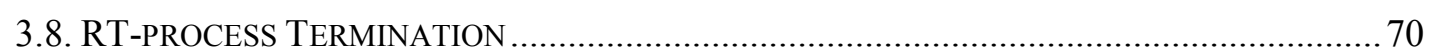

3.8.1. RT-process Termination Using the exit() System Call.......................................... 70

3.8.2. RT-process Termination Using the signal() System Call...................................... 71

3.8.3. Releasing RT-process Resourses and Housecleaning .......................................... 71

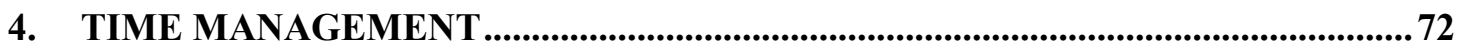

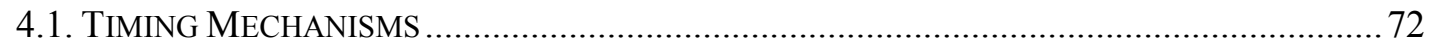

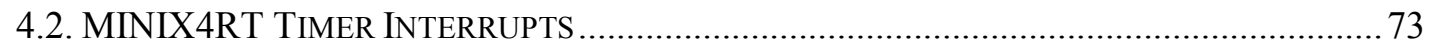

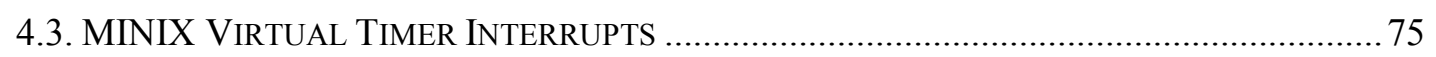

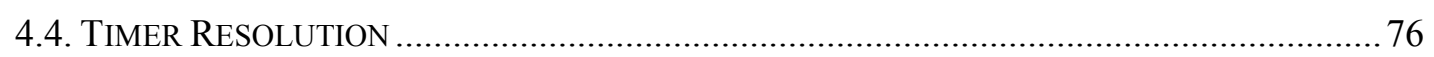

4.5. 8253/4 Programmable InterVAl Timer Programming............................................ 78

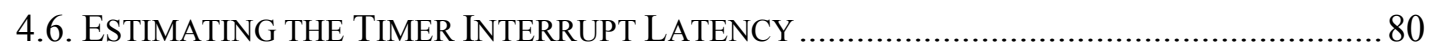

4.7. REAL-TIME AND NON REAL-TIME TIMER HANDLERS.................................................... 81

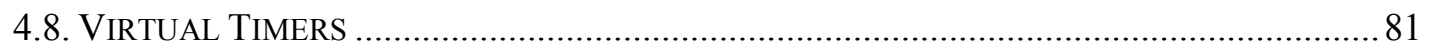

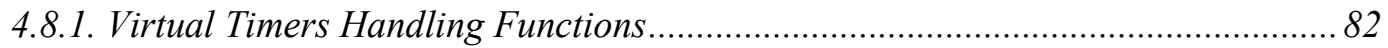

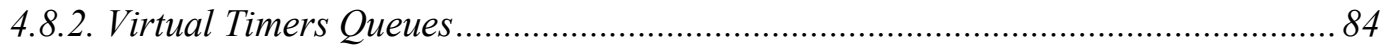

4.8.3. Executing Virtual Timers Actions ................................................................... 85

4.9. VIRTUAL TIMERS HANDLING: OTHER TESTED APPROACHES ........................................ 87

5. REAL-TIME INTERPROCESS COMUNICATION (RT-IPC) ..............................89

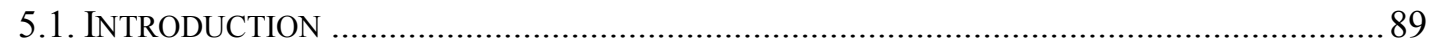

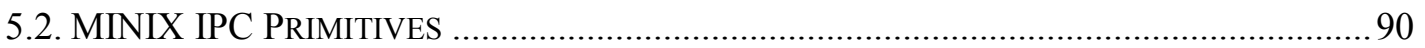

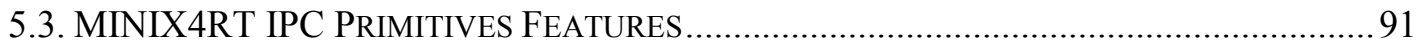

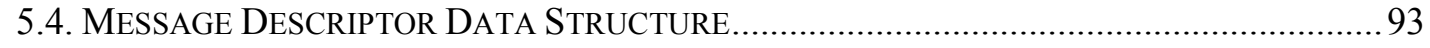

5.4.1. Message Payload Data Structure .......................................................................... 93

5.4.2. Message Header Data Structure …...................................................................... 94 
5.5. The Message QueUe ENTRY DESCRIPTOR .94

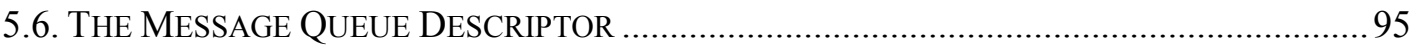

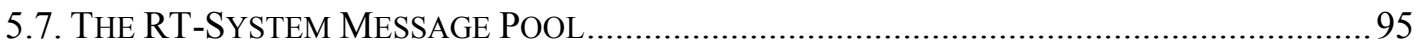

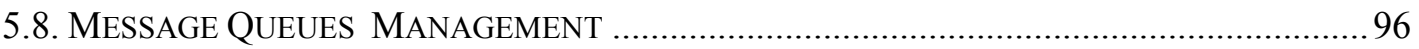

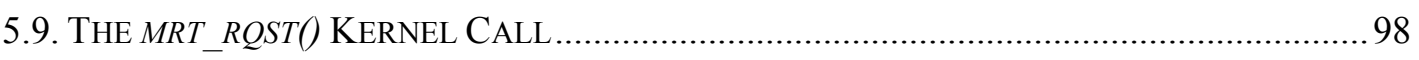

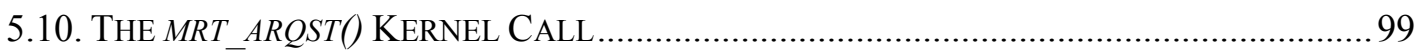

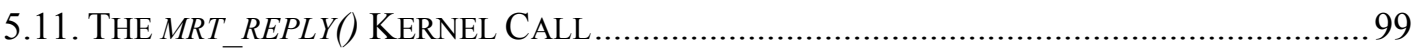

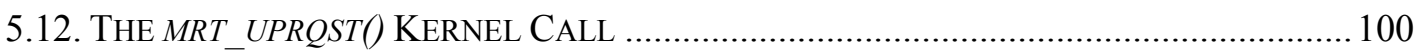

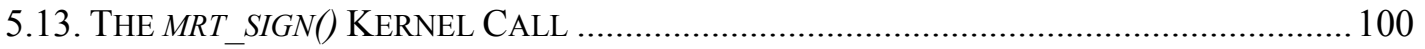

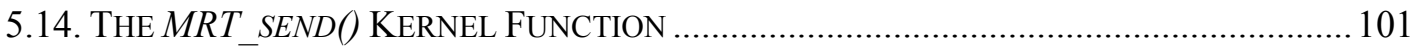

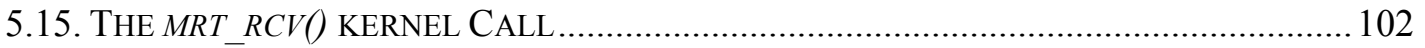

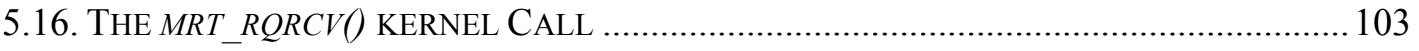

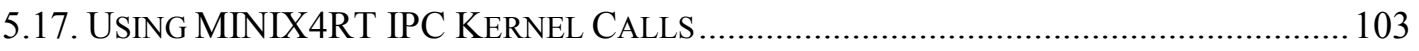

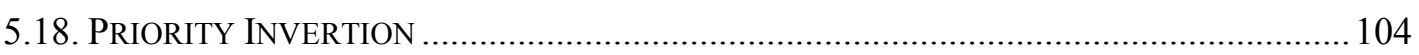

5.19. BASIC PRIORITY INHERITANCE PROTOCOL (BPIP) ...................................................... 106

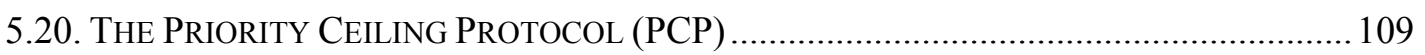

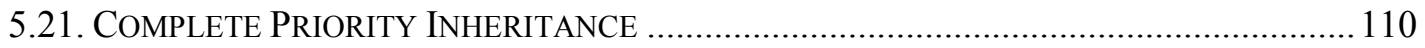

6. RT-SYSTEM CALLS, KERNEL CALLS AND FUNCTIONS................................111

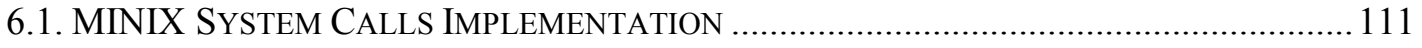

6.2. MINIX4RT SYSTEM CALLS IMPLEMENTATION …....................................................... 112

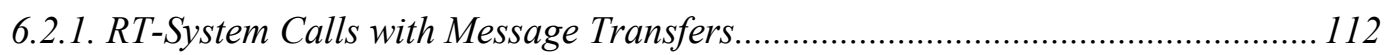

6.2.2. RT-Kernel Calls without Message Transfers...................................................... 113

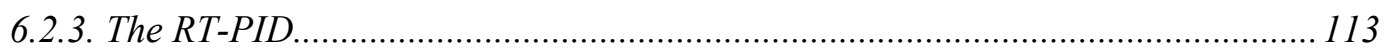

6.3. AdDING NEW RT-SYSTEM CALLS USING MRTTASK ….......................................... 114

6.4. AdDING RT-KERNEL CALLS WITHOUt MESSAGE TRANSFERS ....................................116

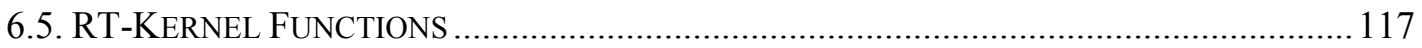

7. REAL-TIME PROCESSING RELATED STATISTICS .............................................120

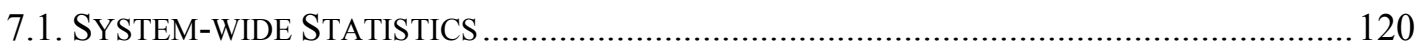

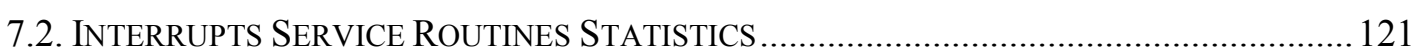

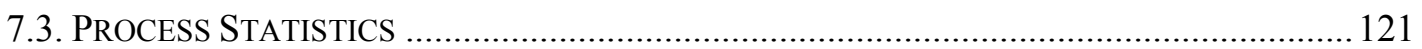

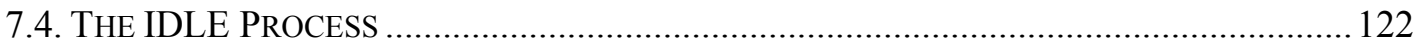

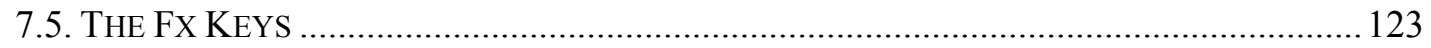

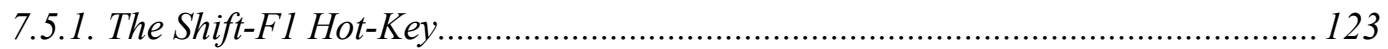

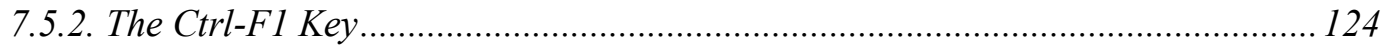

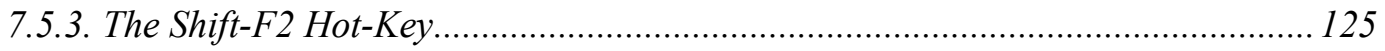


7.5.4. The F4 Key.....

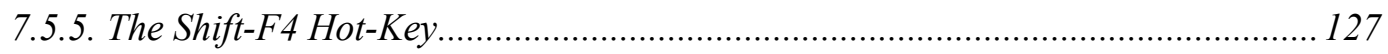

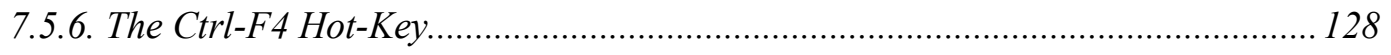

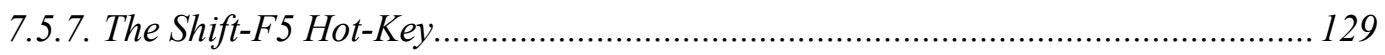

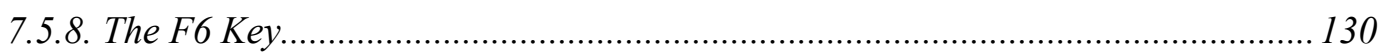

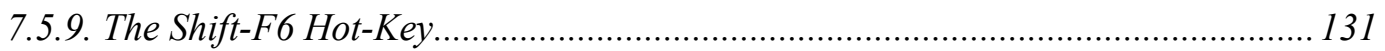

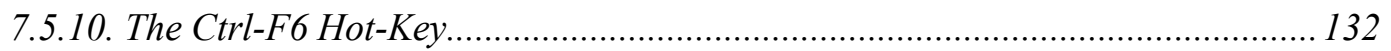

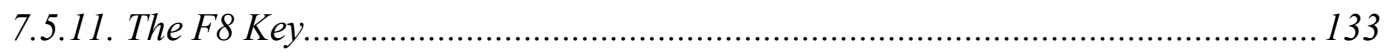

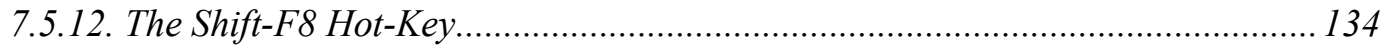

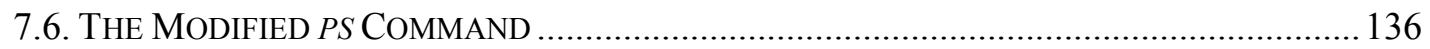

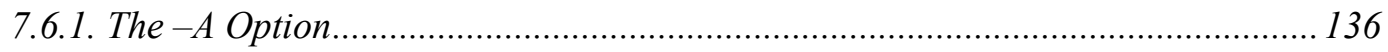

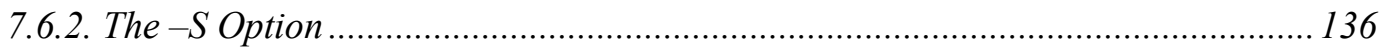

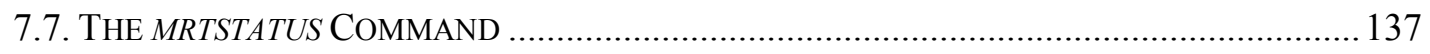

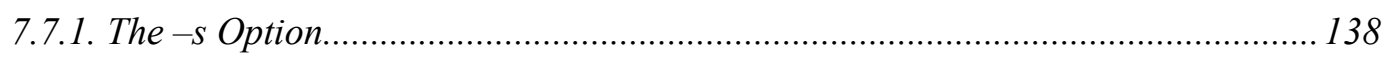

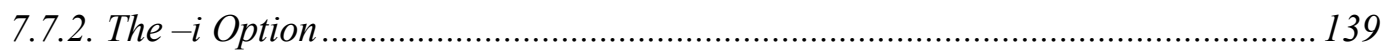

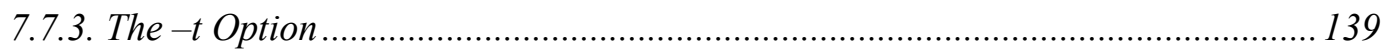

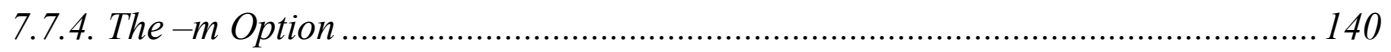

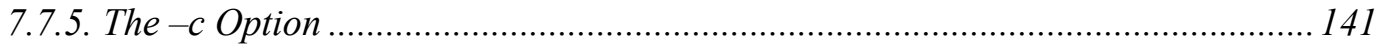

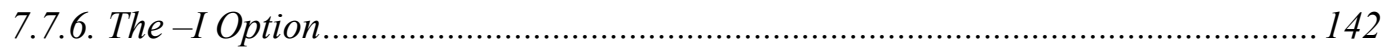

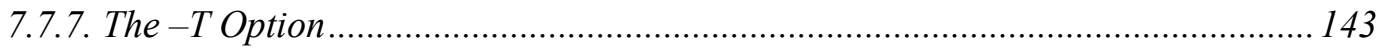

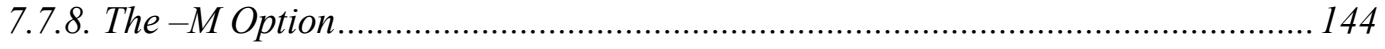

8. CONCLUSIONS AND FUTURE WORKS ..............................................................146

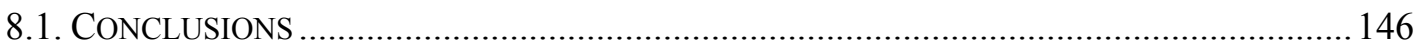

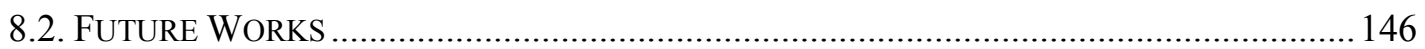

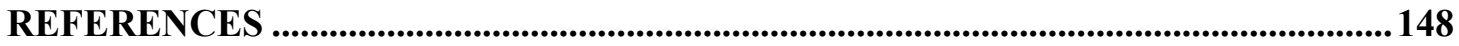

APPENDIX A: RT-SYSTEM CALLS AND RT-KERNEL CALLS REFERENCE ..151

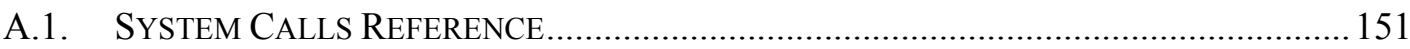

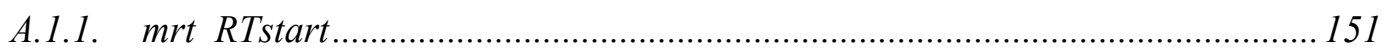

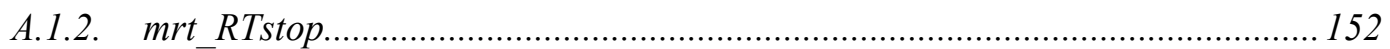

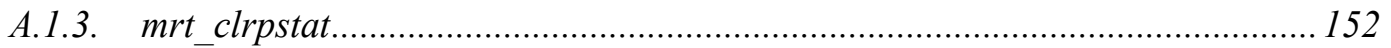

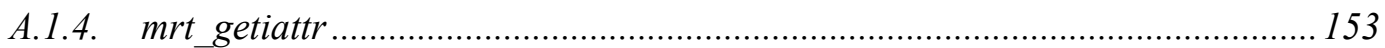

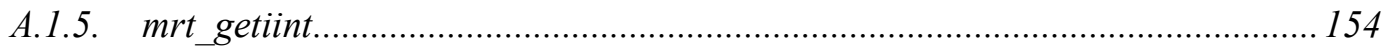

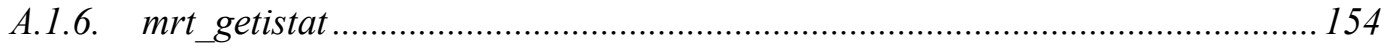

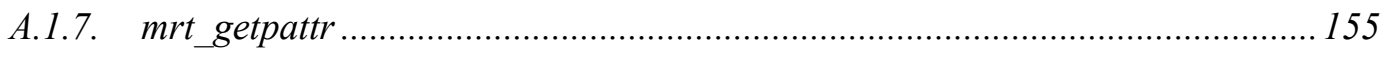

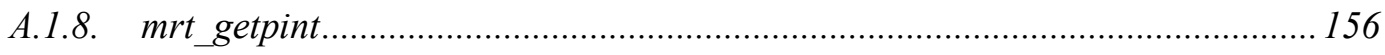




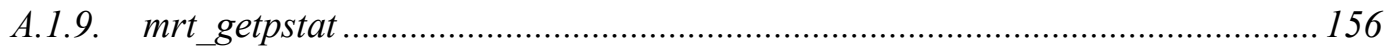

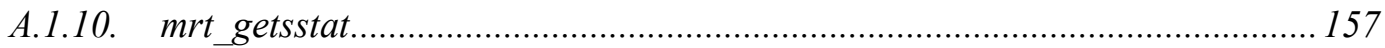

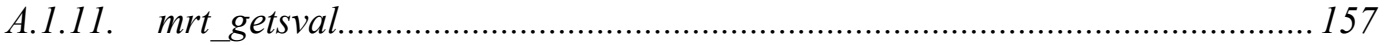

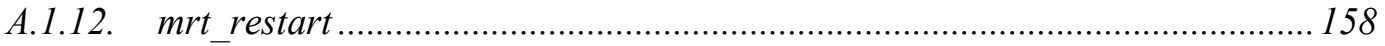

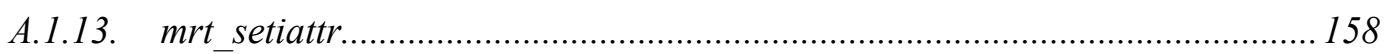

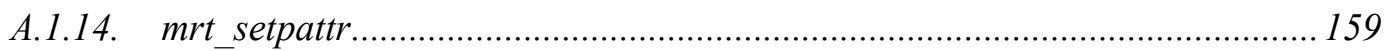

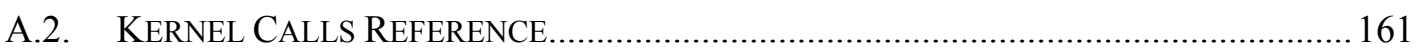

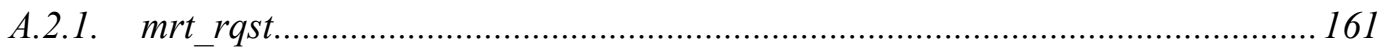

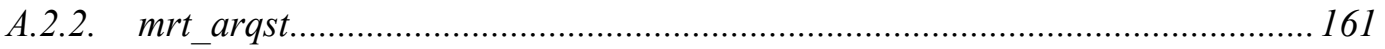

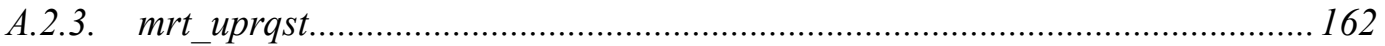

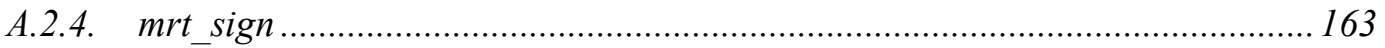

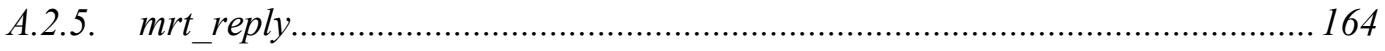

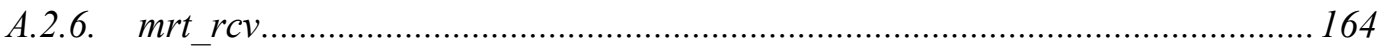

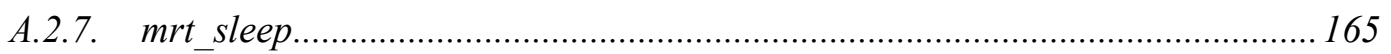

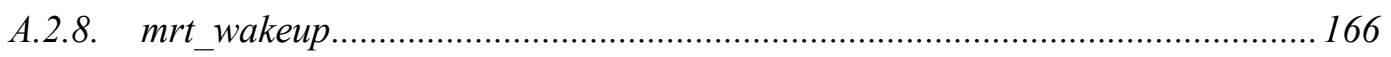

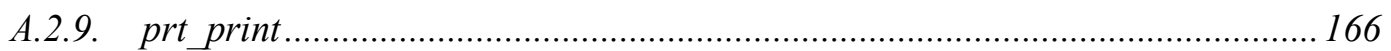

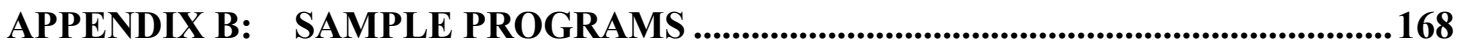

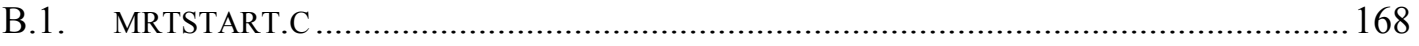

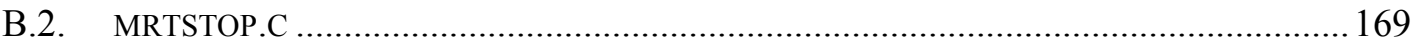

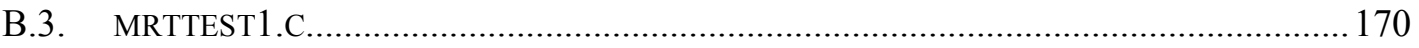

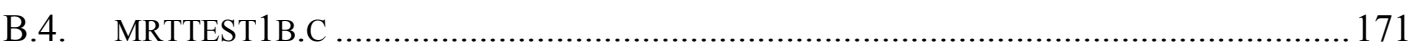

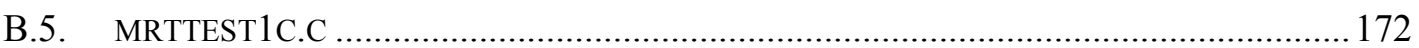

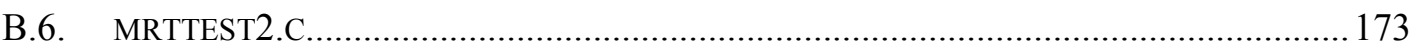

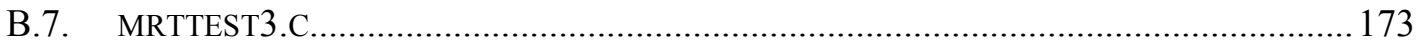

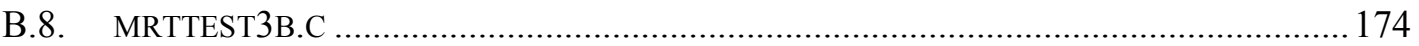

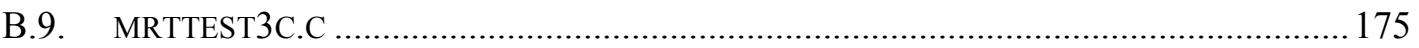

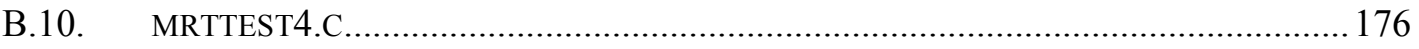

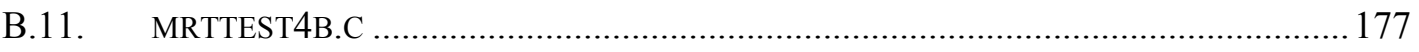

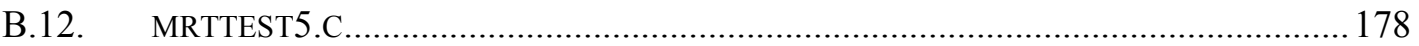

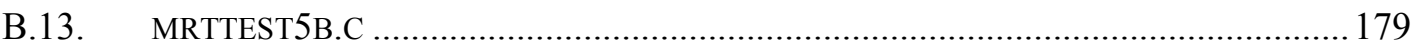

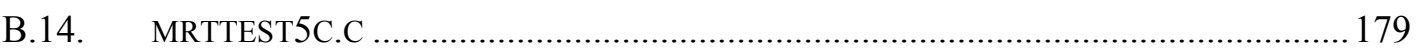

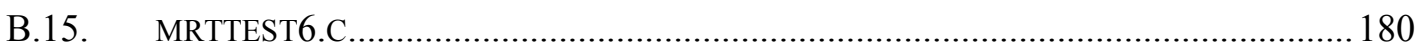

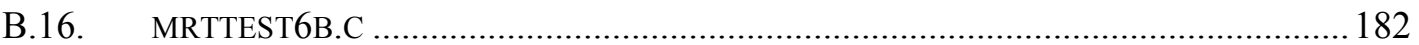

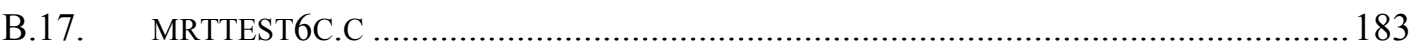

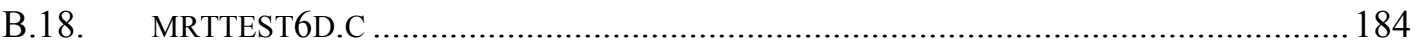

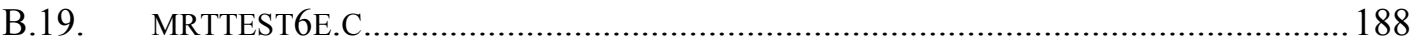




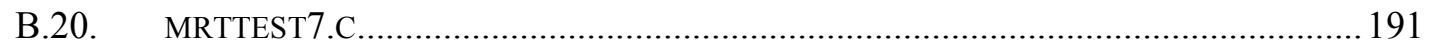

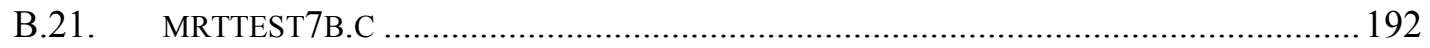

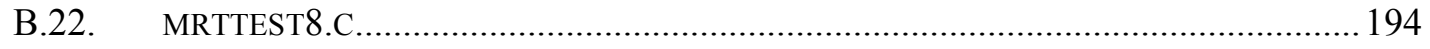

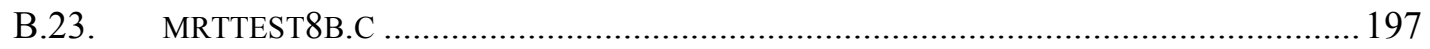

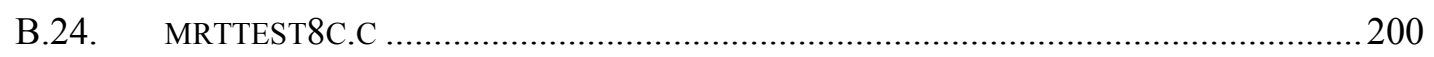

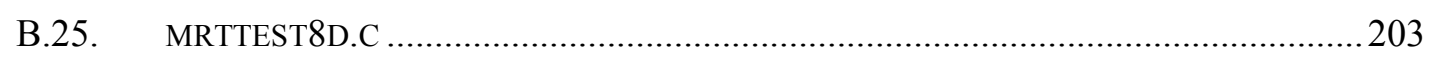

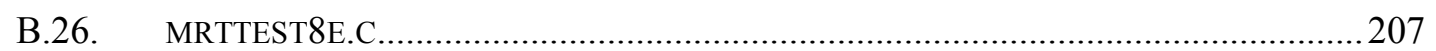

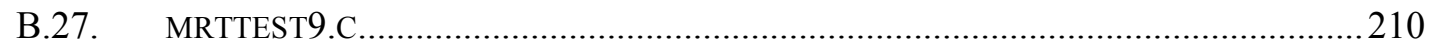

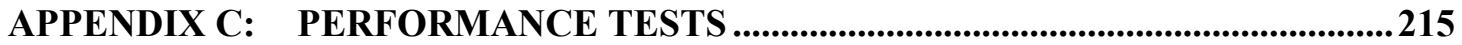

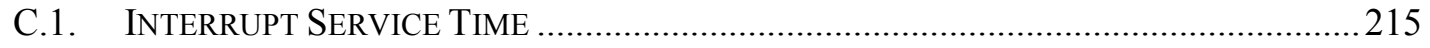

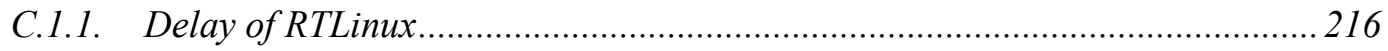

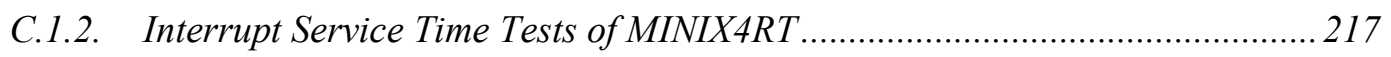

C.1.3. Interrupt Service Time of Software Interrupts................................................2 218

C.1.4. Interrupt Service Time of Event-Driven Interrupts...........................................220

C.1.5. Interrupt Service Time of Timer-Driven Interrupts ......................................... 222

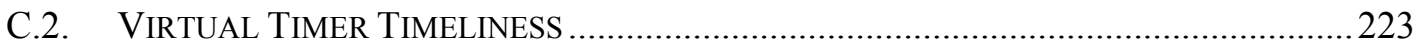

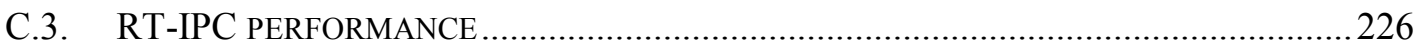

C.3.1. Synchronous Message Transfers Tests without BPIP and without Timeouts ...226

C.3.2. Asynchronous Message Transfers Tests without BPIP and without Timeouts. 227

C.3.3. Synchronous Message Transfers Tests with BPIP and without Timeouts ........ 228

C.3.4. Asynchronous Message Transfers Tests with BBIP and without Timeouts ...... 228

C.3.5. Request/Receive Tests with BPIP and without Timeouts................................2228

C.3.6. Synchronous Message Transfers Tests with BPIP and with Timeouts ............. 229

C.3.7. Asynchronous Message Transfers Tests with BBIP and with Timouts .............2230

C.3.8. Test Request/Receive Tests with BPIP and with Timeouts .............................231

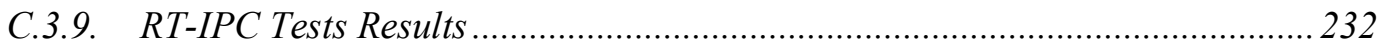

APPENDIX D: SYSTEM DATA STRUCTURES …........................................................233

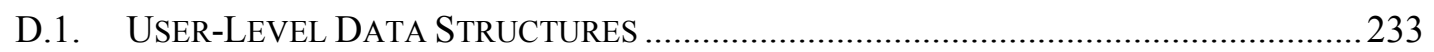

D.1.1. System-wide Data Structures.............................................................................. 233

D.1.2. Interrupt-related Data Structures................................................................... 233

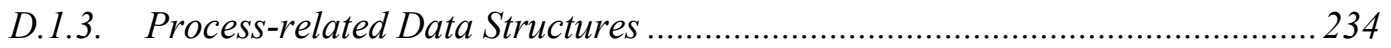

D.1.4. Kernel Calls-related Data Structures.........................................................2235

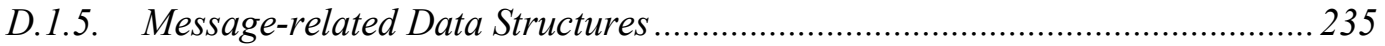

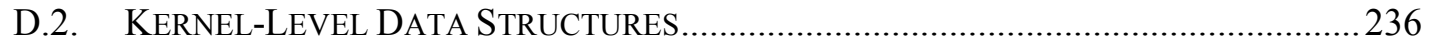

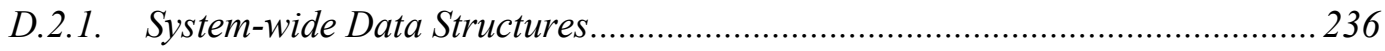




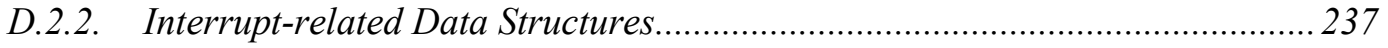

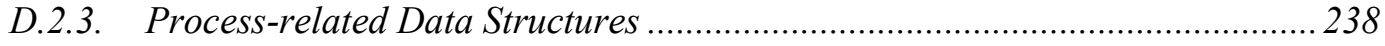

D.2.4. Message-related Data Structures …………...............................................2239

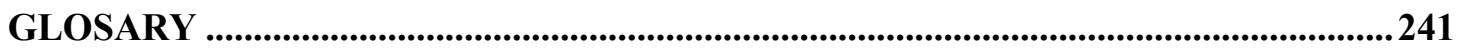




\section{INTRODUCTION}

A Real-Time Operating System (RTOS) supports Real-Time applications. Real-Time applications requirements from the Operating System (OS) are much different from those required by non-time constrained time-sharing applications. MINIX4RT satifies the constrains of many of the applications that impose stringent timing demands on their OS with disastrosous consequences resulting from temporal errors.

To assist Real-Time applications designers, RTOS must facilitate efficient interprocess communication and synchronization, a fast interrupt response time, asynchronous Input and Output (I/O) and timing related facilities.

Real-Time applications written to run on RTOS make use of and rely on the following system capabilities [1]:

- A preemptive kernel.

- Fixed-priority scheduling policies.

- Real-Time clocks and timers.

- Asynchronous I/O.

- Queued Real-Time signals.

- Process communication facilities.

Computer science students and professionals working on RTOS need a deep knowledge about every software component and the interactions with hardware devices considering timing constraints. 
RTOS instructors can choose among commercial or free licence software to develop their laboratory practice. Commercially available RTOSs are too costly and proprietary to be used by academic institutions. Free licence and open source RTOSs have been designed with emphasis on predictability as a key design feature with complex source code readability.

The thesis of this work is an academic purpose RTOS designed to present an approach of adding Real-Time facilities to a standard time-sharing OS. The proposed architecture defines a way to schedule, manage, and execute Real-Time processes. The resulting system will be able to guarantee that Real-Time processes will meet their stated deadlines.

The thesis has been proved by building MINIX4RT, a research prototype implementation of that architecture based on MINIX [2], and then testing the implementation by executing periodic and non-periodic Real-Time processes using the added Real-Time facilities.

\subsection{Contributions}

This work makes several contributions to the existing body of knowledge of academic purpose Operating Systems.

- Architecture: Adds a Real-Time sub-kernel below a general-purpose OS.

- Interrupt Management: A detailed description of how interrupts are managed to support Real-Time processing with minimal priority inversion.

- Process Management: It presents a new viewpoint of two domains of process states, transitions and scheduling.

- Time Management: An original approach is proposed to deal with the execution of actions triggered by time that minimize the priority inversion problem.

- System Calls: A detailed description of how System Calls and Kernel Calls are implemented and how new ones can be added.

- Statistics: The prototype has several methods to collect system states, parameters and statistics that are useful for teaching, debugging and system verification. 


\subsection{Motivation}

The goal of the MINIX4RT project is to provide an educational tool for RTOS courses as MINIX $[\underline{3}, \underline{4}, \underline{5}]$ and Linux [ $[\underline{6}]$ do for OS Design and Implementation courses.

The decision of adopting MINIX as foundation for this work is based on:

- Documentation Availability: Tanenbaum and Woodhull book [2] is the main MINIX reference, but plentyful documentation can be found on the Internet as on http://www.minix3.org, http://minix1.hampshire.edu/, etc.

- Hardware Platform: A PC x386 with $16 \mathrm{Mb}$ of RAM and a hard disk of $100 \mathrm{Mb}$ is enough to run MINIX. These modest hardware requirements allow old PCs to be recycled for Real-Time laboratories. Moreover, MINIX can run in emulated environments as BOSCH, VMWare, MS Virtual PC, QEMU etc. (more information about emulated environments in http://minix1.hampshire.edu/hints.html\#emul-virt). This feature allows sharing laboratories among courses without additional manteinance and operational impact.

- Modular and Elegant Design: MINIX is a small UNIX-like operating system, originally developed by Andrew Tanenbaum as a teaching tool for operating systems classes. MINIX was designed with a more modular internal structure than the monolithic UNIX kernel, and this structure affects the way in which new features could be added to MINIX.

- Existing Applications and Programming Tools: The same project goal could be reached writing a RTOS from scratch but this strategy implies the construction of a new user interface to run applications like text editors, compilers, linkers, etc. that does not need Real-Time services. Using MINIX as the interface between the Real-Time Kernel and User-space applications simplifies the development of the system and allows the use of well-known tools.

- Academic Experience: The author teaches about Operating System in Facultad Regional Santa Fe of the Universidad Tecnológica Nacional (Argentine), where MINIX is used as an academic tool since 1993. 
MINIX4RT implementation focus on source code readability (perhaps sacrificing performance) to allow instructors to easily do a multiplicity of grade courses assignments, laboratory tests and other academic uses with an open source RTOS. Some interesting projects could be:

- Adding new functionalities and System Calls to MINIX4RT.

- Coding and Testing Real-Time scheduling algorithms.

- Coding Servers to handle Real-Time Aperiodic tasks.

- Porting hard Real-Time network protocol stacks as RTNET [7] or RETHER []].

- Building Remote Device Drivers to control Robots.

- Build embedded RTOS based on MINIX4RT.

- Add new features and System calls to MINIX4RT to be compliant with the IEEE POSIX 4 standard.

Plentyful statistics are gathered to make the RTOS more educational about its operation and helpful for debbuging applications.

\subsection{Other RTOS Used in Education}

Most RTOS used in education like RTLinux [9], RT-Mach [1], QNX [11], and RTMINIX $[12,13]$ are suitable for Real-Time Systems courses but this fact must not be confused with teaching how a RTOS works. Those systems are focused on performance, schedulability, research, commecial market, etc. but not for academic purposes. Their source code readability (if they are available), complex algorithms and limited documentation do not help for students understanding.

Until the development of MINIX4RT, RTLinux was the system used in laboratory practice and assignments of the Advanced Operating System course in Facultad Regional Santa Fe of the Universidad Tecnológica Nacional (Argentine). Even RTLinux is used in education, its kernel source code is not well documented and requires a deep knowledge of the constant growing Linux kernel. As basic courses of Operating Systems in Facultad Regional Santa Fe are based on MINIX, MINIX4RT appears as the natural choice for practice in a RTOS course. 
The major algorithms and data structures used by MINIX4RT were created or adapted trying to achieve a balance between efficiency and simplicity inspired in popular OS as:

- RTLinux [9]: The Virtual Machine (VM) concept limited to interrupt emulation.

- RT-MACH [10]: RT-Inter Process Communications.

- QNX [11]: Priority queues.

- Windows NT[14] : Interrupt queues.

- Linux [15]: Virtual timers.

- MACH [16]: Message queues.

\subsection{MINIX Time Sharing Features}

MINIX [2] is a complete, time-sharing, multitasking OS developed from scratch by Andrew S. Tanenbaum. It is a general-purpose OS broadly used in Computer Science degree courses.

Though it is copyrighted, the source has become widely available for universities for studying and research. Its main features are:

- Microkernel based: Provides process management and scheduling, basic memory management, interprocess communication, interrupt processing and low level I/O support.

- Multilayer system: Allows for modular design and clear implementation of new features.

- Client/Server model: All system services and device drivers are implemented as server processes with their own execution environment.

- Message Transfer Interprocess Communications (IPC): Used for process synchronization and data sharing.

- Interrupt hiding: Interrupts are converted into message transfers. 


\subsection{RT-MINIX Features}

Wainer and Rogina $[\underline{12}, \underline{13}]$ changed MINIX OS to support RT-processing and named it "RTMINIX". Its main features are:

- Scheduling Algorithms Selection: Rate Monotonic (RM) and Earliest Deadline First (EDF) scheduling were included. These strategies were later combined with other traditional strategies, such as Least Laxity First, Least Slack First and Deadline Monotonic.

- Joined Scheduling Queues: Process execution priority was implemented using a multiqueue scheme to accommodate Real-Time processes along with interactive and CPU-bound tasks.

- Real-Time Metrics collection: Several variables about the whole operation are accessible to the user to provide data for benchmarking and testing new developments.

- Timer Resolution Management: The resolution of the Timer can be changed to get better accuracy while scheduling processes.

Several data structures in the OS were modified to consider processes period, execution time and criticality. But RT-MINIX does not have its own architecture, it is like a patch for MINIX in order to provide the user with a set of System Calls to create and manage periodic or aperiodic processes. That approach implies some academic and functional limitations because:

- It does not have its own architecture: The source code of kernel functions and fields of data structures that treat with Real-Time issues are merged with those that treats with non Real-Time ones.

- It does not serve hardware interrupts in priority order: This fact could produce unbounded priority inversion. While a higher priority interrupt handler is running, lower priority interrupts could be attended increasing the interference.

- It has only one level of priority for all Real-Time processes: This fact reduces the system schedulability. Even worst, MINIX Tasks and Servers have higher priorities than Real-Time processes. While a RT-process is 
running, a standard MINIX Task or Server could preempt it. This is another case of unbounded priority inversion.

- It uses standard MINIX message transfers as its IPC primitives: MINIX use FIFO discipline to receive messages from several processes and this implies that a priority inversion problem is present.

- It does not have any protocol against unbounded priority inversion: Its utilization in projects that use cooperating processes is limited.

- Increasing the Timer resolution also increases the system overhead: To increase the Timer resolution, RT-MINIX increases the Timer frequency. This strategy executes MINIX Timer interrupt handler at higher frequency, thus increasing the system overhead.

- A Real-Time process can use MINIX System Calls: When MINIX Server receives a request from a Real-Time process it will be executed on behalf of it, but without any Real-Time attributes.

- MINIX Timer handler was modified to support RT-alarms: As MINIX scheduler treats Tasks in FIFO order, other MINIX Tasks could be executed before the Timer Task increasing the RT-alarms latency.

RT-MINIX defines a new set of signals to indicate special situations, such as missed deadlines, overload or uncertainty of the schedulability of the set of processes.

\subsection{MINIX4RT Features}

Existing RTOS can be divided in two categories:

- Systems implemented using somewhat stripped down and optimized (or specialized) versions of conventional time-sharing OS.

- Systems starting from scratch, focusing on predictability as a key design feature.

MINIX4RT design is based on the former category using MINIX as the conventional OS. It offers a predictable RT-computing environment at a lower cost than proprietary RTOS used for academic purposes. Furthermore, the same applications and tools used to edit text files, compile 
programs, list directories contents, etc, which run on MINIX, run without any change on MINIX4RT. This is a important benefit because there is no need to migrate or cross-compile applications.

The following are desirable characteristics in a RTOS:

- Small kernel size: This it will enable the operating system to be used on embedded systems.

- Low context-switching overheads: This reduce the process activation latency and increase system schedulability.

- Fast interrupt service: Often, RTOS respond to external systems through an interrupt mechanism, therefore a fast interrupt service make the system more responsive.

- User defined process scheduling: A process could be schedulable periodically, or triggered by an event, or at specified time, etc. It is desirable that the user could select the process scheduling that best fit for his application.

- Provision for user definable priorities: The schedulability of a RTOS increase with higher numbers of priority levels that the user can define into his applications.

- Provision for specification of deadlines: The deadline is an important processing parameter of Real-Time applications. Some scheduling algorithms use processes' deadlines to assign priorities (i.e. Deadline Monotonic Scheduling). The deadline is used in critical situations where a watchdog monitoring process must be activated when the process could not meet it deadline.

Most of the characteristics described above are fulfilled by MINIX4RT considered in a academic environment.

The major features of MINIX4RT are summarized as follows:

- Layered Architecture: As it is explained in Chapter 2, MINIX4RT has a layered architecture that helps to change a component without affecting the others. 
- Real-Time Sub-kernel: A Real-Time micro-kernel that deals with interrupts, IPC, time management and scheduling is installed below MINIX kernel. The advantage of using a microkernel for RTOS is that the preemptability is better, the size of the kernel becomes much smaller, and the addition/removal of services is easier.

- Timer/Event Driven Interrupt Management: Device Driver writers can choice among two strategies of Real-Time Interrupt management.

- Fixed Priority Hardware Interrupt Processing: A priority can be assigned to each hardware interrupt that let service then in priority order.

- Two Stages Interrupt Handling: Interrupt can be serviced in two stages. The hardware interrupt handler (inside interrupt time) performs the first part of the needed work and a software Interrupt handler (outside interrupt time) does the remaining work.

- Fixed Priority Real-Time Scheduling: Each process has an assigned priority. The RT-kernel schedules them in priority order with preemption.

- Periodic and Non-Periodic RT-processing: A period can be specified for a periodic process; the Real-Time kernel schedules it on period expiration.

- Synchronous/Asynchronous Message Transfer using Message Queues: The added RT-kernel offers a new set of Real-Time IPC primitives based on Message Queues.

- Priority Based Message Queue Discipline: A priority based discipline could be specified on each Message Queue for message dequeueing.

- IPC with Basic Priority Inheritance Protocol support: To avoid the unbounded priority inversion problem among communicating processes (explained in Chapter 5).

- Receive and Synchronous Send Timeout Support: To avoid deadlocks and detect dead processes 
- Timer Resolution Management Detached from MINIX Timer: A Timer interrupt of $50 \mathrm{~Hz}$ is emulated for the MINIX kernel eventhough the hardware Timer interrupt has a higher frequency.

- Process and Interrupt Handlers Deadline Expiration Watchdogs: The use of watchdog processes is a common use strategy to deal with malfunctioning RT-processes. When a process does not perform its regular function in a specified time (deadline) another process (watchdog) is signaled to take corrective actions.

- Software Timers: They are system facilities used for time related purposes as alarms, timeouts and periodic processing, etc. One particular feature of MINIX4RT is that it handles software timer actions in priority order.

- Statistics and Real-Time Metrics: There are several facilities to gather information about the system status and performance (detailled in $\underline{\text { Chapter }}$ 7).

It is widely believed that microkernel based systems are inherently inefficient and a multilayer message transfer kernel has a performance disadvantage when compared with monolithic kernel. But [17] presents evidence that inefficiency is not inherited from the basic idea but from improper implementation.

MINIX4RT provides the capability of running Real-Time processes and MINIX processes on the same machine. These Real-Time processes are executed when necessary no matter what MINIX is doing.

The Real-Time microkernel works by treating the MINIX OS kernel as a task been executed under a small RTOS based on software emulation of interrupt control hardware. In fact, MINIX is like the idle process for the Real-Time microkernel been executed only when there are no Real-Time processes to run. When MINIX tells the hardware to disable interrupts, the Real-Time microkernel intercepts the request, records it, and returns to MINIX. If one of these "disabled" interrupts occurs, the Real-Time microkernel records its occurrence and returns without executing the MINIX interrupt handler. Later, when MINIX enables interrupts, all handlers of the recorded interrupts are executed.

MINIX4RT can handle devices in two ways: 
- Event Driven (ED): An ED-interrupt handler is executed when the Hardware Interrupt occurs or its execution is delayed until its priority will be greater than the priority of the current process.

- Timer Driven (TD): A TD-interrupt handler is executed only on the expirations of its specified period.

The current version of MINIX4RT is based on version 2.0.2 for 32 bits INTEL [18] processors of MINIX; and thus it requires the same hardware platform.

\subsection{Organization}

The thesis is organized as follows. Chapter 2 describes Architecture and Interrupt Handling topics on MINIX, RT-MINIX, and MINIX4RT. Chapter 3 present details of Real-Time Process Management and Scheduling. Chapter 4 contains a discussion about Time Management. Chapter 5 is describes Real-Time Interprocess Communication (RT-IPC), priority inversion and the priority inheritance protocol. Chapter 6 provides a detailled explanation of Real-Time System Calls, Kernel Calls and Kernel Functions. Chapter 7 is devoted to Real-Time Processing Related Statistics. Chapter $\underline{8}$ describes future works and summarizes the results of this work.

At the end of this document, a reference of MINIX4RT System Calls and Kernel Calls can be found in Appendix A. Appendix B presents several sample programs. Appendix C describes the set of tests carried out on the Real-Time system and shows its results and Appendix D shows main system data structures.

This thesis does not cover neither topics about Memory Management, File System Management nor Network Managment because MINIX services are used for these issues.

\subsection{Terminology and Notation}

In Real-Time terminology, a Task is the term often used for a process, but in MINIX terminology, a Task refers to a special process type used in the implementation of MINIX device drivers.

Other confusing term in computer science is the IBM-compatible Personal Computer (PC) device that can produce interrupts at regular periods (ticks). MINIX routines refer to it as the Clock, but Timer is the correct term for that device. 
From here, Real-Time related words will be preceded by "RT-" prefix and Non Real-Time related words will be preceded by "NRT-" prefix.

Additional terms used in this document are included in the Glossary to clarify terminology and notation. 


\section{ARCHITECTURE AND INTERRUPT HANDLING}

This chapter reviews some MINIX background information, its architecture and interrupt processing needed to understand the MINIX4RT design approch. Also, it give the details of the MINIX4RT architecture, how interrupt virtualization is accomplished, the type of interrupt handling that systems programmers could choose to attend devices, and some considerations about priority inversion, a common problem presents in the design of RT-systems.

\subsection{MINIX System Architecture and Interrupt Processing}

MINIX is a collection of processes that communicate with each other and with User-level processes using message passing. This design results on a modular and flexible architecture, making it easy to replace one component without having even to recompile other modules[2].

MINIX is structured in four layers as it can see in Figure 2.1.

- Layer 1: The kernel that provides context switching, process scheduling, interrupt handling, basic memory management and IPC.

- Layer 2: Tasks that handle low level I/O operations.

- Layer 3: Server processes that handle System Call services with a Memory Manager server (MM) and a File System server (FS) (other servers could be added).

- Layer 4: The User-level processes such init, shells, compilers, editors, etc.

Each layer only communicates with the ones immediately above and below through the message passing primitives that scale very easily to distributed systems. Therefore, There is not 
difficult to modify or to add new components without breaking what already works making it a good choice for teaching the design and implementation of an OS.

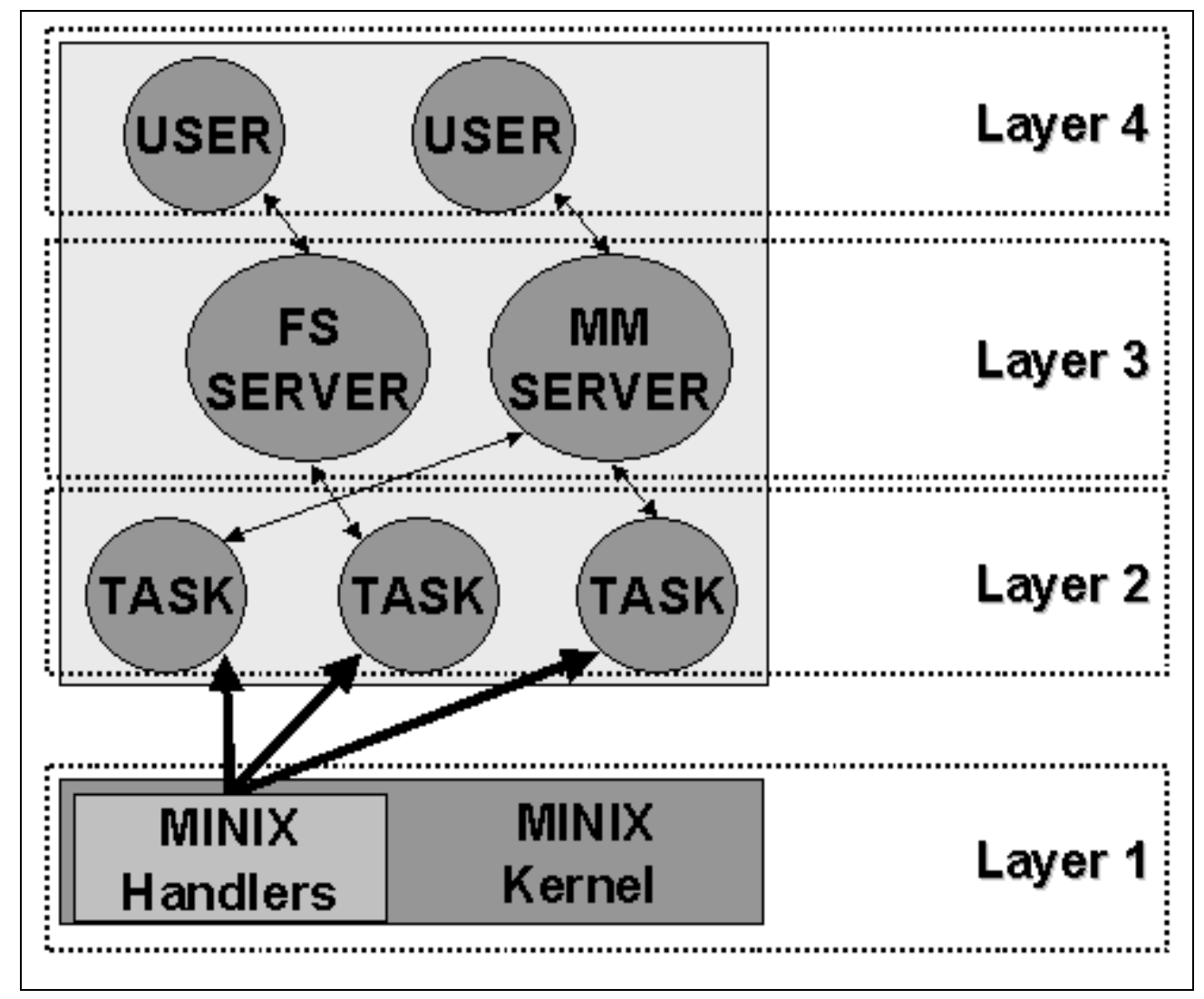

Figure 2.1: MINIX Architecture.

MINIX message passing primitives have some constraints:

- All messages have fixed and small sizes.

- The queue of waiting processes is in the process table.

- User processes can only communicate with Servers, which in turn communicate with I/O Tasks.

- Usually, an application does not construct messages by itself, this is accomplished by the System Calls library code.

Message passing is also used by the kernel to hide hardware interrupts. An interrupt is usually defined as an event that alters the sequence of instructions executed by a processor [15]. In MINIX, when a hardware device interrupts the CPU, an interrupt handler is called, but if more time is needed to complete the job, the handler sends a message to the device Task calling the scheduler on exit. As the scheduler gives I/O Tasks greater priority than User-level processes and Servers, the device Task 
is executed to resume the interrupt service out of interrupt time. This approach is often called two stages interrupt handling.

An I/O Task is like a kernel thread that share kernel address space but it has its own processing attributes. The use of an I/O Task to complete the interrupt processing performs well enough in a time sharing environment but can introduce unbounded delay in RT-processing. Two factors affects the interrupt service response time:

1. MINIX scheduler uses three priority queues, one for I/O Tasks, one for Server processes and one for User-level processes. As each queue is arranged in FIFO order, it is not suitable to be used in time constrained systems where a priority order is needed.

2. MINIX hides interrupts using message transfers. It is very common that on each hardware interrupt the kernel sends a message to an I/O Task. This fact forces a context switch before running the Task increasing the system latency and reducing the schedulability of RT-processes.

A key component in the Intel $\mathrm{x} 86$ hardware architecture is the Interrupt Descriptor Table (IDT) [18]. The IDT associates each interrupt or exception vector with the address of the corresponding interrupt or exception handler. The IDT must be properly initialized before the kernel enables interrupts. The IDT is an array of 8 byte interrupt descriptors in memory devoted to specifying (at most) 256 interrupt service routines. The first 32 entries are reserved for processor exceptions and Non-Maskable Interrupts (NMI), the following 16 are assigned to maskable interrupts, that is, to interrupts caused by Interrupt Requests of hardware devices (IRQs). The remaining entries are available for processor traps, that INTEL designates as "software interrupts". MINIX defines a table called irq_table[] that has function pointers to interrupt handlers codified using the $\mathrm{C}$ programming language.

\subsection{MINIX4RT System Architecture}

As MINIX4RT intends to be used in an academic environment, its design has been done to be as least intrusive as possible in the standard MINIX source code. Yodaiken and Barabanov [9] have proposed a separate, small, RT-kernel between the hardware and Linux (often called a sub-kernel) for RTLinux. The key idea is how interrupt management is done. As result, one RTOS hosts a standard time sharing OS . Those OSs have their own sets of System Calls. 
MINIX4RT follows RTLinux approach where MINIX4RT hosts the standard MINIX time sharing OS. The left side of Figure 2.2 shows the MINIX OS framed by a dotted line, supported by the MINIX4RT kernel. At the right side of Figure 2.2 shows RT-handlers, RT-tasks and RT-processes also supported by the RT-kernel. At the center of Figure 2.2, there is a Task named MRTTASK that function as a glue among MINIX applications and the RT-kernel (explained in $\underline{\text { Chapter 6). }}$.

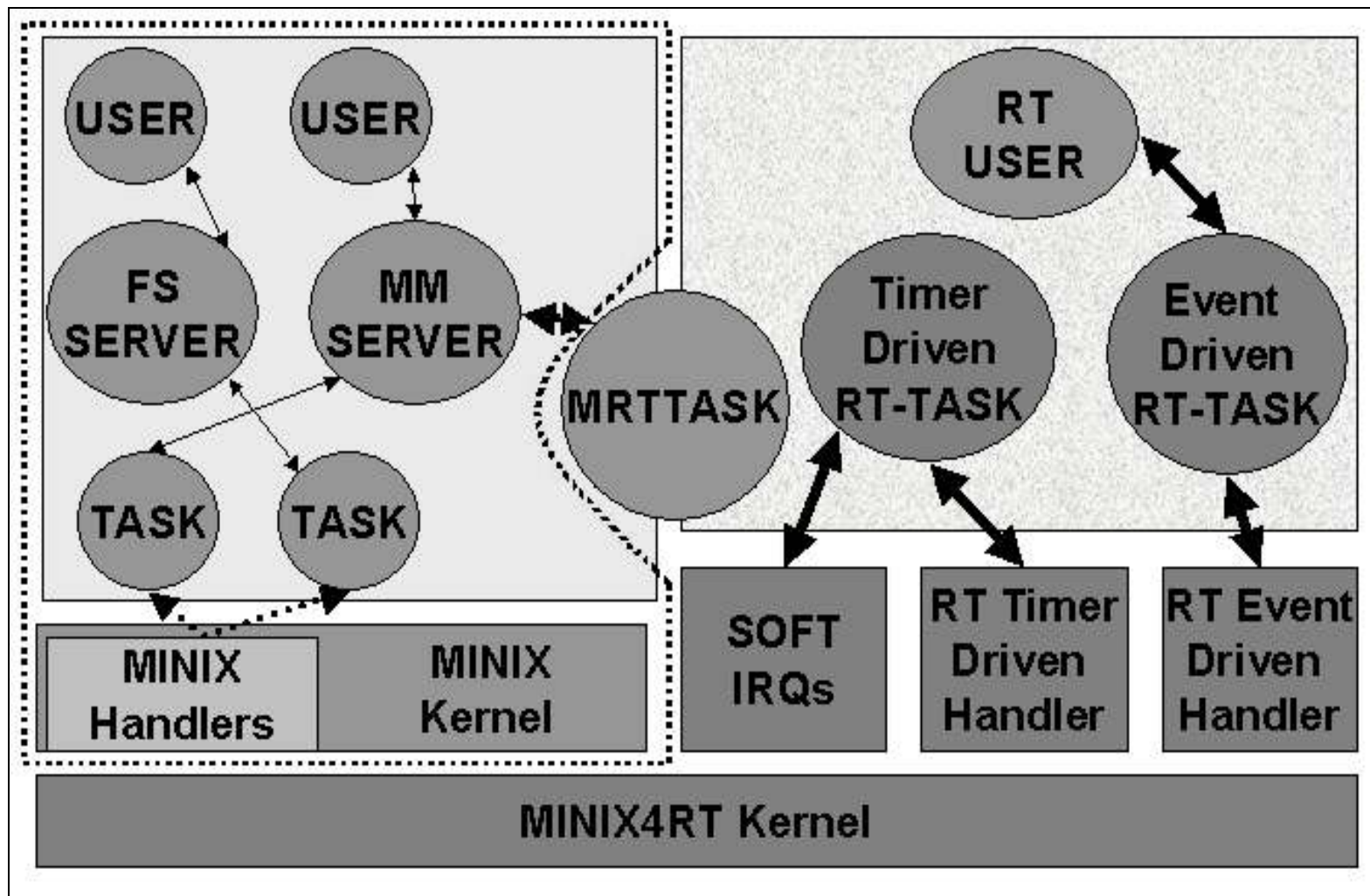

Figure 2.2: MINIX4RT Architecture.

It can be consider a RTOS to comprise two distinct classes of executable entities [19]:

1. Processes

2. Interrupt Handlers

Therefore, there is a need of two types of schedulers, one for processes and other for interrupt handlers. MINIX4RT effectively puts in place a new process scheduler that treats the MINIX kernel as the lowest priority process executing under the RT-kernel, and an interrupt handler scheduler to control the order of execution of interrupt handlers.

As NRT-interrupt handlers could block RT-processes or RT-interrupt handlers, the RT-kernel installs an interrupt dispatcher that executes the handler only if its priority is greater than the priority of the running RT-process or RT-handler. But, in spite of this fact that the handler could not be 
executed, the interrupt is accepted and a RT-kernel code is executed consuming processor time that produce an interference to the running RT-process or RT-handler.

Under that design, MINIX only executes when there are no RT-process to run, and the RTkernel is inactive. Thus, a MINIX process can never disable hardware interrupts or prevents itself from being preempted, yielding all resources to a RT-process. MINIX kernel may be preempted by a RT-process even during a System Call, so no MINIX routine can be safely called from a RT-process.

To carry out with the functionalities described in the previous paragraph, the following issues must be solved:

- Interrupts must be captured by the RT-kernel.

- RT-scheduler and RT-services must be implemented.

- RT-applications need an interface layer to interact with the RT-kernel.

- RT-applications may need transfer data and synchronize with NRTapplications.

- Full process and interrupt handler preemptability is needed.

\subsection{Interrupt Handling}

As RTLinux does, MINIX4RT uses the Virtual Machine (VM) concept limited to interrupt emulation or virtualization. Its microkernel is underneath of MINIX and it runs NRT-processes only when there are not any RT-process ready to run.

Since interrupts can come at any time, the kernel might be handling one of them while another one (of a different type) occurs. This should be allowed as much as possible since it keeps the $\mathrm{I} / \mathrm{O}$ devices busy. As a result, the interrupt handlers must be coded to run in a nested way.

When each interrupt handler terminates, the kernel must be able to resume execution of the interrupted process or switch to another process if the interrupt signal has caused a rescheduling activity or executes another lower priority interrupt handler.

Although the kernel may accept a new interrupt signal while handling a previous one, some critical regions exist inside the kernel code where interrupts must be disabled. Such critical regions 
must be limited as much as possible since, the kernel, and in particular the interrupt handlers, should run most of the time with the interrupts enabled.

MINIX4RT avoids disabling interrupts for extended periods to improve the system response time. In spite of that, the RT-kernel disables interrupts (by intervals as short as possible) to protect data structures that are also accessed by interrupt handlers avoiding race condition. The coarse time granularity among disabling and enabling interrupts could inflict unpredictable interrupt dispatch latency.

RT-interrupt handlers can easily be replaced with NRT-handlers without recompiling the kernel. This feature is especially useful in certain debugging situations.

MINIX4RT operates in two processing modes, that will be explained in Chapter 3:

- Non Real-Time Mode: In this mode, only standard MINIX interrupt handlers and NRT-processes are executed and a limited number of RTSystem Calls are allowed (i.e. a System Call that enables switching to RealTime Mode). The RT-kernel functions are disabled.

- Real-Time Mode: In this mode, the system is controlled by the RTkernel and all kind of interrupt handlers and processes can be executed. When an interrupt occurs, the RT-handler is invoked for a RT-defined interrupt, otherwise its NRT-handler is called.

The system starts in Non Real-Time Mode. To start the Real-Time Mode a NRT-process must invoke the mrt_RTstart() System Call.

\subsubsection{Interrupt Handling Virtualization}

The MINIX kernel disable interrupts for synchronization when it enters into critical sections avoiding that MINIX could be preempted when a RT-interrupt occurs.

MINIX4RT modifies some functions (and emulate its original behaviour) to avoid that the MINIX kernel could disable interrupts and could not be preempted by the RTkernel. The trick is quite simple because MINIX uses the following functions for interrupt handling:

- lock(): Disables CPU maskable interrupts (CLI for Intel x86).

- unlock(): Enables CPU maskable interrupts (STI for Intel x86). 
- put_irq_handler(): Registers an interrupt handler.

- disable_irq(): Disables a Programmable Interrupt Controller (PIC) Interrupt ReQuest (IRQ) line specified as a parameter.

- enable_irq(): Enables a PIC IRQ line specified as a parameter.

The exposed operation is guaranteed only is all device drivers and interrupt handler use those functions to handle interrupts without using assembler instructions or own code.

\subsubsection{Hardware Interrupts Emulation}

The RT-kernel installs a layer of emulation software between the MINIX kernel and the interrupt controller hardware. On RT-Mode, the emulator catches all hardware interrupts and redirects them to either standard MINIX handlers or to RT-kernel handlers. The RTkernel provides a framework onto which MINIX4RT is mounted with the ability to fully preempt MINIX.

Whenever a NRT-interrupt happens during the execution of a higher priority RTprocess, a bit in a bitmap is set by the $M R T_{-} I R Q_{-}$dispatch() function as it will be explained in Section 2.3.7. On returns of interrupts or on returns from System Calls the function $M R T \_f l u s h \_$int() is called. This function is devoted to execute all pending interrupt handlers, but if MINIX has disabled interrupts using the emulated lock() function or the PIC IRQ line for this interrupt has been disabled with the emulated disable_irq(), the NRT-handler will not be executed. Later, when the MINIX kernel (virtually) re-enables interrupts, using the emulated unlock() function, all pending interrupts are executed.

A drawback of this approach is that the MINIX kernel suffers a slight performance loss when MINIX4RT VM is added due to processing time consumed by:

- The redirection of interrupt handlers to a common interrupt dispatcher.

- The interrupt mask/unmask functions.

- The search of pending interrupts in the interrupt descriptor queues (explained in Section 2.3.12)

- The deferred execution of interrupt handlers. 
- The status and statistical information gathering as part of interrupt handling.

In consideration of both strengths and weaknesses, this strategy has shown itself to be flexible because it removes none of the capability of standard MINIX, yet it provides guaranteed scheduling and response time for critical processes.

The changes to standard MINIX are minimal with the VM approach. This low level of intrusion on the standard MINIX kernel improves the code maintainability to keep the RT-related code up-to-date with the latest release of the MINIX kernel.

\subsubsection{User and Kernel Stacks}

The stack is a LIFO list. Stacks are very useful for passing parameters between subprograms and for storage of variables or identifiers for recursive programs and languages with scope-limited variables, such as in "C". Stacks are ideal data structures for an OS process manager to track the status of processes in various states. In MINIX (and MINIX4RT) each process has two stacks:

- User-Mode stack: In User-Mode processing, only this stack can be used.

- Kernel-Mode stack: When entering the Kernel-Mode processing, the system switches to this stack.

On interrupts and system calls, the User-Mode stack is changed to the Kernel-Mode stack. If new interrupts occur during the service of other interrupts (nested interrupts), the stack remains in Kernel-Mode.

The variable $k_{-}$reenter counts the level of reentrancy in the kernel:

- $\quad k$ reenter $=(-1)$ : When the system is in User-Mode.

- $\quad k$ reenter $=0$ : When one kernel control path is running. That can be a system call, an exception/fault handler or an interrupt service routine.

- $\quad k_{\text {rreenter }}>0$ : When more than one kernel control path is running. This occurs on nested hardware interrupts. 
To monitor the Kernel-Mode stack use, each interrupt descriptor (described in Section 2.3.6) has a field named reenter that keeps the maximum kernel reentrancy level ( $k \_$reenter) for each IRQ. It helps to size the Kernel-Mode stack for specific uses.

As it is expected that a RTOS will receive much more interrupts than a time-sharing OS, by default, the RT-kernel stack doubles in size MINIX's kernel stack.

\subsubsection{Interrupt Handler Types}

Not all interrupts have the same urgency. In fact, the interrupt handler itself is not a suitable place for all kind of actions. Long non-critical operations should be deferred, since while an interrupt handler is running, the signals on the corresponding IRQ line are ignored.

MINIX4RT defines the following kinds of hardware interrupt handlers:

- Non RT-handler: When the system is in NRT-mode, only NRThandlers are executed. When the system is in RT-Mode, the NRT-handler is executed only if there are not any running RT-process or RT-handler; otherwise it is marked as triggered for later processing. The execution priority of NRT-handler is MRT_PRILOWEST.

- RT Event-Driven (ED) handler: When the system is in RT-Mode the RT ED-handler is executed only if its priority is greater than the priority of the interrupted RT-process or RT-handler, otherwise it is is marked as triggered for later processing.

- RT Timer-Driven (TD) handler: This type of handlers does not execute when the device interrupt occurs. They are executed on Timer Interrupts defined by a period. On each device interrupt, the handler is marked as triggered and it will be processed once it reaches its period following a Timer Interrupt.

The Timer interrupt handling differs from other IRQs; some actions are executed on interrupt time, but other are delayed to handle time related software facilities named Virtual Timers (VT) (explained in Chapter 4). 


\subsubsection{Interrupt Service Routines}

At startup, the RT-kernel initializes the IDT (Interrupt Descriptor Table) pointing each entries of master PIC hardware interrupts to a code generated by the macro hwint_master(irq). The entries for the slave PIC hardware interrupts are filled with the address of a code generated by the macro hwint_slave(irq).

In RT-Mode, all interrupt service routines perform the same basic actions:

- Save the registers contents in the Kernel-Mode stack.

- Increase the kernel variable $k$ _reenter (initialized in -1).

- If $k$ reenter $=0$, the state of the User-Mode process is saved, otherwise the system is already in Kernel-Mode.

- Send an acknowledgment to the PIC that is servicing the IRQ line, thus allowing it to issue further interrupts.

- Execute the interrupt handler dispatcher $M R T \_I R Q \_$dispatch().

- Terminate by jumping to the restart label if the $k_{-}$reenter $=0$ or to restart 1 label for $k$ _reenter $>0$. More details in Section 2.3.11.

\subsubsection{Interrupt Descriptor Data Structure}

This section explains the data structures that supports interrupt handling and how they are laid out in various descriptors used to store information on the state, statistics and behavior of interrupt handlers.

The RT-kernel has its own interrupt descriptor table (other than INTEL's IDT) named $M R T \_s i . i r q t a b[]$. It is an array of $M R T \_i r q d \_t$ data structures that has one descriptor for each hardware and software interrupt (explained in Section 2.3.10.).

The $M R T \_i r q d \_t$ data structure has the following functional fields (see Appendix D):

- nrthandler: A pointer to a function that is the NRT-handler.

- rthandler: A pointer to a function that is the RT-handler. 
- period: The processing period of a TD-interrupt handler in RT-ticks units.

- task: The RT-handler will not execute, instead a MT_INTERRUPT message will be sent to the speciried task when an interrupt occurs.

- watchdog: The RT-PID (RT process ID defined by mrtpid_t data type) of a RT-process that will take corrective actions against RT-handler missed deadlines. When a handler does not complete it's work before it's deadline, the RT-kernel sends a MT_DEADLINE message to the specified watchdog process.

- priority: Specifies the handler priority.

- irqtype: Specifies type of handler. It is a logical-OR of the following attributes:

- MRT_RTIRQ: Real-Time handler (otherwise it will be NRT-IRQ).

- MRT_TDIRQ: Timer-Driven handler (otherwise it will be ED-IRQ).

- MRT_SOFTIRQ: Software interrupt handler, explained in Section 2.3.10. (Otherwise it will be a hardware interrupt handler).

The MRT_irqd_t data structure has the following fields for kernel internal use:

- irq: The IRQ number.

- harmonic: It is the harmonic frequency of the MINIX Timer interrupt frequency (stored in a system variable named $M R T$ sv.harmonic) when RTprocessing Mode starts. It is only used for Timer-Driven Interrupt descriptors to convert the period of a TD-interrupt handler when the user has changed the Timer interrupt frequency. More details in Chapter 4.

- pvt: A pointer to an assigned VT for TD-interrupt descriptors.

- flags: Some interrupt descriptor status and configuration flags that determines its behavior.

- shower: A counter for TD-interrupts in the last period (explained in Section 2.3.20). 
- latency: The estimated interrupt handler latency in Timer Hz.

- before: An auxiliary field that stores the Timer-2 latch counter of the Programmable Interrupt Timer (PIT) in Hz on MRT_IRQ_dispatch() entry.

- next: A pointer to the next interrupt descriptor in the queue.

- prev: A pointer to the previous interrupt descriptor in the queue.

- name: The name of the handler.

The $M R T$ irqd_t $t$ data structure has the following statistical use fields:

- count: An interrupt counter for statistics.

- $\quad m d l$ : A missed deadlines counter.

- maxshower: Stores the maximum value of shower.

- timestamp: The last interrupt timestamp.

- maxlat: The maximum value of latency.

- reenter: Stores the maximum value of $k_{-}$reenter. (Explained in Section 2.3.3).

\subsubsection{Interrupt Handler Dispatching}

MINIX4RT VM sets all Hardware Interrupt Service Routines (ISR) labeled as HWINTXx to call an interrupt dispatcher function named MRT_IRQ_dispatch(). This function base its decisions to process interrupts based on the interrupt descriptor table MRT_si.irqtab[](see Figure 2.3).

A RT-kernel variable called MRT_sv.prtylvl stores the current system priority level of execution. This variable is set to the current process priority or to the running interrupt handler's priority. It is used to reduce the unbounded priority inversion problem deciding on the execution of an interrupt handler or to defer it.

If MINIX4RT is running in NRT-mode, the standard interrupt handlers (irq_table[irq] (irq) in Figure 2.3) are executed without any interception, deferring and statistics gathering. 
In RT-Mode, the function $M R T \_I R Q \_$dispatch() performs the following actions (see Figure 2.3 and Figure 2.4):

- Some statistics-related variables are updated as:

- The system wide interrupt counter named MRT_sv.counter.interrupts.

- The descriptor interrupt counter named MRT_si.irqtab[irq].count.

- The kernel reentrancy level named MRT_si.irqtab[irq].reenter.

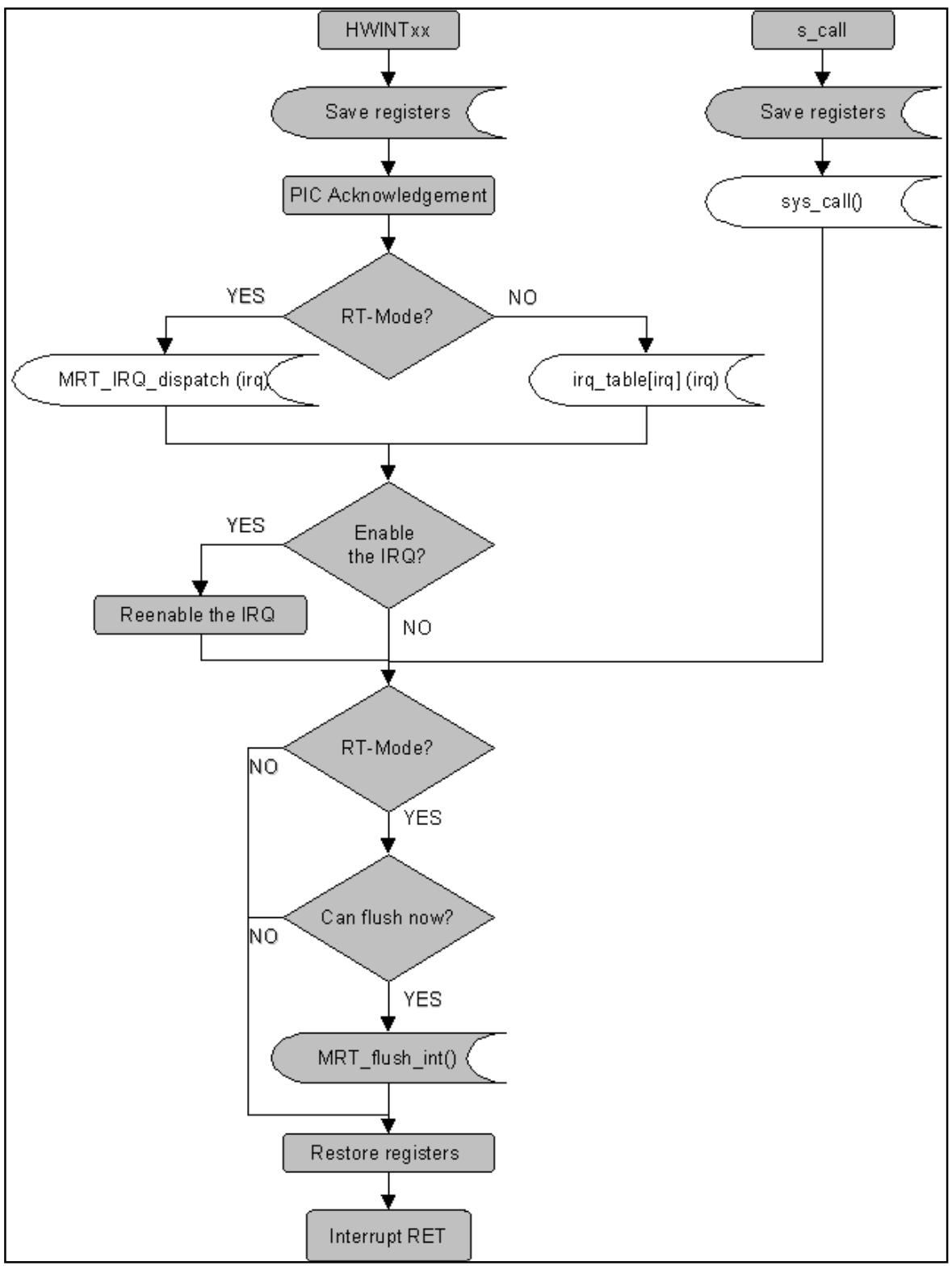

Figure 2.3: Hardware Interrupt Handling. 
- If the bit MRT_LATENCY for latency computation has been set in the descriptor, the timer- 2 of the PIT is read before running the handler and its value is stored in the before descriptor field.

- If a Timer interrupt has occurred:

- The system tick counter MRT_sv.counter.ticks is increased.

- The interrupt descriptor timestamp field is set.

- The Timer interrupt descriptor is marked for deferred processing only if it has some additional work to do controlled by Virtual Timers (more in Chapter 4).

- Returns from Interrupt and reenable the IRQ0 of the PIC.

- For interrupts other than the Timer, the interrupt descriptor timestamp field MRT_si.irqtab[irq].timestamp is set to MRT_sv.counter.ticks.

- If an Event-Driven (ED) interrupt has occurred with its priority greater than or equal to $M R T \_s v . p r t y l v l$, the handler is called (Note: higher priority means a lower value in the priority field). The handler can signal the kernel to run the RT-scheduler on exit setting a bit (MRT_SCHEDULE) in the kernel variable $M R T \_s v$.flags.

- If an ED-interrupt has occurred with its priority lower than $M R T \_s v . p r t y l v l$, the descriptor is marked for deferred processing.

- If a NRT-interrupt has occurred with its priority greater or equal than $M R T \_s v . p r t y l v l$, the handler is called and then exits.

- If a NRT-interrupt has occurred with its priority lower than MRT_sv.prtylvl, its descriptor is marked for deferred processing.

- If a TD-interrupt has occurred, the bit MRT_TDTRIGGER in flag field of the interrupt descriptor is set. This bit signals the interrupt associated VT that the TD-handler must be executed on the next period. The operation of TD-interrupts using VTs is explained in Chapter 4. 


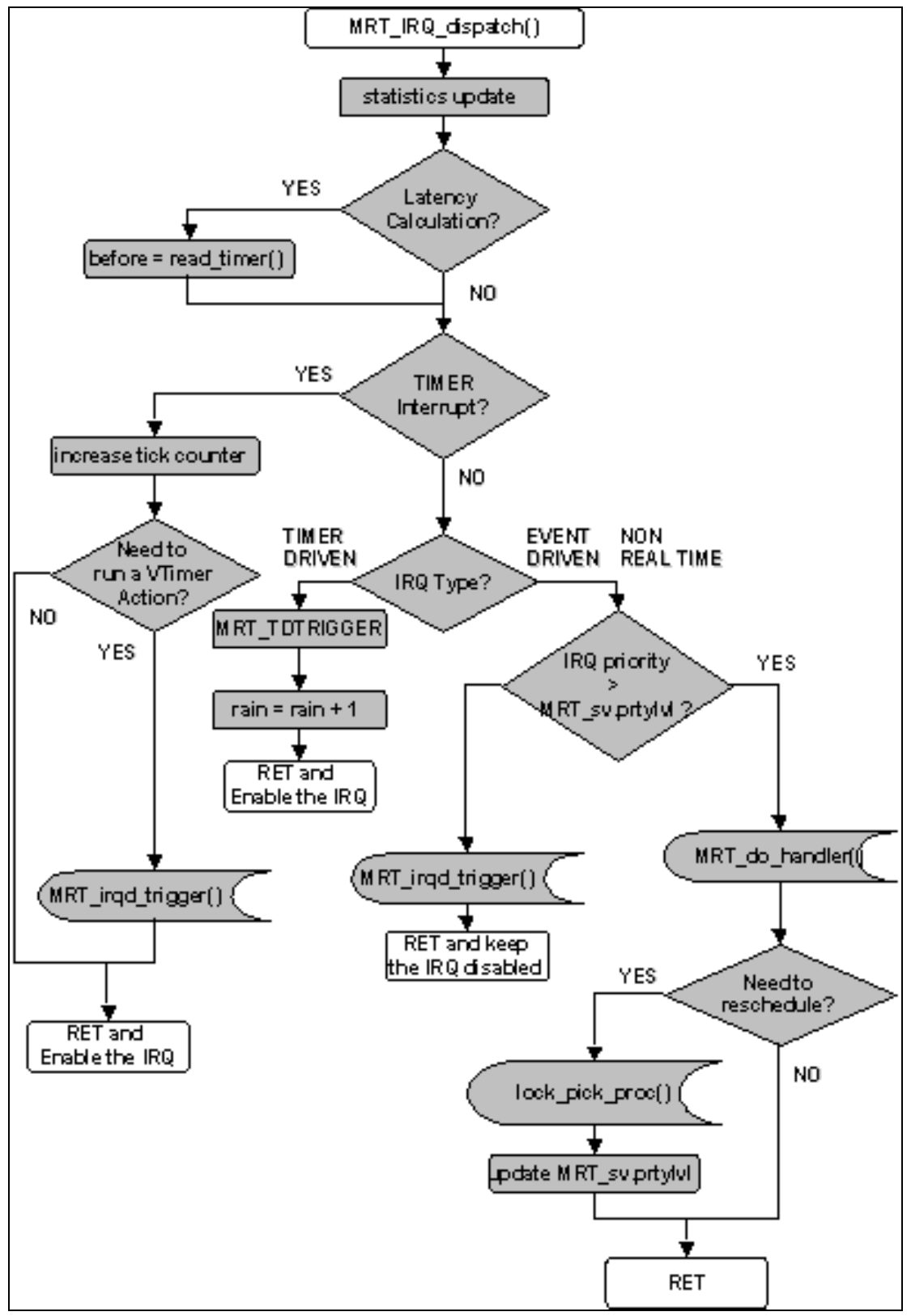

Figure 2.4: Interrupt Handler Dispatching.

\subsubsection{Interrupt Handler's Priority}

The PIC treats interrupts according to their priority level that are directly tied to the IRQ number. Higher-priority IRQs may improve the performance of the devices that use them.

The standard PC hardware has assigned priorities for standard interrupts related to IRQ number as shown in Figure 2.5. A lower IRQ number implies higher priority. Newer Advanced Programmable Interrupt Controllers (APICs) allow programmers to change the 
priority of each IRQ line. The RT-kernel attends IRQs based on the priority field of their descriptors.

If a lower priority interrupt occurs during the execution of a running process or handler, its interrupt descriptor is marked as triggered and its processing is delayed. Later, after a context switch, a System Call or a Return from Interrupt, the RT-kernel calls $M R T \_$flush_int() function that scans the queues for interrupt descriptors that has been triggered and runs their handlers.

$M R T \_$flush_int() alternatively calls $M R T$ _irqd_flush() that scans for triggered interrupt descriptors and MRT_vtimer_flush() that runs actions of expired VTs (see Section 4.8.3) in priority order.

The following pending interrupt handlers are not executed by MRT_irqd_flush():

- NRT-interrupts once MINIX has (virtually) disabled processor interrupts using the emulated $\operatorname{lock}()$ function.

- All pending interrupt handlers with lower priority than the current system priority level of execution $M R T \_s v \cdot p r t y l v l$.

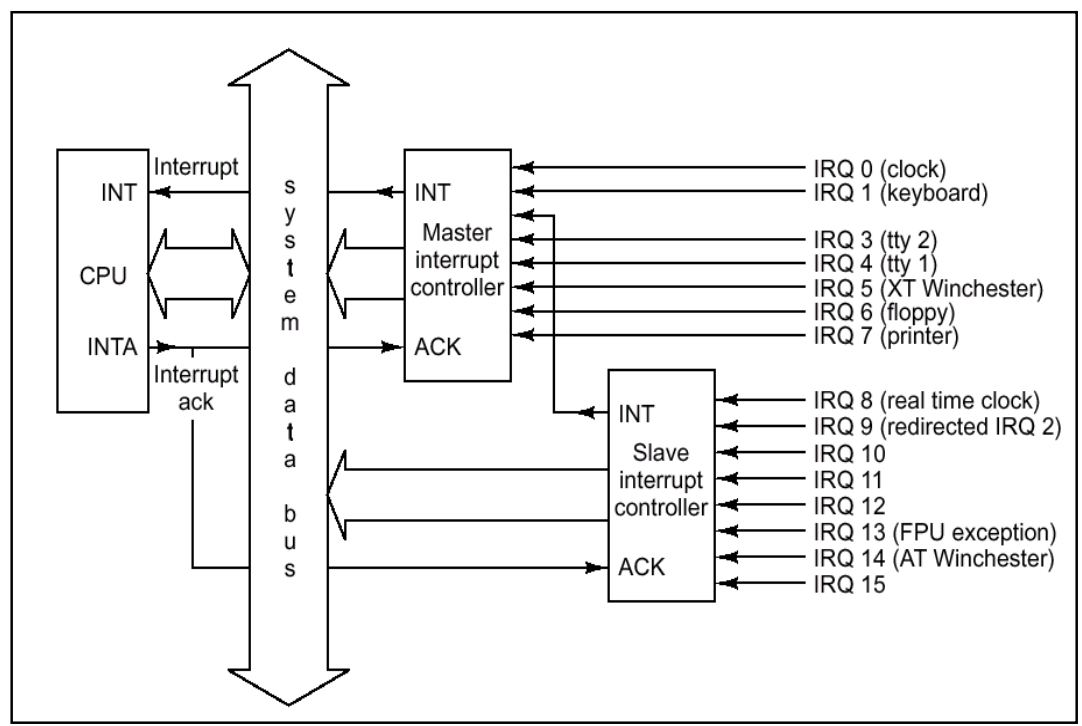

Figure 2.5: IRQ Priorities (from [2]).

Each RT-handler must notify the RT-kernel setting the bit MRT_SCHEDULE in $M R T \_s v . f l a g s$ if it needs to run the RT-scheduler on exit 


\subsubsection{Real-Time Input/Output Tasks}

In MINIX, as in other OSs, each kind of input/output device has a related device driver. MINIX device drivers have two components:

- An Interrupt Service Routine (ISR) that catchs device interrupts.

- An I/O Task for delayed interrupt processing and to accept requests from server processes.

A was explained in Chapter 1, Task is a special kind of MINIX process that has its own state and stack, but shares the kernel address space with the kernel and other Tasks (explained in Section 2.1). Server processes requests services to Tasks using standard message transfers. When a Hardware interrupt occurs, the kernel signals the I/O Task sending it a message, but this fact usually involves a context switch because the Task preempts the running process when receives the message causing a performance penalty.

MINIX4RT allows device driver writers to adopt the use of RT-Tasks for their design. As any RT-process, a RT-Task has an assigned priority and any RT-processes could request services to it using RT-Interprocess Communications (explained in Chapter 5).

The interrupt descriptor associated with that RT-Task can be a defined as a TDinterrupt or an ED-interrupt setting or clearing the MRT_TDINT bit of the irqtype field of the interrupt descriptor.

\subsubsection{Software Interrupts}

One of the main problems with interrupt handling is how to perform longish tasks within a handler. Often a substantial amount of work must be done in response to a device interrupt, but interrupt handlers need to finish up quickly and not keep interrupts blocked for long. These two needs (work and speed) conflict with each other, leaving the driver writer in a bit of a bind. Therefore it is desirable that the interrupt handlers could delay the execution of some tasks so that they do not block the system for too long.

As it is explained above, MINIX uses a two stages interrupt management where an interrupt handler partially process the interrupt and then sends a message to an I/O Task to resume the interrupt processing. This approach implies at least a context switch to restore the state of the task. 
Linux kernel resolves this problem by splitting the interrupt handler into two halves. The so-called top half is the routine that actually responds to the interrupt. The bottom half is a routine that is scheduled by the top half to be executed later, at a safer time.

Programmers have at least three approaches to design Device Drivers:

A. Complete interrupt processing into the handler.

B. Two stages interrupt management like MINIX using RT-Tasks and message transfers.

C. Two stages interrupt management using Software interrupts as Linux does with bottom halves.

Triggered Software interrupts are kernel routines that are invoked by $M R T \_$flush_int() as it do with pending hardware interrupts.

Software interrupt descriptors have the same data structure $M R T$ irqd_t than Hardware interrupts descriptors, therefore they have priority, counters, timestamps and other fields.

The motivation for introducing software interrupt is to allow a limited number of functions related to interrupt handling to be executed in a deferred manner. This approach increases the system responsiveness because some work is executed out of interrupt time. Moreover, the processing overhead is lower than using the I/O Task model because it avoids the context switch among the interrupted process and the I/O Task. Software interrupts can be used as kernel threads triggered by time using Virtual Timers.

\subsubsection{Returning from System Calls and Interrupts Service Routines}

The main purpose of the termination phase of interrupt/exception handlers and System Calls is to execute the highest priority process, but several issues must be considered before doing it. The system needs to execute:

- Pending Event Driven Interrupt handlers.

- Pending Software interrupt handlers.

- Virtual Timer Actions (explained in Chapter 4). 
The RT-kernel will execute only those pending issues that have greater priorities (lower priority field) than the MRT_sv.prtylvl.

Before returning the CPU to User-level Mode those interrupt handlers that has been deferred must be executed. The RT-kernel scans the interrupt descriptor queue for hardware and software pending interrupt handlers and invokes only those handlers with priorities greater than the current process priority. The kernel function that accomplishes with these issues is MRT_flush_int() (see Figure 2.3).

\subsubsection{Flushing Deferred Interrupts}

MINIX4RT uses a system of interrupt descriptors queues and a bitmap named $M R T$ si.iQ.bitmap to process deferred interrupts. A bit set in the i-th position of the bitmap implies that at least one descriptor in the $\mathrm{i}$-th interrupt descriptor queue has been triggered for deferred processing (see Figure 2.6).

An interrupt descriptor is inserted into the queue using the kernel function $M R T \_i r q Q \_i n s($ (explained in Section 2.3.15), and it remains in the queue even once the interrupt has been serviced. Those enqueued interrupt descriptors that need service have a bit (named MRT_TRIGGERED) of the flag field set.

Some test performed by the author during the implementation, proved that inserting a descriptor when the interrupt occurs and removing it once the interrupt has been serviced, has less perfomance that keep the descriptors enqueued. Moreover, the processing time required with the CPU interrupts disabled is reduced improving kernel preemptability.

$M R T$ flush_int() scans for bits set in two bitmaps, the MRT_si.iQ.bitmap for deferred interrupts and the MRT_st.tQ.bitmap for expired VTs actions to execute. The scan stops when the priority of the represented by the bit position is lower than MRT_sv.prtylvl (see Figure 2.7).

For each bit set in MRT_st.tQ.bitmap, MRT_flush_int() calls MRT_vtimer_flush() that executes expired VTs actions. This operation is explainded in Section 4.8.2.

For each bit set in MRT_si.iQ.bitmap, MRT_flush_int() calls RMT_irqd_flush() that scans the interrupt descriptor queue for hardware and software interrupt handlers triggered to run. A handler is executed only if its descriptor status flags has the MRT_TRIGGERED 
bit set and has not been disabled (MRT_DISABLED) by software. Once all triggered interrupt descriptors in the same queue has been serviced, the bit in MRT_si.iQ.bitmap is cleared.

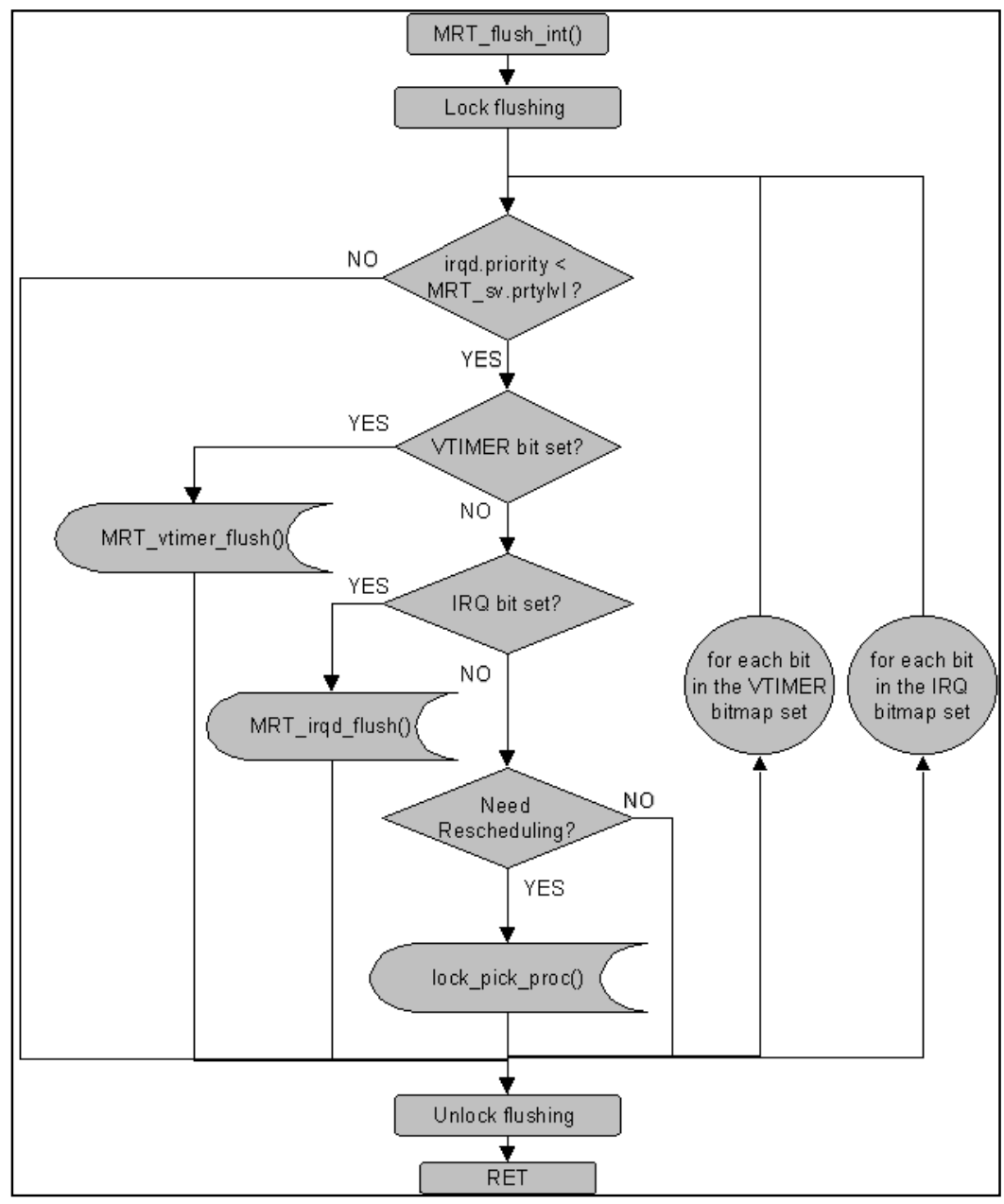

Figure 2.6: Interrupt Bitmap and Interrupt Queues.

If one of the handlers has set the MRT_SCHEDULE bit of the system variable $M R T \_s v . f l a g s$, the scheduler (lock_pick_proco function) is invoked.

Each interrupt queue descriptor has two counter fields:

- inQ: Records the number of descriptors enqueued. It is increased by the $M R T \_i r q Q \_i n s()$ and decreased by $M R T \_i r q Q \_r m v()$ kernel functions.

- pending: Records the number of triggered interrupts in the queue. It is increased when some descriptor is triggered by the MRT_irqd_trigger ( kernel function and is decreased by the MRT_irqd_serviced(). 


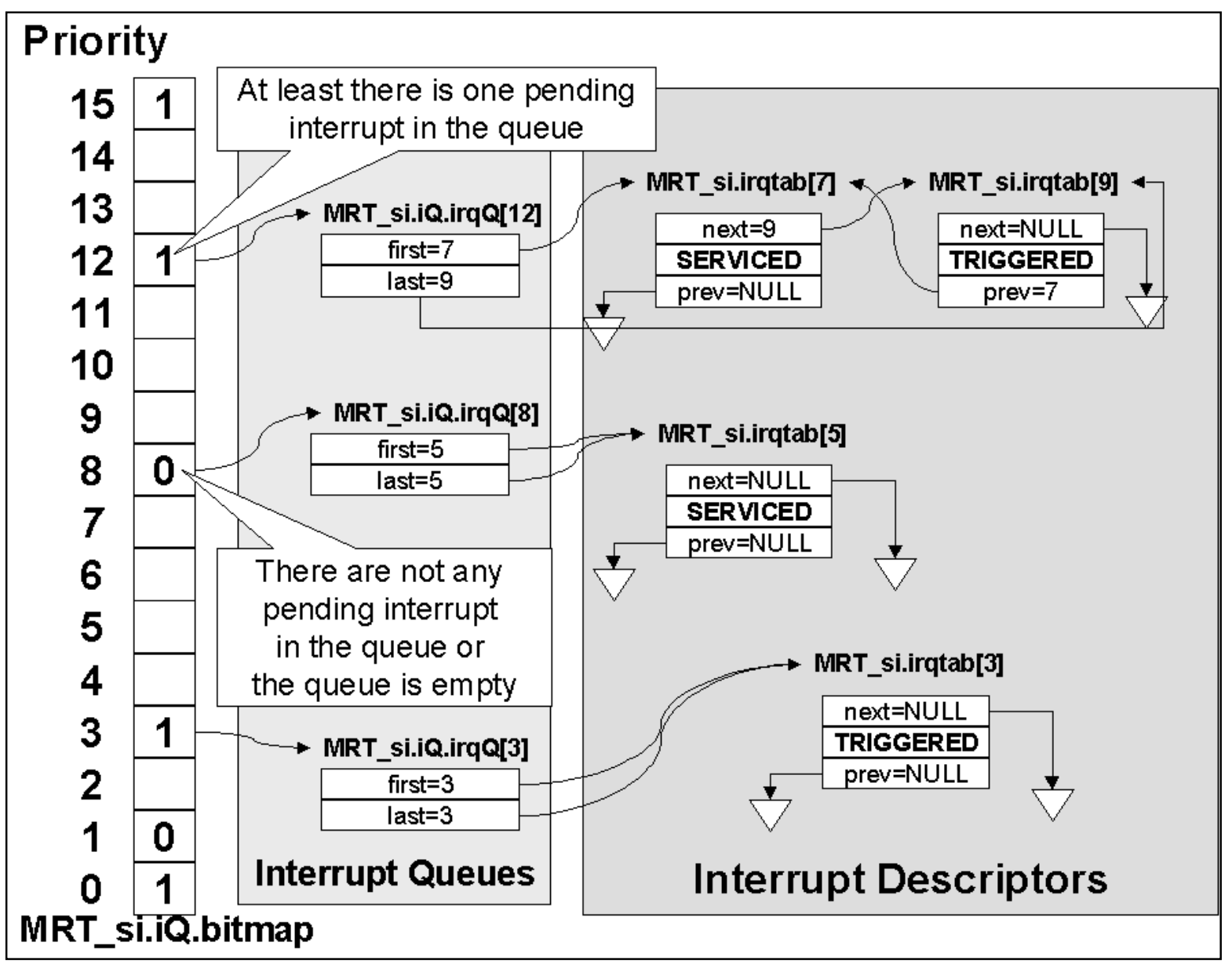

Figure 2.7: Flushing Interrupts and Virtual Timers.

Only that queues with (in $Q>1)$ will be scanned to find the triggered descriptors. The scanning will stop when (pending $=0$ ).

As during the execution $M R T$ flush_int(), new interrupts can occur, a lock bit (MRT_FLUSHLCK) in the kernel variable MRT_sv.flags is set to avoid that MRT flush_int) could be called again returning from a nested hardware interrupt. If the nested interrupt has greater priority than the interrupted handler, another bit named MRT_NEWINT in $M R T \_s v$.flags is set to notify $M R T \_f l u s h \_i n t()$ that it needs to restart the scan again from the highest priority interrupt queue to find the new triggered interrupt descriptor.

\subsubsection{Executing Interrupt Handlers}

Before running interrupt handlers, the RT-kernel must (see Fig 2.8):

- Save the current system priority level of execution MRT_sv.prtylvl.

- Set MRT_sv.prtylvl to the Interrupt handler's priority. 
- Restore CPU flags to its previous state.

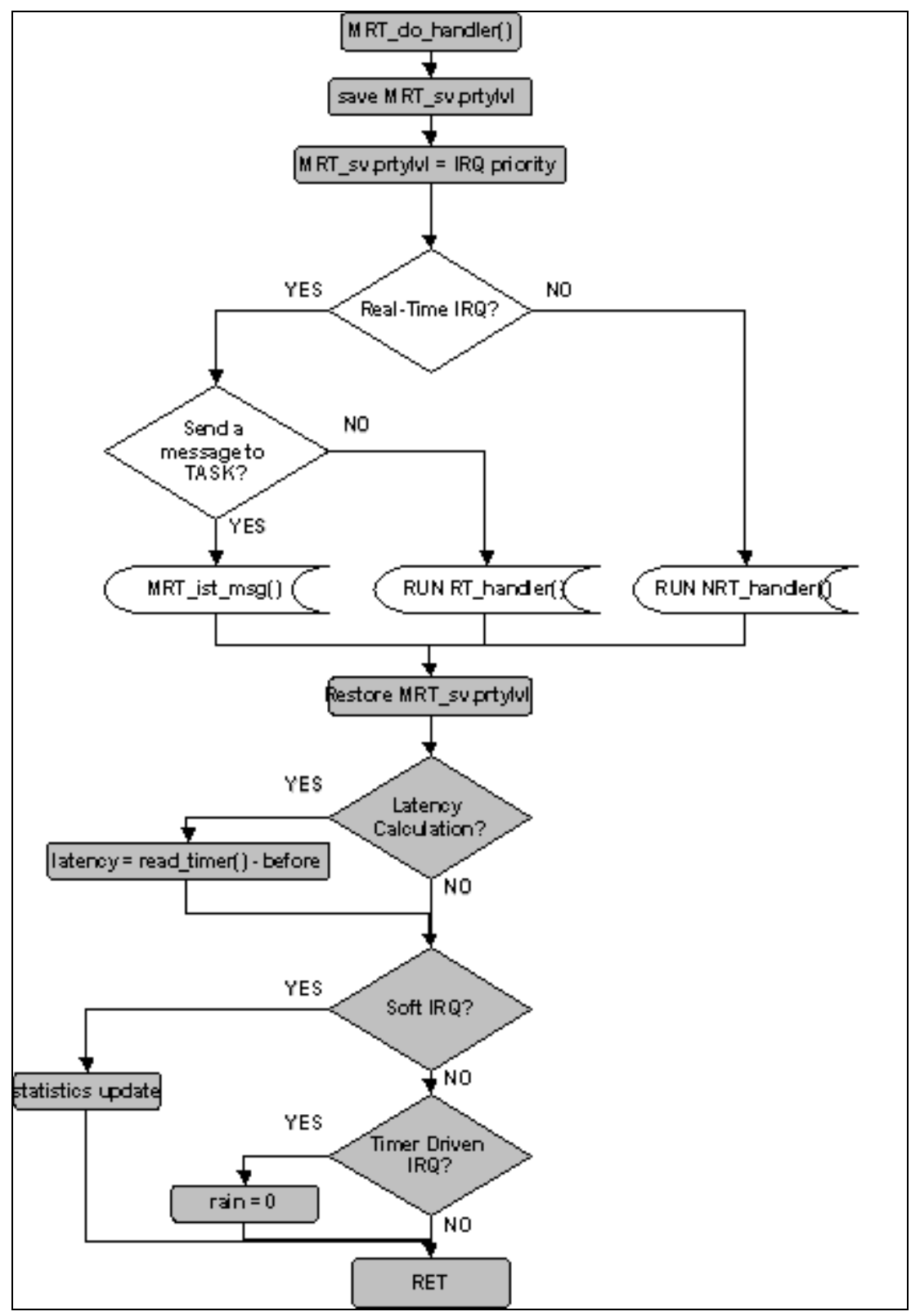

Figure 2.8: Running Interrupt Handlers.

If the interrupt descriptor flag field has the bit $M R T_{-} I S T M S G$ set, instead of executing a handler, the RT-kernel sends a MT_INTERRUPT message to the RT-Task specified in the task field of the interrupt descriptor.

Once the handler exits the CPU flags are saved in the stack and the interrupts are disabled and an estimation of the handler processing time could be computed (see Section 2.3.16). 
The value returned by a hardware interrupt handler notifies the kernel if the PIC IRQ line must be enabled or not.

\subsubsection{Interrupt Descriptor Timestamp Field}

MINIX4RT includes a timestamp field in the interrupt descriptor data structure $M R T \_i r q d \_t$. For hardware interrupts this field is set by $M R T \_I R Q \_$dispatch() to the value stored in MRT_sv.counter.ticks. For software interrupts timestamp field is filled after the handler was executed into the MRT_do_handler() function.

The units of the timestamp field are RT-ticks since the last execution of the mrt_RTstart() or mrt_restart() System Calls.

\subsubsection{Kernel Functions for Interrupt Handling and Synchronization}

Interrupt disabling is one of the key mechanisms used to ensure that a sequence of kernel statements is treated as a critical section. It allows a kernel control path to continue executing even when hardware devices issue IRQ signals, thus providing an effective way to protect data structures that are also accessed by interrupt handlers [15].

On Intel x86 architecture, when the kernel enters a critical section, it clears the $I F$ flag of the EFLAGS register to disable interrupts. But, at the end of the critical section, often the kernel can not simply set the flag again. As interrupts can execute in nested fashion, the kernel does not necessarily know what the $I F$ flag was before the current control path executed. In these cases, the control path must save the old setting of the flag and restore that setting at the end [15].

The RT-microkernel has the following functions to control interrupts:

- MRT_lock(): Disables interrupts at the CPU (CLI assembler instruction on INTEL).

- MRT_unlock(): Enables interrupts at the CPU (STI assembler instruction on INTEL).

- MRT_saveFlock(\&CPUflags): Saves the current CPU Flag Register into the stack and then clears the $I F$ (interrupt flag) using the $C L I$ assembler instruction to disable interrupts. 
- MRT_restoreF(\&CPUflags): Restores the CPU EFLAGS Register from the stack.

Each IRQ line can be selectively disabled. Thus, the PIC can be programmed to disable IRQ lines. That is, the PIC can be told to stop issuing interrupts that refer to a given IRQ line, or to enable them. Disabled interrupts are not lost; the PIC sends them to the CPU as soon as they are enabled again. This feature is used by most interrupt handlers since it allows them to process IRQs of the same type serially.

Selective enabling/disabling of IRQs is more efficient than global masking/unmasking of maskable interrupts but they are more time consuming.

The RT-microkernel has the following functions to handle IRQs at PIC level:

_ MRT_disable_irq(): Disables an IRQ line at PIC level.

- MRT_enable_irq(): Enables an IRQ line at PIC level.

- MRT_get_PIC(): Reads the PIC mask.

- MRT_set_PIC(): Sets the PIC with a specified mask.

Interrupt descriptors are used to store information on the state, statistics and behavior of interrupt handlers. The RT-kernel has the following functions to handle interrupt descriptors:

- MRT_irqd_set(): Sets all fields of an interrupt descriptor.

- MRT_irqd_free(): Removes an interrupt descriptor from its interrupt descriptor queue. It resets all fields of an interrupt descriptor. The RThandler and NRT-handler points to the spurious_irq() handler.

- MRT_softirq_set(): Assigns a new interrupt descriptor from the software interrupt descriptor pool, then sets the descriptor using MRT_set_irq().

- MRT_softirq_free(): Removes the descriptor from the interrupt descriptor queue using MRT_irqd_free() and frees the descriptor to the software interrupt descriptor pool.

- MRT_irqQ_ins(): Inserts an interrupt descriptor into an interrupt descriptor queue. 
- MRT_irqQ_rmv(): Removes an interrupt descriptor from an interrupt descriptor queue.

Two kernel macros can be used to disable/enable descriptors:

- MRT_disable_irqd(): disables an interrupt descriptor in such a way that it will not be executed by the kernel even if triggered.

- MRT_enable_irqd(): enables an interrupt descriptor to be executed by the kernel if triggered.

Those macros set/clear the bit MRT_DISABLED in the flag field of the interrupt descriptor that is checked before running the handler.

\subsubsection{Estimating Interrupt Handler Processing Time}

A vital characteristic of a RTOS is how responsive is in servicing internal and external events. These events include external hardware interrupts, internal software signals, and internal Timer interrupts. One measure of responsiveness is latency, the time between the occurrence of an event and the execution of the first instruction in the interrupt code. A second measure is jitter, the variation in the period of nominally constant-period events.

One of the most common measurements requested to RTOSs is the Interrupt Service Time[20]. It is used to measure the effectiveness of a RT-kernel at dealing with extremely high-priority interrupts or emergency interrupts. Often a peripheral must be serviced within a certain time limit after an event. For example, a packet must be read from a network port before the next on arrives.

The Interrupt Service Time $\left(\boldsymbol{T}_{\text {ist }}\right)$ is the maximum time taken to respond to an interrupt request. It includes the time it takes for the current instruction to complete, the time for the CPU to respond to the interrupt $\left(\boldsymbol{T}_{\boldsymbol{i}}\right)$ and the interrupt handler processing time $\left(\boldsymbol{T}_{\boldsymbol{i n t}}\right)$ (see Figure 2.9).

$$
T_{i s t}=T_{i l}+T_{\text {int }}
$$

Understanding the relative size of delays is important to the design of the RTsystem. Most sources of delay in a RT-kernel are due to either code execution or context 
switches. Virtually all of these delays are fixed in length and repeatable. Bounded and repeatable is the fundamental characteristic desired of a RT-kernel.

Interrupt Service Time are not fixed in length. Because an interrupt is, by definition, an asynchronous event, an Interrupt Service Time depends on the state of the machine when the interrupt occurred. This state is a function of both the hardware and the software used in the system.

MINIX4RT uses the Timer-2 of the Programmable Interrupt Timer (PIT) to estimates the interrupt handler processing time $\left(\boldsymbol{T}_{\boldsymbol{i n t}}\right)$. The PIT is programmed in SQUARE_WAVE mode and the divisor latch is set to 0 (to get a 65536 latch value) and its frequency is $1193182[\mathrm{~Hz}]$, therefore its period is $65536 / 1193182=0.0549$ [s].

At the start of $M R T \_I R Q \_$dispatch() kernel function, the value of the counter of the Timer-2 latch of the PIT is stored in the before field of the interrupt descriptor. On handler return, the counter is read again and the handler processing time could be estimated as the difference between those two values. The time units of the estimated value are PIT Hz or $(1193182)^{-1}[\mathrm{~s}]=8,38 \times 10^{-7}[\mathrm{~s}]=0.838[\mu \mathrm{s}]$.

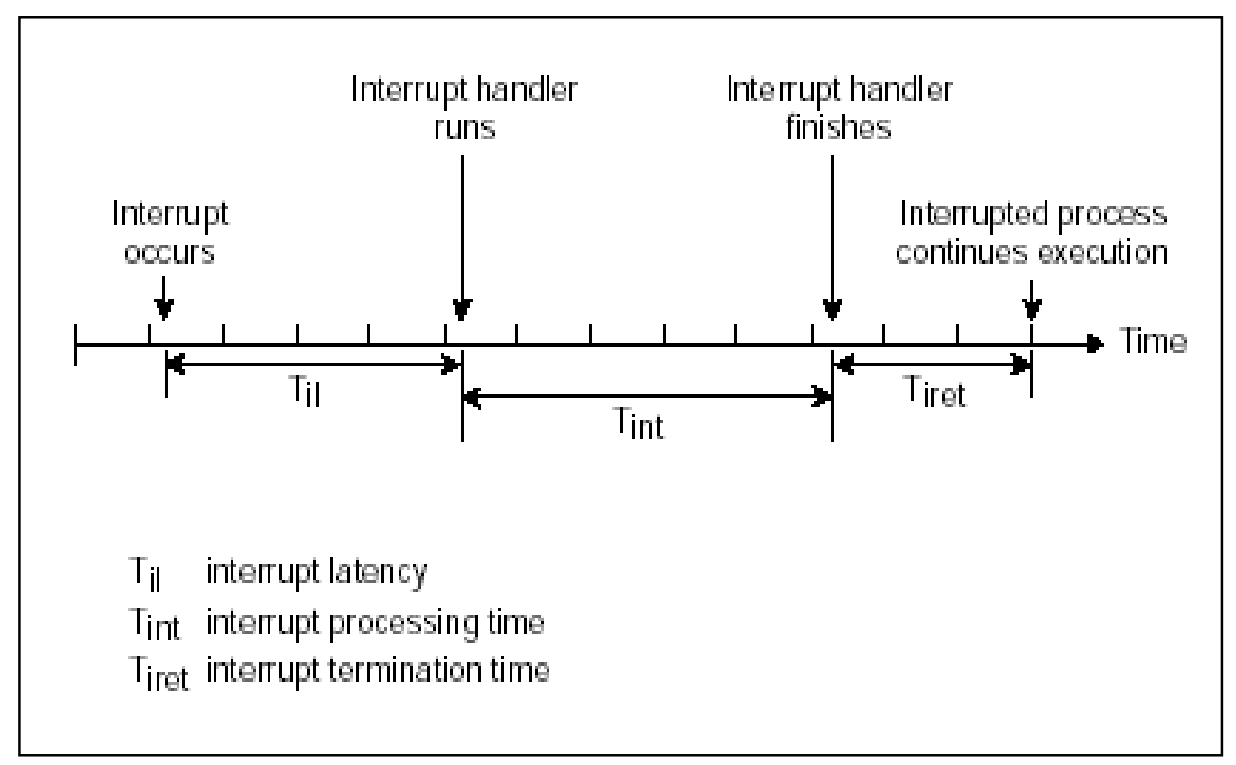

Figure 2.9: Interrupt Service Time (from [11]).

While the estimated interrupt handler processing time is not so useful as the Interrupt Service time ( $\boldsymbol{T}_{\text {ist }}$ ), it can be used to compare performance among several handler algorithms. 
The PIT Timer-0 is programmed in SQUARE_WAVE mode as Timer-2. The PIT signals the Timer Interrupt once the Timer- 0 counter reaches zero. As the Timer-0 remains decreasing the counters, during the execution of the Timer handler, the value of the Timer- 0 counter lets compute the latency of the Timer handler. The estimated value by this method represents the Interrupt Latency $\left(\boldsymbol{T}_{\boldsymbol{i l}}\right)$ of the Timer interrupt.

\subsubsection{Nested Interrupts}

As has been mentioned above, MINIX4RT supports nested interrupts. Worst-case timing considerations for unmasked interrupts must be included in the computation of the service time for all interrupts currently being processed.

The following criteria is established for nested interrupts:

- A higher or equal priority interrupt will preempt a running handler. The computation time of the higher priority handler must be added to the Service Time as a Blocking Time.

- A lower priority interrupt will not preempt a running handler. The execution of the new interrupt handler will be deferred for later processing and the function $M R T_{\_}$flush_int() will not be invoked on exit to reduce the Blocking Time. This blocking time is a priority inversion that must be added to the running handler service time. It must be consider only once because the lower priority interrupt will be disable at PIC level until the handler will re-enable it.

Once the handler exits, the RT-scheduler could be invoked and another process preempts the former as Process $B$ and Process $C$ preempts Process $A$ in Figure 2.10.

Next MINIX4RT releases will include an interrupt mask array named $M R T \_s v . m a s k[]$ used to change the PIC mask before running a handler/process to avoid that could be interrupted by a lower priority interrupt. The array items are filled by the $M R T$ irqd_set() kernel function based on the interrupt descriptor's priority. When a new interrupt descriptor is set, all items of the $M R T_{-} s v$.mask [] array are recomputed with PIC masks that consider the new descriptor's priority and the IRQ lines that must be disabled when the process/handler with this priority will run. 


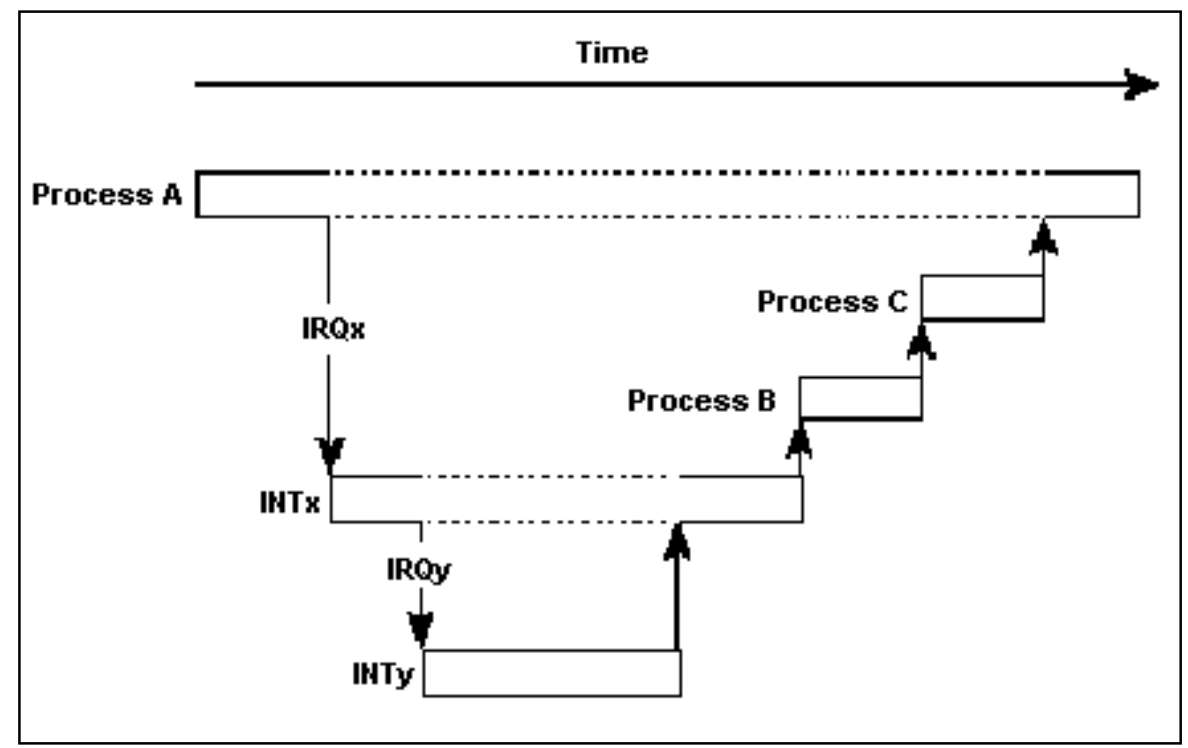

Figure 2.10: Nested Interrupts (from [11]).

\subsubsection{Real-Time Interrupt Processing}

The following categories of real-time implementations are proposed in [21]:

- Event Driven Implementation

- Integrated Interrupt Event Driven Scheduling: Is integrated in the sense that hardware interrupt priorities are matched with the software process priorities. All processes are initiated by external interrupts.

- Non-Integrated Interrupt Event Driven Scheduling: The priority of the interrupt associated with process arrival has no correspondence to the software priority of the process, and is thus non-integrated.

- Timer Driver Implementation

- Timer Driven Scheduling: A timer expires every $\boldsymbol{T}_{\boldsymbol{t} i \boldsymbol{c}}$ seconds causing a non-maskable interrupt that force a scheduling point. The scheduler moves all processes that have next scheduling points greater or equal than the current time to the ready queue.

- Timer Driven Scheduling with counter: The Timer handler decrements a counter on every timer interrupt and will only invoke the scheduler when the counter expires. The counter limits the scheduler to run only on Timer interrupts that correspond to process arrivals. 
MINIX4RT does not match strictly in any of these categories but it depends on the set of processes running on the system and the type of hardware where it runs.

MINIX4RT could be considered as an Event Driven with Non-Integrated Interrupts Scheduling and Timer Driven with counter system.

- Event Driven: On ED-interrupts the handlers are called without delay.

- Non-Integrated Interrupts: Hardware interrupt priorities could no match with process priorities.

- Timer Driven with counter: The RT-Timer interrupt handler (VT really) invokes the scheduler only when a TD-process must be scheduled or when a TD-interrupt has occured in the last timer period.

\subsubsection{Standard MINIX Non Real-Time interrupts}

In RT-mode, NRT-handler are executed only if there are not a RT-process or RThandler running when the NRT-interrupt occurs. The handler execution is delayed until $M R T$ flush_int() invokes it.

\subsubsection{Real-Time Timer-Driven Interrupts}

Some devices will raise interrupts at high rates but the interrupt processing can be delayed to be managed by a periodic process in the next schedule (Timer Driven).

Other devices do not raise interrupts, and a periodic process can be created to poll the devices checking their status and taking appropriated action.

This kind of interrupt processing lets that more than one interrupt occur in a time period without running the handler. The handler only is executed at a specified period reducing the system overload. MINIX4RT assigns a VT for the TD-interrupt descriptor.

The processing of a TD-interrupt handler has the following stages (see Figure 2.11):

- The TD-interrupt occurs and MRT_IRQ_dispatch() sets the $M R T$ TDTRIGGER bit in the flag interrupt descriptor field to signal the descriptor for later processing when its assigned VT reaches its period. The 
shower field (counts the number of TD-interrupts since the last period) is increased.

- On each Timer interrupt, $M R T \_I R Q \_$dispatch() checks for expired VTs. If any VT has expired, the Timer handler is triggered and it will run in the next call to $M R T \_$flush_int().

- MRT_flush_int() runs the Timer handler that enqueues expired VTs for later processing of their actions. More details in Chapter 4.

- MRT_flush_int() calls MRT_vtimer_flush() that runs actions of those expired VTs. The action of a VT assigned to a TD-interrupt handler is $M R T \_A C T$ IRQTRIG, that triggers the descriptor using MRT_irqd_trigger() function.

- MRT_flush_int() runs the handler of the TD-handler, afterwards resets the shower field and the MRT_TDTRIGGER bit in the flag fields of the TDdescriptor.

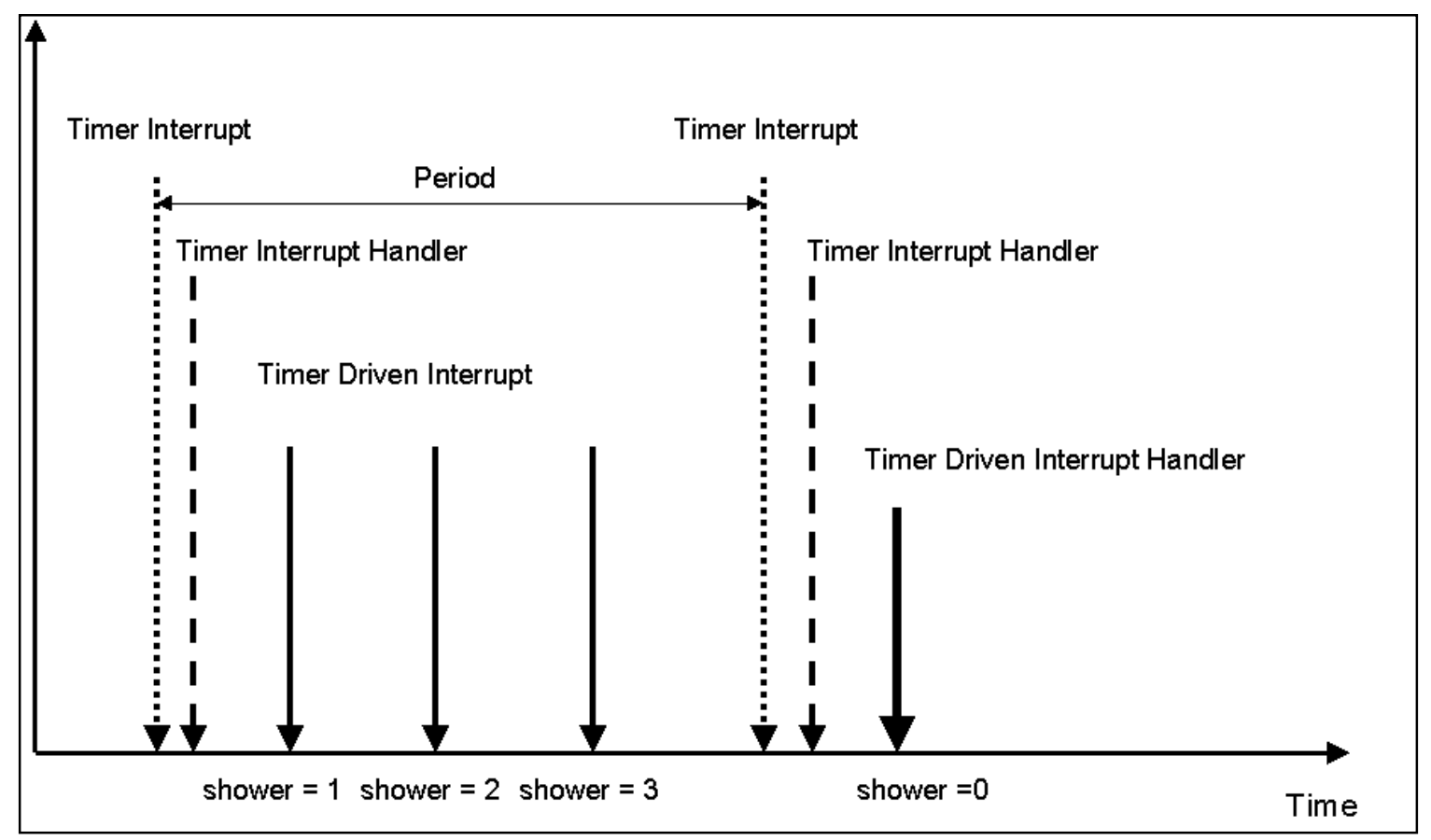

Figure 2.11: Timer Driven Interrupts.

Some devices must be serviced by polling. A software TD-interrupt descriptor can be set associated to a VT with a MRT_ACT_IRQTRIG action type and the period that the device needs. 
The following is a stage of the operations executed:

- On each Timer Interrupt, MRT_IRQ_dispatch() checks for expired VTs. If a VT has expired, the Timer handler is triggered and it will run in the next call to $M R T$ flush_int().

- MRT_flush_int() runs the Timer handler that enqueues expired VTs for later processing of their actions. More details in Chapter 4.

- MRT_flush_int() calls MRT_vtimer_flush() that runs actions of those expired VTs. The action of a VT assigned to a TD-interrupt handler is $M R T \_A C T \_I R Q T R I G$, that triggers the descriptor using MRT_irqd_trigger() function.

- MRT_flush_int() runs the TD-handler that polls the device to check if it needs attention.

\subsubsection{Real-Time Event-Driven Interrupts}

When MRT_IRQ_dispatch() is invoked by a ED-interrupt, it checks if the priority of the interrupted process/handler ( $\left.M R T_{-} s v . p r t y l v l\right)$ is greater than the priority of the interrupt. If it is not, the MRT_IRQ_dispatch() triggers the interrupt descriptor for later processing using the kernel function $M R T$ _irqd_trigger(). Next, when in the next running of $M R T \_f l u s h \_i n t()$, all triggered interrupt descriptors are flushed in priority order.

If the interrupt's priority is greater than $M R T_{-} s v \cdot p r t y l v l$, the interrupt descriptor RThandler is invoked with minimal delay.

\subsection{Preventing Interrupt Priority Inversion}

In RTOS unpredictability is introduced by interrupts from some devices. A type of unbounded priority invertion is produced when a higher priority process is executing and lower priority hardware devices produce an interrupt shower. That showers could only be generated by asynchronous devices like network, USB, parallel or serial port interfaces. Timer interrupts are periodic and the period is set by the kernel. Disks, diskettes and CD-ROMs generate interrupts once they finish with commands commended by the kernel, when the user inserts or removes a removable media, or on error conditions, but never cause a shower. 
MINIX4RT handles interrupts on a preemptive basis; when an interrupt occurs, every execution at lower interrupt levels is suspended and execution begins immediately on the highestlevel request. Processing continues until the highest-level interrupt processing has been completed. This places a responsibility on device drivers writers in that system responsiveness is directly related to how quickly a device driver exits its interrupt routine [22].

Future realeases of MINIX4RT will offer Prioritized Interrupt Disabling. With this feature the kernel changes the PIC mask raising the interrupt level to avoid that equal and lower level interrupts could use system resources delaying higher priority processing [23].

When a device triggers an interrupt of a given priority, the PIC masks from the CPU all interrupts of priority less than or equal to the device interrupt's priority. They become pending. When the interrupt level on the PIC drops below an interrupt's priority, the PIC lets the interrupt proceed to the CPU.

Priorized Interrupt Disable will be implemented using the MRT_sv.mask[] array as was described in Section 2.3.17. Before running interrupt handlers or processes, the RT-kernel checks their priorities and will change the PIC mask to the value stored in MRT_sv.mask[priority]. The $M R T \_s v . m a s k[]$ array is filled by the MRT_irqd_set() kernel function.

\subsection{RT-Process Dispatch Latency}

It is very often that a RT-process is scheduled by an IRQ related with an external event. The RT-Process Dispatch Latency is an important measurament in RTOS and in MINIX4RT is composed by ( see Figure 2.12):

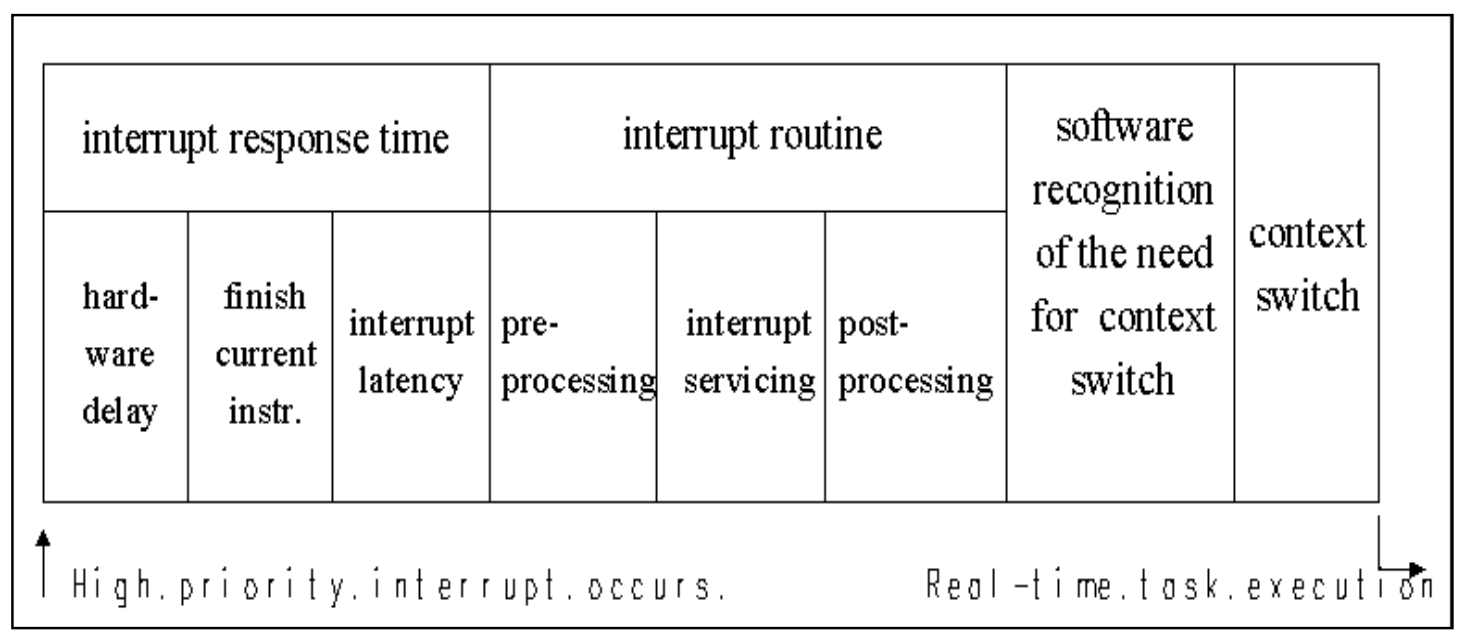

Figure 2.12: RT-Process Dispatch Latency (from [24]). 
- Hardware Delay: Not controlled by the RTOS.

- Finish Current CPU Instruction: Not controlled by the RTOS.

- Interrupt Latency: It is affected by the granularity of the Interrupt Disabling-Interrupt Enabling periods that the RT-kernel use as a mutual exclusion mechanism.

- Preprocessing Time: Includes the processing costs of MRT_IRQ_dispatch() and the top half of HWINTxx functions.

- Interrupt Servicing Time: It is the time consumed by the $M R T$ do_handler() function that includes the handler itself.

- Post Processing Time: It includes the time consumed by the RTscheduler, and the processing costs of the bottom half of HWINTxx function that makes the context switch. 


\section{RT-PROCESS MANAGEMENT AND SCHEDULING}

There are several identifiable approaches to marrying RT and NRT technologies such as the ones listed below [25]:

1. A general purpose OS with added RT-features such as periodic processing and priority-inversion-free IPC mechanisms.

2. A general purpose OS with a dynamically configurable kernel that is capable of accommodating user or application specific process, scheduling, and memory management modules.

3. A subkernel that support coresident OSs partitioning the CPU into two virtual machines running an unmodified general purpose OS and a RTkernel.

Yodaiken [26] proposes a variant of the last approch, where a RT-microkernel threats a timesharing OS as the lower priority task that executes only if there are not any RT-process ready to run. This proposal was adopted for the MINIX4RT kernel and it has the following advantages:

- A clean separation exists between NRT and RT-services.

- The RT-kernel executes in a predictable manner, so it is possible to analyze the conditions under which RT-processes will be guaranteed to be feasible.

- The time sharing OS can function correctly with few modifications.

This chapter describes the composition of RT-processes, their states and transitions, introduces RT-process creation and termination, and gives details about RT-process scheduling. 


\subsection{MINIX4RT Execution Modes}

MINIX4RT starts with the same functionalities as MINIX, therefore it can not run RTprocesses. There must be a NRT-process that invokes a System Call that switches the behavior of the RT-kernel to allow the execution of RT-processes.

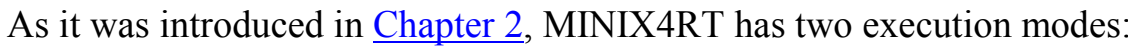

- NRT-mode: Any RT-processes neither RT-interrupt handlers can not run in this mode, therefore the system runs as MINIX does.

- RT-mode: The system runs under the RT-kernel control and RTprocesses and RT-interrupt handlers can be executed sharing the system with NRT-processes and NRT-interrupt handlers.

To switch between those execution modes the mrt_RTstart() and mrt_RTstop() System Calls are provided. They use the services of a new Task named MRTTASK (presented in Chapter 2 and detailled in Chapter 6) that is RT-kernel agent that function as glue among processes and the RTkernel.

\subsection{Real-Time Process Creation}

Only NRT-process can be created and terminated under MINIX4RT. The RT-kernel does not add new System Calls to create RT-processes. On the other hand a NRT-process is converted into a RT-process using the mrt_set2rt() System Call. Before converting a process, several parameters (as priority, period, watchdog, etc.) must be passed to the RT-kernel using the mrt_setproc() System Call.

A RT-process must be converted back into a NRT-process (explained in Section 3.8), using the mrt_set2nrt() System Call, before it can be terminated. When the system runs in NRT-mode, any process that calls $m r t \_s e t 2 r t$ () trying to convert itself into a RT-process, receives an error return code.

As there are two kernels with a shared set of processes with their own process states and transitions each, the bit named MRT_P_REALTIME in the process descriptor status flags (proc [].p_flags) is set for RT-processes.

The MINIX ready) function enqueues a process descriptor into one of the MINIX Ready queues only if all bits of proc[].p_flags are cleared (0x00). With the MRT_P_REALTIME bit set, a RT-process can not be in the READY state, therefore it will be ineligible for the MINIX scheduler. 
As is described by Tanenbaum [2], a MINIX process have 3 basic states (see Figure 3.1):

- READY: The process is ready to run and waiting to be selected by the MINIX process scheduler.

- BLOCKED: The process is blocked because it has done a MINIX System Call using sendrec() kernel function.

- RUNNING: The process is running under the MINIX kernel control.

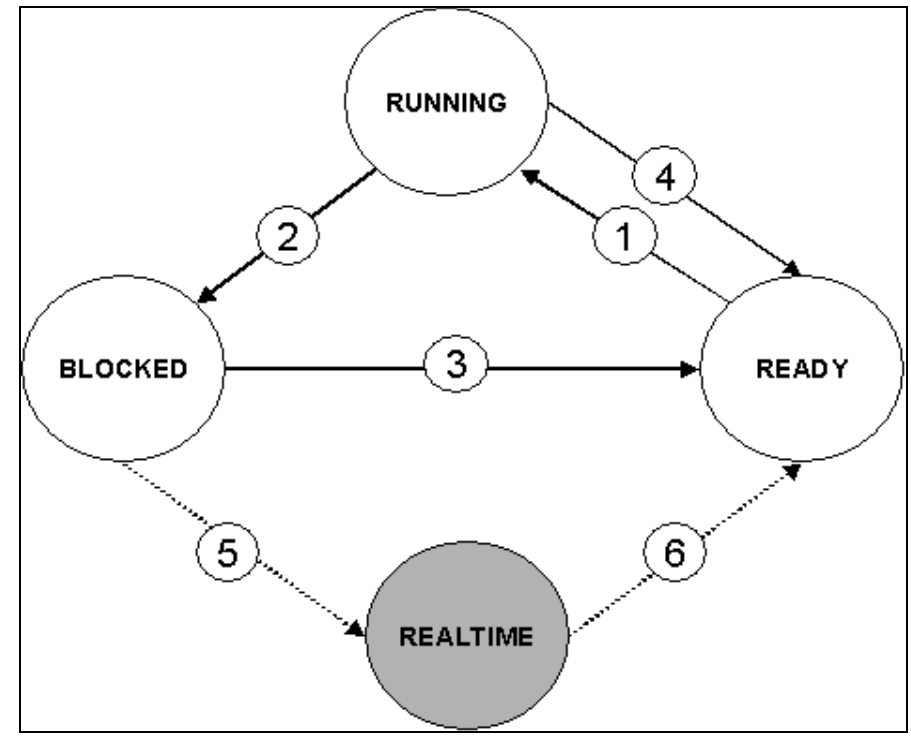

Figure 3.1: NRT-Process States and Transitions.

The following are the process states transitions under MINIX:

1. READY to RUNNING: The process has been selected to run by the scheduler.

2. RUNNING to BLOCKED: The process has done a blocking System Call.

3. BLOCKED to READY: The process has returned from a System Call.

4. RUNNING to READY: The running process has run for its entire alloted timeslice or it has been preempted by other process with higher priority.

When MINIX runs under the RT-kernel control, a fourth process state named REALTIME is added. This state is reached when the NRT-process is converted into a RT-process. The ready() MINIX function is inhibit of inserting the process into the Ready queue, therefore it will be ineligible for the MINIX sheduler. 
Consecuently, the following NRT-process states transitions are the added under MINIX4RT:

5. BLOCKED to REALTIME: The NRT-process has done a mrt_set2rt() System Call converting itself into a RT-process.

6. REALTIME to READY: The RT-process has done a mrt_set2nrt() System Call converting itself into a NRT-process. This transition also occurs when the RT-process calls exit) or when it receives a NRT-signal sent by a NRT-process.

\subsection{RT-Process States and Transitions}

After a NRT-process is converted into a RT-process, it changes from one state to another. The states recognized by the RT-kernel are (Figure 3.2):

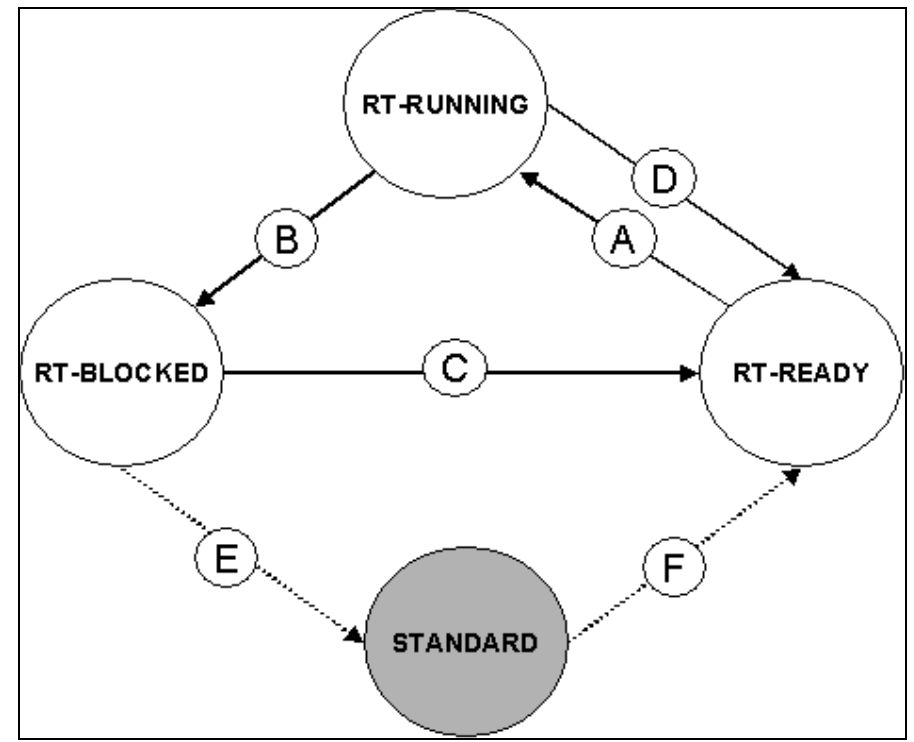

Figure 3.2: RT-Process States and Transitions.

- RT-READY: The RT-process is ready to run and waiting to be selected by the RT-process scheduler.

- RT-BLOCKED: The RT-process is suspended because it has done a blocking RT-System Call to the RT-kernel.

- $\quad$ RT-RUNNING: The RT-process is running under RT-kernel control. 
- STANDARD: The RT-process has been converted into a NRT-process and must be ignored by the RT-kernel.

The RT-process state transitions are:

A. RT-READY to RT-RUNNING: The RT-process has been selected to run by the RT-scheduler.

B. RT-RUNNING to RT-BLOCKED: The RT-process has done a blocking RT-System Call.

C. RT-BLOCKED to RT-READY: The process has returned from a RTSystem Call.

D. RT-RUNNING to RT-READY: The running RT-process has been preempted by other RT-process with higher priority.

E. RT-BLOCKED to STANDARD: The RT-process has done a mrt_set2nrt() System Call to convert itself into a NRT-process. This transition also occurs when the RT-process calls exit() or when it receives a NRT-signal from another NRT-process.

F. STANDARD to RT-READY: The NRT-process has done a mrt_set2rt) System Call to convert it into a RT-process.

Really, STANDARD and REALTIME are compound states. REALTIME is the set of MINIX4RT process states and STANDARD is the set of MINIX process states as it shows in Figure 3.3. 


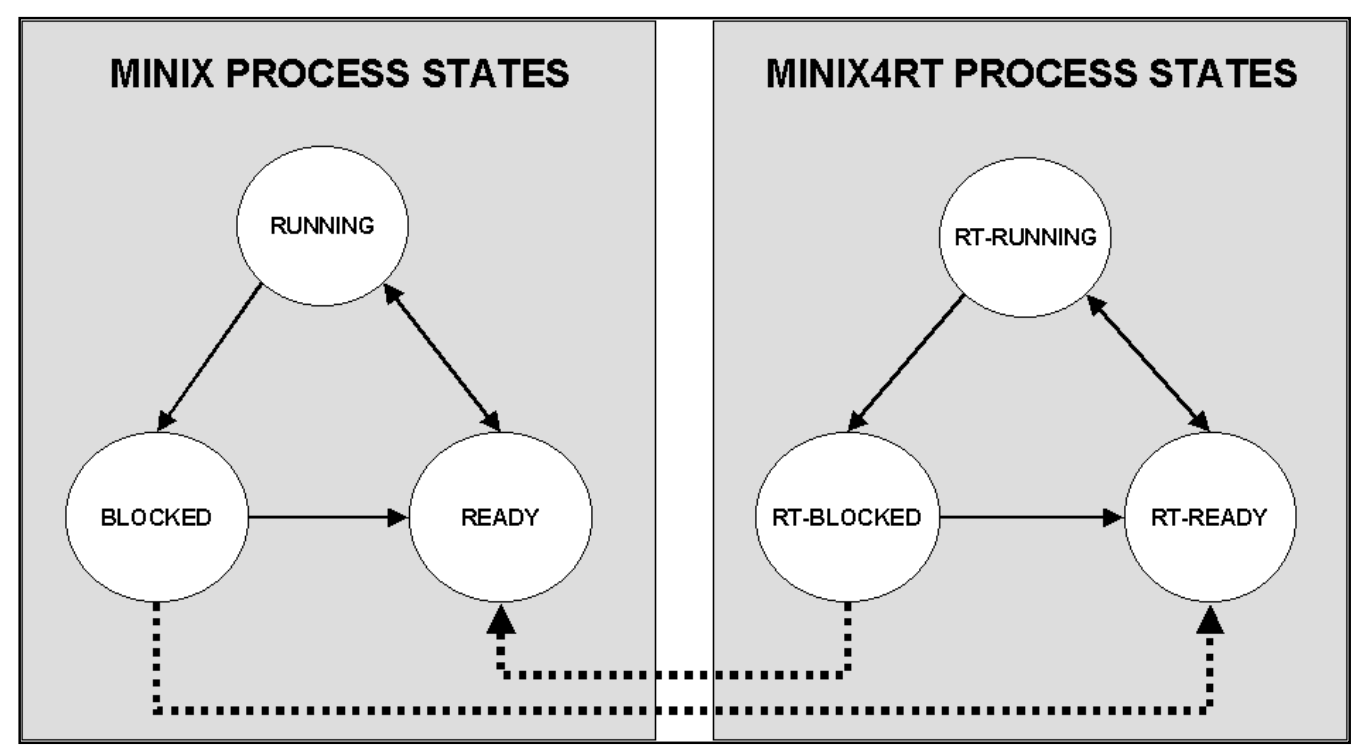

Figure 3.3: RT and NRT Process States and Transitions.

\subsection{Process Descriptor Real-Time fields}

MINIX uses a process descriptor table to keep the description and status information of every process in the system. Each process descriptor has a field named $p_{-}$flags to indicate the reason why a process is blocked. If $p_{f}$ flags $=0$, the process can be scheduled by the MINIX process scheduler.

New fields were added to the process data structure for RT-process management and statistics collection (see Figure 3.4). The data structure of a RT-process is presented in Appendix D.

\begin{tabular}{|c|c|}
\hline \multirow{6}{*}{$\begin{array}{c}\text { Non } \\
\text { Real-Time } \\
\text { Fields }\end{array}$} & flags \\
\hline & priority \\
\hline & baseprty \\
\hline & period \\
\hline & limit \\
\hline & deadline \\
\hline it & watchdog \\
\hline st & \\
\hline *pmq & \\
\hline${ }^{*} \mathrm{pvt}$ & scheds \\
\hline getfrom & mdl \\
\hline sendto & timestamp \\
\hline *pmsg & msgsent \\
\hline${ }^{*} p m h d r$ & msgrcvd \\
\hline *pnextrdy & \\
\hline${ }^{*}$ pprevrdy & \\
\hline
\end{tabular}

Figure 3.4: Process Descriptor Real-Time Fields. 
The RT-process attributes fields are (as part of the $r t$ data structure):

- flags: To keep the RT-process status flags. It has a bit named $M R T P_{-}$REALTIME to distinguish among RT and NRT-processes. The RTkernel accepts RT-System Calls from processes with this bit set (with the exception of mrt_set2rt() System Call). It has another bit named $M R T$ P_PERIODIC to distinguish among periodic and non-periodic RTprocesses. A periodic RT-process performs computation at a regular time interval (period).

- priority: The effective scheduling priority used by the RT-scheduler to select the next RT-process to run. It usage is explained in Section 3.6.

- baseprty: The priority assigned to the process when it is converted to RT. It is used by the Basic Priority Inheritance Protocol (BPIP) to restore the efective priority (explained in Chapter 5).

- period: The scheduling period of a RT-periodic process. It is specified in RT-timer ticks (explained in Chapter 4).

- limit: A limit for the number of RT-schedulings for the process (explained in Section 3.5).

- deadline: The RT-process deadline. It is specified in RT-timer ticks.

- watchdog: The RT-PID (RT process ID defined by mrtpid_t data type) of a RT-process that provides services to protect the RT-process against deadline expiration. The watchdog process can be programmed to perform several actions on the occurrence of a RT-process overrun. When a RTprocess does not complete its work before its deadline expiration, the RTkernel sends a MT_DEADLINE message to the watchdog RT-process specified in the process descriptor.

Other RT-process resource management fields are:

- pmq: A pointer to a message queue assigned to the RT-process (detailled in Chapter 5).

- pvt: A pointer to a VT assigned to the RT-process (only for periodic processes). 
- getfrom: The RT-PID of a RT-process from which the process is waiting to receive a RT-message.

- sendto: The RT-PID of a RT-process to which the process is waiting to send a synchronous message.

- pmsg: A pointer to the message received.

- $\quad$ pmhdr: A pointer to the header of the message received.

- pnextrdy: A pointer to the next ready RT-process descriptor in the RTready queue (explained in Section 3.7).

- pprevrdy: A pointer to the previous ready process descriptor in the RTready queue (explained in Section 3.7).

The RT-process statistical fields (st in Figure 3.4) are explained in Chapter 7.

\subsection{The RT-Process Scheduler}

The process scheduler is the component of the kernel that selects which process to run next. The scheduler can be viewed as the OS component that divides, using a defined policy, the finite resource of CPU time between the runnable processes on a system.

The set of rules used to determine when and how to select which process to run next is called scheduling policy[15]. A scheduler's policy often determines the overall feel of a system and is responsible for optimally utilizing CPU time. The policy behind a RT-scheduler is simple:

"A priority scheduled Real-Time system must ensure that the highest priority runnable process can start to run in a bounded time — and the bound needs to be small." [27].

The first and most common scheduling method in RTOS is preemptive priority-based scheduling, where a lower priority process is preempted by a higher priority process when it becomes ready to run. The RT-scheduler always selects the highest priority runnable RT-process for execution. All unvoluntary context switches are triggered by interrupts. Timer interrupts can cause preemption due to Timer-Driven RT-process activation. If the priority of the activated RT-process is higher than the priority of the currently running process, the execution of current is interrupted and the RTscheduler is invoked to select another RT-process to run. 
The MINIX scheduler is implemented in the pick_proc() kernel function. MINIX4RT modifies its code calling the RT-scheduler (MRT_pick_proc() function) at first. As consecuence, when the MINIX scheduler is invoked, the RT-schedulers runs first. MRT_pick_proc() tries to find the $R T-R E A D Y$ process with the highest priority, returning the pointer to the RT-process descriptor. If there are not such RT-process, the pick_proc() stills running its original code trying to find the highest priority NRT-process.

MRT_pick_proc() also updates system and RT-process scheduling statistics and controls that the number of schedulings of a RT-process does not reach the specified limit. Once the limit is reached, the RT-process is removed from its RT-READY queue. Also, the bit into the flag field named MRT_STOP is set to avoid that the process could run allowing gathering of RT-process statistical information.

The RT-scheduler uses an optimized process-selection algorithm, based on a set of ready queues and a bitmap [28]. Each bit in the bitmap represents a RT-READY queue. If a bit is set, it means that at least one process is RT-READY in that queue. Typically, the bitmap is scanned for the highest priority non-empty queue, and the first process in that queue is selected to run.

The RT-scheduler implements fully $\mathrm{O}(1)$ scheduling. The algorithm completes in constanttime, regardless of the number of RT-READY processes.

In MINIX (and other time-sharing OSs), the timeslice is the numeric value that represents how long a process can run until it is preempted. MINIX4RT does not use a timeslice for preempt a RTprocess. Only a higher priority process can preempt the running process or it must relinquish the CPU by itself.

When a higher priority process enters the RT-READY state, the RT-kernel calls the RTscheduler to find the highest priority RT-READY process to execute (presumably the process that just became runnable).

\subsection{Process Priority}

A common type of scheduling algorithm is priority-based scheduling. The idea is to rank processes based on their worth and need for processor time. Processes with a higher priority will run before those with a lower priority, while processes with the same priority are scheduled round-robin (one after the next, repeating). 


\subsubsection{NRT-Process Priorities}

MINIX uses three Ready queues to schedule processes as it is shown in Figure 3.5:

- TASK_Q: Assigned for I/O Tasks.

- SERVER_Q: Assigned for Servers like Memory Manager $(M M)$ and File System Manager $(F S)$.

- USER_Q: Assigned for User-level processes.

The MINIX scheduling algorithm is simple. It looks up for a process into the READY queues starting with the $T A S K \_Q$, next continues with the $S E R V E R \_Q$, and finally with the USER_Q. The scheduler selects the first process it finds in the non-empty highest priority queue. If all queues are empty, the IDLE process (detailled in Chapter 7) is scheduled.

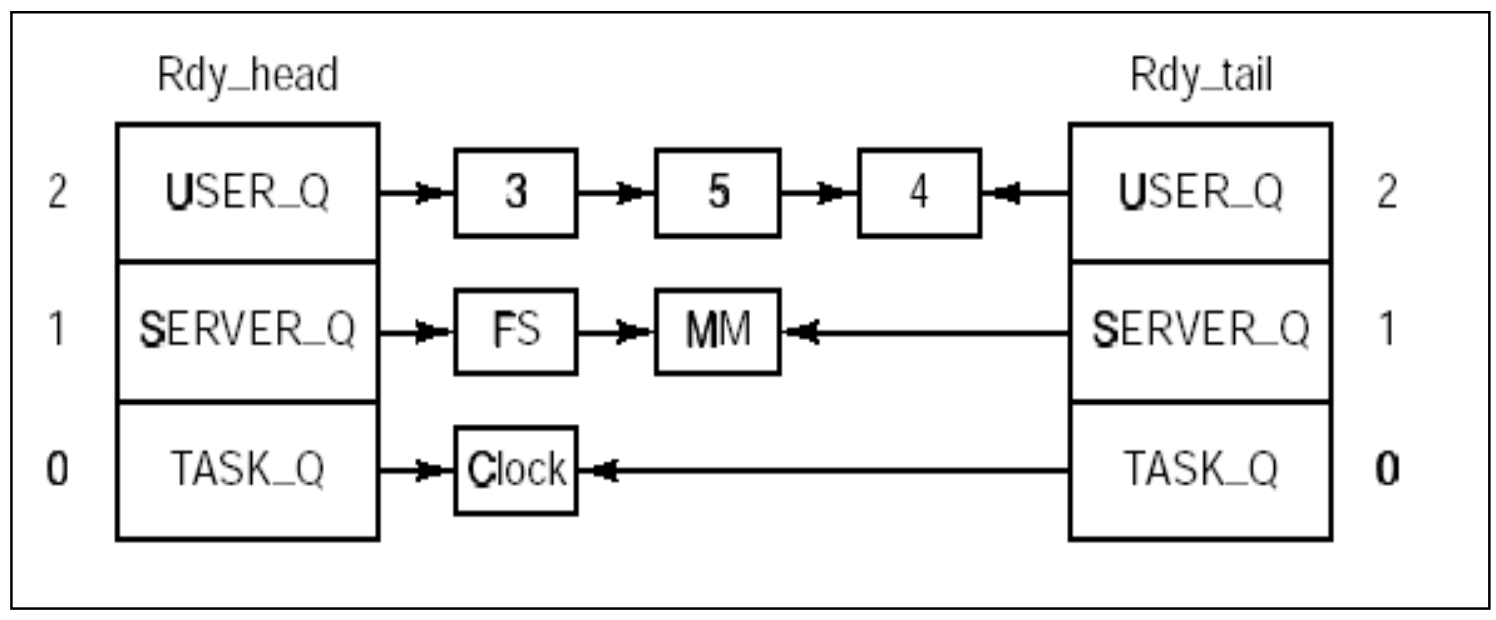

Figure 3.5: MINIX READY Queues (from [2]).

\subsubsection{RT-Process Priorities}

Each RT-process has a base priority (the baseprty field) and an effective priority (the priority field), and is scheduled in accordance with the latter. The base priority of a RTprocess is stablished using the mrt_setproc() System Call before calling mrt_set2rt) System Call that convert the NRT-process into a RT-process. Both, the baseprty and the priority field can have a value ranging from MRT_PRIHIGHEST (0x00) to MRT_PRILOWEST (0x0F).

Normally, the effective priority of a RT-process equals its base priority but, it may be changed by the BPIP (more details in $\underline{\text { Chapter 5) }}$ ) or the scheduling policy. 
The set of RT-ready queues is actually implemented as 16 (MRT_NR_PRTY) separate queues, assigning one queue for each priority. A ready RT-process could be inserted into the RT-queue corresponding to its effective priority field in FIFO or LIFO order. The first process descriptor in the highest RT-ready queue will be selected to run by the RT-scheduler.

The priority field is also used by the RT-kernel to minimize the Interrupt Blocking time. Only those RT-interrupt handlers with higher priorities are executed while the current RT-process is running.

\subsection{RT-Ready Queues Management}

To manage RT-ready queues the RT-kernel have the following data structures:

A set of RT-ready queues, one queue for each priority level (RTM_sp.rdyQ.procL[]).

A bitmap that have one bit assigned for each priority. Initially, all the bits are cleared indicating that all queues are empty (RTM_sp.rdyQ.bitmap).

When a RT-process becomes runnable (that is, its state becomes RT-READY), the corresponding bit to the process priority is set in RTM_sp.rdyQ.bitmap, and the process descriptor is appended to the RT-ready queue in accordance with its priority field.

Finding the highest priority RT-process on the system is therefore only a matter of finding the first bit set in RTM_sp.rdyQ.bitmap. Because the number of priorities is fixed, the time to complete a search is constant and unaffected by the number of running processes on the system.

Each ready queue descriptor $\left(M R T \_\right.$procL_t $)$have one pointer to the first process descriptor and other pointer to the last process descriptor in the queue (see Figure 3.6).

A process descriptor can be inserted into a queue in FIFO or LIFO order. Processes of the same priority will be managed under a FIFO policy, but sometimes a process that inherits its priority by the BPIP must be inserted into a queue in LIFO order (explained in $\underline{\text { Chapter 5). }}$ ).

Each queue descriptor also contains a field named in $Q$ that counts the current number of runnable RT-processes in the queue and a field named $\operatorname{maxin} Q$ that keeps the highest value of the in $Q$ field for statistics.

The following kernel functions help to manage RT-ready queues: 
- MRT_rdyQ_app(): Appends a process descriptor at the tail of a RTready queue.

- MRT_rdyQ_ins(): Inserts a process descriptor at the head of a RT-ready queue.

- MRT_rdyQ_rmv(): Removes a process descriptor from a RT-ready queue.

Several kernel functions operate on RT-ready queues:

- MRT_pick_proc(): The RT-scheduler searchs for the highest priority ready-to-run RT-process.

- MRT_inherit(): RT-IPC could change the priority of the destination process of a message. This function removes a process descriptor from its current RT-ready queue, inserting it into its inherited RT-ready queue using $M R T \_r d y Q_{-} r m v()$ and $M R T \_r d y Q \_i n s()$ RT-kernel functions.

- MRT_disinherit(): Used to change the priority of a RT-process on mrt_reply() System Call. This function removes the processs descriptor from the its current RT-ready queue ( $\left.M R T_{-} r d y Q_{-} r m v()\right)$, and inserts the descriptor into its inherited or base priority RT-ready queue in accordance to the BPIP (MRT_rdyQ_ins()). 


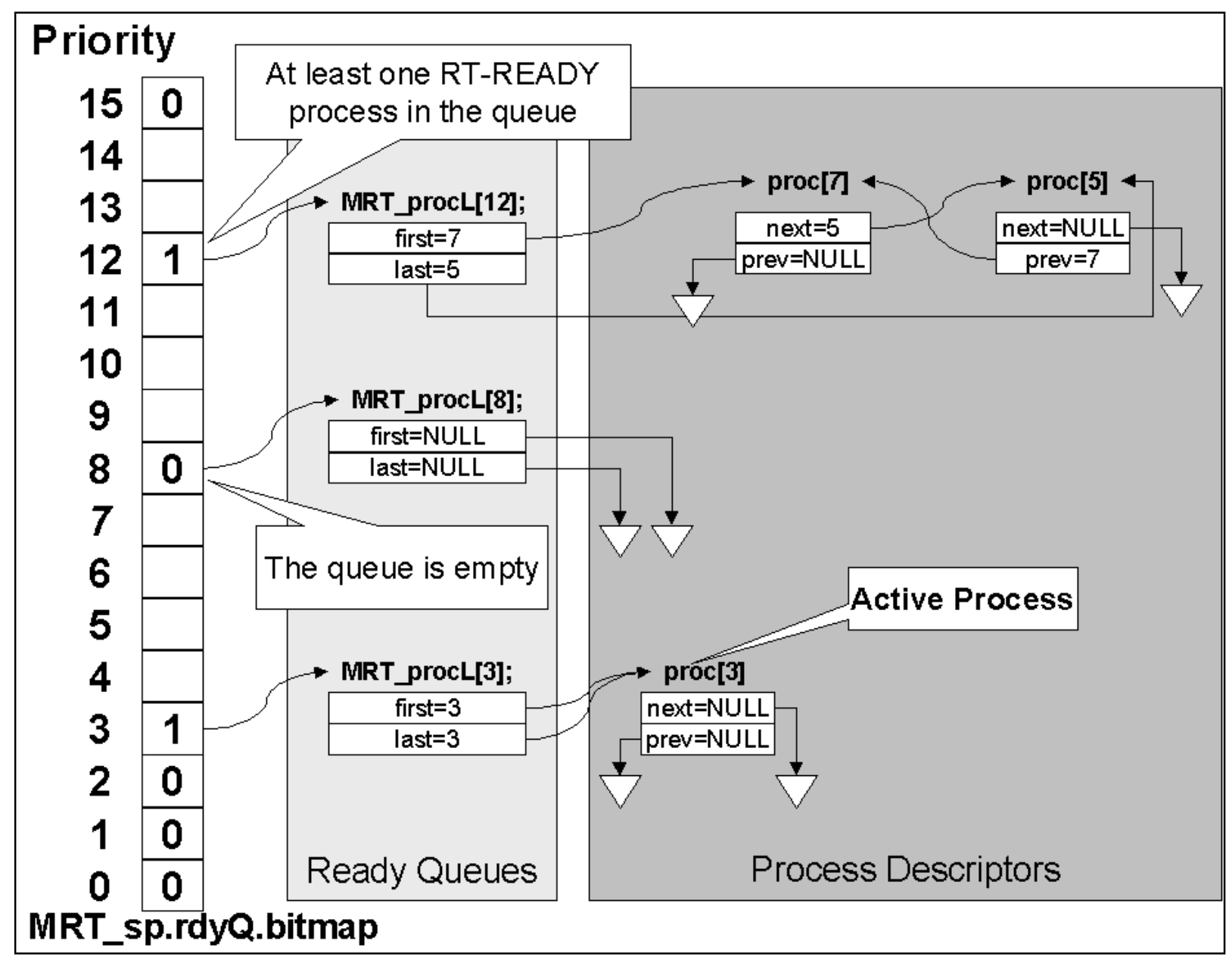

Figure 3.6: RT-kernel Priority Queues.

As it can be seen, there are similarities between interrupt descriptors queues and process descriptors RT-ready queues, but there are considerable differences in the queue usage. As it was described in Chapter 2, once an interrupt descriptor is set, it is inserted into an interrupt descriptor queue. The queue keeps the descriptor even though it has been triggered and its has been serviced. The interrupt descriptor can only be removed by the programmer using the MRT_free_irqd() RTkernel function. RT-ready queues keep only process descriptors in RT-READY and RT-RUNNING states. Process descriptors in other states are removed. As the BPIP could change the process priority, a descriptor could be removed from one RT-ready queue and inserted into other.

MINIX4RT does not have a policy to assign process priorities, but it is easy to create a System Call that set a Rate Monotonic[29] priority schema using the period field of process descriptors or a Deadline Monotonic [30] using the deadline field of process descriptors. Dynamic priority algorithms as Earliest Deadline First (EDF) [31] or Least Laxity First (LLF) [32] requires more complex changes. 


\subsection{RT-process Termination}

MINIX has four ways to terminate a running process:

- Normal Exit: Invoking the exit() System Call by the process itself (voluntary).

- Error Exit: The process discovers a fatal error and invokes the exit) System Call (voluntary).

- Fatal Error: The OS discovers a process fatal error (often a program bug) and terminates the process using the do_kill() kernel function (unvoluntary).

- Killed: Other process sends an uncached signal to the process (unvoluntary).

When MINIX4RT runs in RT-mode, a RT-process must be converted into a NRT-process before it can be terminated.

\subsubsection{RT-process Termination Using the exit() System Call}

As it will be explained in Chapter 5, RT-processes can not send/receive messages using standard send()/receive() MINIX primitives. If a RT-process calls send() or receive() funcions, it receives an error code on function return.

The exit() System Call uses sendrec(), a single function for doing a send() followed by a receive(). If a RT-process invokes exit(), it should return with an error, but the exit() System Call must not return to the calling process. To overcome this issue, two functions were modified in MINIX4RT as it described below:

- The _exit() Library function invokes mrt_set2nrt() before doing its standard work. The RT-kernel converts the RT-process into a NRT-process, and releases the resources owned by the terminanting process (VTs and a message queue (MQ) (explained in Chapter 5)).

- The exit() function of the MM Server that requests the MRTTASK to release the VTs and the MQ owned by the RT-process. 


\subsubsection{RT-process Termination Using the signal() System Call}

If a NRT-process sends a signal to a RT-process, the target is converted into a NRTprocess before it can receive the signal. That conversion is accomplished by the modified sig_proc() function of the MM. The MM sends a MRT_STDSIGNAL message to MRTTASK returning without any action if the target is a NRT-process or converting the target into a NRT-process before return.

If the signal sent is uncached by the process, the process will exit releasing RTresources before.

\subsubsection{Releasing RT-process Resourses and Housecleaning}

To keep the system concistency, the RT-kernel (MRT_proc_stop() function) carry out some housecleaning tasks during the the conversion of a RT-process into a NRT one. It releases RT-resources owned by the converted process as is described below:

- Any RT-process with pending requests to the converted process will return with $E \_M R T \_E X I T I N G$ error code.

- A MT_SIGNAL message is sent to the watchdog RT-process of the converted process.

- A MT_SIGNAL message is sent to all RT-process with their watchdog field equals to the converted process. The watchdog fields of that RTprocesses are reset.

- All watchdog fields of RT-interrupt descriptors that equals to the converted process are reset.

- All pending asynchronous messages sent by the converted process to other RT-processes are removed from their MQs.

- The VT owned by the process related to a converted periodic process is stopped (but not released).

- The VT owned by the process related to IPC timeout management is stopped (but not released). 


\section{TIME MANAGEMENT}

\subsection{Timing Mechanisms}

Timers are mechanisms that are able to notify the kernel or user programs that a certain interval of time has elapsed [15], they play an integral role in RT-systems [ $\underline{33}, \underline{34}]$.

RT-applications must be able to operate on data within strict timing constraints in order to schedule application or system events. Timing requirements can be in response to the need for either high system throughput or fast response time. Applications requiring high throughput may process large amounts of data and use a continuous stream of data points equally spaced in time.

The following types of timing mechanisms are often used:

- A pause() function: A function pause() is used to suspend the active process for a specified time. As is explained in [33], an inaccuracy could occur because the pause function use the Timer interrupt as its time base, and it depends on the Timer resolution.

- Recovering from Message Loss: Usually a timer is kept while awaiting for a message. If the message is received, timer is stopped. If the timer expires, message loss is registered. In such a case, a retry logic is implemented by restarting the timer and awaiting for the message again. If the number of retries reaches a threshold, the activity is aborted and appropriate recovery action is initiated.

- Recovering from Software Faults: Whenever a feature is initiated, a feature wide timer is kept to ensure feature success. If some software or hardware module involved in the feature hits recovery, the feature will fail and the timer expiry will be the only method to detect the feature failure. On 
expiry of the timer, the feature may be reinitiated or recovery action might be taken.

- Sequencing Operations: Timers are used for sequencing time based state transitions.

- Polling: A timer is kept and the system polls for a condition on every timeout.

- Periodic Operations: For implementing audits, periodic timers are kept. On each timer expiry, software audit is initiated.

- Failure Detection: For monitoring the health of other modules, a module runs a timer. It expects a sanity message periodically from all the other modules before the expire of the timer. If certain number of sanity messages are missed in succession from a module, module failure is declared as failed.

- Inactivity Detection: Timers are also used for detecting the inactivity in a session.

\subsection{MINIX4RT Timer Interrupts}

MINIX use three software components to handle time related tasks.

- The Clock ISR: increments de real time counter, decrements the quantum of the running process and checks it for zero, makes CPU accounting, and decrements an alarm counter.

- The Clock Task: It is a Task that is scheduled when a Timer interrupt occurs and there is work to do, such as when an alarm must be sent or a process has run too long.

- The Synchronous Alarm Task: It is a Task to send messages (synchronous events) directly to the server that requested the synchronous alarm, which must be waiting for the message. Synchronous alarms can only be requested by servers, for example, the network server wanting to time out if an acknowledgement packet does not arrive in a certain amount of time. 
MINIX4RT need to enhance Timer operations accuracy, resolution and predictability needed by a hard Real-Time OS, therefore it does not use any of the described components because they implies several context switches. This approach also facilitates software updates when new versions of MINIX will be realeased because the added source code is less intrusive.

Several time-keeping activities are triggered by interrupts raised by the Programmable Interval Timer (PIT) on IRQ line 0. Some of these activities need to be executed as soon as possible after the interrupt is raised, while the other are delayed out of interrupt time [15].

The RT-kernel carry on the following actions related to time:

- Updates the tick count since the RT-mode startup.

- Checks whether the interval of time associated with each timer has elapsed.

- Executes actions related to expired timers.

- Emulates a Timer interrupt for the MINIX kernel.

- Keeps updated an ordered timer list.

- Inserts and removes timers from free and expired lists.

The following RT-kernel functions deal with important Timer interrupts related activities:

- MRT_irq_dispatch(): As was explained in Chapter 2, this function is executed on each hardware interrupt. On each Timer interrupt it takes the following actions:

- It updates the counter MRT_sv.counter.ticks (explained in Section 4.4).

- It copies the MRT_sv.counter.ticks in the Timer interrupt descriptor timestamp field.

- It checks for VT expirations.

- If the Active VT queue is empty or any VT has expired, it reenables the Timer IRQ line before returns. 
- If at least one VT has expired, it triggers the Timer interrupt descriptor (its handler is $M R T \_$clock_BH()) for delayed processing, and reenables the Timer IRQ before returns.

- MRT_clock_BH(): It is the Timer interrupt (Clock in MINIX terminology) Botton Half. It is executed only if a VT has expired outside interrupt time called by MRT_flush_int() and it has the PRI_HIGHEST priority. Its purpose is to change expired VTs from the Active Queue to the Expired Queue (explained in Section 4.8.2).

- MRT_vtimer_flush(): This function, also called by MRT_flush_int(), executes Virtual Timer asociated actions (explained in Section 4.8.3).

It is important to note that the Timer ISR merely indicates that at least a VT has expired. Before the RT-kernel returns from interrupt, the Active VT queue will be checked for expired VTs and the their actions will be executed.

\subsection{MINIX Virtual Timer Interrupts}

One important use of VTs is the emulation of Timer interrupts for the MINIX kernel. After executing mrt_RTstart() System Call, a VT and a software interrupt are assigned to emulate Timer interrupts for MINIX. The RT-handler field of the software interrupt descriptor points to the original clock_handler() function of MINIX kernel.

IBM-compatible PCs include a device called 8253/4 Programmable Interval Timer (PIT). The PIT is programmed by the kernel so that they issue interrupts at a fixed, predefined frequency. These periodic interrupts are colled Timer ticks and are crucial for implementing the timers used by the kernel and user's programs. This device issues a special interrupt on IRQ0 called Timer interrupt, which notifies the kernel that a time interval has elapsed. Modern CPUs have timers in the local APICs.

As the frequency of Timer interrupts of MINIX4RT could be greater than in MINIX, the RTkernel emulates MINIX Timer interrupts invoking the standard timer handler (the clock_handler() function) at lower or equal frequency than the PIT.

The Timer interrupt rate of standard MINIX is defined as a constant in $H Z$ as follow:

$$
\text { \#define HZ } 60 \quad / * \text { clock freq (software settable on IBM-PC) */ }
$$


MINIX4RT redefines $H Z$ as follow to make more easy the use of integer decimal periods:

$$
\text { \#define HZ } \quad 50 \quad / * \text { clock freq (software settable on IBM-PC) */ }
$$

The Timer interrupt rate of MINIX4RT is established by the MRT_sv.tickrate system global variable. $H Z$ and $M R T \_s v$.tickrate must be harmonic frequencies to virtualize Timer interrupts for the MINIX kernel only when Timer interrupts occur, therefore they are related by an integer value:

$$
\text { MRT_sv.tickrate }=\text { MRT_sv.harmonic * HZ; / } * \text { MRT_sv.harmonic }=1,2,3, \ldots . . \mathrm{N} * /
$$

To preserve the illusion of the standard MINIX tick rate $H Z$, the MINIX interrupt handler clock_handler() is called after MRT_sv.harmonic timer interrupts. This interrupt rate is emulated using a VT with period $=M R T \_s v$.harmonic and MRT_ACT_IRQTRIG action type (See Figure 4.1).

A software interrupt related to the VT is used to defer the processing of the MINIX Timer interrupt handler with a MRT_PRILOWEST priority. On each VT expiration, the software interrupt is triggered and its handler is executed by $R T M \_f l u s h \_i n t()$ function.

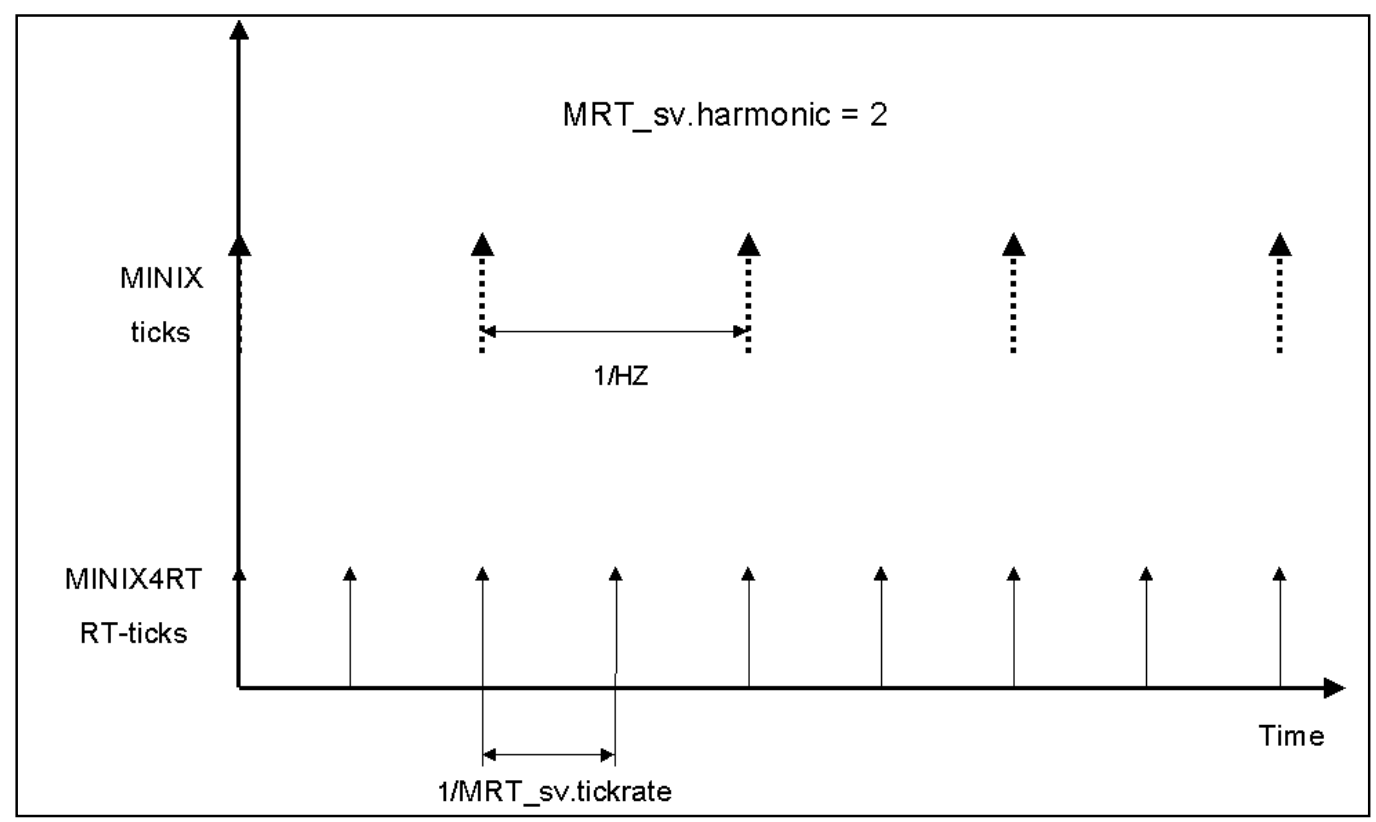

Figure 4.1: MINIX Virtual Timer Interrupt.

\subsection{Timer Resolution}

The 8253/4 PIT uses an internal oscillator frequency (TIMER_FREQ) at 1,193,180 Hz. It has a 16 bits $L A T C H$ register to set the ratio between the oscillator frequency and the number of interrupts per second $($ tick_rate $=1193180 / \mathrm{LATCH})$. 
Only some values of $L A T C H$ issues integer values of tick_rate as it is show in Table 4.1. Therefore, other values of $L A T C H$ result in accuracy error.

Table 4.1: Integer Values of tick_rate.

\begin{tabular}{|c|c|}
\hline LATCH & tick_rate \\
\hline 1 & 1193180 \\
\hline 2 & 596590 \\
\hline 4 & 298295 \\
\hline 5 & 238636 \\
\hline 10 & 119318 \\
\hline 20 & 59659 \\
\hline 59659 & 20 \\
\hline
\end{tabular}

The value of the PIT LATCH is stored in a system global variable named MRT_sv.PIT_latch and it is initilized as:

$$
M R T \_s v . P I T \_l a t c h=T I M E R \_F R E Q / M R T \_s v . t i c k r a t e .
$$

An example illustrates the accuracy error. For a tick rate of 100 [interrupts/s]:

$$
\text { MRT_sv.PIT_latch }=1193180 / 100=11931 \text { and a a reminder of } 80 \mathrm{~Hz}
$$

The reminder represents an additional Timer interrupt every $(11931 * 100 / 80)=15000$ Timer ticks or $150[\mathrm{~s}]$. The RT-Timer interrupt frequency is $100.0067052217[\mathrm{~Hz}]$ and the RT-Timer period is 0.009999329522788 [s] resulting in an error in time accuracy of 0.0000670478 . Table 4.2 shows some RT-interrupt frequencies, RT-Timer periods and resulting errors.

As $M R T$ sv.tickrate is a multiple of $H Z$, it could be changed setting $M R T$ sv.harmonic using mrt_restart(). This System Call can only be used before running any RT-process or when there are not any RT-process running, otherwise all system time reference (in Timer ticks units) would be erroneous (i.e. periods, timestamps, tick counters, etc.).

MINIX kernel keeps tracks of the number of elapsed (virtual) Timer ticks since the system was started in the global system variable named realtime. It is set to 0 during kernel initialization.

MINIX4RT kernel keeps tracks of elapsed (real) Timer ticks since the last mrt_RTstart) or mrt_restart() System Call invocation in the global system variable named MRT_sv.counter.ticks. It is set to 0 during RT-system initialization and incremented by one unit when a Timer interrupt occurs, that is on every Timer tick. Since MRT_sv.counter.ticks is an unsigned 32 bits integer, the time that makes it overflow depends on the tick rate. However, the RT-kernel handles the overflow using another kernel variable named $M R T \_s v$.counter.highticks. 
Note that since the system updates $M R T_{-}$sv.counter.ticks and MRT_sv.counter.highticks, only the former is loaded in the timestamp field of descriptors (interrupt descriptors, process descriptors, message descriptors, etc). The next versions of MINIX4RT will include the extension of the timestamp field.

Table 4.2: Period Errors.

\begin{tabular}{|c|c|c|c|c|c|c|}
\hline $\begin{array}{c}\text { Configured } \\
\text { TickRate } \\
{[\text { int/s }]}\end{array}$ & $\begin{array}{c}\text { Configured } \\
\text { Specified } \\
\text { Period } \\
{[\mathrm{s}]}\end{array}$ & Latch & $\begin{array}{c}\text { Reminder } \\
{[\mathrm{Hz}]}\end{array}$ & $\begin{array}{c}\text { Real Tick } \\
\text { Rate } \\
{[\mathrm{int} / \mathrm{s}]}\end{array}$ & $\begin{array}{c}\text { Real Period } \\
{[\mathrm{s}]}\end{array}$ & $\begin{array}{c}\text { Period } \\
\text { Error }\end{array}$ \\
\hline 100 & 0.010000000 & 11931 & 80 & 100.006705 & 0.009999330 & 0.000067048 \\
\hline 200 & 0.005000000 & 5965 & 180 & 200.030176 & 0.004999246 & 0.000150857 \\
\hline 500 & 0.002000000 & 2386 & 180 & 500.075440 & 0.001999698 & 0.000150857 \\
\hline 1000 & 0.001000000 & 1193 & 180 & 1000.15088 & 0.000999849 & 0.000150857 \\
\hline 1500 & 0.000666667 & 795 & 680 & 1500.85535 & 0.000666287 & 0.000569906 \\
\hline 2000 & 0.000500000 & 596 & 1180 & 2001.97987 & 0.000499506 & 0.000988954 \\
\hline 3000 & 0.000333333 & 397 & 2180 & 3005.49118 & 0.000332724 & 0.001827050 \\
\hline 4000 & 0.000250000 & 298 & 1180 & 4003.95973 & 0.000249753 & 0.000988954 \\
\hline 5000 & 0.000200000 & 238 & 3180 & 5013.36134 & 0.000199467 & 0.002665147 \\
\hline 7500 & 0.000133333 & 159 & 680 & 7504.27673 & 0.000133257 & 0.000569906 \\
\hline 10000 & 0.000100000 & 119 & 3180 & 10026.7227 & 0.000099733 & 0.002665147 \\
\hline
\end{tabular}

Table 4.3 shows some wrap around time (in days) of MRT_sv.counter.ticks for some Timer interrupt frequencies.

Table 4.3: MRT_sv.counter.ticks Overflow Time.

\begin{tabular}{|c|c|}
\hline $\begin{array}{c}\text { MRT_sv.tickrate } \\
\text { [interrupt/s] }\end{array}$ & $\begin{array}{c}\text { MRT_sv.counter.ticks } \\
\text { Days to Overflow }\end{array}$ \\
\hline 50 & 994 \\
\hline 100 & 497 \\
\hline 200 & 249 \\
\hline 500 & 99 \\
\hline 1000 & 50 \\
\hline 1500 & 33 \\
\hline 2000 & 25 \\
\hline 3000 & 17 \\
\hline 4000 & 12 \\
\hline 5000 & 10 \\
\hline 7500 & 7 \\
\hline 10000 & 5 \\
\hline
\end{tabular}

\subsection{3/4 Programmable Interval Timer Programming}

The 8253/4 Programmable Timer provides three independent 16-bit counters called Timer channels that can count in binary or BCD. It can run in one of the six programmable modes: 
- Mode 0: Interrupt on Terminal Count.

- Mode 1: Programmable One-shot.

- Mode 2: Rate Generator.

- Mode 3: Square Wave Rate Generator.

- Mode 4: Software Triggered Strobe.

- Mode 5: Hardware Trigger Strobe.

The programming of a Timer channel is initiated by writing a control word into the control register port at $43 \mathrm{H}$. The control word has the format shown in Figure 4.2:

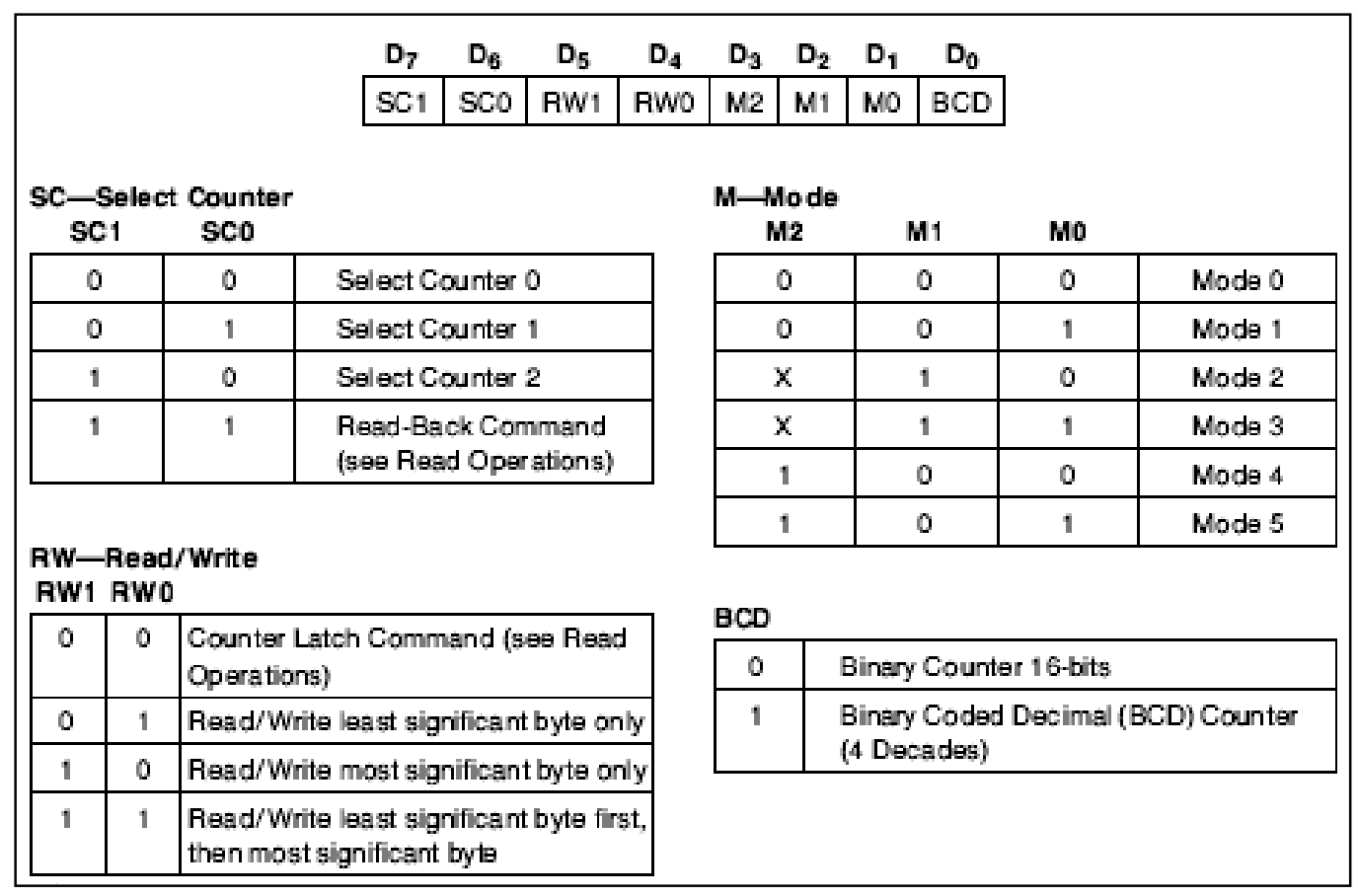

Figure 4.2: PIT Control Word Format.

Since the counters are 16 bits long but the I/O port through which data is transferred is only 8 bits long, two data transfer operations to fill the whole counter.

Setting the bits D5 and D4 to 11 in order to load first the LSB then the MSB as the preset 16bit word count for the corresponding counter. Here are some relevant I/O port addresses:

- $\quad 40 \mathrm{H}$ Timer Channel 0 Counter

- $\quad 41 \mathrm{H}$ Timer Channel 1 Counter 
- $\quad$ 42H Timer Channel 2 Counter

- $43 \mathrm{H}$ Timer Control Register

Two RT-kernel functions operates on the PIT Channel 0:

- MRT_set_timer(): Sets the Timer interrupt rate with a frequency of ( $H Z$ *harmonic). The function argument is the harmonic frequency of the MINIX Timer frequency.

- MRT_read_timer(): Reads the current value of the PIT counter.

\subsection{Estimating the Timer Interrupt Latency}

When the PIT counter reachs zero, the PIT raise IRQ 0 and resets the counter to the value in the LATCH. As the PIT remains decrementing the counter, during the execution of the Timer interrupt handler, the value of the counter lets compute the latency of the handler (See Figure 4.3).

Timer_Handler_Latency $=$ MRT_sv.PIT_latch - MRT_read_timer()

To consider the overhead of executing MRT_read_timer(), the kernel estimates its value at initialization time and stores the result (in PIT $\mathrm{Hz}$ units) into the system variable MRT_sv.PIT_latency.

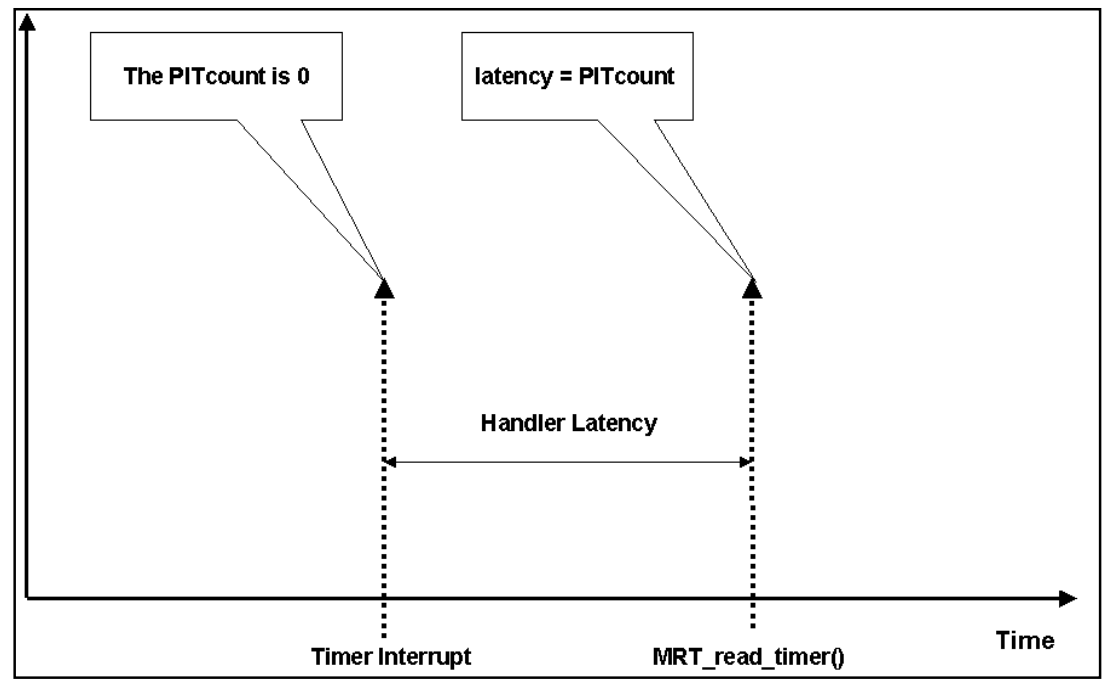

Figure 4.3: Estimating Timer Interrupt Latency. 
Applications can get the estimated Timer Interrupt Latency using the mrt_getistat() System Call for IRQ 0, and the value of MRT_sv.PIT_latency using mrt_getsval() System Call (more details in Appendix A).

\subsection{Real-Time and Non Real-Time Timer Handlers}

At startup, the system is not ready for RT-processing (described in Chapter 3). All interrupt handlers used by the kernel are MINIX NRT-handlers, including the Timer handler that is the function clock_handler(). When the RT-mode starts, the system enables the use of RT-handlers for those interrupt descriptors defined as Real-Time (the bit $M R T \_R T I R Q$ set in the flag field), including the RT-Timer handler named MRT_clock_BH().

Sometimes, it is necesary to reconfigure the system to change some parameters without recompilation and restarting it. The mrt_restart() System Call can be used to change RT-processing parameters and reset system statistics. One of this parameters is the Timer interrupt frequency, and it could be changed using the harmonic argument of mrt_restart(). All statistical fields of data structures are reset because changing the Timer period causes that they will be erroneous as it was explained in Section 4.4.

\subsection{Virtual Timers}

A Virtual Timer (VT) is a software facility that allows to take an action at some future moment, after a given time interval has elapsed; a time-out denotes a moment at which the time interval associated with a timer has elapsed [15].

Some systems use VTs to handle periodic processing. Once the period of the VT has elapsed, the periodic process is scheduled, the VT is removed from a queue and inserted in other position of the queue depending on the period. This approach presents significant overhead to the periodic process and particulary in those that have small periods.

MINIX4RT uses VTs to manage periodic processing and other related activities. It has NR_VTIMERS Virtual Timers descriptors data structures defined in kernel space.

A VT descriptor has the MRT_vtimer_t data structure (see Appendix D) with the following fields:

- period: The period of the VT in RT-ticks. 
- nextexp: The number of RT-ticks for the next VT expiration in the queue (explained in Section 4.8.2).

- limit: The number of expirations until free the VT. A limit of 0 means that the VT has no expiration limit.

- action: The code of the action to execute on expiration (explained in Section 4.8.1).

- param: A generic integer used as a parameter for the action on VT expirations. The param field enables to define a single general-purpose function that handles the time-outs of several device drivers; the param field could store the device ID or other meaningful data that could be used by the function to differentiate the device.

- index: The VT identification number.

- owner: The VT RT-process owner.

- priority: The VT owner's priority. This field is used on VT expirations to run actions in priority order.

- timestamp: The last expiration timestamp.

- expired: Counts the number of VT expirations.

- next: A pointer to the next VT in the queue.

- prev: A pointer to the previous VT in the queue.

\subsubsection{Virtual Timers Handling Functions}

The RT-kernel functions that handle VTs are:

- MRT_vtimer_flush(): Searches for expired VTs with equal or greater priorities than the MRT_sv.prtylvl and runs their actions as was explained in Chapter 2.

- MRT_vtimer_alloc(): Allocates a VT with the parameters passed as arguments. 
- MRT_vtimer_free(): Reset all parameters of a VT and inserts it into the VT Free queue.

- MRT_vtimer_ins(): Inserts an VT into the Active/Expired/Free queue.

- MRT_vtimer_rmv(): Removes a VT from the Active/Expired/Free queue.

- MRT_vtimer_search(): Searches for a VT in a queue.

- MRT_vtimer_run(): Executes a VT action. Once the action has been executed, the VT is reescheduled for the next period (if expired $>=$ limit) or it is released to the Free VT queue. The VT actions could be:

- MRT_ACT_NONE: No action is executed.

- MRT_ACT_PERIODIC: Used for periodic processes. This action wakes up the VT owner process.

- MRT_ACT_MSGOWN: Sends a MT_TIMEOUT message to the VT owner process.

- MRT_ACT_MSGWDOG: Sends a MT_TIMEOUT message to the VT owner's watchdog process.

- MRT_ACT_IRQTRIG: Trigger an IRQ descriptor specified in the param field.

- MRT_ACT_SNDTO: A send type timeout has expired (see Chapter 5).

- MRT_ACT_RCVTO: A receive timeout has expired (see Chapter 5).

- MRT_ACT_WAKEUP: This action wakes up the VT owner process if it has called the mrt_sleep() System Call that put the process into the $R T$ $B L O C K E D$ state.

- MRT_ACT_SCHED: This action wakes up the process specified in the param field.

- MRT_ACT_DEBUG: Used for debugging purposes. Its prints the param field in console. 


\subsubsection{Virtual Timers Queues}

The RT-kernel uses several queues for handling VTs. The queues are:

- MRT_st.timerQ: It is the queue for Active VTs ordered by expiration time (see Figure 4.4). The firstexp field of the queue counts the number of RT-ticks for the expiration of the first VT. Each VT has a field named nextexp that counts the number of RT-timer ticks for the expiration of the next VT in the queue. Once a VT has expired, it is removed from this queue.

- MRT_st.freeQ: It is the queue for Free VTs.

- MRT_st.exp.expiredQ[]: It is an array of queues of expired VTs with pending actions. There is one queue for each system priority. Once the action of a VT is executed, the VT is removed from this queue (see Figure 4.5).

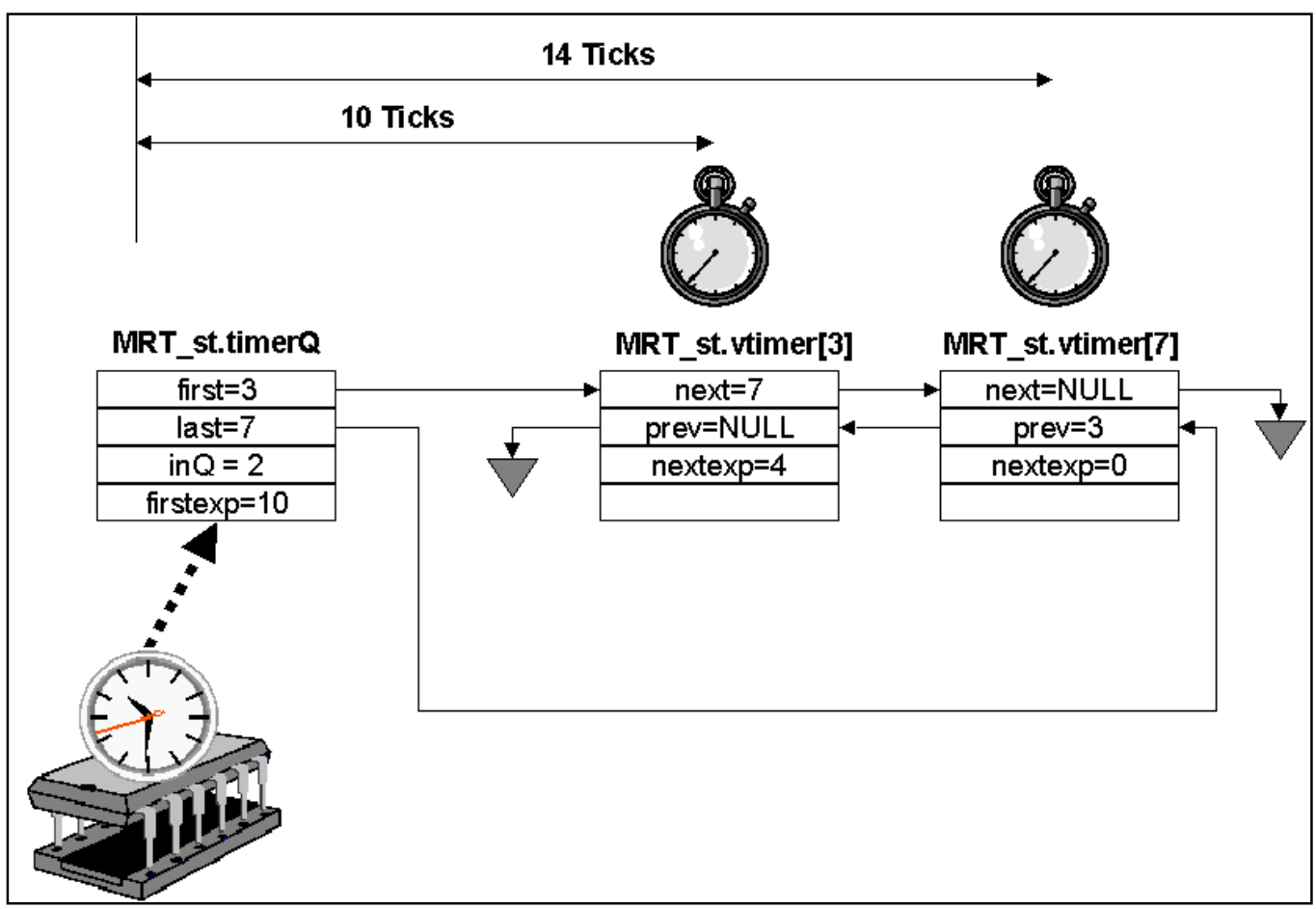

Figure 4.4: Active Virtual Timers Queue.

The data structure of a VT queue descriptors $\left(M R T \_\right.$timer $\left.Q \_t\right)$ has the following fields:

- first: A pointer to the first VT in the queue.

- last: A pointer to the last VT in the queue. 
- firstexp: It counts the number of RT-ticks to the first VT expiration.

- maxper: It is the total amount of nextexp fields of all VTs in the queue.

(only used in the Active queue).

- inQ: The current number of VTs enqueued.

- maxinQ: The maximun number of VTs enqueued.

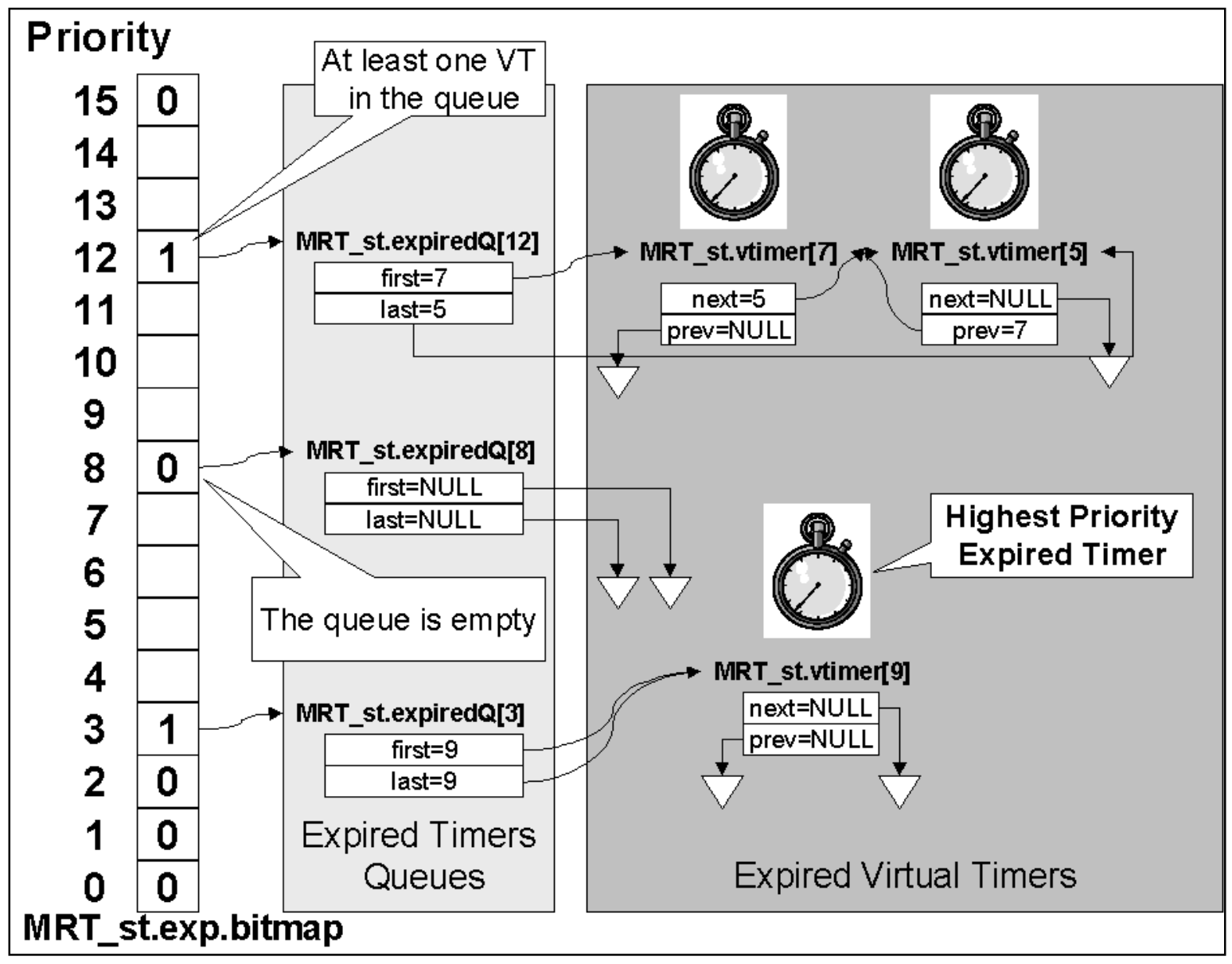

Figure 4.5: Virtual Timer Expired Queues.

\subsubsection{Executing Virtual Timers Actions}

Executing VT actions in the Timer handler could produce unpredictable latencies on high priority processes. As the Timer interrupt descriptor has the highest priority, all VT actions execute at the highest system priority including those actions of VTs that belongs to processes with lower priorities. This is another type of unbounded priority inversion. 
Executing VT actions in Bottom Halves routines does not help much, because the VT actions are executed before returning the system to user-mode, therefore the priority inversion persists.

As it is illustrated in Figure 4.6, the execution of actions of all expired VTs preempt the execution of a higher priority process.

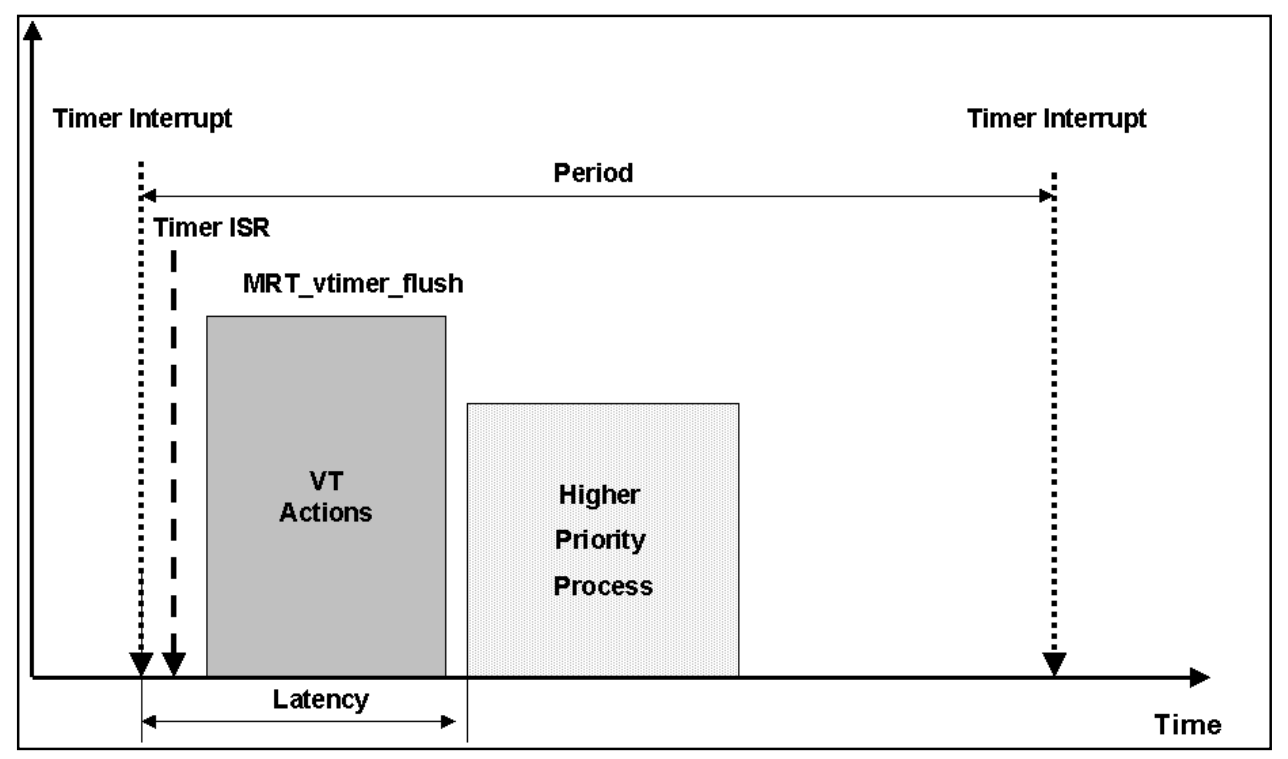

Figure 4.6: VT Actions with Unbounded Priority Inversion.

To avoid this problem, the RT-kernel runs expired VT actions in priority order only if they have higher or equal priorities than $M R T \_s v . p r t y l v l$ (see Figure 4.7).

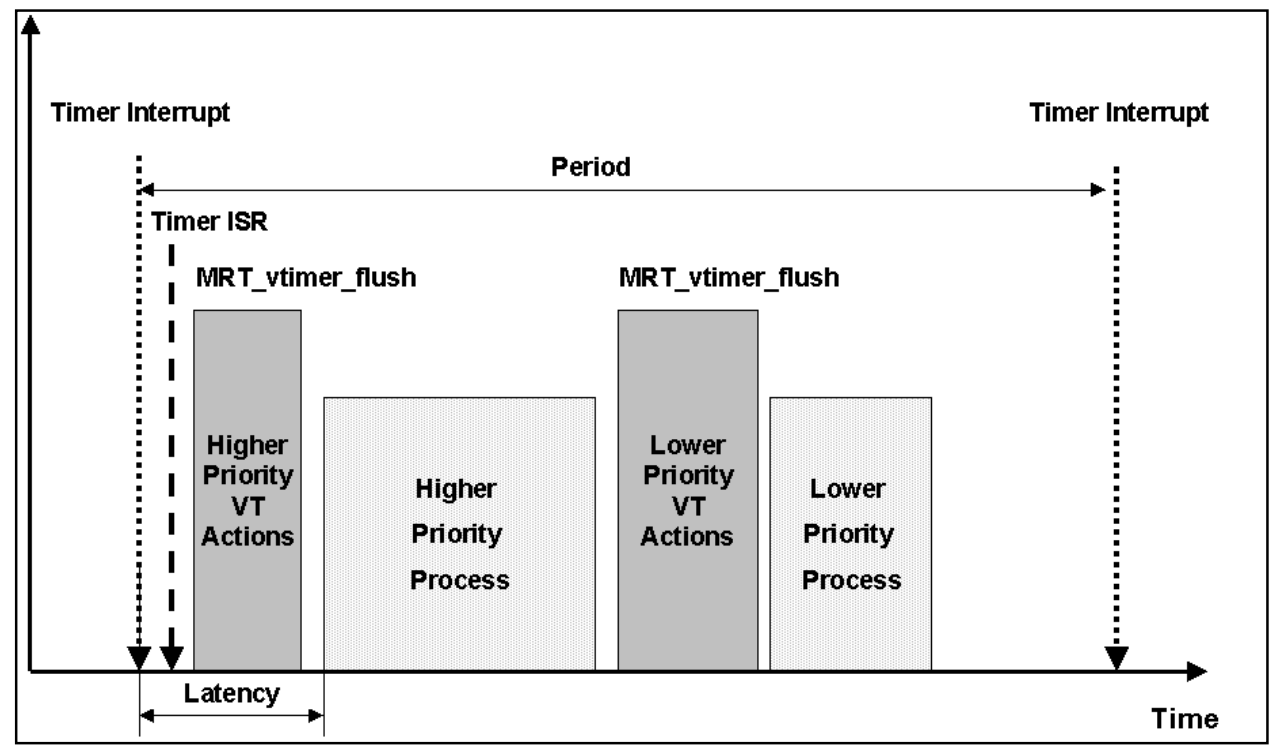

Figure 4.7: VT Actions Priority Ordered Execution. 
Only the execution of actions of all expired VTs with higher or equal priority than $M R T$ sv.prtylvl could preempt the execution of a running process or IRQ handler. VT actions with lower priorities will be executed later when the MRT_sv.prtylvl decline. The flow diagram is shown in Figure 4.8.

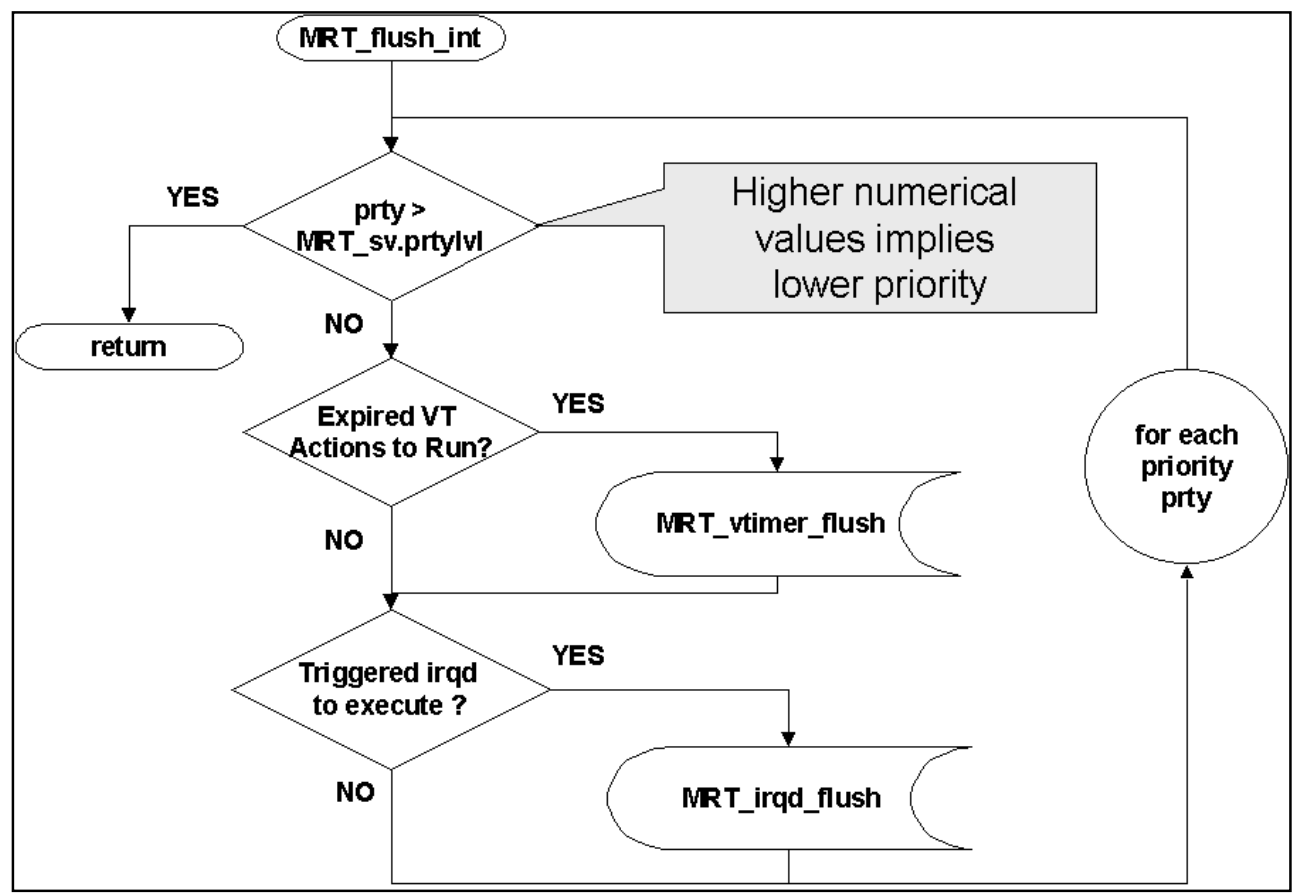

Figure 4.8: Flushing Expired VTs and Triggerd IRQ Descriptors.

\subsection{Virtual Timers Handling: Other Tested Approaches}

This section dicusses other approaches tested during the development and implementation of VTs on MINIX4RT.

Varghese and Lauck [34] suggest the use of Timer Wheels to handle a lots of VTs in an efficient way. This approach has been tested but the coding complexity, larger memory requirements and its minimal performance improvement on a reduced VT environment move further away the educational aims of the MINIX4RT project. In spite of that, that approach could be considered for custom versions or for coding laboratory practice.

When the PIT is used in SQUARE_WAVE mode, increasing Timer resolution implies increasing the PIT frequency rising the system overhead because the Timer ISR is executed more frequently. 
A better approach [35] to increase the Timer resolution without increasing the PIT frequency is to execute the Timer handler only in those moments that is needed. Instead of producing interrupts at higher rates (SQUARE_WAVE mode), the PIT is programmed in ONE-SHOT mode. This means that on every Timer interrupt, the PIT is programmed to generate an interrupt as soon as the earliest scheduled VT action. In spite of this feature is not available in the current version of MINIX4RT, it will be included in the next as an optional operation mode. 


\section{REAL-TIME INTERPROCESS COMUNICATION (RT-IPC)}

\subsection{Introduction}

The key difference between time sharing OS and RTOS is the need for deterministic timing behavior in the RTOS. Deterministic timing means that OS services consume only known and expected amounts of time. Inter-process communication (IPC) by message passing is one of the central paradigms of most kernel-based and other Client/Server architectures. It helps to increase modularity, extendibility, security and scalability, and it is the key feature for distributed systems and applications [흐]. Therefore, IPC primitives of a RTOS need to have deterministic execution and blocking times.

Messages are units of information that pass from the sender to the receiver. Two methods can be used to message transferences:

- With buffering: The message is sent to a data structure like RT-Mach ports[10] or Message Queues that stores it until a process receives it.

- Without buffering: The message is sent to a process and the sender must wait to tranfer the message. This method is known as rendezvous.

Tanenbaum selects the rendezvous approach for MINIX. It has the following semantics:

- When a sender calls send() but the receiver is not waiting that message, the sender is blocked until the receiver calls receive() for that message.

- When a receiver wants to receive a message but it has not been sent, the receiver is blocked until the sender calls send() for that message. 
Rendezvous approach is fine in a time-sharing environment because it is very rare that two or more messages are queued into a Message Queue and the avarage queue length would be less than one but it can not be used for asynchronous communications among processes.

MINIX's kernel hides interrupts turning them into messages, but interrupts are asynchronous events. When a I/O device raice an interrupt, its handler traps it, and will try to send() a message to an $\mathrm{I} / \mathrm{O}$ Task. If the I/O Task is not waiting for that message, the handler must register this fact and will try to send the message latter because the kernel can not be blocked. This approach does not help to much for the implementation of communications protocols where messages can flow from down to top triggered by interrupts.

In a RT-environment, several messages can be sent to a queue waiting to be received. They must be treated according the senders' priorities and must guarantee message delivery in a timely fashion[10].

MINIX4RT IPC uses unidirectional communication channels called Message Queues (MQ) consisting of a list that holds messages in kernel space. The number of messages that a MQ can store can be specified for each RT-process at creation time. Messages have fixed sizes and strict copy to value semantics.

\subsection{MINIX IPC Primitives}

MINIX has the following IPC kernel functions:

- mini_send(caller, dest, $m \_$ptr): A message is copied from the caller's message buffer pointed by $m \_$ptr to the dest's message buffer if dest process it is blocked waiting for that message, otherwise the caller process is blocked.

- mini_rec(caller, src, $\left.m \_p t r\right)$ : If the sender process $s r c$ is blocked trying to send a message to the caller process, the message is copied from the src's buffer to the buffer pointed by $m \_p t r$ and the $s r c$ process is unblocked, otherwise the caller process is blocked.

MINIX offers the following primitives in the libc.a library for higher layers: 
- send(dest, $m \_$ptr): A message is copied from the sender's message buffer pointed by $m_{\text {p }}$ tr to the dest's message buffer if dest process is blocked waiting for that message, otherwise the caller process is blocked.

- receive(src, $m$ p ptr): If the $s r c$ process is blocked trying to send a message to the receiver, the message is received and src process is unblocked, otherwise the receiver process is blocked.

- sendrec (dest_src, $m \_$ptr): A send() followed by a receive() to/from the same process in a single function. It is used for system calls avoiding to make two transitions from User-mode to Kernel-mode.

\subsection{MINIX4RT IPC Primitives Features}

Sometimes there are needs to use some type of policy to control the system behavior on message transfers. That policy may differ for messages sent to request services from those messages used by Servers to reply that requests. MINIX uses the same mini_send() primitive for both operations without distinguish among them.

The use of the same function for service requests, for service replies, to signal interrupts, etc. does not help for the apply a policy for each type of action.

A RT-process can not use MINIX IPC primitives because:

- mini_send() and mini_rec() kernel funcions could change the RTprocess status to READY, and therefore the RT-process would be selected to execute by MINIX scheduler loosing all its RT-execution attributes.

- As MINIX IPC does not support different behaviors for mini_send(), any priority inversion avoidance protocols can be applied.

- If a RT-process make a request to a a NRT-process using mini_send(), the RT-process must wait for the reply until NRT-process will run at NRTpriority. This is another case of Unbounded Priority Invesion (detailled in Section 5.17)

As a RT-process can not use MINIX IPC primitives, it is inhibited of making any MINIX System Calls (except exit() as was explained in Chapter 3). 
MINIX4RT offers a variety of new Kernel Calls that let apply different policies depending on the message transfer use. The term Kernel Calls is used to distinguish the way that they operate against System Calls. A more detailed explanation will be find in Chapter 6.

RT-IPC Kernel Calls have the following features:

- Synchronous/Asynchronous message transfer using Message Queues.

- Configurable Message Queue size.

- Different beahavior of send operations for requests, replies, signals and interrupts.

- Timeout support for synchronous primitives.

- Configurable dequeuing policy (Priority order or FIFO order).

- Priority Hand-Off to avoid unbounded priority inversion.

- Basic Priority Inheritance Protocol [37] support to avoid unbounded priority inversion.

- Sending timestamps and message IDs can be retrieved by the receiver.

- Senders process's attributes are stored with the message header (priority, process type, process deadline, etc).

The RT-kernel has a message pool named MRT_sm.pool that has NR_MESSAGES message queue entries in kernel space. Each message queue entry includes space for the message itself and its header.

When a NRT-process is prepared to be converted into a RT-process using the mrt_setproc() System Call a MQ is assigned to it. A MQ has a bounded capacity that quantifies its ability to store messages. The size requested of the MQ is a field of the mrt_pattr_t data structure passed as function parameter of $m r t$ setproc(). The $p m q$ field of the process descriptor is filled with a pointer to the message queue assigned to the process. 


\subsection{Message Descriptor Data Structure}

A Message Descriptor Data Structure ( $\left.m r t \_m s g d \_t\right)$ is composed by the Message Payload Data Structure and the Message Header Data Structure described in the next sections (see Appendix D).

\subsubsection{Message Payload Data Structure}

MINIX kernel defines six messages types (see Figure 5.1). The sizes of message elements will vary, depending upon the architecture of the machine; this diagram illustrates sizes on a machine with 32-bit pointers.

MINIX4RT keeps the message formats but without the first two fields ( $m$ _source and $m_{-}$type $)$in the Message Payload Data Structure (MRT_msg_t $)$.

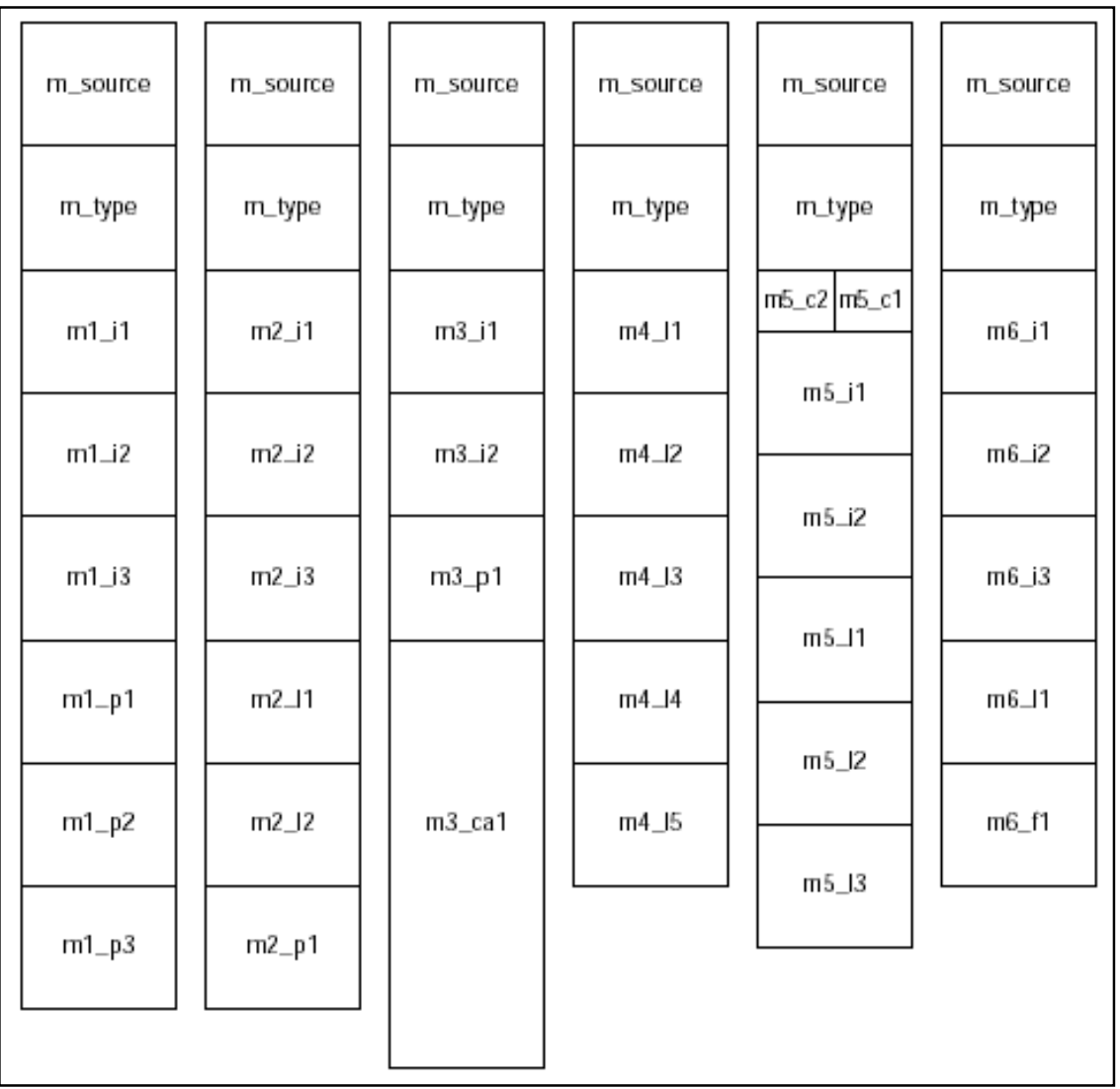

Figure 5.1: MINIX Message Types (From [2]). 


\subsubsection{Message Header Data Structure}

As the RT-kernel needs more information to describe a message, a Message Header Data Structure ( $\left.M R T_{-} m s g \_h d r_{-} t\right)$ with the following fields is defined:

- source: RT-PID of the process who sent the message.

- dest: RT-PID of the destination process.

- mtype: What kind of message is it.

- mid: A message ID set by the kernel.

- seqno: A message sequence number. It is the number of message sent by the sender RT-process.

- tstamp: The value of the system counter RTM_sv.counter.ticks when the message was inserted into the MQ.

- priority: The message priority that equals the sender's priority (see Section 5.18).

\subsection{The Message Queue Entry Descriptor}

A Message Queue Entry Descriptor (MQE) can store one Message Descriptor ( $m r t$ msgd_t $)$ and other fields that are needed to conform MQs and handling message timeouts. The fields of the $M R T \_m q e \_t$ data structures are (see Appendix D):

- msgd: A Message Descriptor.

- index: A Message Queue Entry ID.

- pvt: A pointer to a VT that handle the message timeouts.

- next: A forward pointer.

- prev: A backward pointer. 


\subsection{The Message Queue Descriptor}

A Message Queue Descriptor $\left(M R T \_m s g q \_t\right)$ let the RT-kernel organize MQEs. Each MQ descriptor have the following fields:

- index: The message queue ID used for quick searches.

- size: The message queue size.

- flags: The message queue policy flags:

- MRT_PRTYORDER: Priority Order Policy

- MRT_FIFOORDER: First In First Out Order Policy

- inQ: It counts the number of messages enqueued.

- $\operatorname{maxin} Q$ : It counts the highest number of message enqueued.

- owner: The message queue owner.

- delivered: The total number of messages delivered.

- enqueued: The total number of messages enqueued.

- $p v t$ : A pointer for the VT related with the MQ. It is used to handle the timeout of the mrt_rcv() Kernel Call.

- mQ: An array of queues to handle the messages in priority or FIFO order.

\subsection{The RT-System Message Pool}

The memory space where messages are stored is called the System Message Pool. It is allocated in kernel memory space before communications to eliminate the buffer allocation delay (see Figure 5.2).

The list that keeps the free Message Queue Entries is RTM_sm.mfreeQ. It has the same data structure type $M R T \_m s g q \_t$ than other MQs. 


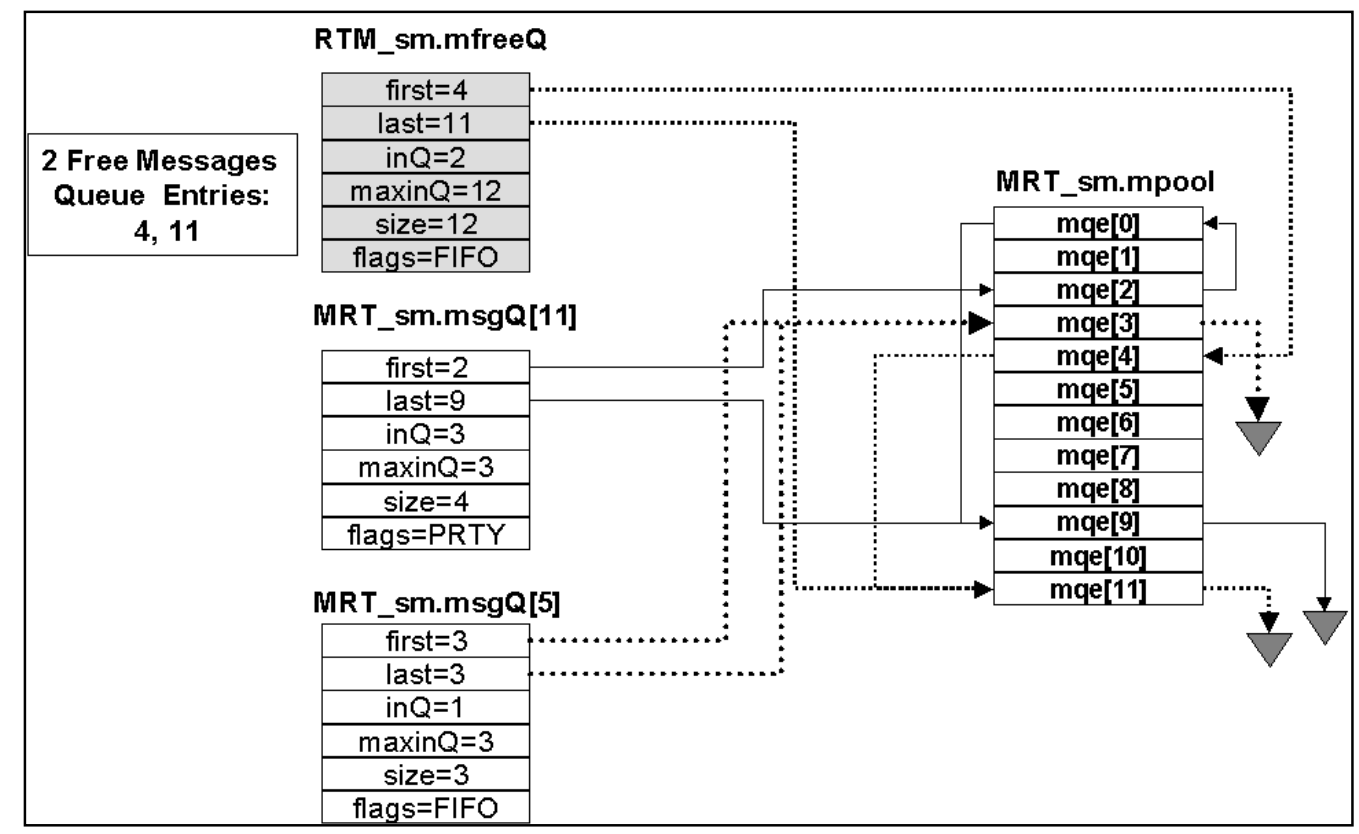

Figure 5.2: System Message Pool.

\subsection{Message Queues Management}

To manage the MQs the RT-kernel uses the $M R T \_m Q \_t$ data structure that is composed by:

A set of MQE queues, one queue assigned for each priority level.

A bitmap with one bit assigned for each priority. A bit set means that the related queue has at least one message. Initially, all the bits are cleared indicating that all queues are empty.

When a MQE is enqueued, the related bit to its priority is set in bitmap, and the MQE is appended to the queue in accordance with its priority field.

Finding the highest priority $\mathrm{MQE}$ in the $\mathrm{MQ}$ is therefore only a matter of finding the first bit set in bitmap. Because the number of priorities is fixed, the time to complete a search is constant and unaffected by the number of enqueued MQE in the queues.

Each queue have two pointers, one for the first message descriptor and one for the last message descriptor enqueued (see Figure 5.3). The insertions in the queue can be in FIFO or LIFO order. MQE of the same priority will be managed under a FIFO policy.

The following kernel functions help to manage MQs and MQEs:

- $\quad M R T \_m s g Q \_r s t():$ Resets all MQ fields. 
- MRT_msgQ_alloc(): Allocs a MQ for a RT-process.

- MRT_mqe_rst(): Resets all MQE fields.

- MRT_mqe_alloc(): Allocs a MQE from the Free queue for a MQ.

- MRT_mqe_free(): Releases a MQE returning it to the system Free queue.

- MRT_mqe_app(): Appends a MQE at the tail of a MQ.

- MRT_mqe_rmv(): Removes a MQE from a MQ.

- MRT_mqe_pick(): Selects the highest priority (Priority Ordering) or the oldest (FIFO Ordering) MQE sent by a specified source from a MQ.

As it can be seen, that the same design pattern was employed in the management of interrupt descriptor queues, ready process queues, expired timer queues and message queues.

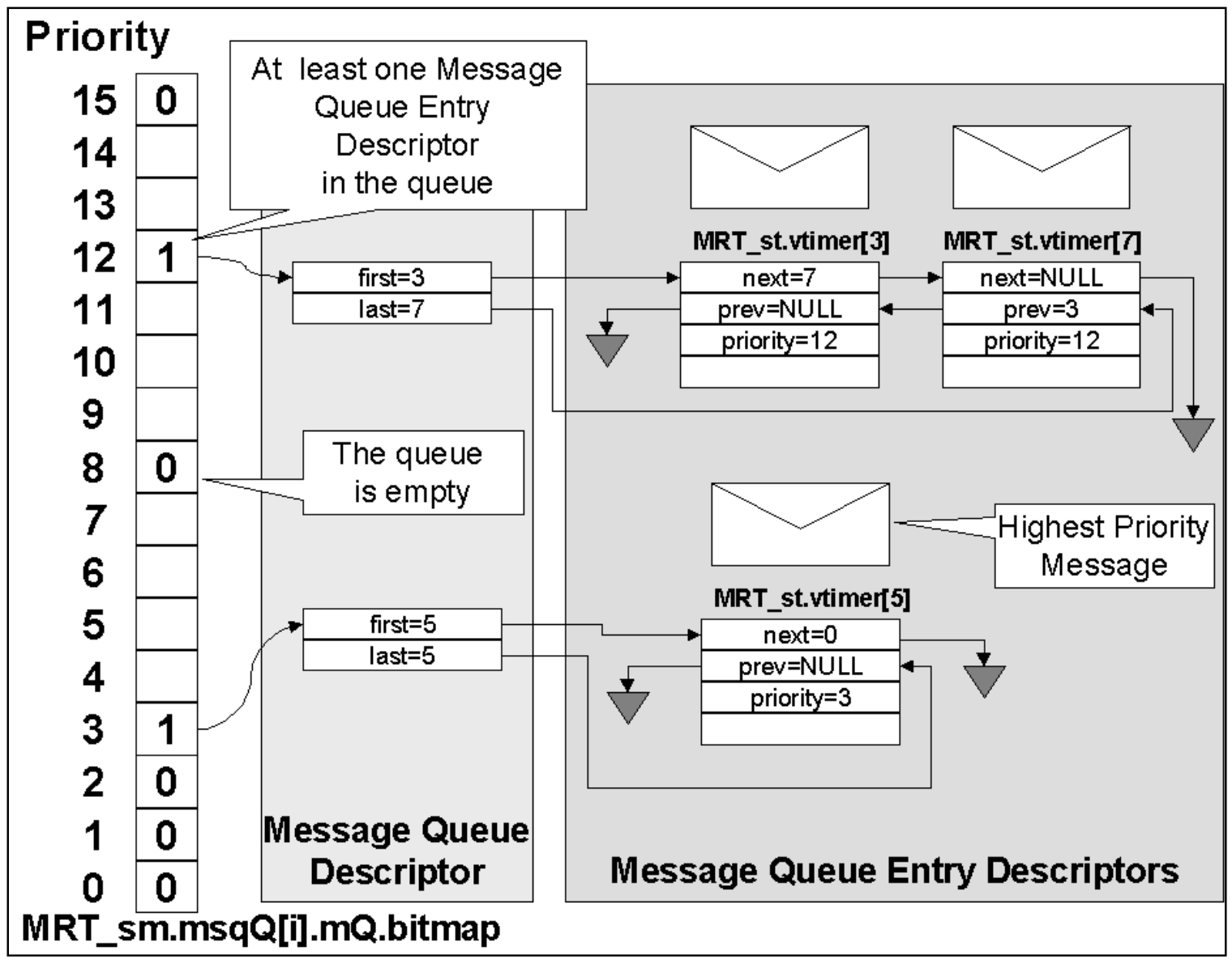

Figure 5.3: Message Queue Management. 


\title{
5.9. The mrt_rqstO Kernel Call
}

The mrt_rqst() Kernel Call sends a request message to a RT-process through a MQ in a synchronous manner specifying a timeout.

The Kernel Call prototype is:

\section{int mrt_rqst(dest, m_ptr, timeout)}

Where:

\author{
- dest. It is the RT-PID of the destination process. \\ - $\quad \boldsymbol{m} \_$ptr. It is a pointer to the message buffer. \\ - timeout. It is the time to wait that the request message could be sent.
}

If the dest proccess is waiting for the message, it is copied from the caller's message buffer pointed by $m$ ptr to the dest's process message buffer. The dest process inherits the caller's priority if it is higher than its owns.

If dest process is not waiting for the message, the request is enqueued in the MQ owned by the dest process, and the caller is blocked until the message is received. The dest process and all other processes requested directly and indirectly by the dest process inherit the caller's priority if it is higher than they own.

A timeout in Timer ticks can be specified to wait for the request message could be sent. A special value of MRT_NOWAIT can be specified to return without waiting if the dest process is not blocked receiving the message. To wait until the dest process will receive the message, MRT_FOREVER must be specified as a timeout. If the timeout expired:

- The message is removed from the dest's MQ.

- The caller process is unblocked returning and E_MRT_TIMEOUT error code.

- The dest's process priority is set to the highest priority message in it's MQ. 


\title{
5.10. The mrt_arqst() Kernel Call
}

The mrt_arqst() Kernel Call sends a request message to a process through a MQ in an asynchronous manner.

The Kernel Call prototype is:

\section{int mrt_arqst(dest, m_ptr)}

Where:

\author{
- $\quad$ dest. is the RT-PID of the destination process. \\ - $\quad$ m_ptr: is a pointer to the message buffer.
}

If the dest proccess is waiting for the message, it is copied from the caller's message buffer pointed by $m \_$ptr to the dest's process message buffer. The dest process inherits the caller's priority if it is higher than its owns.

If dest process is not waiting for the message, the request is enqueued in the dest's MQ, and the caller returns without waiting for the message will be received. The dest process and all other processes requested directly and indirectly by the dest process inherit the caller's priority if it is higher than they own.

\subsection{The mrt_replyO Kernel Call}

The mrt_reply() Kernel Call sends a message to a process through a MQ in an asynchronous manner. It can be used for replies in a botton-up way (i.e. Server to Client).

The Kernel Call prototype is:

int MRT_reply(dest, m_ptr)

Where:

- dest. is the RT-PID of the destination process.

- $\boldsymbol{m} \_$ptr: is a pointer to the message buffer. 
If the dest process is blocked waiting for the reply, the message is copied from the caller's memory $m \_$tr to the dest process message buffer and dest is unblocked.

If the dest process is not blocked waiting for the message, the reply is enqueued in the dest process MQ.

At last, the caller's priority is set to the highest priority message in it's MQ or to its base priority baseprty if its MQ is empty.

\subsection{The mrt_uprqst $O$ Kernel Call}

The mrt_uprqst() Kernel Call sends a message to a process through a MQ in an asynchronous manner. It can be used by the Tasks to make requests coming from remote processes in a botton-up way.

The Kernel Call prototype is:

\section{int mrt_uprqst(dest, m_ptr, priority)}

Where:

- dest. is the RT-PID of the destination process.

- $\boldsymbol{m} \_$ptr: is a pointer to the message buffer.

- priority. is the priority of the message.

If the dest proccess is waiting for the message, it is copied from the caller's message buffer $m \_$tr to dest's process message buffer.

If the dest process is not waiting the message, it is enqueued into the dest process MQ.

In both cases, the priority of the dest process will be changed if and only if its current priority is lower than the specified priority function argument.

\subsection{The mrt_signO Kernel Call}

The mrt_sign() Kernel Call sends a message to a process through a MQ in an asynchronous manner. It can be used by the Tasks to sign processes in a botton-up way. 
The Kernel Call prototype is:

int mrt_sign(dest, m_ptr)

Where:

- dest. is the RT-PID of the destination process

- $\boldsymbol{m} \_$ptr: is a pointer to the message buffer

If the dest process is blocked waiting for the message, it is copied from the caller's memory $m \_$ptr to the dest's process message buffer.

If the dest process is not waiting the message, the message is enqueued into the dest's process MQ.

\subsection{The MRT_send $O$ Kernel Function}

The MRT_send() Kernel Function sends a message to a process through a MQ in asynchronous manner. It can be used by ISRs to send messages to Tasks in a botton-up way.

The Kernel Call prototype is:

\section{int MRT_send(dest, prty, mtype)}

Where:

- dest. The RT-PID of the destination process.

- prty. The message priority.

- mtype: The message type.

If the dest process is blocked waiting for the message, the message header is copied into the dest's process message buffer.

If the dest process is not blocked waiting the message, the message header is copied into the dest's process MQ, the field st.mdl (Missed DeadLines) of the dest process's descriptor is increased and a MT_SIGNAL message is sent to the watchdog process of the dest process. 


\subsection{The mrt_rcvO kernel Call}

The mrt_rcv() Kernel Call is used to receive a message.

The Kernel Call prototype is:

int mrt_rcv(source, hdr_ptr, m_ptr, timeout)

Where:

- source: specify the RT-PID of the message sender from which the caller wants to receive a message. A special value of $M R T_{-} A N Y P R O C$ can be specified to receive a message from any source.

- hdr_ptr: specify the caller's buffer for the message header.

- $\quad \boldsymbol{m} \_$ptr: specify the caller's buffer for the message payload.

- timeout. specify a timeout to wait for the message. Two special values can be specified:

- MRT_NOWAIT: The process returns with o without receiving the message. If the message has been received, the function return code is $O K$ else returns $E_{-} T R Y \_A G A I N$.

- MRT_FOREVER: The process waits until the message is receive.

The caller searches for a message from the specified source into it's MQ with the retrieving policy of the MQ (Priority or FIFO order).

If there are no message from the source process, the caller is blocked.

If the source process is blocked trying to send the message in a synchronous manner it is unblocked.

The caller process can specify a timeout to unblock itself. If no message from the specified source is received in the specified period it returns $E_{-} M R T \_T I M E O U T$ error code.

If a message is received, the $h d r \_$ptr buffer is filled with the header fields of the message received. 


\subsection{The mrt_rqrevO kernel Call}

The mrt_rqrcv() Kernel Call optimize the performance of the common operations of send a request message to a server process and waits for the reply message. It saves one context switch for the caller process.

The Kernel Call prototype is:

\section{int mrt_rqrev(dest, rqst, rply, hdr, timeout)}

Where:

- dest. Specifies the RT-PID of the destination processs.

- rqst. Specifies the caller's buffer for the request message.

- $\quad$ rply. Specifies the caller's buffer for the reply message payload.

- $\quad \boldsymbol{h d r}$. Specifies the caller's buffer for the message header.

- timeout. specify a timeout to wait for the message. A special value of MRT_FOREVER can be specified to wait until the reply message is received.

\subsection{Using MINIX4RT IPC Kernel Calls}

Table 5.1 resumes the uses of send-type RT-Kernel Calls. Table 5.2 resumes the uses of the mrt_rcv() RT-Kernel Call.

Table 5.1: Send-type Kernel Calls Uses.

\begin{tabular}{|c|l|l|l|l|}
\hline Use & Kernel Call & \multicolumn{1}{|c|}{$\begin{array}{c}\text { Caller's Process } \\
\text { Priority }\end{array}$} & $\begin{array}{l}\text { Destination's Process } \\
\text { Priority }\end{array}$ & Caller waits until \\
\hline Synchronous Request & $m r t$ rqst() & It is not changed & $\begin{array}{l}\text { It is set to the caller's } \\
\text { priority if it higher } \\
\text { than it's current } \\
\text { priority }\end{array}$ & $\begin{array}{l}\text { The message is received or a } \\
\text { timeout expire. }\end{array}$ \\
\hline $\begin{array}{c}\text { Asynchronous Request } \\
\text { (used by non blocking } \\
\text { Servers ) }\end{array}$ & mrtarqst() & It is not changed & $\begin{array}{l}\text { It is set to caller's It does not wait } \\
\text { priority if it higher } \\
\text { than it's current } \\
\text { priority }\end{array}$ \\
\hline
\end{tabular}


Table 5.1: Send-type Kernel Calls Uses (cont.)

\begin{tabular}{|c|c|c|c|c|}
\hline Use & Kernel Call & $\begin{array}{c}\text { Caller's Process } \\
\text { Priority }\end{array}$ & $\begin{array}{c}\text { Destination's Process } \\
\text { Priority }\end{array}$ & Caller waits until \\
\hline Reply & mrt_replyo & $\begin{array}{l}\text { It is set to the value } \\
\text { of the highest } \\
\text { priority message in } \\
\text { its MQ or to its base } \\
\text { priority }\end{array}$ & It is not changed & It does not wait \\
\hline Up request message & mrt_uprqst() & It is not changed & $\begin{array}{l}\text { It is set to speficied } \\
\text { priority if it is higher } \\
\text { than its current priority }\end{array}$ & It does not wait \\
\hline Signal message & mrt_sign() & It is not changed & It is not changed & It does not wait \\
\hline Request \& Receive & mrt_rqrcv() & It is not changed & $\begin{array}{l}\text { It is set to the caller's } \\
\text { priority if it is higher } \\
\text { than it's current } \\
\text { priority }\end{array}$ & $\begin{array}{l}\text { The reply message is received } \\
\text { or a timeout expire. }\end{array}$ \\
\hline $\begin{array}{l}\text { Send a message } \\
\text { (used by ISR) }\end{array}$ & $M R T \_$send () & It is not changed & $\begin{array}{l}\text { It is set to speficied } \\
\text { priority if it is higher } \\
\text { than its current priority }\end{array}$ & $\begin{array}{l}\text { It does not wait if the } \\
\text { destination process is waiting } \\
\text { the message or enqueue the } \\
\text { message and increase de } \\
\text { missed deadline of the } \\
\text { destination process }\end{array}$ \\
\hline
\end{tabular}

Table 5.2: mrt_rcvO Kernel Calls Uses.

\begin{tabular}{|c|c|c|}
\hline mrt_rcv use & source & timeout \\
\hline To receive a reply. & RT-PID of the requested process & MRT_NOWAIT $<$ timeout $<$ MRT_FOREVER \\
\hline To receive requests. & MRT_ANYSRC & MRT_FOREVER \\
\hline
\end{tabular}

\subsection{Priority Invertion}

In many RT-applications, there are resources that must be shared among processes in a way that prevents more than one process from using the resource at any moment (mutual exclusion). Priority inversion is the term used to describe a situation where a process is waiting for a lower priority process to free a shared, exclusive use resource. Clearly, a priority based RT-system cannot tolerate significant periods of priority inversion. A sample helps to illustrate the problem. See Figure 5.4, where:

$$
\boldsymbol{T}_{\boldsymbol{H}} \text {. Highest Priority Process. }
$$


$\boldsymbol{T}_{\boldsymbol{M}}$ : Medium Priority Process.

$\boldsymbol{T}_{\boldsymbol{L}}$ : Lowest Priority Process.

$\boldsymbol{S}$ : Server Process.

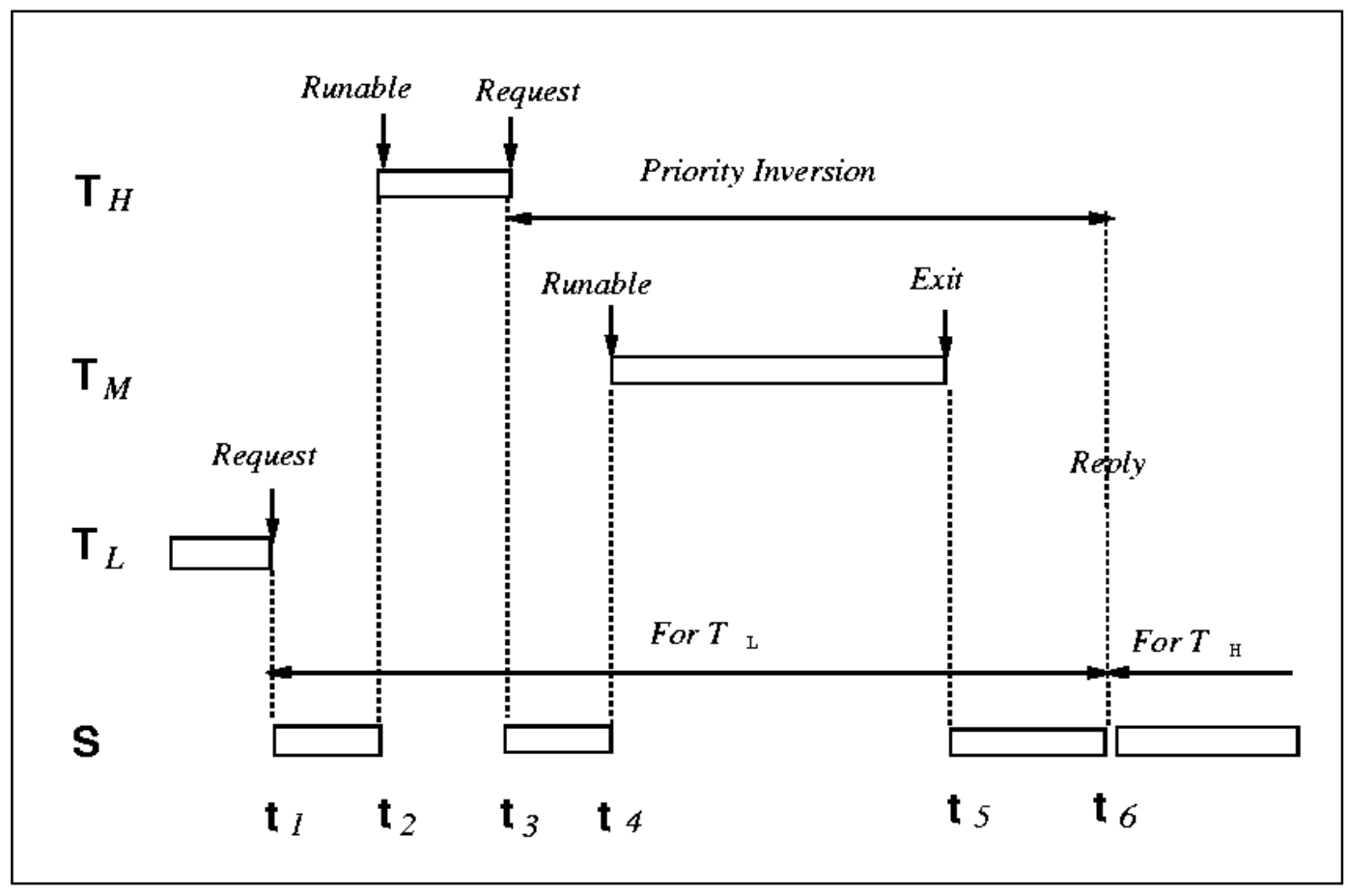

Figure 5.4: Priority Inversion.

The sequence of events are:

$t_{1}: T_{L}$ requests a service to $S$.

$\boldsymbol{t}_{2:} \boldsymbol{S}$ is preempted by $\boldsymbol{T}_{\boldsymbol{H}}$.

$\boldsymbol{t}_{3:} \boldsymbol{T}_{\boldsymbol{H}}$ requests a service to $\boldsymbol{S}$.

$\boldsymbol{t}_{4}$. $\boldsymbol{S}$ stills working on $\boldsymbol{T}_{L}$ request.

$\boldsymbol{t}_{\mathbf{5}}: \boldsymbol{T}_{\boldsymbol{M}}$ preempts $\boldsymbol{S}$.

$\boldsymbol{t}_{\boldsymbol{\sigma}}$. $\boldsymbol{T}_{\boldsymbol{M}}$ exits and $\boldsymbol{S}$ stills working on $\boldsymbol{T}_{\boldsymbol{L}}$ request.

$\boldsymbol{t}_{7}$ S $\boldsymbol{S}$ replies to $\boldsymbol{T}_{L}$ and starts working on $\boldsymbol{T}_{\boldsymbol{H}}$ request. 
The time interval between $\boldsymbol{t}_{\mathbf{3}}$ and $\boldsymbol{t}_{\boldsymbol{6}}$ is a Priority Inversion, but the interval between $\boldsymbol{t}_{\mathbf{4}}$ and $\boldsymbol{t}_{\mathbf{5}}$ may be unbounded.

The unbounded priority inversion is one serious problem in RT-systems. There has been developed many mechanisms to avoid it. The priority inversion problem in Client/Server communication is more serious one, since the length of priority inversion tends to be much longer than that of synchronization.

\subsection{Basic Priority Inheritance Protocol (BPIP)}

Sha, Rajkumar and Lehosky [료 have proposed two protocols to avoid the priority inversion problem. One is the Basic Priority Inheritance Protocol (BPIP), the other is the Priority Ceiling Protocol (PCP).

Priority inheritance mechanisms are intended to prevent unbounded priority inversion by adjusting the effective priority of a lower priority process $\boldsymbol{T}_{\boldsymbol{L}}$ whenever a higher priority process $\boldsymbol{T}_{\boldsymbol{H}}$ is suspended waiting to be served.

A Server process $\boldsymbol{S}$ is said to be executing on behalf of a Client process $\boldsymbol{P}$ if $\boldsymbol{S}$ is executing a $\boldsymbol{P}_{\mathrm{S}}$ request or another process request that is executing a $\boldsymbol{P}_{\mathrm{S}}$ request.

A process $\boldsymbol{S}$ is said to be blocking process $\boldsymbol{P}$ if $\boldsymbol{P}$ (or a Server executing on behalf of $\boldsymbol{P}$ ) has made a request to $\boldsymbol{S}$, but $\boldsymbol{S}$ is executing other process request.

The BPIP consists of the following rules [37]:

- A Server process $\boldsymbol{S}$ executes at its runtime assigned priority when it does not block any process or when it is not executing on behalf of a Client.

- If a Server process $\boldsymbol{S}$ is executing on behalf of a Client process $\boldsymbol{P}$ or is blocking one or more process, $\boldsymbol{S}$ executes at either the priority of $\boldsymbol{P}$ or the priority of the highest priority process (if any) that $\boldsymbol{S}$ blocks, whichever is higher.

- Process requesting services of other process are served in priority order.

Under the BPIP, a Client process can be blocked in two ways: 
- Directly: when the called process either has a queued request or is executing other process request.

- Indirectly or push-through: when a process inherits a higher priority.

The BPIP potentially requires priorities to be modified when processes make requests. A Server process may inherit the priority of a higher priority Client process even though the Server is not executing that Client request. Thus, each Server has an assigned base priority (the baseprty field) and an effective priority (the priority field).

The behavior of BPIP in a Client/Server application is illustrated in Figure 5.5.

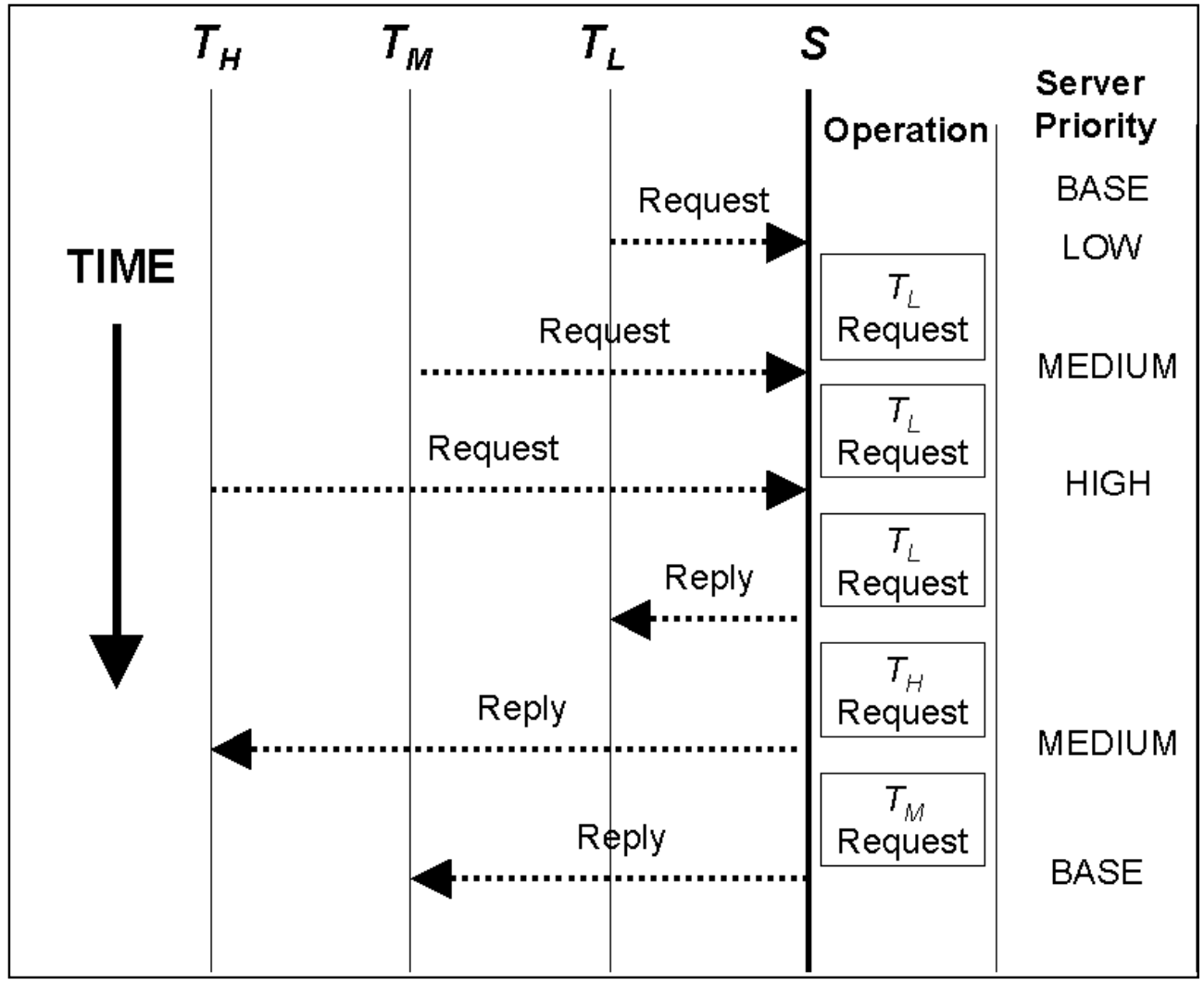

Figure 5.5: Basic Priority Inheritance Protocol Sample.

Asumptions:

- $\quad H I G H, M E D I U M, L O W, B A S E$ are process priorities.

- $\quad H I G H>M E D I U M>L O W>B A S E$.

- $\quad$ S's priority $=B A S E$.

- $\quad \boldsymbol{T}_{\boldsymbol{L}}$ 's priority $=L O W$. 
- $\quad \boldsymbol{T}_{\boldsymbol{M}}{ }^{\prime}$ s priority $=$ MEDIUM.

- $\quad \boldsymbol{T}_{\boldsymbol{H}}$ 's priority $=H I G H$.

- $\quad \boldsymbol{T}_{\boldsymbol{L}}$ priority $=L O W$

The sequence of events is described below:

- $\boldsymbol{T}_{\boldsymbol{L}}$ sends a request message to $\boldsymbol{S}$.

- $\quad \boldsymbol{S}$ executes on behalf of $\boldsymbol{T}_{\boldsymbol{L}}$ with $\boldsymbol{T}_{\boldsymbol{L}}$ 's priority.

- While $\boldsymbol{S}$ is processing $\boldsymbol{T}_{\boldsymbol{L}}$ 's request, $\boldsymbol{T}_{\boldsymbol{M}}$ preempts $\boldsymbol{S}$ and sends a new request message to it. As $\boldsymbol{S}$ has not finished with $\boldsymbol{T}_{\boldsymbol{L}}$ 's request, it is still running but at $\boldsymbol{T}_{\boldsymbol{M}}$ 's priority because it is blocking $\boldsymbol{T}_{\boldsymbol{M}}$.

- While $\boldsymbol{S}$ is processing $\boldsymbol{T}_{\boldsymbol{L}}$ 's request, $\boldsymbol{T}_{\boldsymbol{H}}$ preempts $\boldsymbol{S}$ and sends a new request message to it. As $\boldsymbol{S}$ has not finished with $\boldsymbol{T}_{\boldsymbol{L}}$ 's request, it is still running but at $\boldsymbol{T}_{\boldsymbol{H}}$ 's priority because it is blocking $\boldsymbol{T}_{\boldsymbol{H}}$.

- Afterward $\boldsymbol{S}$ finishs with $\boldsymbol{T}_{\boldsymbol{L}}$ 's request and sends a reply message to $\boldsymbol{T}_{\boldsymbol{L}}$ 's. Then, it gets the highest priority message from it MQ ( $\boldsymbol{T}_{\boldsymbol{H}}$ 's request), and starts to process it.

- When $\boldsymbol{S}$ finishs with $\boldsymbol{T}_{\boldsymbol{H}}$ 's request, it sends a reply message to $\boldsymbol{T}_{\boldsymbol{H}}$ and it's priority is changed to the highest priority message from it MQ ( $\boldsymbol{T}_{\boldsymbol{M}}$ 's request) waiting to be received.

- When $\boldsymbol{S}$ finishs with $\mathrm{T}_{\mathrm{M}}$ 's request, it sends a reply message to $\boldsymbol{T}_{\boldsymbol{M}}$ and it's priority is changed to the highest priority message waiting to be received but as the MQ is empty, it's efective priority is reset it's base priority.

In the previous sample, the higher priority process $\boldsymbol{T}_{\boldsymbol{H}}$ must wait that $\boldsymbol{S}$ finishs with the lower priority process $\left(\boldsymbol{T}_{\boldsymbol{L}}\right)$ request. It is a bounded priority inversion limited to the time consumed by $\boldsymbol{S}$ when it was executing on behalf of $\boldsymbol{T}_{\boldsymbol{L}}$.

To achieve the correct behavior and be compliance with BPIP, priority inheritance needs to be a transitive operation. Therefore, the RT-kernel must search across the chain of requested processes to apply the priority inheritance until it finds the process that has no pending requests. To search across the chain of a requested process, the RT-kernel uses mrt_sendto and mrt_getfrom fields of process descriptors. 


\subsection{The Priority Ceiling Protocol (PCP)}

One drawback of the BPIP is that it not prevents deadlocks and chained blocking. Dutertre [39] shows this behaviour on a BPIP implementation based on semaphores. MINIX4RT is a systen based on message transfer and its IPC primitives are based on the proposal of Borger and Rajkumar [37].

The kinds of deadlocks exposed by Dutertre can not occur in MINIX4RT top-down Client/Server programming model because all request goes down and replies goes up. It has the same behavior that imposing a total ordering on resource use. System programmers must consider this programming model to avoid deadlocks.

A necessary conditions for deadlocks is the use of blocking primitives, but MINIX4RT offers blocking and non-blocking Kernel calls with and without timeouts.

Chained blocking occurs when the system uses semaphores and a higher priority process $\boldsymbol{T}_{\boldsymbol{H}}$ is blocked by several processes of lower priorities in succession. For example, a process $\boldsymbol{T}_{\boldsymbol{M}}$ locks a semaphore $\boldsymbol{S}_{1}$ and a process $\boldsymbol{T}_{\boldsymbol{L}}$ locks a semaphore $\boldsymbol{S}_{2}$. When $\boldsymbol{T}_{\boldsymbol{H}}$ tries to lock $\boldsymbol{S}_{\boldsymbol{1}}$ it fails and $\boldsymbol{T}_{\boldsymbol{M}}$ inherits $\boldsymbol{T}_{\boldsymbol{H}}$ 's priority. When $\boldsymbol{T}_{\boldsymbol{M}}$ tries to lock $\boldsymbol{S}_{2}$ it fails and $\boldsymbol{T}_{\boldsymbol{L}}$ inherits $\boldsymbol{T}_{\boldsymbol{M}}$ 's priority. $\boldsymbol{T}_{\boldsymbol{H}}$ must wait until $\boldsymbol{T}_{\boldsymbol{L}}$ release $\boldsymbol{S}_{2}$ and $\boldsymbol{T}_{\boldsymbol{M}}$ releases $\boldsymbol{S}_{\boldsymbol{1}}$ although $\boldsymbol{T}_{\boldsymbol{H}}$ does not use $\boldsymbol{S}_{2}$ directly.

Chained blocking could occurs in a system with message transfers as MINIX4RT. For example, a process $\boldsymbol{T}_{\boldsymbol{M}}$ sends a request message to Server $\boldsymbol{S}_{\boldsymbol{1}}$ and a process $\boldsymbol{T}_{\boldsymbol{L}}$ sends a request message to Server $\boldsymbol{S}_{2}$. If $\boldsymbol{T}_{\boldsymbol{H}}$ sends a request message to Server $\boldsymbol{S}_{\boldsymbol{1}}$, it must wait and $\boldsymbol{S}_{\boldsymbol{1}}$ inherits $\boldsymbol{T}_{\boldsymbol{H}}$ 's priority. If $\boldsymbol{S}_{\boldsymbol{1}}$ sends a request message to Server $\boldsymbol{S}_{2}$ to process $\boldsymbol{T}_{\boldsymbol{M}}{ }^{\prime}$ s request, it must wait and $\boldsymbol{S}_{2}$ inherit $\boldsymbol{T}_{\boldsymbol{H}}{ }^{\prime} \mathrm{S}$ priority (transitive). $\boldsymbol{T}_{\boldsymbol{H}}$ is blocked waiting for:

- The reply of $\boldsymbol{S}_{2}$ to $\boldsymbol{T}_{\boldsymbol{L}}$.

- The reply of $\boldsymbol{S}_{2}$ to and $\boldsymbol{S}_{1}$.

- The reply of $\boldsymbol{S}_{1}$ to $\boldsymbol{T}_{\boldsymbol{M}}$.

- The reply of $\boldsymbol{S}_{\boldsymbol{I}}$ to $\boldsymbol{T}_{\boldsymbol{H}}$.

If PCP would be developed for MINIX4RT, it will need to set a priority ceiling for each process. As Server and Task processes could be requested by any User-level processes, the priority ceiling of all Servers and Tasks must be set to the highest User-level process priority. If a process request a service and the Server is blocked waiting a synchronous I/O, any other User-level process that requests another non related service to other Server will be blocked, because the Priority Ceiling 
is equal to the highest priority, disabling that any other request be treated until the former will finish. Therefore, the systems has the same behaviour than a monolitic non reentrant kernel.

If Server's Priority Ceiling is greater than or equal to any other User-level processes's priority, the use of PCP slows down the system performance because only one process could be attended by the RTOS at once.

\subsection{Complete Priority Inheritance}

Both, the BPIP and the PCP use the priority inheritance as a method to avoid the unbounded priority inversion problem. Those protocols require process priorities to be modified, but changing the priority of a process if one of the operations needed to implement priority inheritance. A RT-process has resources as VTs and messages sent to other processes that are handled according to their priorities, therefore they must change their priorities too.

To achieve a correct behavior of IPC primitives a Complete Priority Inheritance needs the following actions on processes and resources:

- If the process is in the RT-ready state, it must be removed from its current RT-ready Queue and inserted it into other RT-ready Queue according to its inherit priority.

- All process's expired VTs must be removed from their current Expired VT Queues and inserted into other Expired VT Queues according to the inherit priority of their owner.

- All messages sent to other processes must be removed from their current MQE lists and inserted into other MQE list according to the inherit priority of the sender.

If a request message sent by a lower priority process $\boldsymbol{T}_{\boldsymbol{L}}$ does not change its priority when $\boldsymbol{T}_{\boldsymbol{L}}$ inherits a higher priority, $\boldsymbol{T}_{\boldsymbol{L}}$ request may be (unbounded) delayed by other medium priority requests sent to the same destination process. Therefore a Client with higher priority that request a service from $\boldsymbol{T}_{\boldsymbol{L}}$ will be delayed too.

As it can be seen, the Complete Priority Inheritance could implies a performance penalty to IPC and increases the inversion delays produced by the OS itself [27]. The current version of MINIX4RT only changes the priority of the first process in the inheritance chain. 


\section{RT-SYSTEM CALLS, KERNEL CALLS AND FUNCTIONS}

\subsection{MINIX System Calls Implementation}

MINIX offers mini_send() and mini_rec() basic kernel primitives as was explained in Chapter 5. At higher levels send(), receive() and sendrec() are used. All System Calls are implemented using sendrec() to send the request to a server process and to wait the reply from it. The standard servers are Memory Manager $(M M)$ and File Sytem manager $(F S)$. These interactions are deeply explained by Ashton []ㅡ.

An example illustrates the implementation of a MINIX System Call (see Figure6.1).

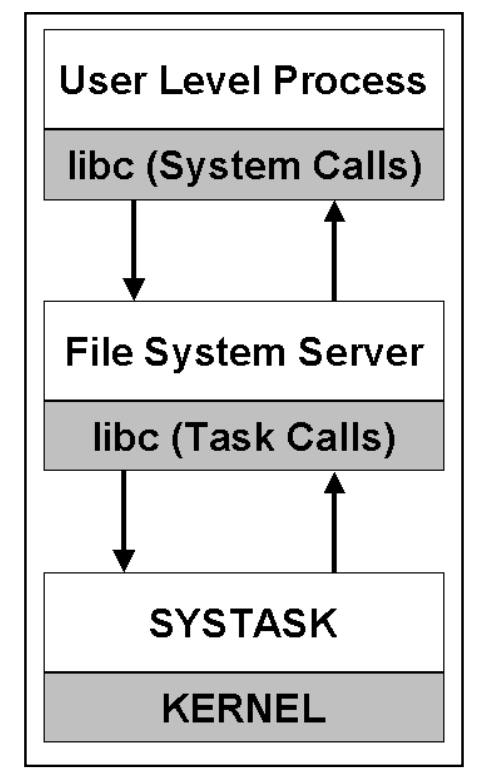

Figure 6.1: MINIX System Calls Implementation.

When a User-level process wants to get its user time consumed it calls utime(), the call is converted by the system library (libc.a) into a sendrec() Call to the FS server. As the $F S$ server does not have the information requested, it must transfer the request to a Task named SYSTASK using a 
Task Call (with_taskcall() function). A Task Call is like a System Call used by servers to request services to Tasks. Task Calls use send()/receive() primitives too.

As Tasks share the kernel memory space and data structures, the SYSTASK is like a kernel representative that can get process accounting times from kernel tables to reply to the $F S$ server that can reply to the User-level process.

\subsection{MINIX4RT System Calls Implementation}

MINIX4RT offers a set of System Calls that operates in a similar way as MINIX System Calls does. Unlike MINIX, it does not use the SYSTASK for source code readability.

For those time constrained services MINIX4RT offers Kernel Calls that are not inplemented using message transfers. They call RT-kernel services through a processor Trap.

The following sections describe how MINIX4RT implements System Calls and Kernel Calls and Appendix D is the reference of data structures used by them.

\subsubsection{RT-System Calls with Message Transfers}

Some RT-services do not need be requested by RT-processes. Examples of these services are the start of the Real-Time Processing Mode, stops it, gets system statistics, etc. They are not used for time constrained functions and they use MINIX send()/receive() primitives. Therefore, the RT-kernel could preempt the requesting process during the Sytem Call if a RT-process is ready to run. These type of RT-related System Calls are implemented using the MM server that interacts with a new task called MRTTASK (see Figure 6.2)

MRTTASK is like a RT-kernel representative that can access to RT-kernel data structures to reply to the $M M$ server that can reply to the User-level process. 


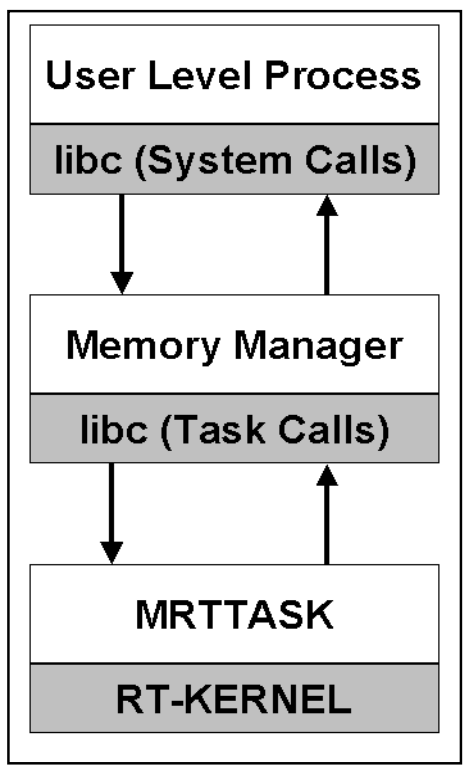

Figure 6.2: RT-System Calls with Message Transfers.

\subsubsection{RT-Kernel Calls without Message Transfers}

For time constrained services the MINIX4RT offers RT-kernel Calls. They interact with the RT-kernel directly without message transfers as in a monolitic OS using a processor Trap. (see Figure 6.3).

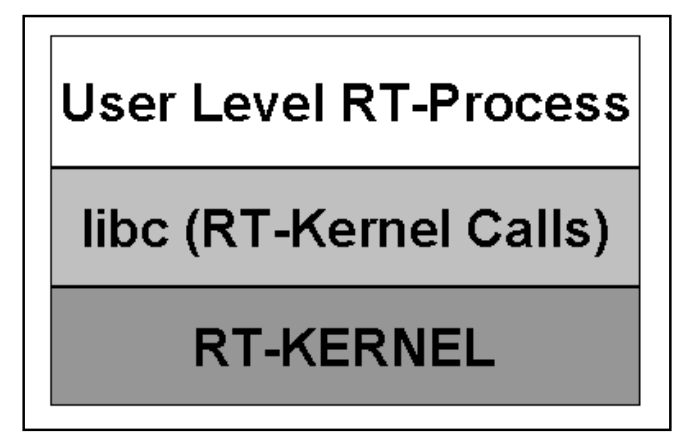

Figure 6.3: RT-Kernel Calls without Message Transfers.

This approach does not generate a sequence of context switches but implies a change from User-mode to Kernel-mode and viceversa.

\subsubsection{The RT-PID}

Every process in the system has an assigned unique identifier named the process identifier (PID). PIDs are the common mechanism used by applications to reference 
processes. Inside the MINIX kernel the $p \_n r$ field of the process descriptor is used for its IPC primitives instead of the PID.

As is was described in $\underline{\text { Chapter } 5}$, the IPC Kernel Calls use the RT-PID to identify senders and receivers RT-processes. The RT-PID is a data structure of type mrtpid_t $t$ with the following fields:

- pid: The process ID assigned by MINIX when the process was created.

- $\quad p \_n r:$ The process number assigned by the MINIX kernel when the process was created. It is related with the slot number of the kernel process table.

The IPC RT-Kernel Calls could be implemented using only the process PID to identify the RT-process. This strategy implies that the RT-kernel must scan all the process table until it finds the process that matches the specified PID. Once it find the process, the RT-kernel could get its $p \_n r$ field of the process descriptor needed for carry out the Kernel Call. This approach impose an unacceptable performance penalty for a RTOS.

The IPC Kernel Calls could be implemented using only the process $p \_n r$ to identify a RT-process, but if the RT-process terminates and other process gets the same slot number in the kernel process table, all related processes that reference to the ended process $\left(p \_n r\right)$ will refer to the new process with the same $p \_n r$. Therefore, both related field are needed to identify univocally a RT-process and to avoid an unacceptable delay.

\subsection{Adding New RT-System Calls using MRTTASK}

As MINIX4RT was designed for an academic environment, it is useful to know how to enhance its functionalities to make laboratory and coding practice. The following sections guide instructors into the steps of adding new System Calls.

To add a new RT-System Call it is appropriate to follow some system naming conventions. For a new System Call that will be named mrt_newcall:

- mrt_newcall(): RT-system call to be used by User-level programs.

- rtt_newcall(): The name of the function used by de $M M$ Server to invoke MRTTASK. 
- MRT_newcall(): The name of the function executed by MRTTASK that makes the work.

- MRT_NEWCALL: RT-System Call number.

To link each RT-System Call number with its service routine, the $M M$ server uses a System Call dispatch table (defined in $/ \mathrm{src} / \mathrm{mm} /$ table.c), which is stored in the call_vec[] array and has $N C A L L S$ entries. The $n$th entry contains the service routine address of the System Call number $n$.

To associate each function number with its corresponding service routine, the MRTTASK uses a dispatch table which is stored in the MRT_vector[] array. The $n$th entry contains the service routine address of the System Call having number $\left(M R T \_F I R S T C A L L+n\right)$.

The Table 6.1 shows some steps to add a new RT-System Call (i.e. newcall) using MRTTASK.

Table 6.1: Steps to Add a New RT-System Call.

\begin{tabular}{|c|c|c|c|}
\hline Directory & Action & File & Description \\
\hline \multirow[t]{2}{*}{ /usr/src/lib/syscall/ } & create & mrtnewcall.s & $\begin{array}{l}\text { It is an Assembler source code file. Create a label named } \\
\text { mrt_newcall to jump to a } \mathrm{C} \text { function named } \\
\text { _mrt_newcall. }\end{array}$ \\
\hline & modify & Makefile & Add the mrtnewcall.s compilation \\
\hline \multirow[t]{2}{*}{ /usr/src/lib/posix/ } & create & _mrtnewcall.c & $\begin{array}{l}\text { Create a function named_mrt_newcallo that sends a } \\
\text { message to the } M M \text { server using _syscall(MM, } \\
\text { MRTCALL, \&m) } \\
\text { The message } m \text { must have the MRTTASK operation code } \\
\left(M R T \_N E W C A L L\right) \text { in the field } m . m 1 \_i 1, m . m 2 \_i 1 \text { or } \\
m . m 3 \text { il. The other message fields can be used for the } \\
\text { MRTTASK function parameters. }\end{array}$ \\
\hline & modify & Makefile & Add the mrtnewcall.c compilation. \\
\hline \multirow[t]{2}{*}{ /usr/src/lib/syslib/ } & create & rtt_newcall.c & $\begin{array}{l}\text { Create a function named } r t \text { t_newcall() that sends a } \\
\text { message to the MRTTASK using_taskcall(MRTTASK, } \\
\text { MRT_NEWCALL,\&m). The message } m \text { have the } \\
\text { MRTTASK function parameters. }\end{array}$ \\
\hline & modify & Makefile & Add the rtt_newcall.c compilation. \\
\hline \multirow[t]{2}{*}{ /usr /include/minix/ } & modify & syslib.h & $\begin{array}{l}\text { Change MRT_NCALLS and define MRT_NEWCALL } \\
\text { Declare the function rtt_newcall() that the } M M \text { server } \\
\text { invokes to request a service from MRTTASK. }\end{array}$ \\
\hline & & com.h & Add some \#define of message fields used in mrttask.c \\
\hline \multirow{2}{*}{ /usr /include/ } & modify & unistd.h & $\begin{array}{l}\text { Add the function } m r t \text { newcallo prototype and define } \\
\text { data structures if needed. }\end{array}$ \\
\hline & modify & errno.h & Add new error types i.e. $E \_M R T \_N E W E R R O R$ \\
\hline
\end{tabular}


Table 6.1: Steps to Add a New RT-System Call (cont).

\begin{tabular}{|c|c|c|c|}
\hline Directory & Action & File & Description \\
\hline \multirow{2}{*}{$/ \mathrm{usr} / \mathrm{src} / \mathrm{mm}$} & modify & getset.c & $\begin{array}{l}\text { Change the function do_getset() To add the case } \\
M R T \_N E W C A L L \text { and the invocation to MRTTASK }\end{array}$ \\
\hline & modify & param.h & Add message fields aliases \\
\hline \multirow{2}{*}{ /usr/src/kernel/ } & modify & mrttask.c & $\begin{array}{l}\text { Add the new functions of } M R T T A S K \text {. The array of } \\
\text { function pointer } M R T \text { vector[] must be changed to point } \\
\text { to the new function } M R T \_ \text {newcall(). }\end{array}$ \\
\hline & $\begin{array}{l}\text { \# make clean; } \\
\text { make install }\end{array}$ & & Library compilation. \\
\hline /usr/src/tools & \# make hdboot & & Kernel compilation. \\
\hline
\end{tabular}

\subsection{Adding RT-Kernel Calls without Message Transfers}

Only RT-processes can make RT-System Calls direct to the RT-kernel (RT-kernel Calls) with the exception of $m r t \_s e t 2 r t()$.

The Table 6.2 shows some steps to add a new RT-Kernel Call (i.e. newkrn).

Table 6.2: Steps to Add a New RT-Kernel Call.

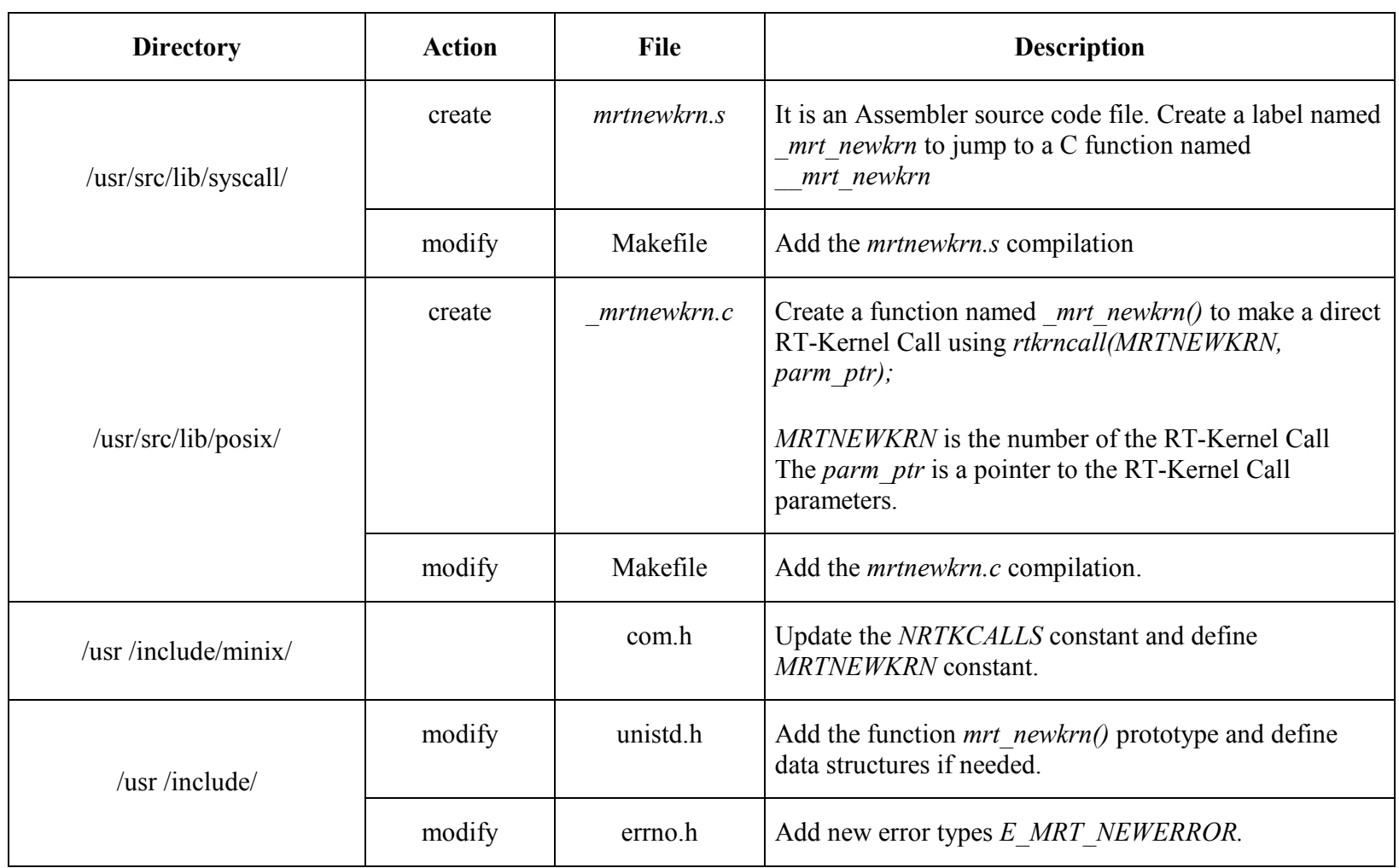


Table 6.2: Steps to Add a New RT-Kernel Call (cont).

\begin{tabular}{|c|c|c|l|}
\hline \multirow{2}{*}{ Directory } & Action & File & \multicolumn{1}{|c|}{ Description } \\
\hline \multirow{2}{*}{$/$ usr /src/kernel/ } & modify & mrtproc.c & Add the new function MRT_newkrn(). \\
\cline { 2 - 4 } & modify & mrtipc.c & Add the new function MRT_newkrn(). \\
\cline { 2 - 4 } & modify & proto.h & Add the new function MRT_newkrn() declaration. \\
\hline \multirow{2}{*}{$/$ usr/src/lib } & $\begin{array}{c}\text { \# make clean; } \\
\text { make install }\end{array}$ & & Library compilation. \\
\hline /usr/src/tools & \# make hdboot & & Kernel compilation. \\
\hline
\end{tabular}

As it was explained in the previous section, it is appropriate to follow some system naming conventions to add a new RT-Kernel Call that will be named mrt_newkrn:

- mrt_newkrn(): The RT-Kernel Call to be used by user space programs

- MRT_newkrn(): The name of the function executed by RT-kernel that do the work.

- MRTNEWKRN: RT-kernel call number

\subsection{RT-Kernel Functions}

RT-kernel functions can only be used from programs linked with the RT-kernel code. They can be used in RT-Device Drivers, RT-ISRs and RT-tasks. Table 6.3 shows a summary of RT-kernel functions.

Table 6.3: RT-Kernel Functions.

\begin{tabular}{|l|l|}
\hline \multicolumn{2}{|c|}{ Synchronization Primitives } \\
\hline void MRT_lock(void) & Disables maskable interrupts. \\
\hline void MRT_unlock(void) & Enables maskable interrupts. \\
\hline void MRT_saveFunlock(long *flags) & $\begin{array}{l}\text { Saves CPU Flags Register into a local static variable flags and } \\
\text { enable interrupts. }\end{array}$ \\
\hline void MRT_saveFlock(long *flags) & $\begin{array}{l}\text { Saves CPU Flags Register into a local static variable flags and } \\
\text { disables interrupts. }\end{array}$ \\
\hline void MRT_restoreF(long *flags) & Restores CPU Flags Register from a local static variable flags. \\
\hline
\end{tabular}


Table 6.3: RT-Kernel Functions (cont.).

\begin{tabular}{|c|c|}
\hline \multicolumn{2}{|c|}{ Synchronization Primitives } \\
\hline void MRT_enable_irq(unsigned irq) & Enables an IRQ line at the PIC 8259 controller. \\
\hline void MRT_disable_irq(unsigned irq) & Disables an IRQ line at the PIC 8259 controller. \\
\hline unsigned MRT_get_PIC(void) & Gets the PIC 8259 mask. \\
\hline void MRT_disable_irq(unsigned mask) & Sets the PIC 8259 to a specified mask. \\
\hline \multicolumn{2}{|c|}{ Interrupt Management } \\
\hline void MRT_irqd_trigger $(M R T$ irqd_t $*$ irqd $)$ & $\begin{array}{l}\text { Sets the interrupt descriptor for running in the next returning from } \\
\text { an IRQ or System Call. }\end{array}$ \\
\hline$M R T \_i r q d \_t * M R T \_i r q d \_s e r v i c e d\left(M R T \_i r q d \_t * i r q d\right)$ & $\begin{array}{l}\text { Unsets the interrupt descriptor for running in the next returning } \\
\text { from an IRQ or System Call. It must not be used after running the } \\
\text { interrupt handler because the RT-kernel do that. }\end{array}$ \\
\hline void $M R T \_i r q Q \_i n s\left(M R T \_i r q d \_t * i r q d\right)$ & Inserts an interrupt descriptor into an interrupt queue. \\
\hline void $M R T$ irqQ_rmv $\left(M R T \_i r q d \_t * i r q d\right)$ & Removes an interrupt descriptor from an interrupt queue. \\
\hline int MRT_softirq_alloc(void) & $\begin{array}{l}\text { Allocates a software interrupt descriptor. Returns the descriptor } \\
\text { number (the index of the } M R T \text { si.irqtab [] array). }\end{array}$ \\
\hline void MRT_softirq_free(int irq_nbr) & Frees a software interrupt descriptor. \\
\hline void MRT_irqd_set(int irq, MRT_irqd_t $*$ irqd) & $\begin{array}{l}\text { Sets the parameters of an interrupt descriptor (hardware and } \\
\text { software interrupts). }\end{array}$ \\
\hline void MRT_irqd_free $\left(M R T \_i r q d \_t\right.$ *irqd $)$ & $\begin{array}{l}\text { Removes a interrupt descriptor from the interrupt queue and resets } \\
\text { the descriptor fields. }\end{array}$ \\
\hline void MRT_irqd_rst $(M R T$ irqd_t $*$ *irqd $)$ & Reset all fields of an interrupt descriptor. \\
\hline \multicolumn{2}{|c|}{ Process Management } \\
\hline void $M R T \_r d y Q \_i n s\left(M R T \_p r o c \_t{ }^{*} r p\right)$ & Inserts a process descriptor at the head of a RT-ready Queue. \\
\hline void MRT_rdyQ_app $\left(M R T \_p r o c \_t \quad{ }^{*} r p\right)$ & $\begin{array}{l}\text { Appends a Process Descriptor at the tail of a RT-Ready Queue. } \\
\text { There is a macro defined in proc.h named } M R T \text { ready }) \text { that is } \\
\text { assigned to } M R T \_r d y Q \_a p p() \text {. }\end{array}$ \\
\hline void MRT_rdyQ_rmv (MRT_proc_t $\left.\quad{ }^{*} r p\right)$ & $\begin{array}{l}\text { Removes a process descriptor from a RT-ready Queue. There is a } \\
\text { macro defined in proc.h named } M R T \_u n r e a d y() \text { that is assigned to } \\
M R T \_r d y Q_{-} r m v() \text {. }\end{array}$ \\
\hline void lock_pick_proc(void) & $\begin{array}{l}\text { It is the scheduler. Its searches the RT-ready Queues for the highest } \\
\text { priority RT-process. If the queues are empty, it runs the MINIX } \\
\text { scheduler algorithm. The global variable proc_ptr is set to selected } \\
\text { process descriptor. It resets the MRT_NEEDSCHED in the global } \\
\text { system variable } M R T \text { sv.flags. }\end{array}$ \\
\hline void $M R T \_p r o c \_r s t$ (int $p \_n r$ ) & $\begin{array}{l}\text { Resets all RT parameters and statistics of a process specified in the } \\
\text { p_nr argument. }\end{array}$ \\
\hline
\end{tabular}


Table 6.3: RT-Kernel Functions (cont.).

\begin{tabular}{|c|c|}
\hline \multicolumn{2}{|c|}{ Process Management } \\
\hline int $M R T \_s e t 2 r t\left(M R T \_p r o c \_t{ }^{*} r p\right)$ & $\begin{array}{l}\text { Converts a NRT-process into a RT-process. Before call this } \\
\text { function, fill all process descriptors fields. }\end{array}$ \\
\hline int $M R T \_s e t 2 n r t\left(M R T \_p r o c \_t\right.$ & Converts a RT-process into a NRT-process. \\
\hline int MRT_sleep(struct proc *rp,long int timeout) & $\begin{array}{l}\text { Sets the MRT_P_SLEEP state flag of a process and calls } \\
M R T \text { unready(). A timeout could be specified to wakeup the } \\
\text { process at its expiration. }\end{array}$ \\
\hline int MRT_wakeup(struct proc $\left.{ }^{*} r p\right)$ & $\begin{array}{l}\text { Clears the MRT_P_SLEEP state flag of a process and calls } \\
M R T \text { ready(). }\end{array}$ \\
\hline \multicolumn{2}{|c|}{ Time Management } \\
\hline void MRT_set_timer(unsigned int Harmonic) & $\begin{array}{l}\text { Initializes channel } 0 \text { of the } 8253 \mathrm{~A} \text { timer to generate Harmonic * } \mathrm{HZ} \\
\text { interrupts by second. }\end{array}$ \\
\hline unsigned int MRT_read_timer2(void) & $\begin{array}{l}\text { Reads the channel } 2 \text { counter of the PIT. Used for latency } \\
\text { calculation. }\end{array}$ \\
\hline void MRT_vtimer_free $\left(M R T \_v t i m e r \_t * v t\right)$ & Resets all fields of a VT descriptor. \\
\hline$M R T$ vtimer_t $* M R T$ vtimer_alloc $(M R T$ vtimer_t $* v t)$ & $\begin{array}{l}\text { Allocates a VT with the parameters passed by the function } \\
\text { argument. }\end{array}$ \\
\hline void MRT_vtimer_ins $\left(M R T \_v t i m e r \_t * v t, M R T \_v t Q \_t * p t q\right)$ & Inserts a VT into a VT queue. \\
\hline $\begin{array}{l}\text { void } M R T_{-} v t i m e r \_r m v\left(M R T_{-} v t i m e r_{-} t * v t, M R T_{-} v t Q_{-} t\right. \\
\left.*_{p t q}\right)\end{array}$ & Removes a VT from a VT queue. \\
\hline \multicolumn{2}{|c|}{ Message Transfer } \\
\hline int MRT_send(int dest,int prty,int mtype) & $\begin{array}{l}\text { Sends an mtype message to a process identified by its process } \\
\text { number dest. The message priority will be prty. }\end{array}$ \\
\hline
\end{tabular}




\section{REAL-TIME PROCESSING RELATED STATISTICS}

Tools for monitoring the behavior of an operating system are invaluable in performance tuning and debugging. MINIX4RT, as a RTOS for academic uses, provides several interfaces to system and process statistics which might be used for performance analysis.

This chapter describe multiple ways of gather statistics, Appendix A is a reference that includes statistics related System Call and Kernel Call, and Appendix B gives short examples of using statistics related programming interfaces. In all cases, however, the man pages are the definitive references.

\subsection{System-wide Statistics}

The mrt_getstat() System Call let gather system-wide statistics. The address of a data structure named mrt_sysstat_s must be passed as a parameter. On return, the RT-kernel fills the following fields with statistics since the last call to mrt_RTstart() or mrt_RTrestart() System Calls:

- scheds: It counts the number of RT-schedulings.

- messages: It counts the number of messages sent (received an not).

- interrupts: It counts the number of Hardware interrupts.

- ticks: It counts the number of Timer interrupts.

- highticks: It counts the number of ticks overruns.

- idlemax: It is the highest value counted by kernel idlecount variable. (explained in Section 7.4). 
- idlelast: It is the last value counted by kernel idlecount variable. (explained in Section 7.4).

\subsection{Interrupts Service Routines Statistics}

The mrt_getistat() System Call let gather interrupt statistics. The interrupt number and the address of a data structure named $m r t \_i s t a t \_s$ must be passed as parameters. On return, the RT-kernel fills the following fields with statistics since the last call to mrt_RTstart() or mrt_RTrestart() for the specified interrupt descriptor number:

- count: It counts the the number of interrupts.

- maxshower: It is the highest number of TD-interrupts in a period.

- $\quad m d l$ : It counts the the number of Missed Deadlines.

- timestamp: It is the timestamp of the last interrupt.

- maxlat: It is the highest interrupt latency in PIT Hz units.

- reenter: It is the highest kernel reentrancy level.

\subsection{Process Statistics}

The mrt_getpstat() System Call let gather process statistics. The process RT-PID and the address of a data structure named mrt_pstat_s must be passed as parameters. On return, the RT-kernel fills the following fields with statistics for the specified process since it was converted into a RTprocess:

- scheds: The number times that the RT-process was scheduled.

- $\quad m d l:$ The number of Missed Deadlines.

- timestamp: The last schedule timestamp in PIT ticks.

- maxlat: The maximun latency to dispatch the process (not implemented yet).

- minlax: The minimun laxity for the process (not implemented yet). 
- msgsent: The number of RT-messages sent by the process.

- msgrcvd: The number of RT-messages received by the process.

Those fields match with the rtstats_t data structure of the proc data structure.

\subsection{The IDLE Process}

MINIX4RT CPU load estimation is based on the IDLE process. The IDLE process is executed when there are not any process ready to run. As more time is used by IDLE in a specified period, lower is the CPU used by the system and the other processes. IDLE is a funcion called idle_task() that jumps to MRT_idle().

$I D L E$ process uses five global variables to do its works. They are initialized by $M R T$ idle_init() Kernel Function and by the mrt_RTstart() System Call. The variables used by IDLE are:

- MRT_sv.idlecount: When the IDLE process is running, increments the value of this variable in an infinite loop. (function $M R T$ idle())

- MRT_sv.counter.idlelast: The last value of MRT_sv.idlecount before the RT-kernel resets it.

- MRT_sv.counter.idlemax: The highest value of MRT_sv.idlelast since the last execution of mrt_RTstart() or mrt_RTrestart() System Calls.

- MRT_sv.idlerefresh: A counter that is decreased on each PIT tick to control the reset action of the MRT_sv.idlecount variable. Once it is reaches zero, the RT-kernel copies MRT_sv.idlecount to MRT_sv.counter.idlelast, compares its value against MRT_sv.counter.idlemax and replaces it with the highest. Then, the RT-kernel resets MRT_sv.idlecount and copies $M R T$ sv.refresh to MRT_sv.idlerefresh.

- MRT_sv.refresh: The period to refresh IDLE counters in PIT ticks. It is specified as an argument of the mrt_RTstart() System Call. 


\subsection{The Fx Keys}

MINIX4RT as MINIX, use de Fx Keys to show statistics, attributes and status information about the system behavior. Several variables of interrupts, processes, MQs, and VTs are accessible to the user to provide data for benchmarking and testing new developments. When the user press an $F x$ $k e y$, the information is shown in the system console.

The $F 1, F 2, F 3$ and $F 5$ keys are used by MINIX. F1 shows MINIX processes attributes, $F 2$ shows MINIX processes memory map, F3 Toggle scrolling mode and $F 5$ shows Ethernet statistics.

\subsubsection{The Shift-F1 Hot-Key}

The Shift-F1 Hot-Key displays the RT-attributes of all running processes. In the last line of Figure 7.1 the process named mrttest $8 c$ that has the PID 27 is a RT-process (FLAGS $=4002$ ) waiting to receive a message from process $6(\mathrm{RCVF}=6)$. Its efective priority is $4(\mathrm{PRTY}=4)$ and its base priority is 4 too $(\mathrm{BASE}=4)$.

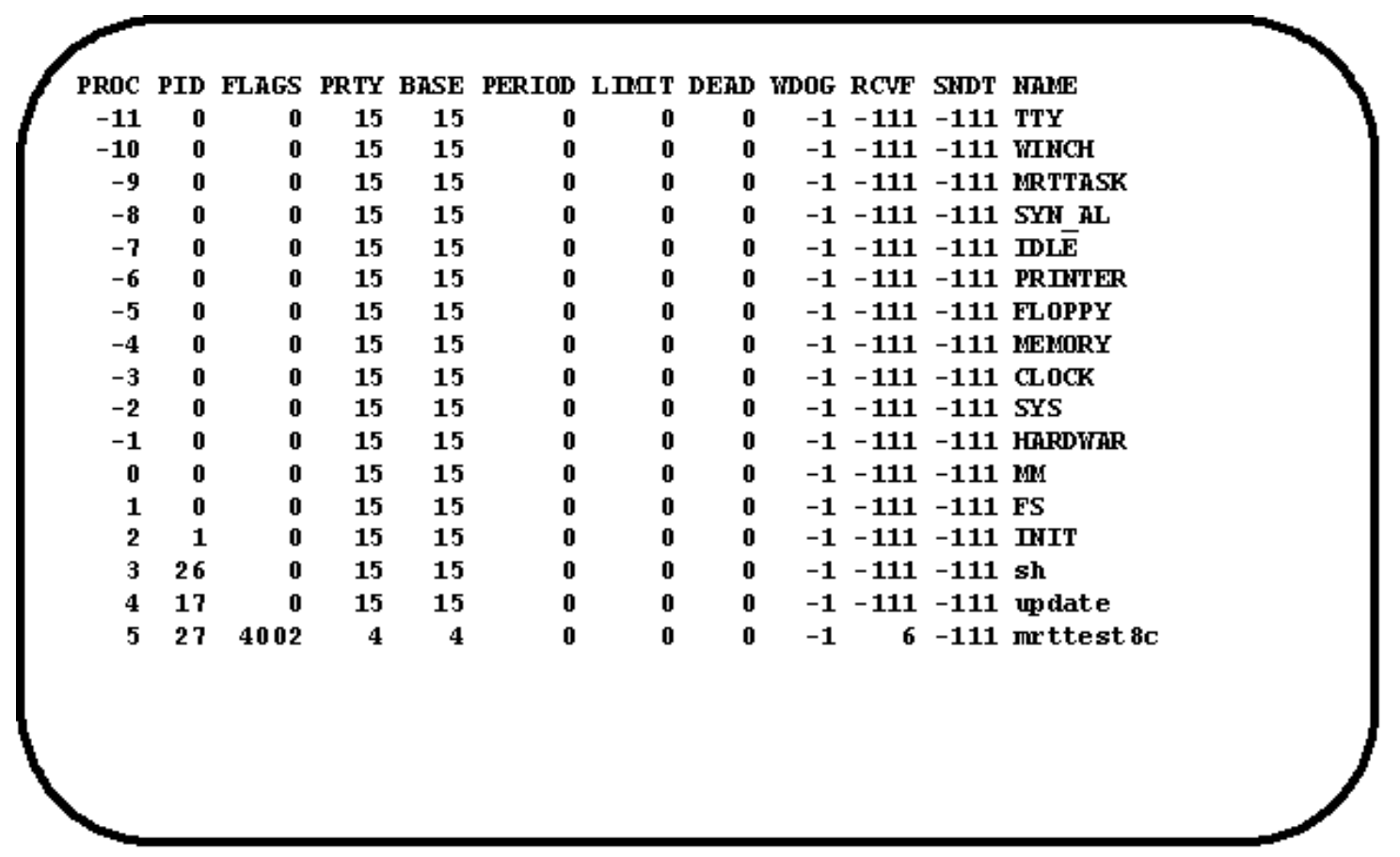

Figure 7.1: RT-Process Attributes.

The columns displayed have the following meanings:

- PROC: The process number. 
- PID: The Process Identifier.

- FLAGS: The process' RT-status flags.

- PRTY: The process' effective priority.

- BASE: The process' base priority.

- PERIOD: The process' period specified in PIT ticks (only for MRT_P_PERIODIC).

- LIMIT: The limit for the number of RT-schedulings.

- DEAD: The process deadline specified in PIT ticks.

- WDOG: The process' watchdog process.

- RCVF: The process from which the process wants to receive a RTmessage.

- SNDT: The process to which the process wants to send a RT-message.

- NAME: The name of the process.

\subsubsection{The Ctrl-F1 Key}

The Ctrl-F1 Hot-Key displays the RT-statistics of all running processes. In the last line of Figure 7.2 a process named mrttest $8 c$ was scheduled 10 times (SCHEDS=10), the last was at time 32753 ticks (TSTAMP $=32753$ ) since the last $m r t$ RTstart()/mrt_restart() system call invocation. The columns displayed have the following meanings:

- PROC: The process number. The first process is (-NR_TASKS).

- PID: The Process Identifier.

- SCHED: Counts how many times the process has been selected by the RT-scheduler.

- $M D L:$ The number of Missed Deadlines.

- TSTAMP: The last schedule timestamp in PIT ticks. 
- MAXLAT: The maximun latency to dispatch the process (not implemented yet).

- MINLAX: The minimun laxity for the process (not implemented yet).

- SENT: The number of RT-messages sent by the process.

- $\quad$ RCVD: The number of RT-messages received by the process.

- VT: The VT assigned to a Periodic process.

- $\quad M Q$ : The MQ assigned to a RT-processes.

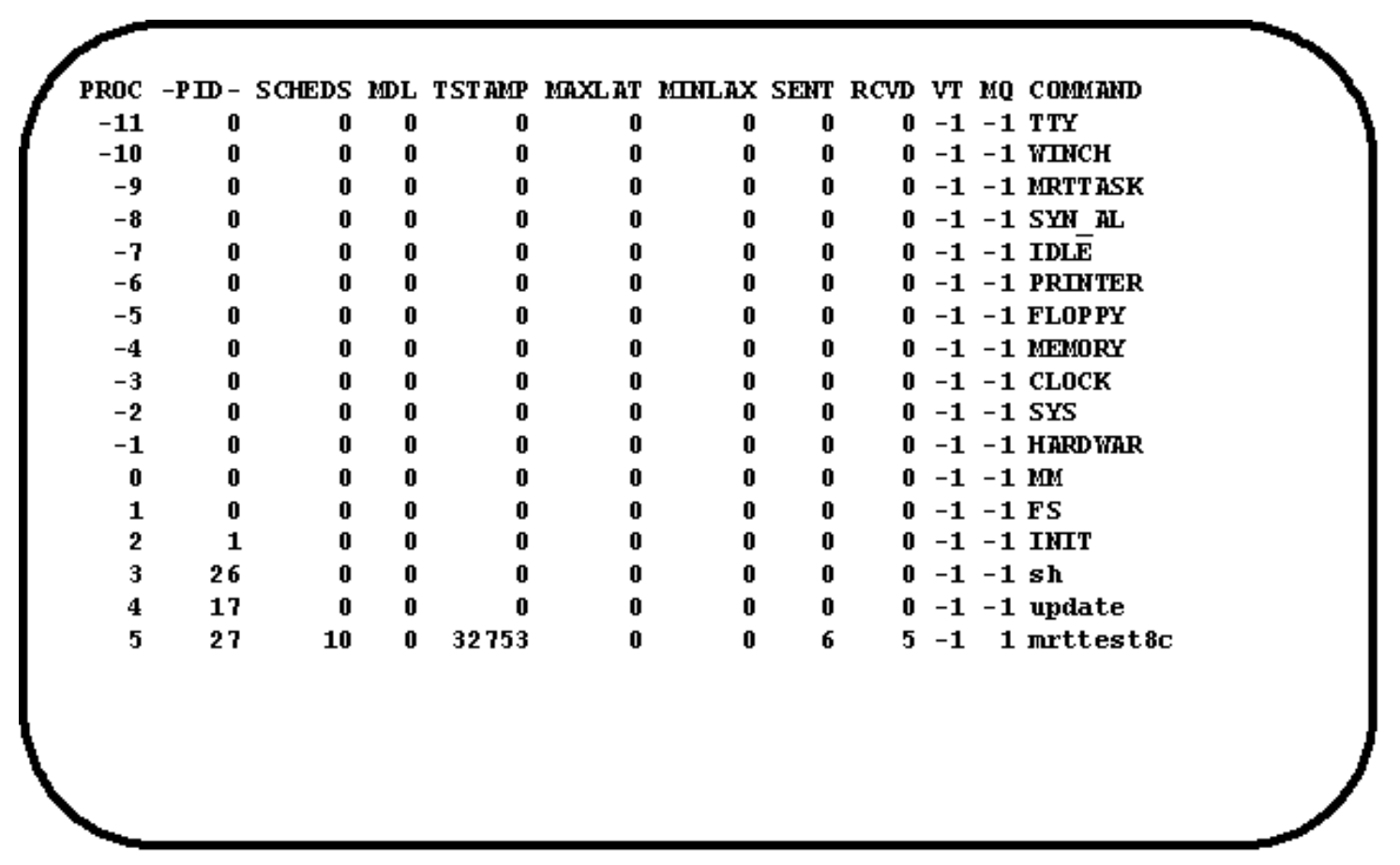

Figure7.2: RT-Processes Statistics.

\subsubsection{The Shift-F2 Hot-Key}

PIT Latency between 2 reads: is the time in PIT Hz, between two sequencial reads of the PIT LATCH counter. It is useful to estimate the time consumed by a read operation of the PIT LATCH used as was explained in Chapter 4.

The other system status, counters and statistics are simple to understand that not need further explanation (see Figure7.3). 
Total number of interrupts $=117579$

Total number of RT scheds $=23$

Total number of RT messages $=10$

Free messages desc. in Pool $=32 / 32$

Total number of RT ticks $=109588$

RT ticks/s $\quad=200$

MTIX ticks/s $=50$

PIT Latency between 2 reads $=14$

Processing Mode Flags $=0000000000000001$

Bitmap of hard ints in use $=0100000011011011$

Bitmap of soft ints in use $=000000000000001$

Bitmap of MrIX virtual PIC $=1011111100100000$

Bitmap of the REAL PIC $=1011111100100000$

MRT_sv.counter.idlelast $\quad=8059642$

MRT sv. counter.idlemax $=8087867$

cPU Load

Sizeof mrt_msg_t $\quad=28$

Figure 7.3: RT-System Wide Attributes and Statistics.

\subsubsection{The F4 Key}

The F4 Key displays the RT-attributes, status and statistics of all RT-Hardware interrupt descriptors as is shown in Figure 7.4. The columns displayed have the following meanings:

- IRQ: The interrupt descriptor number.

- TSK: The RT-Task number related with the interrupt.

- WDG: The watchdog process number.

- COUNT: It counts the number of interrupts.

- MLAT: Maximun Latency of the interrupt handler.

- LAT: The last latency of the interrupt handler.

- $R E$ : The interrupt descriptor reenter field.

- PY: The interrupt priority.

- $\quad$ TY: The interrupt type. 
- VT: The VT assigned to the interrupt descriptor (only for TD-interrupts).

- TSTAMP: The timestamp of the last handler execution.

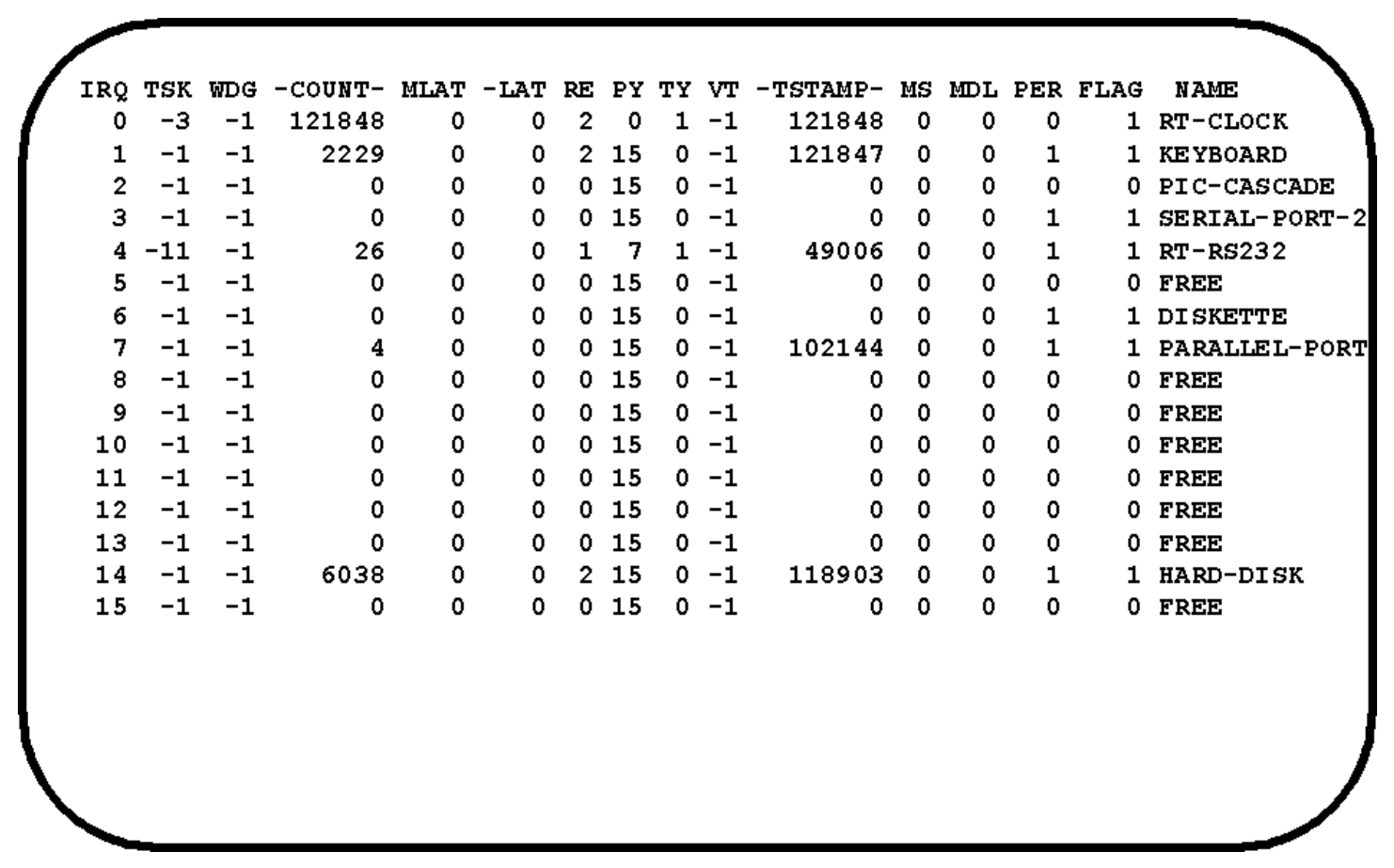

Figure 7.4: Hardware Interrupt Descriptors.

- MS: The maximun value of shower field of the interrupt descriptor.

- $\quad M D L:$ The number of missed deadlines.

- PER: The period of the interrupt (only for TD-interrupts).

- FLAG: The status flags of the interrupt descriptor.

- NAME: The name of the interrupt descriptor.

Figure 7.4 shows that IRQ 4 is a Timer Driven RT-interrupt descriptor $(\mathrm{TY}=1)$ named RT-RS232 with a priority of $7(\mathrm{PY}=7)$ and a period of $1(\mathrm{PER}=1)$ tick. The handler was executed 26 times, the last at 49006 ticks since the last mrt_RTstart()/mrt_restart() system call invocation.

\subsubsection{The Shift-F4 Hot-Key}

The Shift-F4 Hot-Key displays the RT-attributes, status and statistics of all Software interrupt descriptors. The attributes, status and statistics displayed are the same as for the F4 
key. Figure 7.5 shows the sofware IRQ 16 dedicated to manage the MINIX clock (timer) virtualization. The handler was executed 31215 times since the last mrt_RTstart()/mrt_restart() system call invocation.

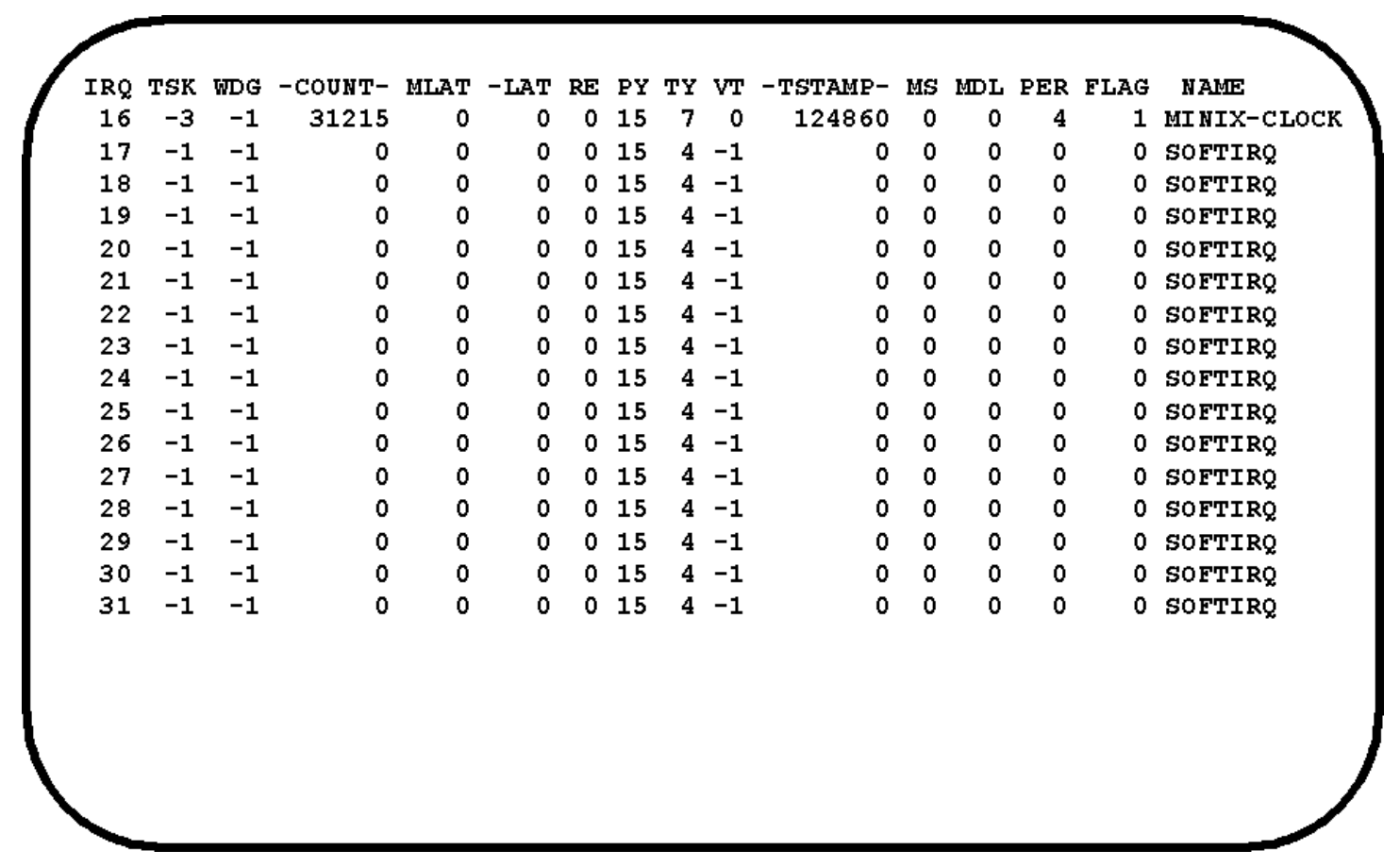

Figure 7.5: Software Interrupt Descriptors.

\subsubsection{The Ctrl-F4 Hot-Key}

The Ctrl-F4 Hot-Key displays the interrupt descriptors queues and the bitmap of Interrupt Queues as is shown in Figure 7.6. The columns displayed have the following meanings:

- PRI: The priority of the interrupt descriptor.

- INQ: The current number of descriptors enqueued.

- PEND: The number of pending interrupt descriptors.

- Enqueued Interrupt Descriptors: The list of Interrupt descriptors for each queue. 
Interrupt Pending Queues bitmap $=0000000000000000$

PRI IHQ PEKD Enqueued Interrupt Descriptors

$\left.\begin{array}{lllll}0 & 1 & 0 & \Rightarrow[ & 0\end{array}\right]$

100

200

300

400

$\begin{array}{lll}5 & 0 & 0 \\ 6 & 0 & 0\end{array}$

$\left.\begin{array}{lllll}7 & 1 & 0 & \Rightarrow\end{array}\right]$

800

900

1000

1100

1200

1300

1400

$1560 \Rightarrow\left[\begin{array}{ll}1 & 6\end{array}\right] \Rightarrow\left[\begin{array}{ll}3 & 3\end{array}\right] \Rightarrow\left[\begin{array}{ll}6 & 6\end{array}\right] \Rightarrow\left[\begin{array}{ll}7\end{array}\right] \Rightarrow\left[\begin{array}{ll}14\end{array}\right] \Rightarrow>[16]$

Figure 7.6: Interrupt Descriptors Queues.

\subsubsection{The Shift-F5 Hot-Key}

The Shift-F5 Hot-Key displays the RT-ready processes queues and MINIX ready processes queues and the RT-ready queue bitmap.

Priority Queues bitmap =

0000000000010000

PRI MAXIPO IHQ PROC-LIST

0 0 0

100

200

300

$\begin{array}{lllll}4 & 2 & 1\end{array}$

500

$6 \quad 0 \quad 0$

700

8 0 10

$\begin{array}{rrr}9 & 0 & 0 \\ 10 & 0 & 0\end{array}$

1100

1200

1300

14000

1500

PRI MIIIX-PROC-LIST

$0->[0]$

1

2

Figure 7.7: RT-Ready Queues and MINIX Ready Queues. 
Figure 7.7 shows process number 7 as the only process in the RT-ready queue of priority 4 . This queue has got 2 process enqueued.

The columns displayed have the following meanings:

- PRI: The priority of RT-ready processes queue.

- MAXINQ: The maximun number of descriptors enqueued.

- INQ: The current number of descriptors enqueued.

- PROC-LIST: The current list of RT-ready processes in each queue.

- MINIX-PROC-LIST: The current list of MINIX ready processes in each queue.

\subsubsection{The F6 Key}

The F6 Key displays the RT-message pool with the messages attributtes. The columns displayed have the following meanings:

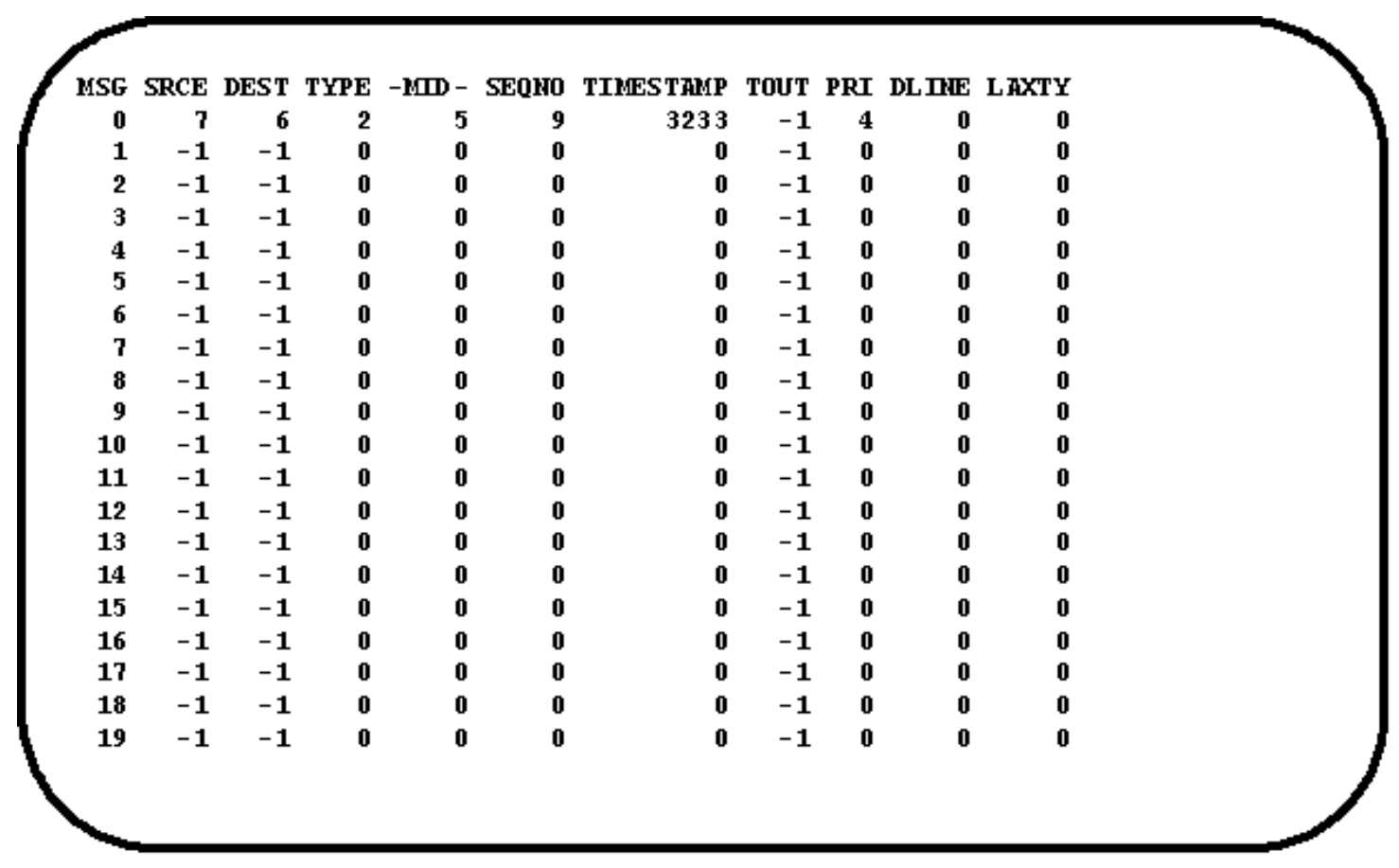

Figure 7.8: RT-message Pool (Message Attributtes).

- MSG: The message queue entry number in the array MRT_sm.mpool.mqe[]. 
- SRCE: The message source process.

- DEST: The message destination process.

- TYPE: The message type described in include/minix/const.h.

- MID: The system wide message ID.

- SEQNO: The source process message sequence number.

- TIMESTAMP: The message sent timestamp.

- TOUT: The message timeout.

- PRI: The message sender priority.

- DLINE: The message sender deadline.

- LAXTY: The message sender laxity.

Figure 7.8 shows message number 0 with a timestamp of 3233 from source 7 and destination 6 with a message ID of 2, a sequence number of 9 and a priority of 4 .

\subsubsection{The Shift-F6 Hot-Key}

The Shift-F6 Hot-Key displays RT-message pool with messages contents. The first line of Figure 7.9 shows message number 1 from source 7 and destination 6 with a contents of "HELLO FATHER".

The columns displayed have the following meanings:

- MSG: The message queue entry number in the MRT_sm.mpool.mqe[] array.

- SRCE: The message source process.

- DEST: The message destination process.

- TYPE: The message type described in include/minix/const.h.

- MID: The system wide message ID. 
- VT: The VT assigned to the message.

- 01234567890123456789: The message content.

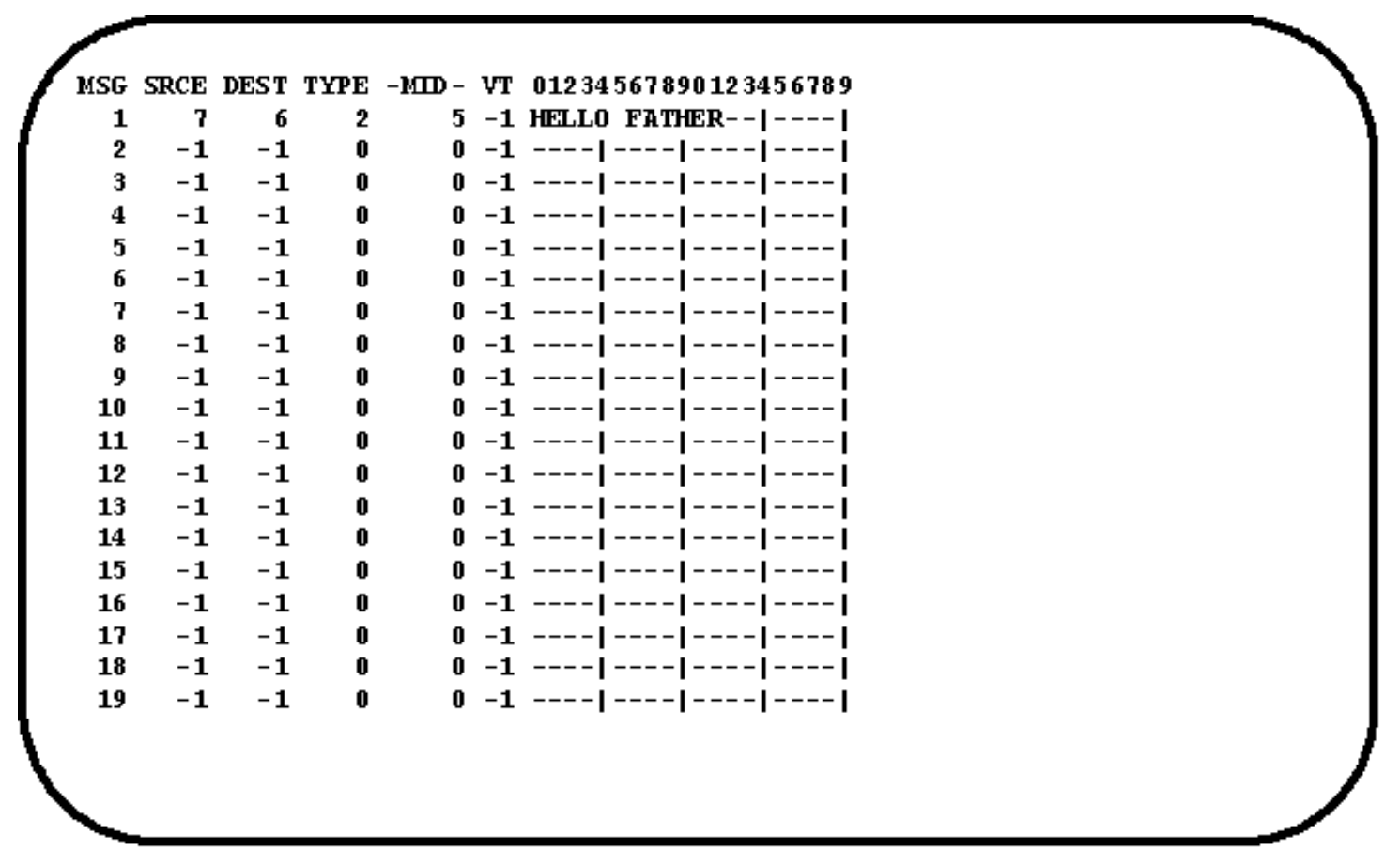

Figure 7.9: RT-Message Pool (Message Contents).

\subsubsection{The Ctrl-F6 Hot-Key}

The Ctrl-F6 Hot-Key displays MQ Status an Statistics. The columns displayed have the following meanings:

- ID: The MQ number in the $M R T \_s m . m s g Q[]$ array.

- $\quad$ SZ: The MQ size (in MQEs).

- FLAG: The MQ status and policy flags.

- BITM: The MQ priority bitmap.

- INQ: The number of message enqueued.

- MAX: The maximun number of message enqueued.

- OWN: The MQ owner. 
- VT: The assigned VT for the MQ.

- DLVD: The total number of messages delivered.

- ENQD: The total number of messages enqueued.

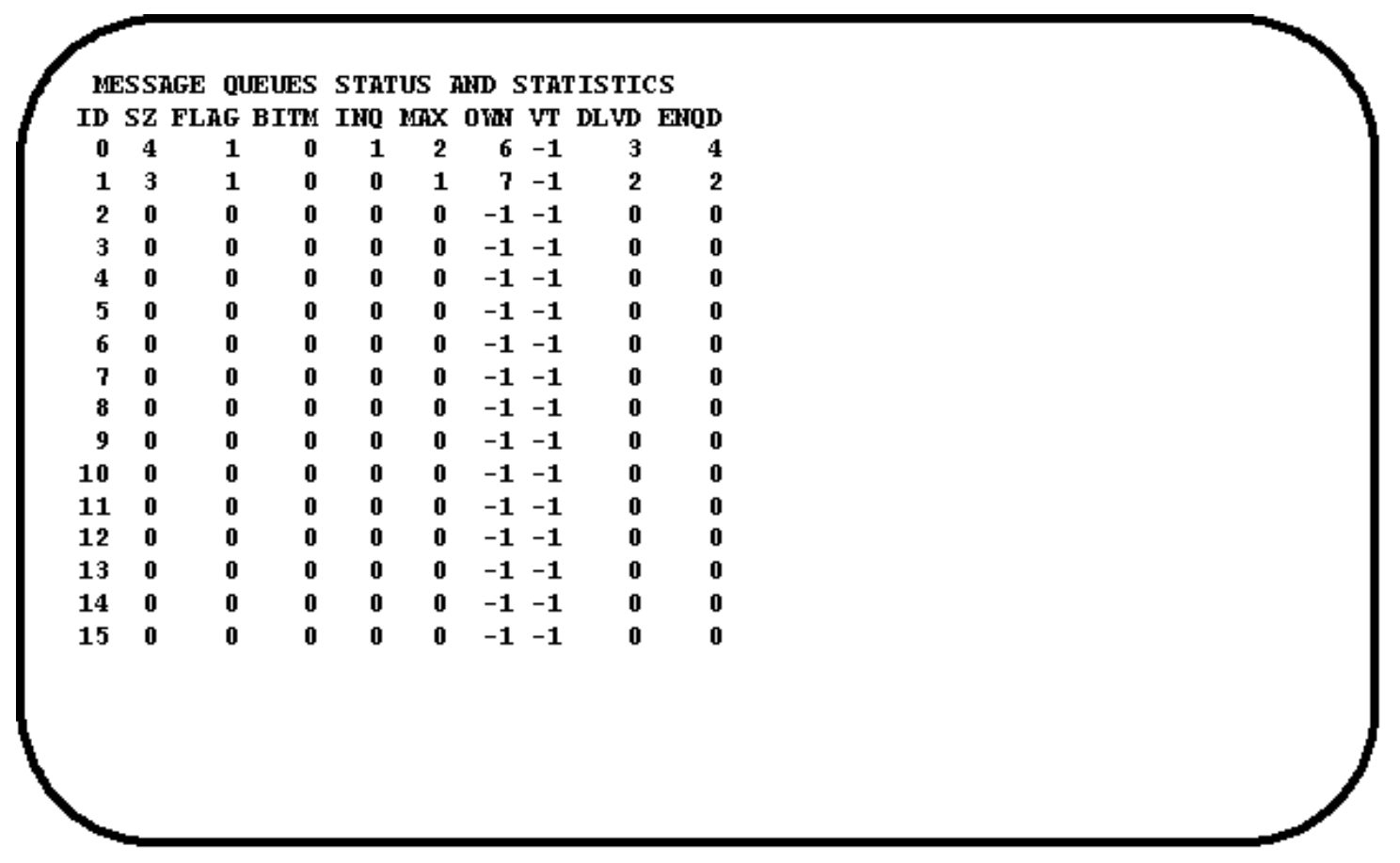

Figure 7.10: Message Queue Status and Statistics.

Figure 7.10 shows that Message Queue with ID 0 have a size of 4 messages with one message enqueued. Its owner is process number 6 and it has enqueued 4 messages and delivered 3 messages.

\subsubsection{The F8 Key}

The F8 Key displays VT attributes, status and statistics.Figure 7.11 shows VT number 0 that is the emulated MINIX CLOCK interrupt with a period of 4 and action type 4 (MRT_ACT_IRQTRIG) with an action parameter 16 is owned by process -3 (the CLOCK Task) with priority 15. It has expired 37461 times and the last expiration was at tick 149844.

The columns displayed have the following meanings:

- VT: VT number.

- PERIOD: VT period in PIT ticks. 
- NEXTEXP: Next VT expiration.

- LIMIT: A limit for VT expirations.

- ACT: The action of the VT.

- PAR: The VT parameter field.

- NR: Same as the VT number.

- OWN: The owner process of the VT.

- PRTY: The VT priority.

- EXPIRE: The number of VT expirations.

- TIMESTAMP: The last VT expiration timestamp.

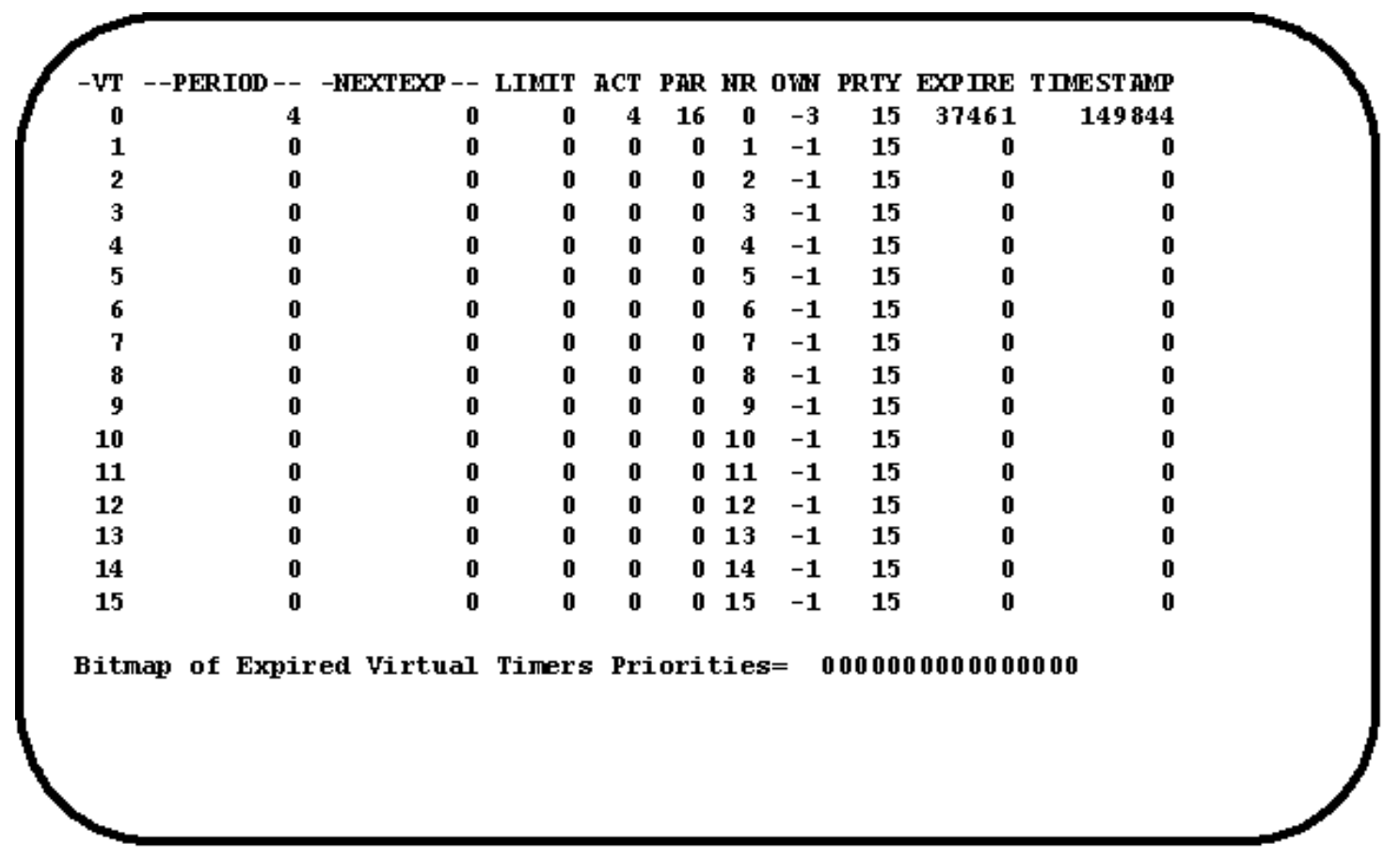

Figure 7.11: Virtual Timer Attributes, Status and Statistics.

\subsubsection{The Shift-F8 Hot-Key}

The Ctrl-F8 Hot-Key displays RT-Virtual timer queues status. Figure 7.12 shows the Active Queue that have VT number 0 enqueued and 15 Expired Queues, one queue for each system priority. The last line shows the Free VT Queue that have 15 free VTs. 
The columns displayed have the following meanings:

- TYPE: The VT queue type

- PRTY: The Priority of the VT queue

- INQ: The number of VTs enqueued

- MAXINQ: The maximun number of VTs enqueued.

- LIST: The lists of VTs.

- $A C T V$ : It is the Active VT queue.

- EXPD: It is an Expired VT queue.

- FREE: It is the Free VT queue.

TYPE PRTY TIO MAXTIO LIST

ACTV HLL 1 3 $-->$ [0] $->$ [HULL]

EXPD 0 0 0 0

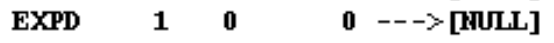

EXPD $2 \begin{array}{llll}2 & 0 & 0 & --\rightarrow \text { [RULL] }\end{array}$

$\begin{array}{llllll}\text { EXPD } & 3 & 0 & 0 & -->\text { [RULL] }\end{array}$

EXPD $4 \quad 0 \quad 0 \quad 1-\cdots$ [NULL]

EXPD 5000010

EXPD $6 \begin{array}{llll}6 & 0 & 0 & -->\text { [NULL] }\end{array}$

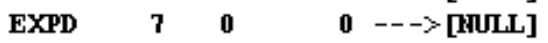

$\begin{array}{lllll}\text { EXPD } & 8 & 0 & 0 & -->\text { [HULL] }\end{array}$

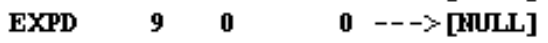

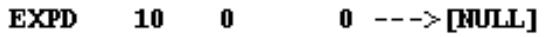

EXPD $11 \quad 0 \quad 00-->$ [MULL]

$\begin{array}{lllll}\text { EXPD } & 12 & 0 & 0 & -->\text { [NULL] }\end{array}$

EXPD $13 \quad 0 \quad 00-->$ [HULL]

EXPD 14 0 1500

EXPD $15 \quad 0 \quad 1-->$ [MULL]

FREE HOHE $1516-\rightarrow>[12] \rightarrow[13]->[14] \rightarrow[15] \rightarrow[1] \rightarrow[2] \rightarrow[3]->[4]->[5]->[6] \rightarrow[$ $7] \rightarrow[8]->[9]->[10]->[11]->[$ MULL $]$

Figure 7.12: RT-Virtual Timer Queues. 


\subsection{The Modified ps Command}

The $p s$ command reports information about active processes. It was modified to support new options to show processes statistics and attributes related to RT-processing. The columns have the same meanings of fields explained in previous sections.

\subsubsection{The -A Option}

This option shows process attributes related to RT-processing (see Figure 7.13).

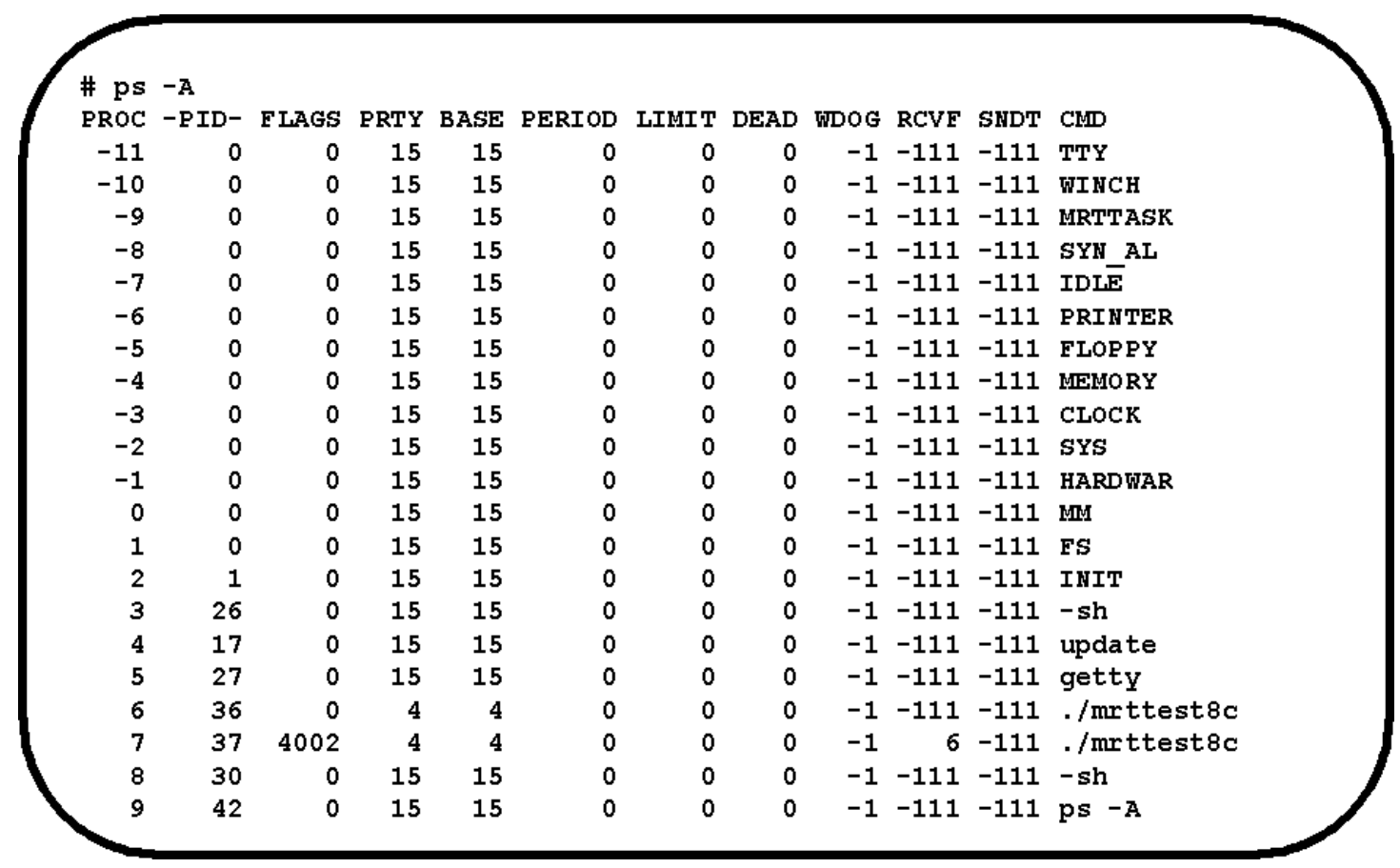

Figure 7.13: ps Command with -A Option.

\subsubsection{The $-\mathrm{S}$ Option}

This option shows process statistics related to RT-processing. Figure 7.14 shows process number 6 named mrttest $8 c$ with PID 36 has been scheduled 12 times, the last schedule at time 22314 ticks since the last mrt_RTstart()/mrt_restart() System Call invocation. 


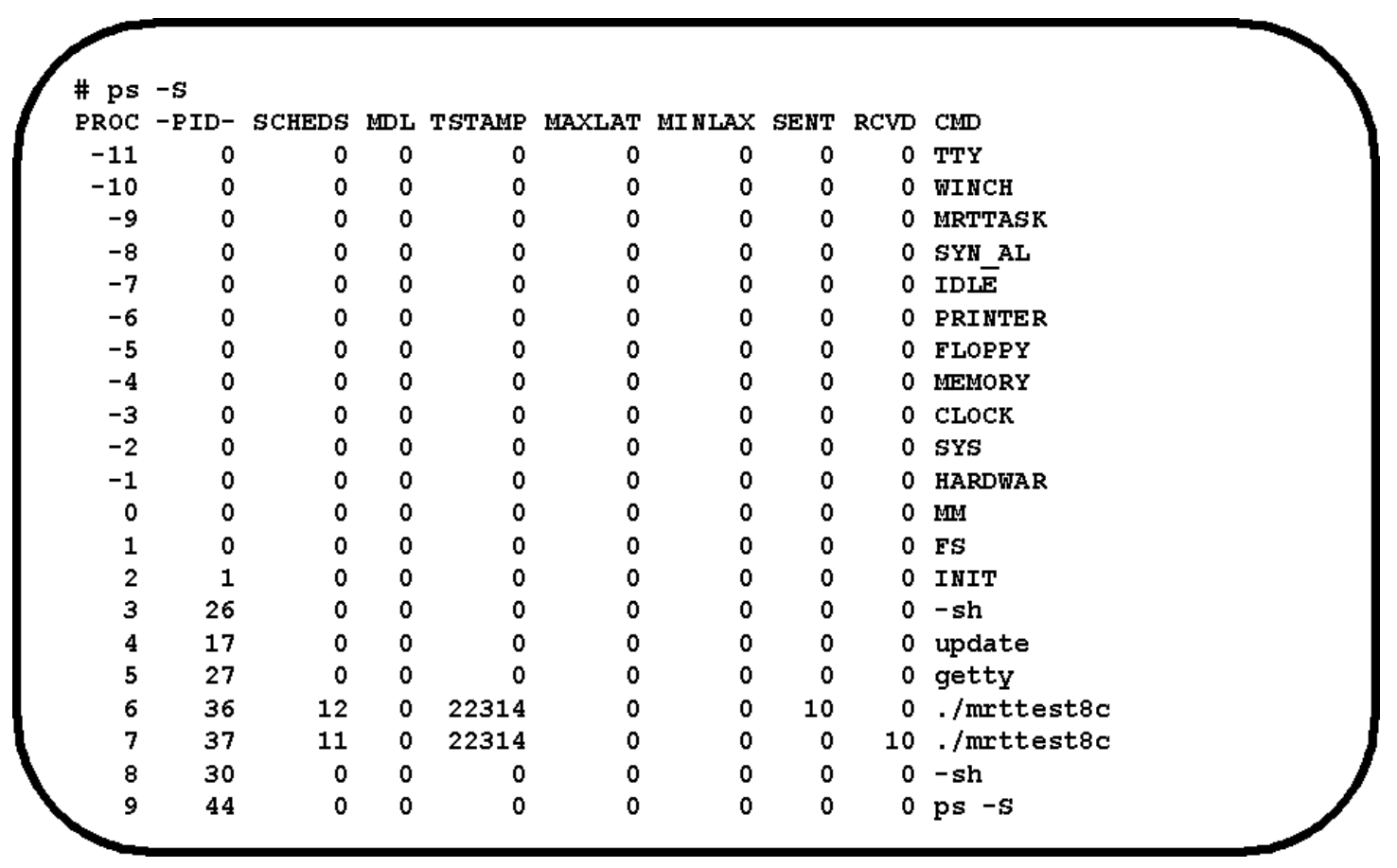

Figure 7.14: ps Command with -S Option.

\subsection{The mrtstatus Command}

The mrtstatus command provides additional information related to RT-processing. As the $p s$ command, it uses the character special files /dev/mem and /dev/kmem MINIX devices that map system and kernel memory to files. Both devices, the /dev/null, and RAM disks are supported by the MEMORY Task.

The MEMORY Task offers several IOCTL operations on memory devices. The MIOCGPSINFO is used by the MM and the FS to gather information about the addresses of their process tables needed by the $p s$ command.

MINIX4RT adds a new IOCTL operation to the MEMORY Task named MIOCGMRTINFO to gather information about the addresses of main RT-kernel tables and data structures needed by the mrtstatus command. The mrtstatus command reports:

- RT-kernel constants.

- System-wide statistics.

- Interrupt descriptor statistics.

- Virtual Timer statistics. 
- Messages statistics.

- Interrupt Queue statistics.

- Virtual Timer statistics.

- Message Queues statistics.

The several columns of the reports have the same meanings of fields explained in previous sections, other will be described in the following subsections.

\subsubsection{The -s Option}

This option (default) shows System-wide statistics related to RT-processing (see Figure 7.15).

$\begin{array}{ll}\text { \# mrtstatus } & \\ \text { flags } & =1 \\ \text { virtual_PIC } & =\text { BF20 } \\ \text { PIT_latency } & =16 \\ \text { PIT_latch } & =5965 \\ \text { tickrate } & =200 \\ \text { harmonic } & =4 \\ \text { refresh } & =200 \\ \text { scheds } & =23 \\ \text { messages } & =10 \\ \text { interrupts } & =34935 \\ \text { ticks } & =31485 \\ \text { highticks } & =0 \\ \text { idlemax } & =8087325 \\ \text { idlelast } & =8080760 \\ & \end{array}$

Figure 7.15: mrtstatus Command with -s Option. 


\subsubsection{The -i Option}

This option shows interrupt descriptors status and statistics (see Figure 7.16).

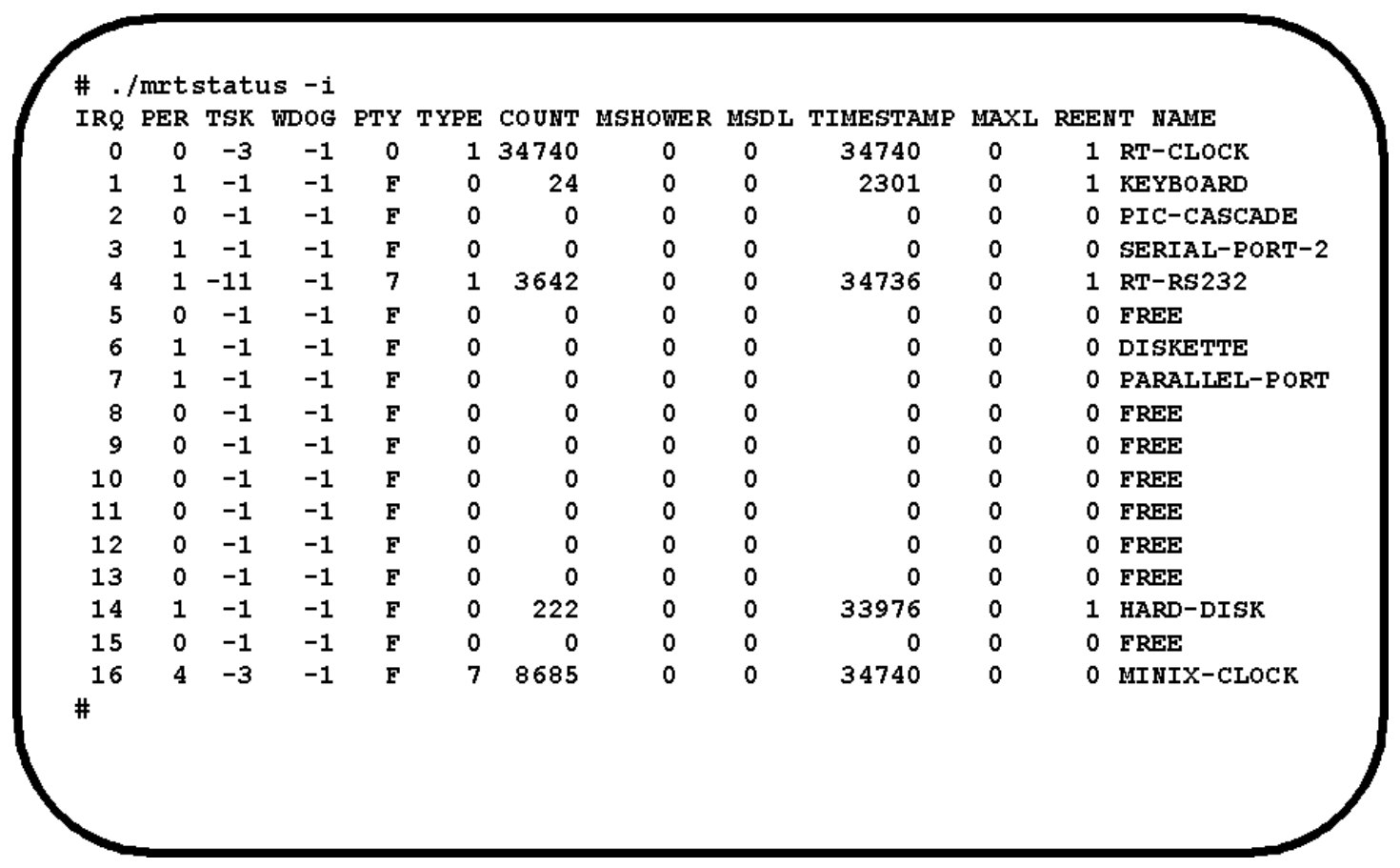

Figure 7.16: mrtstatus Command with -i Option.

\subsubsection{The -t Option}

This option shows VTs status and statistics (see Figure 7.17). The columns displayed have the following meanings:

- VT: VT number.

- PERIOD: VT period in PIT ticks.

- NEXTEXP: Next VT expiration.

- LIMIT: A limit for VT expirations.

- ACT: The action of the VT.

- PAR: The VT parameter field.

- $\quad N R$ : Same as the VT number. 
- OWN: The owner process of the VT.

- PRTY: The VT priority.

- EXPIRE: The number of VT expirations.

- TIMESTAMP: The last VT expiration timestamp.

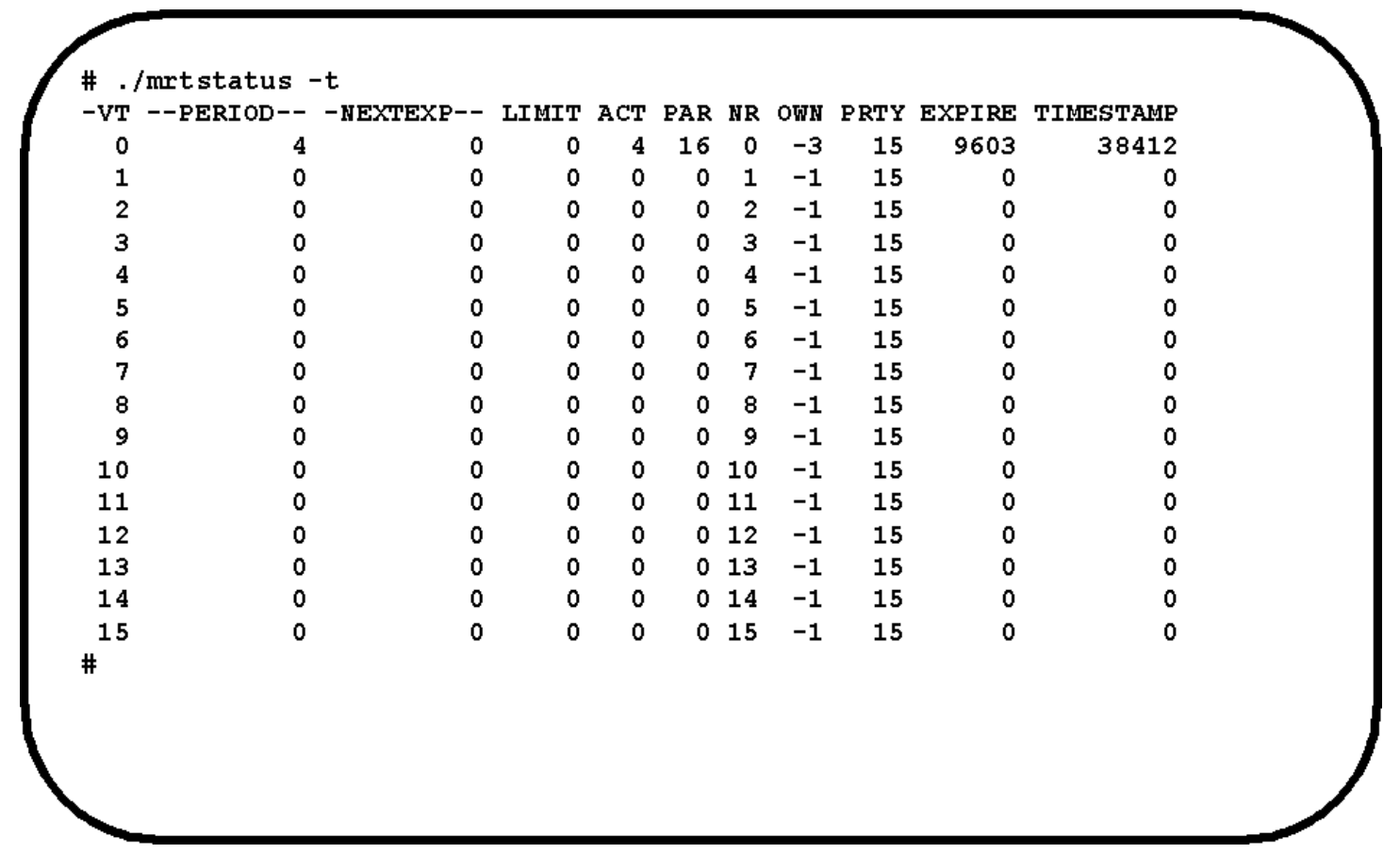

Figure 7.17: mrtstatus Command with -t Option.

\subsubsection{The - m Option}

This option shows messages status and statistics (see Figure 7.18). The columns displayed have the following meanings:

- $M S G$ : Message number.

- SPID/SNBR: Source PID and Source Number (RT-PID).

- $\quad D P I D / D N B R$ : Destination PID and Destination Number (RT-PID).

- TYPE: Message Type.

- MID: Message ID.

- SEQNO: Sequence Number. 
- TIMESTAMP: Message timestamp.

- PRI: Message Priority.

- DLINE: Message Sender Deadline.

- LAXTY: Message Sender Laxity.

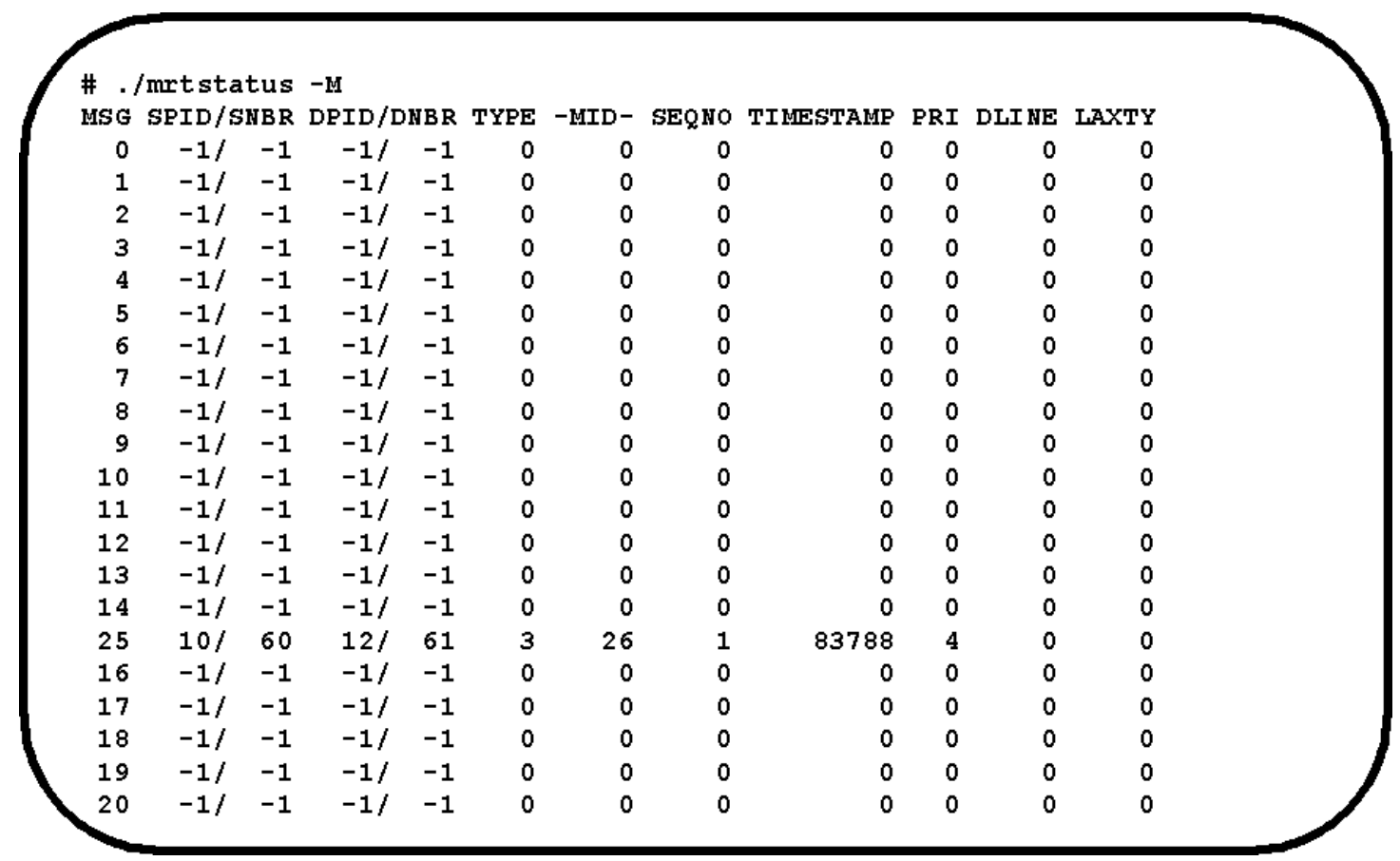

Figure 7.18: mrtstatus Command with -m Option.

\subsubsection{The -c Option}

This option shows the values of RT-kernel constant (see Figure 7.19).

The constants showed have the following meanings:

- NR_VTIMERS: Number of system Virtual Timers.

- NR_PRTY: Number of priority levels.

- NR_IRQ_VECTORS: Number of IRQ vectors (and Hardware interrupt descriptors).

- NR_IRQ_SOFT: Number of Software interrupt descriptors. 
- NR_MSGQ: Number of Message Queues (It limits the number of RTprocesses)

- NR_MESSAGES: Number of system messages.

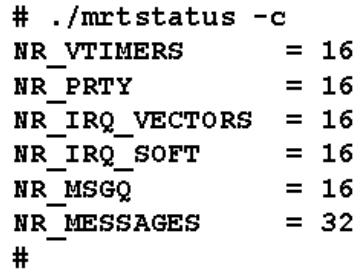

$=32$

Figure 7.19: mrtstatus Command with -c Option.

\subsubsection{The -I Option}

This option shows interrupt queues status and statistics (see Figure 7.20).

The columns displayed have the following meanings:

- PRI: The priority of the interrupt descriptor.

- INQ: The current number of descriptors enqueued.

- PEND: The number of pending interrupt descriptors.

Each bit set in the bitmap displayed represents an interrupt queue with at least one descriptor triggered (service pending). 


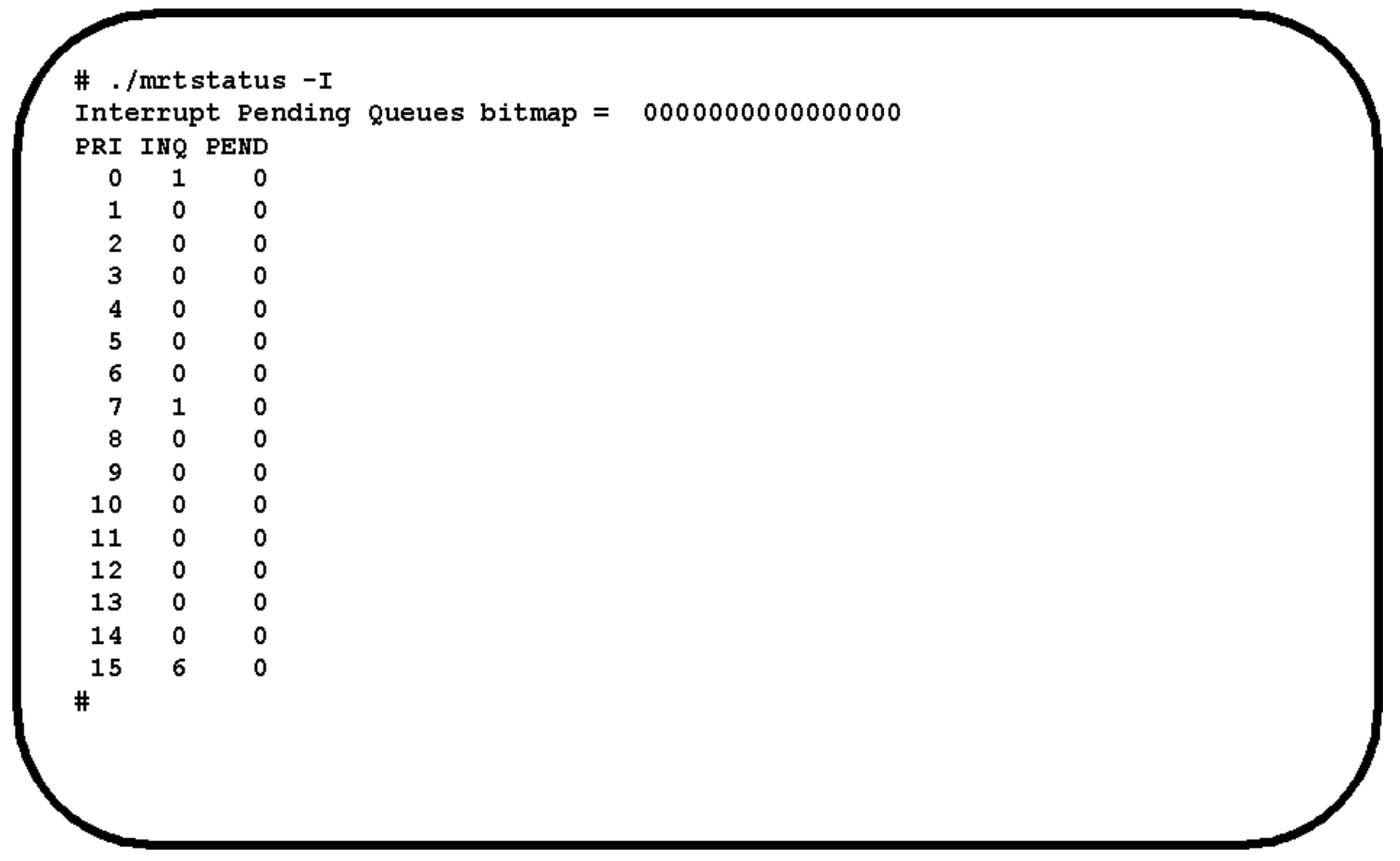

Figure 7.20: mrtstatus Command with -I Option.

\subsubsection{The -T Option}

This option shows VTs queues status and statistics (see Figure 7.21).

The columns displayed have the following meanings:

- $\quad$ TYPE: The VT queue type

- PRTY: The Priority of the VT queue

- INQ: The number of VTs enqueued

- MAXINQ: The maximun number of VTs enqueued.

- $A C T V:$ It is the Active VT queue.

- $\quad E X P D:$ It is an Expired VT queue.

- FREE: It is the Free VT queue. 


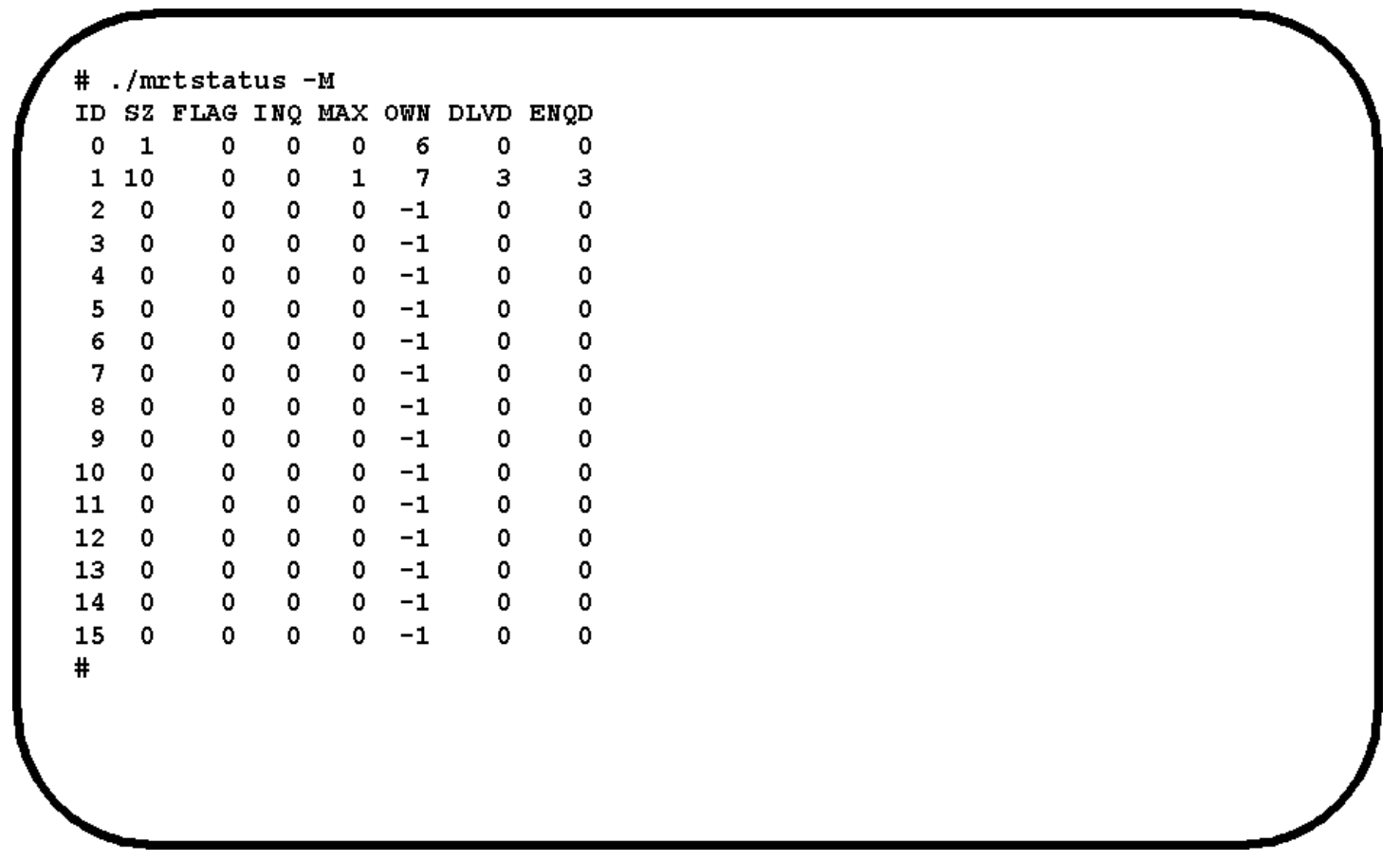

Figure 7.21: mrtstatus Command with -T Option.

\subsubsection{The -M Option}

This option shows MQ status and statistics (see Figure 7.22).

\begin{tabular}{lrrr} 
\# . $r$ mrtitus & \multicolumn{1}{c}{ T } \\
TYPE & PRTY & INQ & MAXINQ \\
ACTV & ALL & 2 & 3 \\
EXPD & 0 & 0 & 0 \\
EXPD & 1 & 0 & 0 \\
EXPD & 2 & 0 & 0 \\
EXPD & 3 & 0 & 0 \\
EXPD & 4 & 0 & 1 \\
EXPD & 5 & 0 & 0 \\
EXPD & 6 & 0 & 0 \\
EXPD & 7 & 0 & 0 \\
EXPD & 8 & 0 & 0 \\
EXPD & 9 & 0 & 0 \\
EXPD & 10 & 0 & 0 \\
EXPD & 11 & 0 & 0 \\
EXPD & 12 & 0 & 0 \\
EXPD & 13 & 0 & 0 \\
EXPD & 14 & 0 & 0 \\
EXPD & 15 & 0 & 1 \\
FREE & NONE & 14 & 16 \\
\# & & &
\end{tabular}

Figure 7.22: mrtstatus Command with -M Option. 
The columns displayed have the following meanings:

- ID: The MQ number in the MRT_sm.msgQ[] array.

- $\quad$ SZ: The MQ size (in MQEs).

- FLAG: The MQ status and policy flags.

- INQ: The number of message enqueued.

- MAX: The maximun number of message enqueued.

- OWN: The MQ owner.

- $\quad D L V D$ : The total number of messages delivered.

- ENQD: The total number of messages enqueued. 


\section{CONCLUSIONS AND FUTURE WORKS}

\subsection{Conclusions}

MINIX has proved to be a feasible testbed for OS development and extensions that could be easily added to it. In a similar way, MINIX4RT has an architecture that can be used as a starting point for adding RT-services.

In spite of it was designed for an academic environment, it can be optimized for production systems even in embedded systems. Its Virtual Machine architecture, code readability, MINIX compatibility and the similarities of several of its algorithms and data structures helps to minimize the understanding time of its source code. Those characteristics make it suitable for course assignments and RT-project developments as the support of Rate Monotonic/Deadline Monotonic scheduling algorithms, Sporadic/Deferable Servers implementation and performance evaluation tests.

MINIX4RT microkernel has basic features as Interrupt Management, Process Management, Time Management, Real-Time IPC and Statistics gathering making it a good choice to conduct coding experiences. Device-drivers writers have at their disposal several flavors of interrupt handling as Event-Driven, Timer-Driven, Software and Non-Real-Time Interrupt Service Routines execution.

\subsection{Future Works}

MINIX4RT development does not finish with this thesis. There are other planed projects for its improvement as:

- RT-FIFOs: They are mechanisms equivalent to RTLinux RT-FIFOs that permit RT-processes communicate with NRT-processes. 
- RT-Semaphores: They are known mechanisms in OSs used for synchronization and mutual exclusion among RT-processes.

- Non Periodic Time Management: Often, OSs increase the Timer frequency when they need better time resolution. This approach increase the timer interrupt overhead because it is executed more frequently. Sometimes, a more efficient strategy for better timer resolution is to use the ONE_SHOT mode for programming the PIT instead the SQUARE_WAVE mode. In this mode, on each Timer interrupt the PIT is programmed to generate an interrupt at the time of the the next VT expiration time.

- Update MINIX4RT as a branch of MINIX3: MINIX Version 3 offers a lot of improvements over previous versions and MINIX4RT will benefit of it's new features.

- POSIX 1003.1b compatibility: To support standard functions that RTapplications need, such as enhanced IPC, scheduling and memory management control, asynchronous I/O operations and file synchronization. 


\section{References}

[1] Compaq Computer Corporation, "Tru64 UNIX:Guide to Realtime Programming", August 2000, http://www.helsinki.fi/atk/unix/dec_manuals/DOC_51A/HTML/ARH9TBTE/TITLE.HTM.

[2] Tanenbaum Andrew S., Woodhull Albert S., "Sistemas Operativos: Diseño e Implementación 2da Edición", ISBN 9701701658, Editorial Prentice-Hall, 1999.

[3] Paul Ashton, Carl Cerecke,Craig McGeachie, Stuart Yeates, "Use of interaction networks in teaching Minix" Technical Remailbox . COSC 08/95, Dept. of Computer Science . University of Canterbury,1995.

[4] Paul Ashton, Daniel Ayers, Peter Smith. "SunOS MINIX: A tool for use in Operating System laboratories", Technical Remailbox, Australian Computer Science Communications, 16(1): 259-269, 1994.

[5] Stephen J Hartley, "More Experience with MINIX in Operating System lab", available online at ftp://ftp.mcs.drexel.edu/pub/shartley/minix.PO.gz.

[6] Victor Yodaiken, "Cheap Operating System Research and Teaching with Linux", available online at http://citeseer.ist.psu.edu/75556.html.

[7] RTnet-Hard Real-Time Networking for Linux/RTAI, available online at http://www.rts.unihannover.de/rtnet/index.html.

[8] Rether: A Real-Time Ethernet Protocol, http://www.ecsl.cs.sunysb.edu/rether/.

[9] Victor Yodaiken, Michael Barabanov, "A Real-Time Linux", ”, Proceedings of Linux Applications Development and Deployment Conference (USELINUX), January, 1997, available online at http://rtlinux.cs.nmt.edu/.

[10] Takuro Kitayama, Tatsuo Nakajima, and Hideyuki Tokuda, "RT-IPC: An IPC Extension for Real-Time Mach", School of Computer Science, Carnegie Mellon University, Japan Advanced Institute of Science and Technology.

[11] QNX Software Systems Ltd. 2002 - "QNX Neutrino Realtime Operating System - System Architecture", http://www.mikecramer.com/Qnx/momentics_nc_docs/neutrino/sys_arch/kernel.html.

[12] Pablo J. Rogina - Gabriel Wainer., "New Real-Time Extensions to the MINIX operating system", Proc. of 5 th Int. Conference on Information Systems Analysis and Synthesis (ISAS'99),August, 1999.

[13] Gabriel A. Wainer, "Implementing Real-Time services in MINIX", ACM Operating Systems Review, July 1995. 
[14] Mark Russinovich ,"Inside NT's Interrupt Handling”, Windows \& .NET Magazine, November 1997, available on line at http://www.winntmag.com/Articles/Print.cfm?ArticleID=298.

[15] Daniel P. Bovet, Marco Cesati, "Understanding the Linux Kernel Second Edition, 2003" - O'Reilly - 2003.

[16] Keith Loepere, "Mach 3 Kernel Principles", Open Software Foundation and Carnegie Mellon University, July 15, 1992.

[17] Jochen Liedtke, "On $\mu$-Kernel Construction", In Proceedings of the Fifteenth ACM Symposium on Operating System Principles (Copper Mountain Resort, CO., Dec. 3-6). ACM Press, New York, NY, 1995 , pp. $237-250$.

[18] Intel Corporation, Intel Architecture Software Developer's Manual Volume 3: System Programming Guide, 1997.

[19] Kevin Jeffay, Donald L. Stone , “Accounting for Interrupt Handling Costs in Dynamic Priority Task Systems", University of North Carolina at Chapel Hill, Department of Computer Science.

[20] INtime Interrupt Latency Report, "Measured Interrupt Response Times", November, 1998, Technical Paper available on line at http://www.profimatics.de/products/intime/manuals/intime.interrupt.latency.report.pdf.

[21] Daniel I. Katcher, Hiroshi Arakawa, Jay K. Strosnider, "Engineering and Analisys of Fixed Priority Schedulers", Software Engineering, 1993, volume 19, number 9, pages 920-934.

[22] Matthew B. Ragen, "Real-Time Systems With Microsoft Windows NT“, Microsoft Corporation -April 1995, http://www.theragens.com/misc/MR\%20-\%20Windows\%20NT\%20Real-Time.htm.

[23] Herman Bruyninckx , "Real-Time and Embedded Guide”, K.U.Leuven, Mechanical Engineering, Leuven, Belgium.

[24] Real-Time Systems: (Shin) http://cslab.snu.ac.kr/course/rts03/notes/rt_c2mod.ppt.

[25] Gregory Bollella, Kevin Jeffay ,"Support For Real-Time Computing Within General Purpose Operating Systems", 1995.

[26] Victor Yodaiken, "The RTLinux Manifesto", Department of Computer Science New Mexico Institute of Technology.

[27] Victor Yodaiken, "Against Priority Inheritance", Fsmlabs Technical Report, available on line at http://www.fsmlabs.com/articles/inherit/inherit.pdf, June 25, 2002.

[28] “About UNIX and Real-Time Scheduling”, http://www.pcengines.ch/schedule.htm., 1989.

[29] C. L. Liu and J. Layland. "Scheduling algorithms for multiprogramming in a hard real-time environment", Journal of the ACM, 10(1), 1973. 
[30] N. C. Audsley A. Burns M. F. Richardson A. J. Wellings "Hard Real-Time Scheduling: The DeadlineMonotonic Approach", Department of Computer Science, University of York, York, YO1 5DD, England.

[31] J. Stankovic, M. Spuri, K. Ramamritham, and G. Buttazzo, "Deadline Scheduling For Real-Time Systems: EDF and Related Algorithms". Norwell, MA: Kluwer, 1998.

[32] Aloysius Ka-Lau Mok. "Fundamental Design Problems of Distributed Systems for the Hard Real-Time Environment." MIT LCS Technical Report 297, Department of Computer Science, MIT, May, 1983.

[33] TICS Realtime, "Different Timing Mechanisms and How They are Used", available on line at http://www.concentric.net/ Tics/tics1196a.htm.

[34] George Varghese, Tony Lauck, "Hashed and Hierarchical Timing Wheels: Efficient Data Structures for Implementing a Timer Facility", IEEE $\backslash$ slash ACM Transactions on Networking. (1996), available on line at http://citeseer.nj.nec.com/cache/papers/cs/1218/http:zSzzSzwww.ccrc.wustl.eduzSz varghesezSzPAPERSzSzt wheel.pdf/varghese96hashed.pdf.

[35] Robert Hill, Balaji Srinivasan, Shyam Pather, Douglas Niehaus, "Temporal Resolution and Real-Time Extensions to Linux.", ITTC Technical Report ITTC-FY98-11510-03, University of Kansas, 1998.

[36] Jochen Liedtke, "Improving IPC by Kernel Design", 14th ACM Symposium on Operating System Principles (SOSP) 5th-8th December, 1993, Asheville, North Carolina.

[37] Mark W. Borger, Ragunathan Rajkumar. "Implementing Priority Inheritance Algorithms in an Ada Runtime System", Technical Remailbox . CMU/SEI-89-TR-15. ESD-TR-89-23. April 1989. Software Engineering Institute Carnegie Mellon University.

[38] Sha, L., Lehoczky, J.P., and Rajkumar, R. "Priority Inheritance Protocols: An Approach to Real-Time Synchronization". Tech. Rept. CMU-CS-87-181, Carnegie Mellon University, Computer Science Department, 1987.

[39] Bruno Dutertre, “The Priority Ceiling Protocol: Formalization and Analysis Using PVS”, System Design Laboratory SRI International, Menlo Park, CA 94025, November 8, 1999. 


\section{Appendix A: RT-SYSTEM CALLS AND RT-KERNEL CALLS REFERENCE}

\section{A.1. System Calls Reference}

\section{A.1.1. mrt_RTstart}

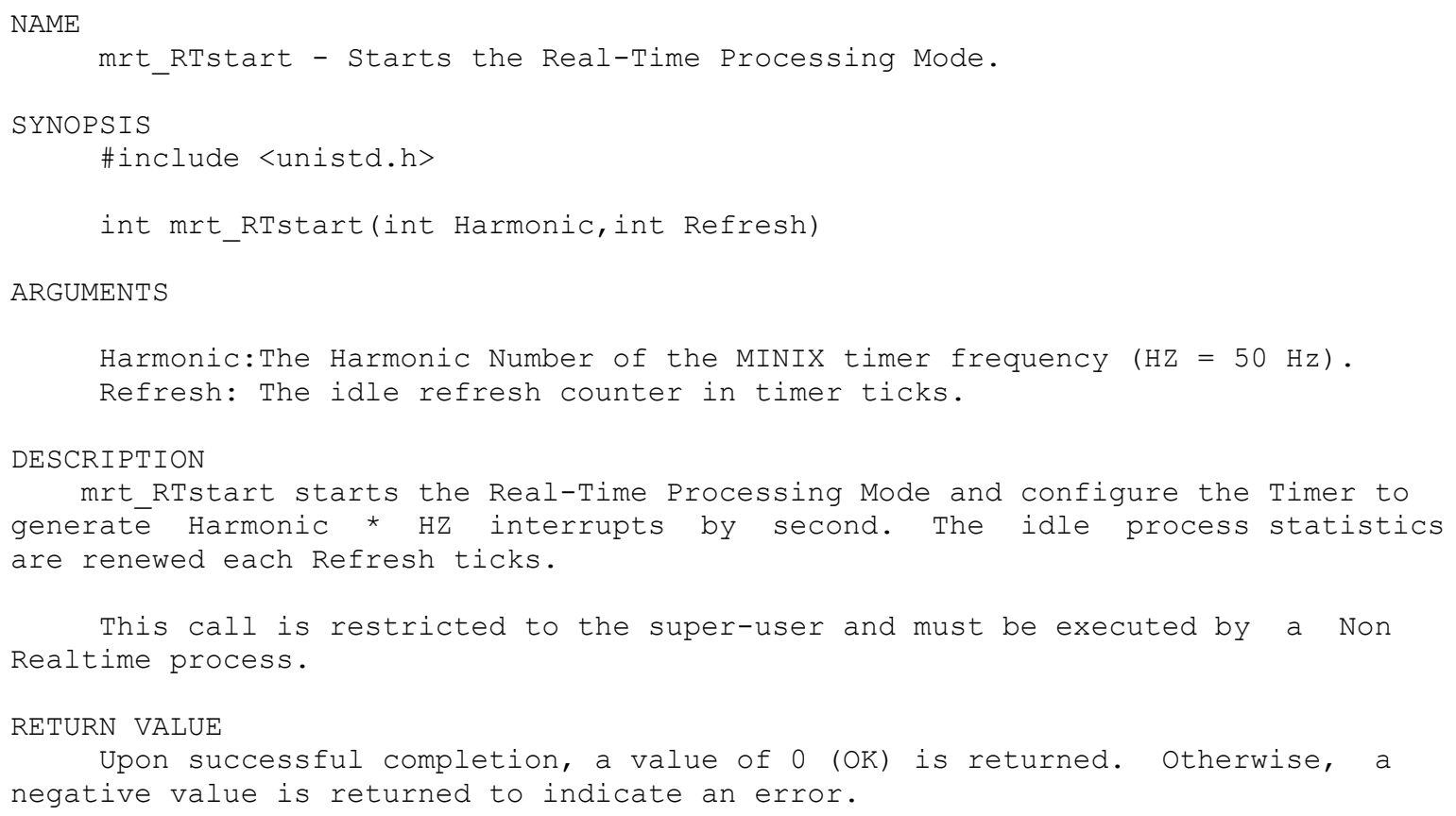


ERRORS

mrt RTstart will fail and processing mode will be unchanged if one or more of the following are true:

[E_MRT_BADHARM]A bad value has been specified for the Harmonic argument.

[E_MRT_BADRFSH]A bad value has been specified for the Refresh argument.

[E_MRT_RTACTIVE]The system is already in Real-Time Processing Mode.

SEE ALSO

mrt_RTstop (2).

\section{A.1.2. mrt_RTstop}

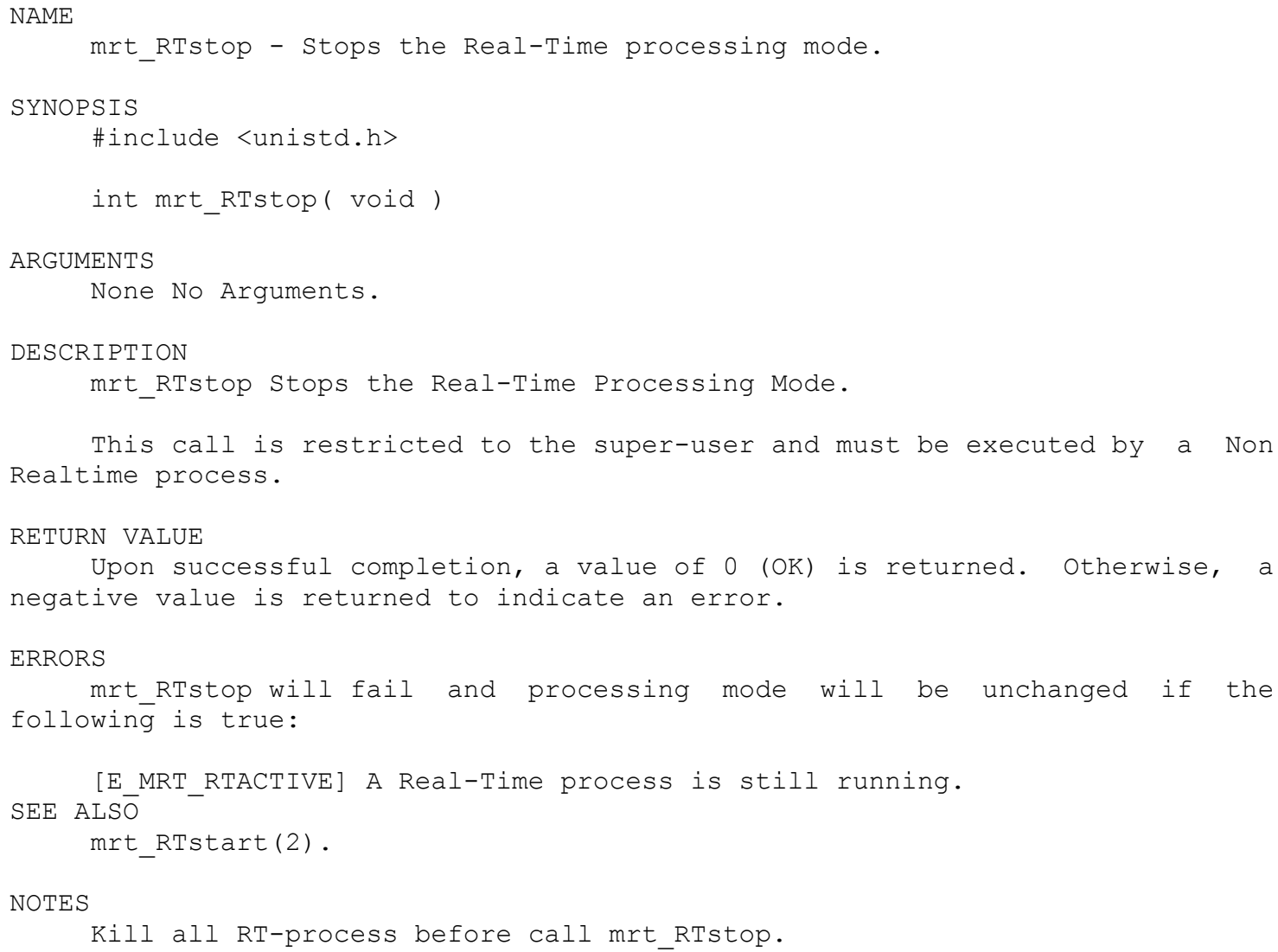

\section{A.1.3. mrt_clrpstat}

NAME

mrt_clrpstat - Clears all RT-processing statistics of a specified process. SYNOPSIS

\#include <unistd.h>

int mrt_clrpstat (pid_t Pid)

ARGUMENTS

Pid: The PID number of the RT-process. 
DESCRIPTION

mrt clrpstat Clears all RT-processing statistics of a specified process. This call is restricted to the super-user. It must be executed by a NRT-process.

RETURN VALUE

Upon successful completion, a value of 0 (OK) is returned. Otherwise, a negative value is returned to indicate an error.

ERRORS

mrt_clrpstat will fail if one or more of the following are true:

[E_BAD_PROC]The RT-process of the specified Pid does not exist.

SEE ALSO

mrt_getpstat (2).

\section{A.1.4. mrt_getiattr}

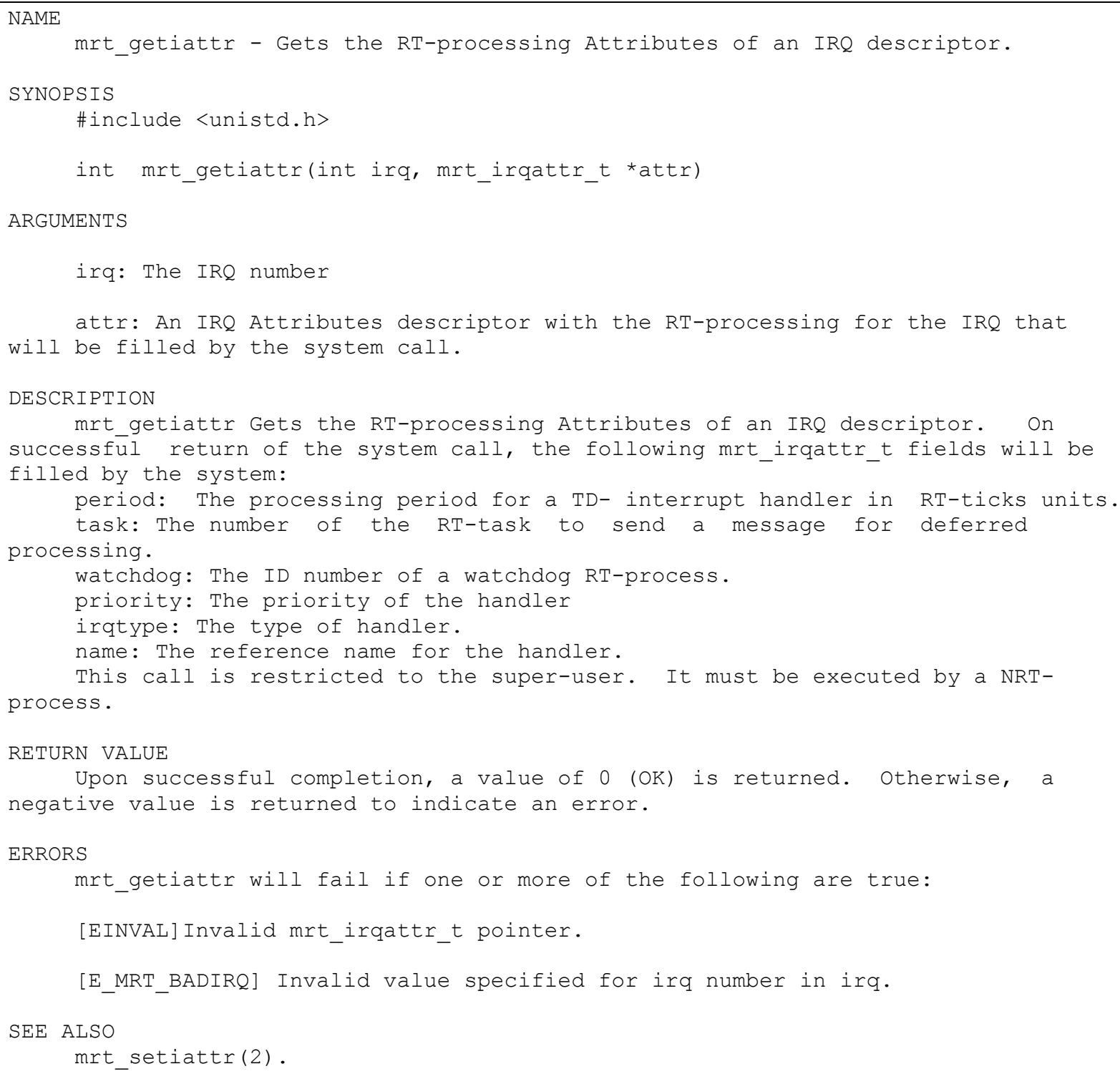




\section{A.1.5. mrt getiint}

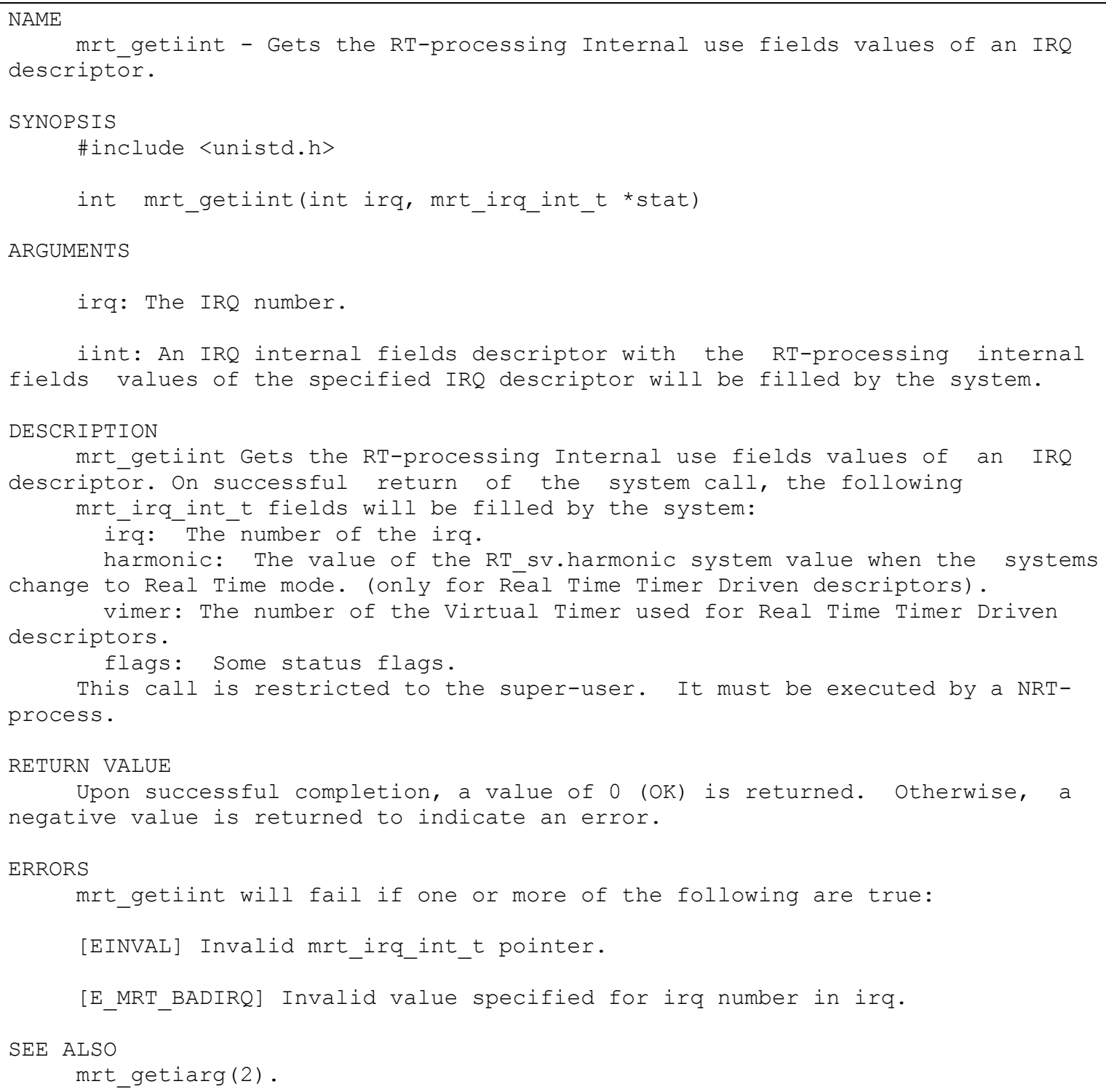

Upon successful completion, a value of 0 (OK) is returned. Otherwise, a negative value is returned to indicate an error.

\section{A.1.6. mrt_getistat}

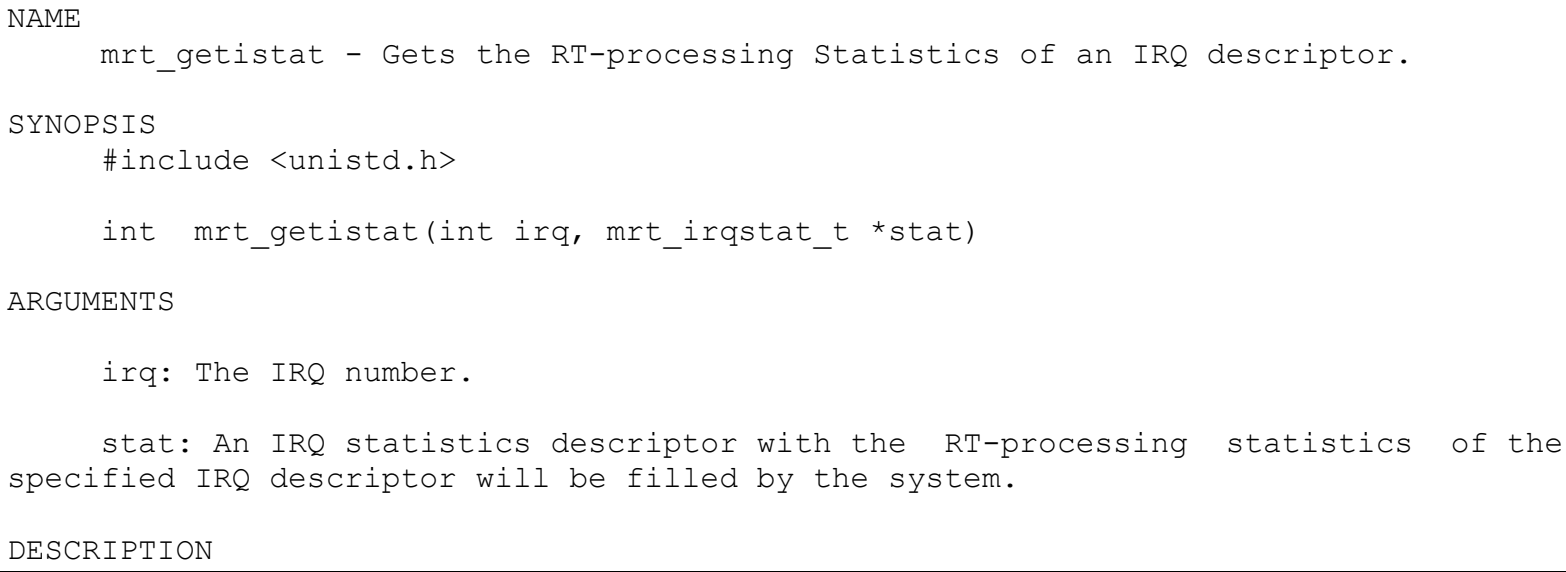


mrt_getistat Gets the RT-processing statistics of an IRQ descriptor. On successful return of the system call, the following mrt_irqstat_t fields will be filled by the system:

count: An interrupt counter.

maxrain: The maximun number of interrupts into a period of Timer

Driven handler.

maxrain: The number of Missed DeadLines of the handler.

timestamp: The last interrupt timestamp.

maxlat: The maximun (approximate) latency of the handler. process.

This call is restricted to the super-user. It must be executed by a NRT-

RETURN VALUE

Upon successful completion, a value of 0 (OK) is returned. Otherwise, a negative value is returned to indicate an error.

ERRORS

mrt_getistat will fail if one or more of the following are true:

[EINVAL] Invalid mrt_irqstat_t pointer.

[E_MRT_BADIRQ] Invalid specified irq number.

SEE ALSO

mrt_getiattr(2).

\section{A.1.7. mrt_getpattr}

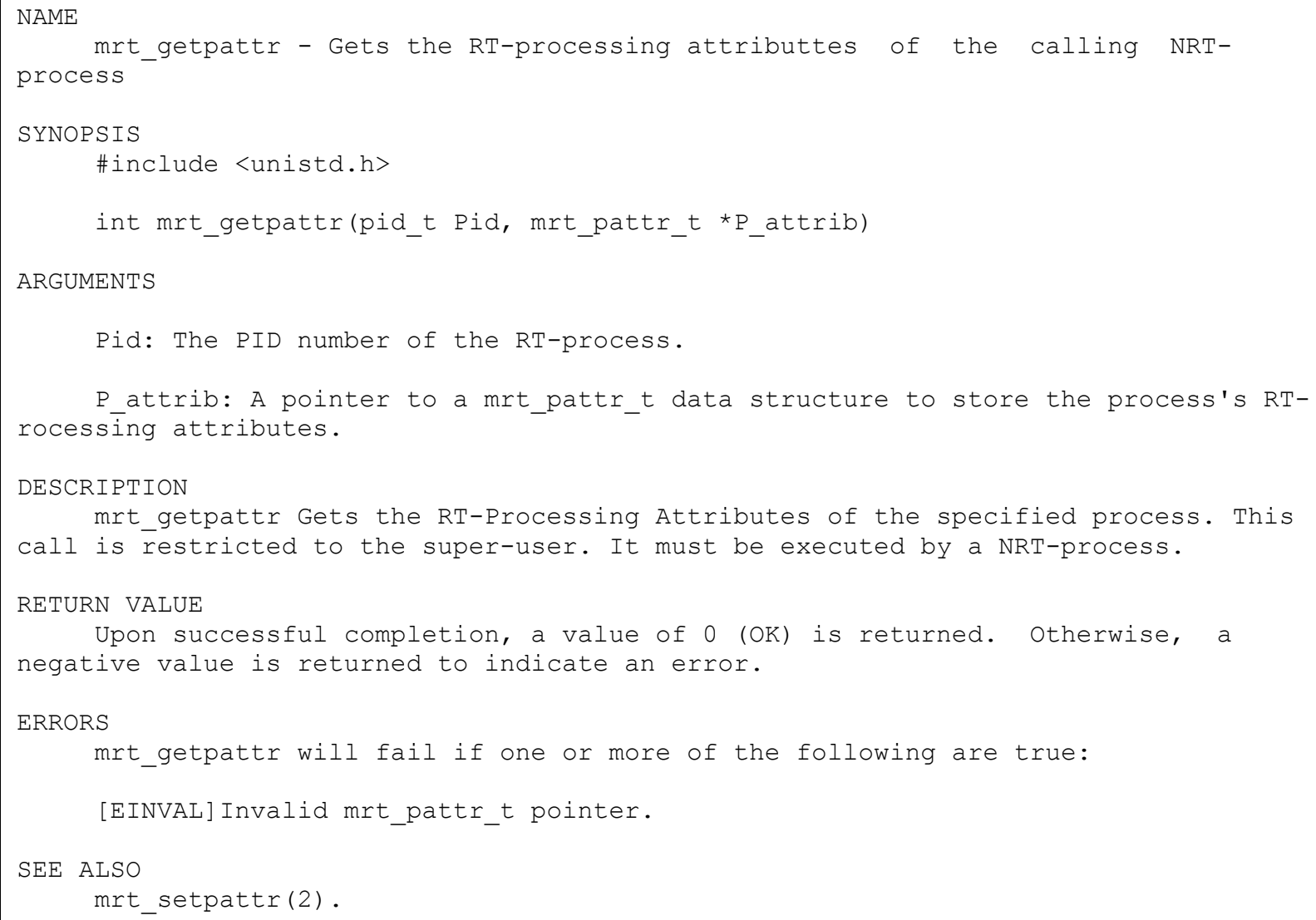

Pid: The PID number of the RT-process.

P_attrib: A pointer to a mrt_pattr_t data structure to store the process's RTrocessing attributes.

DESCRIPTION

mrt_getpattr Gets the RT-Processing Attributes of the specified process. This call is restricted to the super-user. It must be executed by a NRT-process.

RETURN VALUE

Upon successful completion, a value of 0 (OK) is returned. Otherwise, a negative value is returned to indicate an error.

ERRORS

mrt_getpattr will fail if one or more of the following are true:

[EINVAL] Invalid mrt_pattr_t pointer.

SEE ALSO

mrt_setpattr(2). 


\section{A.1.8. mrt_getpint}

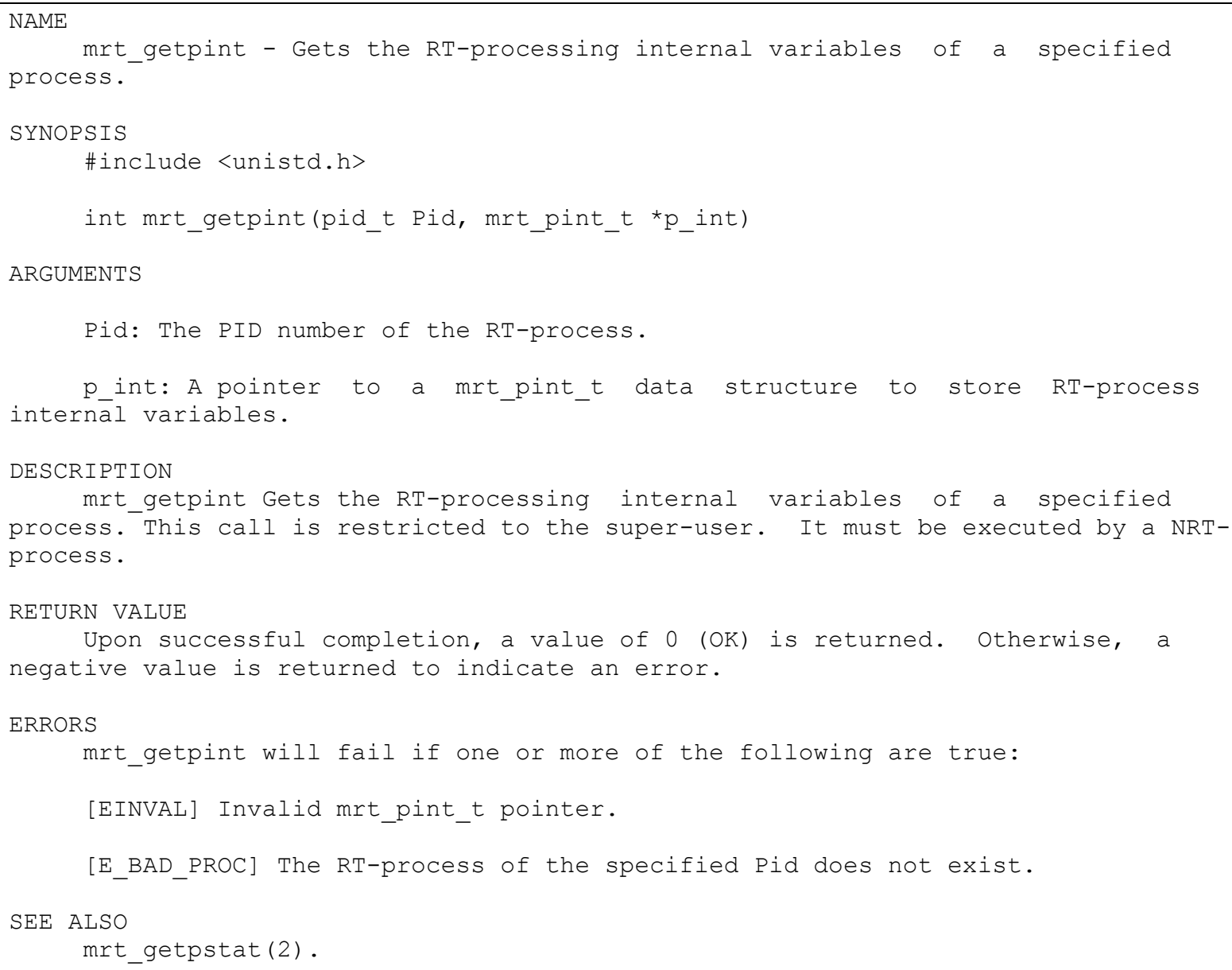

\section{A.1.9. mrt getpstat}

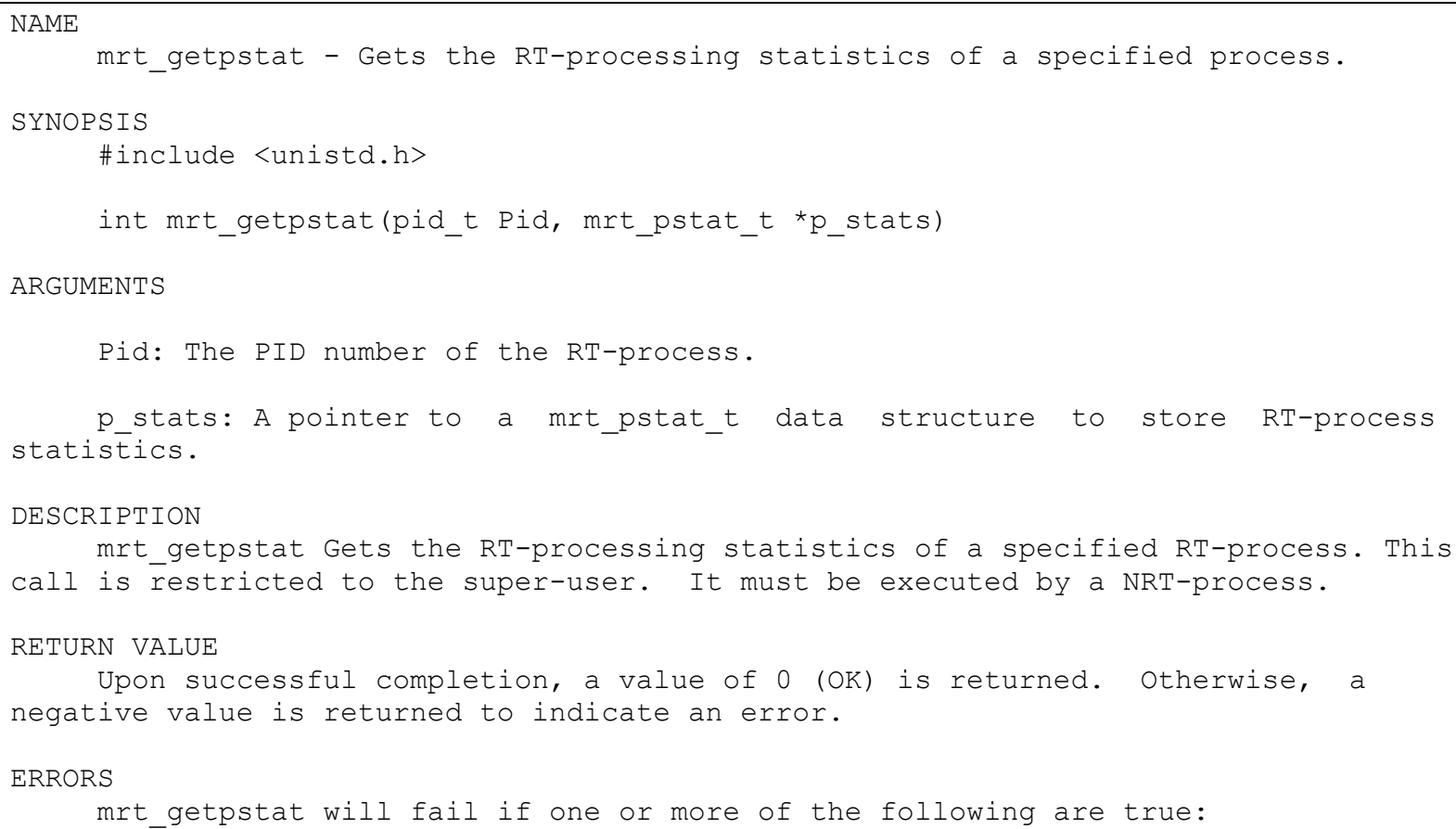


[EINVAL] Invalid mrt_pstat_t pointer.

[E_BAD_PROC] The RT-process of the specified Pid does not exist.

SEE ALSO

mrt_getpint(2). mrt_clrpstat (2).

\section{A.1.10.mrt_getsstat}

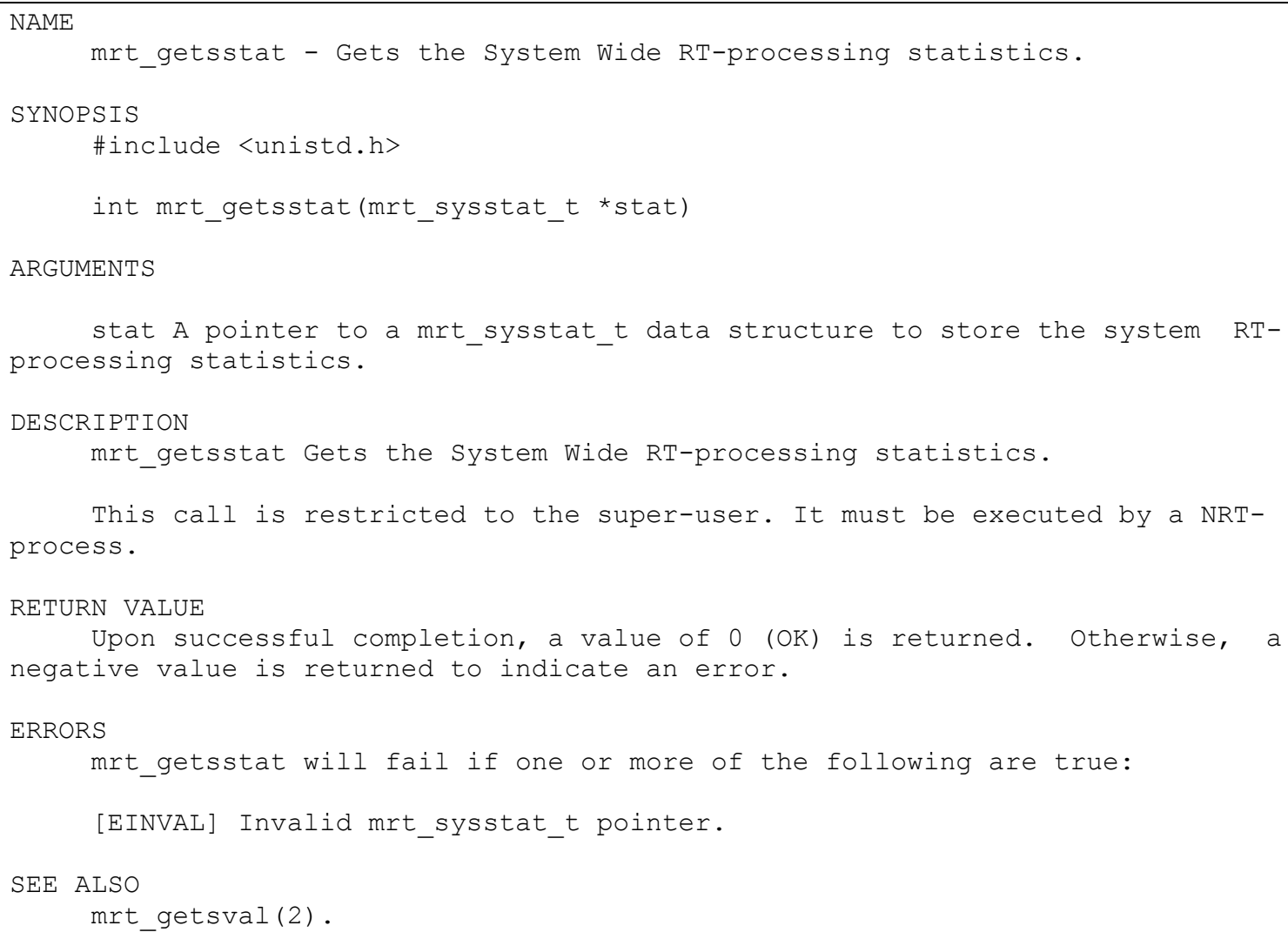

\section{A.1.11.mrt_getsval}

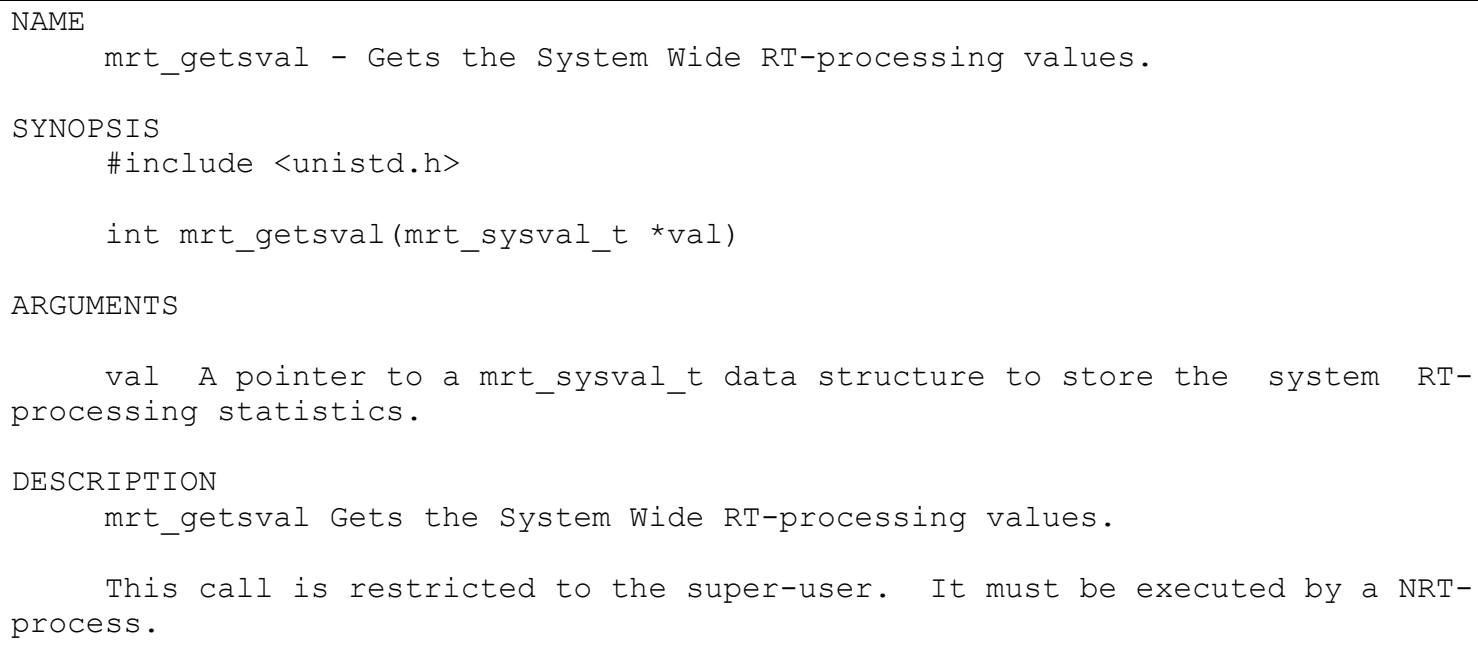


RETURN VALUE

Upon successful completion, a value of 0 (OK) is returned. Otherwise, a negative value is returned to indicate an error.

ERRORS

mrt_getsval will fail if one or more of the following are true:

[EINVAL] Invalid mrt_sysval_t pointer.

SEE ALSO

mrt_getsstat (2).

\section{A.1.12.mrt_restart}

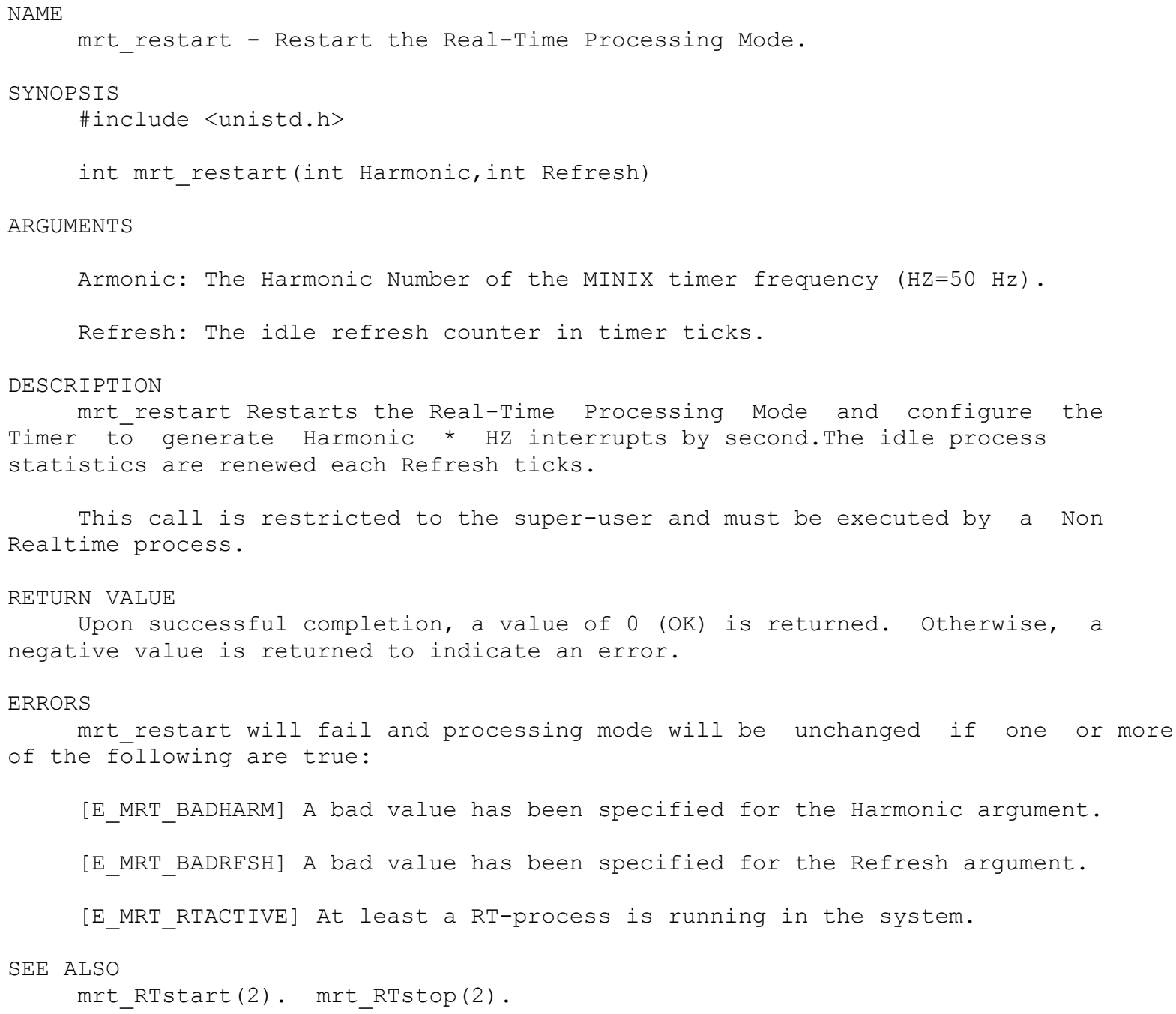

\section{A.1.13.mrt_setiattr}

NAME mrt_setiattr - Sets the RT-processing Attributes of an IRQ descriptor. SYNOPSIS

\#include <unistd.h> 


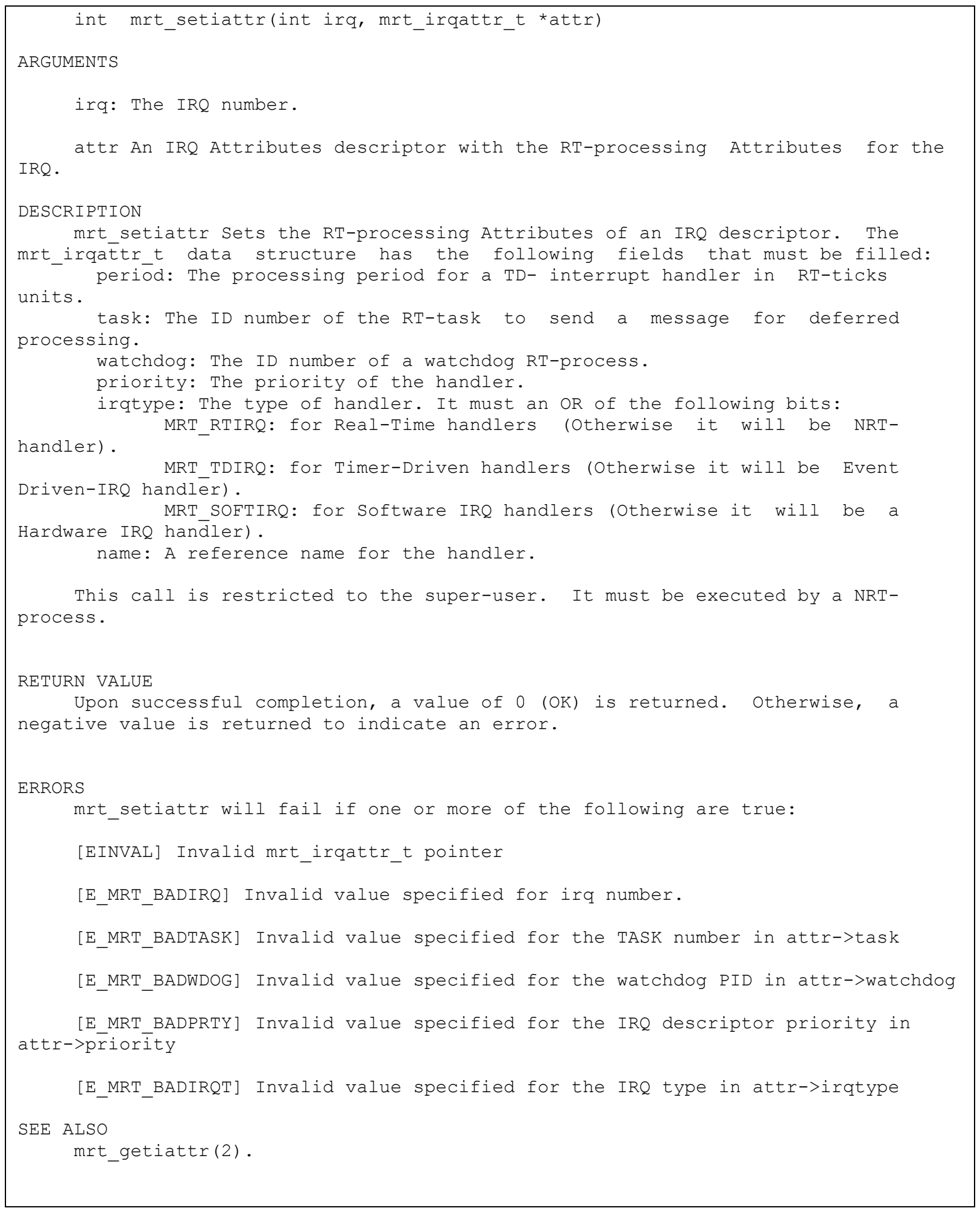

\section{A.1.14.mrt_setpattr}

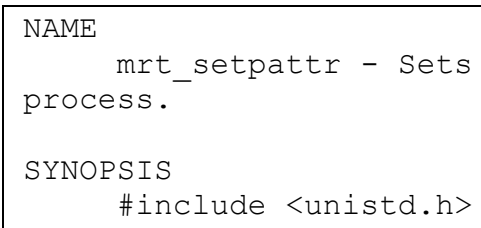


int mrt_setpattr(mrt_pattr_t ${ }^{\star}$ p_attrib)

ARGUMENTS

p_attrib: A pointer to a mrt_pattr_t data structure with the RT-processing attributes of the calling NRT-process. The field of the data structure are: flags: Real Time Flags.

baseprty: Real Time Base priority.

period: period in RT-ticks for Periodic Processes.

limit: maximun number of process schedulings.

deadline: process deadline.

watchdog: Watchdog process.

mq_size: Message Queue Size.

mq_flags: Message Queue Policy Flags.

The Real Time Flags flags can be an OR of the following flags:

MRT_P_REALTIME: to set the process as Real-Time.

MRT_P_PERIODIC: to set the process as Real-Time Periodic.

flags :

The Message Queue Policy Flags mq_flags can be an or of the following

MRT_PRTYORDER: Priority Order Policy (otherwise FIFO policy).

MRT_PRTYINHERIT: Priority Inheritance policy.

\section{DESCRIPTION}

mrt_setpattr sets the RT-Processing Attributes of the calling NRT-process.

This cal $\bar{l}$ is restricted to the super-user. It must be executed by a NRT-process in System Real Time Processing Mode.

RETURN VALUE

Upon successful completion, a value of 0 (OK) is returned. Otherwise, a negative value is returned to indicate an error.

ERRORS

mrt_setpattr will fail if one or more of the following are true:

[EINVAL] Invalid mrt_pattr_t pointer.

[E_MRT_BADPTYPE] Invalid value specified for process type in p_attrib->ptype.

[E_MRT_BADPRTY]Invalid value specified for process priority in p_attrib->priority.

[E_MRT_BADWDOG] Non existing or NRT-watchdog process specified in p_attrib $->$ watch $\bar{d}$ og.

[E MRT NOMSGQ] The system cannot assign a Message Queue of the specified size in p_attrib->mq_size.

[E_MRT_NOVTIMER] The system cannot assign a Virtual Timer for a Periodic RT-process.

SEE ALSO

mrt_getpattr(2). 


\section{A.2. Kernel Calls Reference}

\section{A.2.1. mrt_rqst}

NAME

mrt_rqst - sends a synchronous request message.

SYNOPSIS

\#include <unistd.h>

int mrt_rqst (mrtpid_t mrtpid, mrt_msg_t*m_ptr, lcounter_t timeout);

ARGUMENTS

mrtpid: The RT-PID of the destination RT-process.

m_ptr: A pointer to the message buffer.

timeout: The number of RT-ticks for waiting to send de request. A MRT NOWAIT value can be specified to return without waiting if the destination process is not waiting for this message. A MRT FOREVER value can be specified to wait until the destination process receive the message.

DESCRIPTION

mrt rqst sends a request message to a process through a message queue in a synchronous way with or without specifying a timeout. It must be executed by a RTprocess.

RETURN VALUE

Upon successful completion, a value of 0 (OK) is returned. Otherwise, a negative value is returned to indicate an error.

ERRORS

mrt_rqst will fail if one or more of the following are true:

[E_MRT_NORTMODE] The system is in Non Real Time processing Mode.

[E_MRT_BADPROC] The process PID does not much with the process number use by the kernel.

[E_MRT_BADPTYPE] The Destination process is not a RT-process.

[E BAD DEST] The Destination process is does not exist.

[E_TRY_AGAIN] The Destination process message queue is full.

[E_MRT_NOMQENT] The system message queue entry free pool is empty.

SEE ALSO

mrt arqst(2). mrt uprqst(2) .

\section{A.2.2. mrt_arqst}

NAME

mrt_arqst - sends an Asynchronous request message.

SYNOPSIS

\#include <unistd.h> 
int mrt_arqst(mrtpid_t mrtpid, mrt_msg_t *m_ptr);

ARGUMENTS

mrtpid: The RT-PID of the destination RT-process.

m_ptr: A pointer to the message buffer.

DESCRIPTION

mrt arqst sends a request message to a process through a message queue in an Asynchronous way. It must be executed by a RT-process.

RETURN VALUE

Upon successful completion, a value of 0 (OK) is returned. Otherwise, a negative value is returned to indicate an error.

ERRORS

mrt_arqst will fail if one or more of the following are true:

[E_MRT_NORTMODE] The system is in Non Real Time processing Mode.

[E_MRT_BADPTYPE] The Source process is not a RT-process.

[E_MRT_BADPTYPE] The Destination process is not a RT-process.

[E_BAD_DEST] The Destination process is does not exist.

[E_TRY_AGAIN] The Destination process message queue is full.

[E_MRT_NOMQENT] The system message queue entry free pool is empty.

SEE ALSO

mrt_rqst (2). mrt_uprqst (2).

\section{A.2.3. mrt_uprqst}

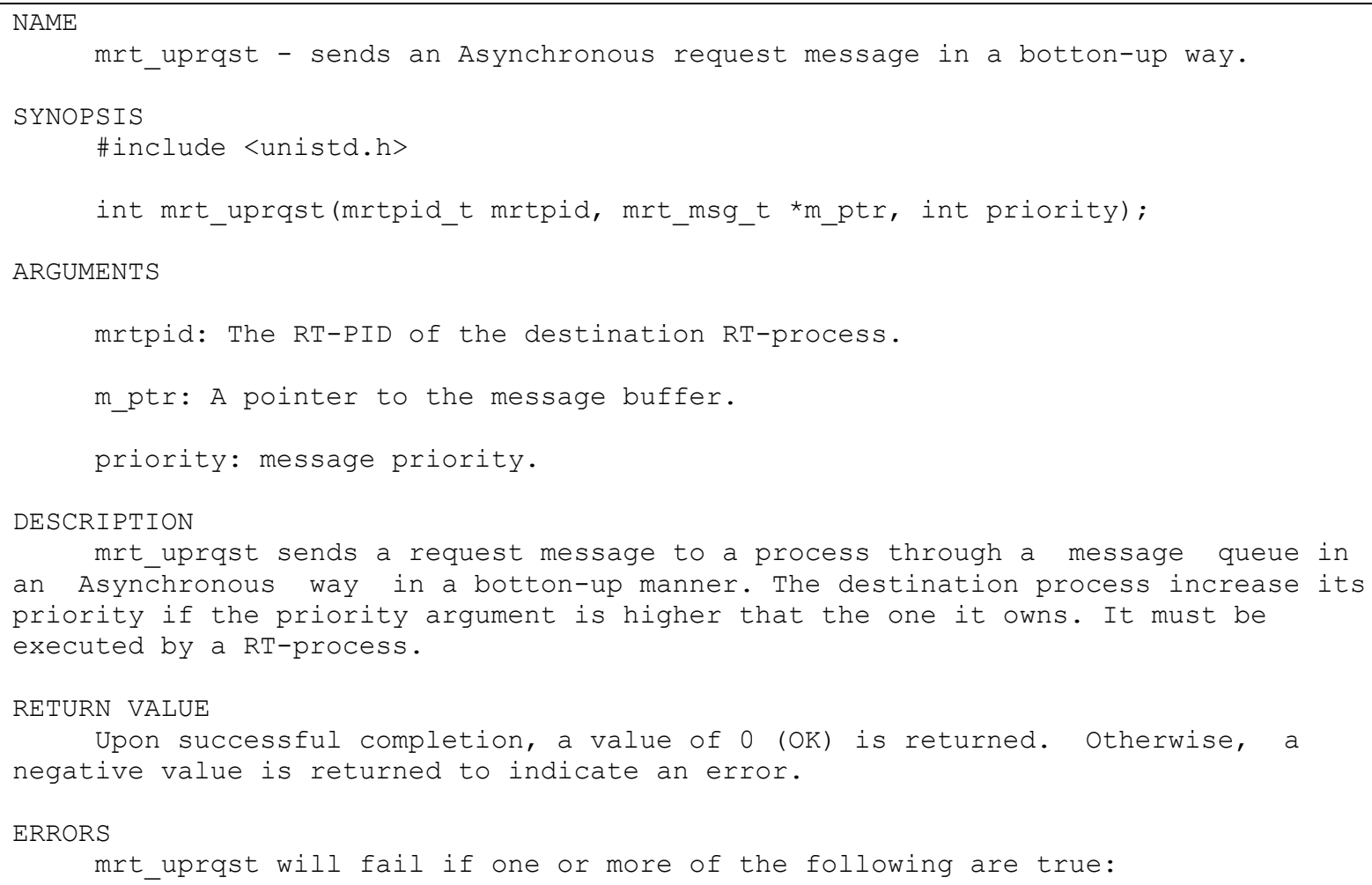




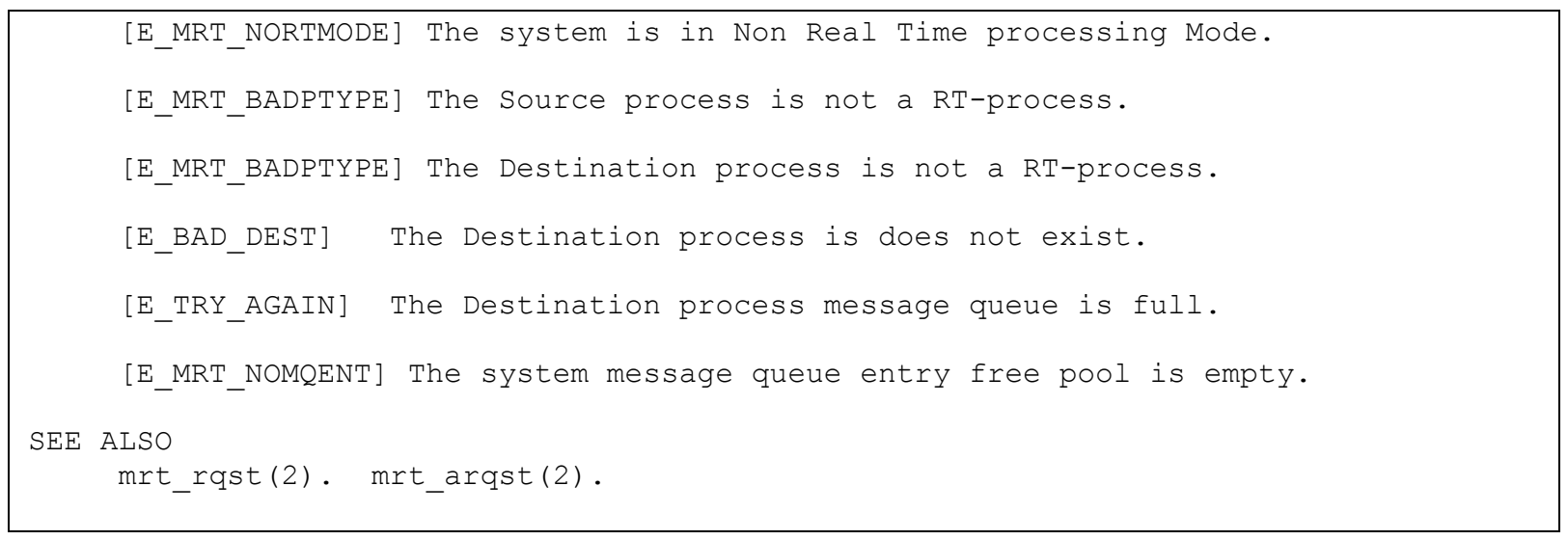

\section{A.2.4. mrt_sign}

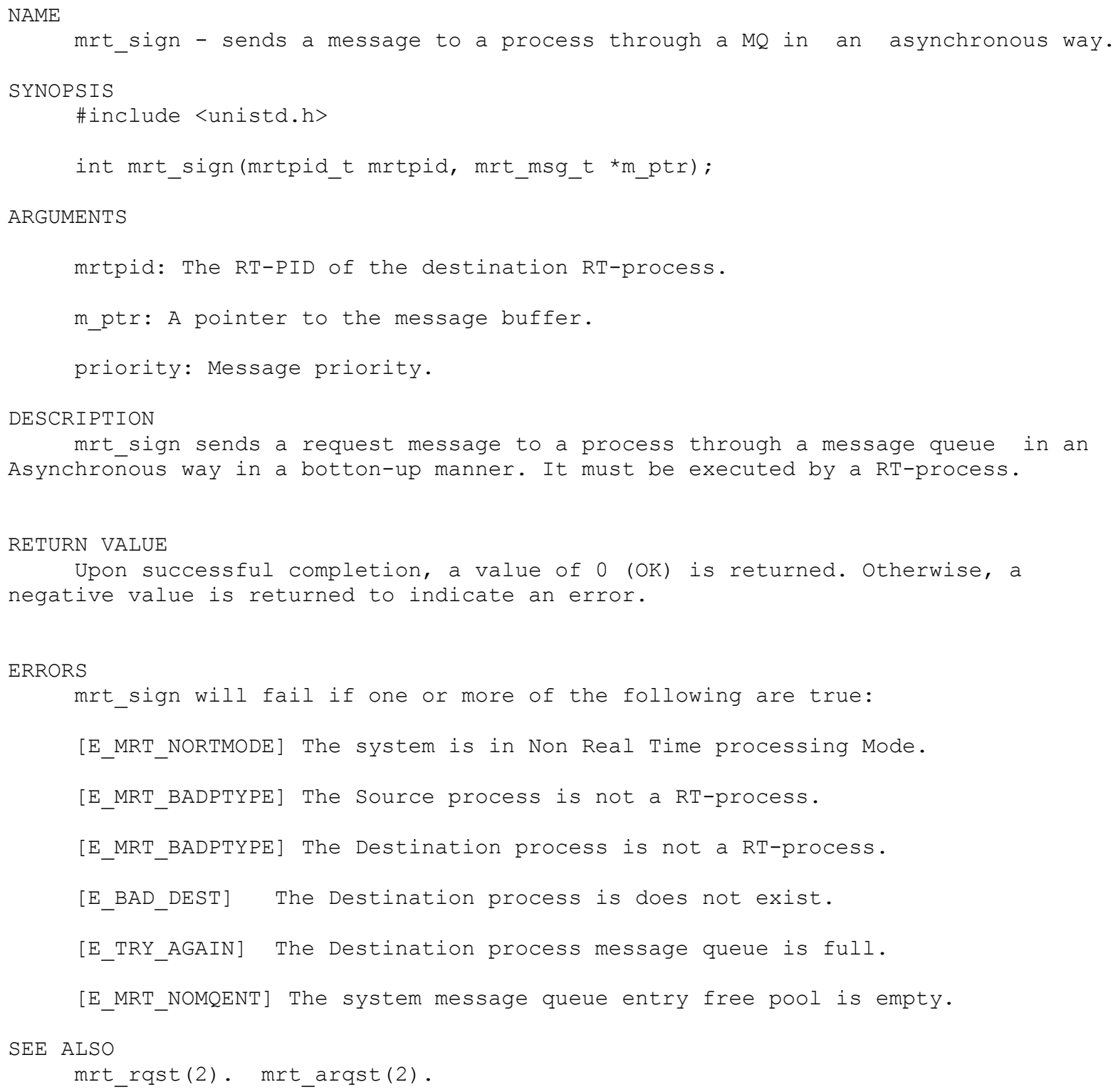




\section{A.2.5. mrt_reply}

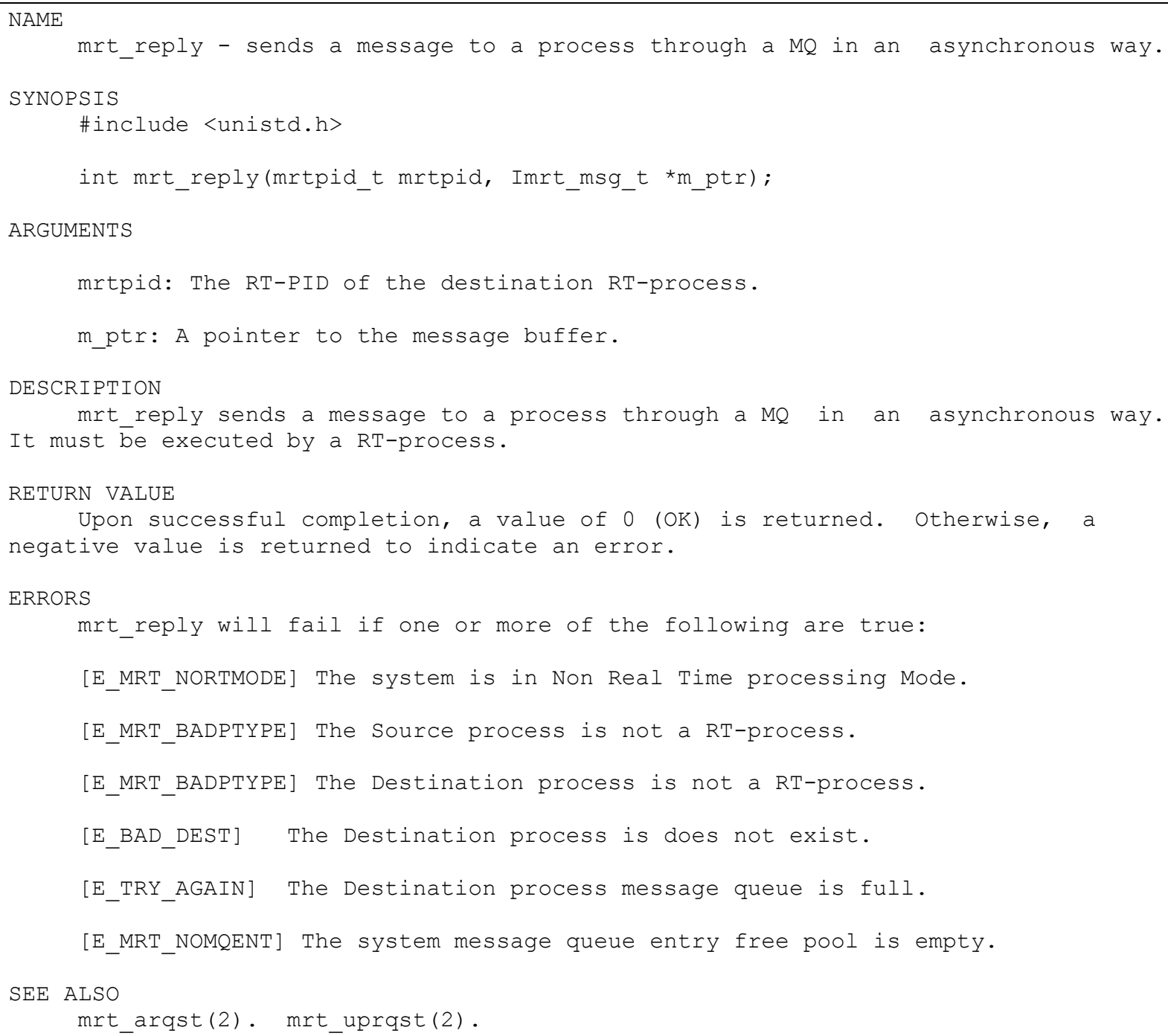

A.2.6. mrt_rev

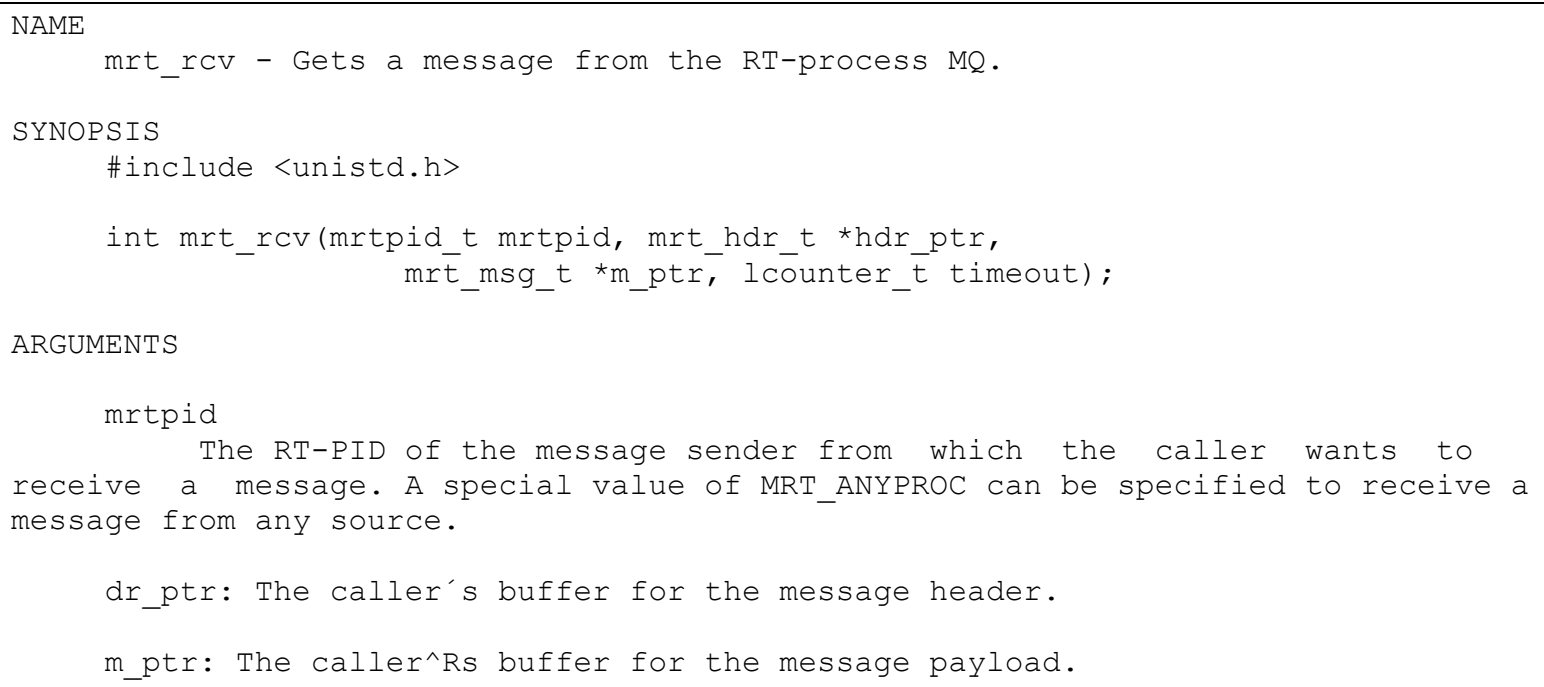


timeout: The number of RT-ticks for waiting to receive a message. A MRT NOWAIT value can be specified to return with o without receiving the message. If the message has been received, the function return code is oK else returns E_TRY_AGAIN. A MRT_FOREVER value can be specified to waits until the message is réceive.

DESCRIPTION

mrt_rcv gets a message from the RT-process MQ. It must be executed by a RTprocess.

RETURN VALUE

Upon successful completion, a value of 0 (OK) is returned. Otherwise, a negative value is returned to indicate an error.

ERRORS

mrt_rcv will fail if one or more of the following are true:

[E_MRT_NORTMODE] The system is in Non Real Time processing Mode.

[E_MRT_BADPTYPE] The Source process is not a RT-process.

[E TRY AGAIN] The process has specified a MRT NOWAIT timeout and the MQ is empty or there are not any message from the specified source.

[MRT_NOVTIMER] The process has specified a timeout to wait for a message but there are not any Virtual Timer to allocate.

SEE ALSO

mrt_rqst(2). mrt_reply(2).

\section{A.2.7. mrt_sleep}

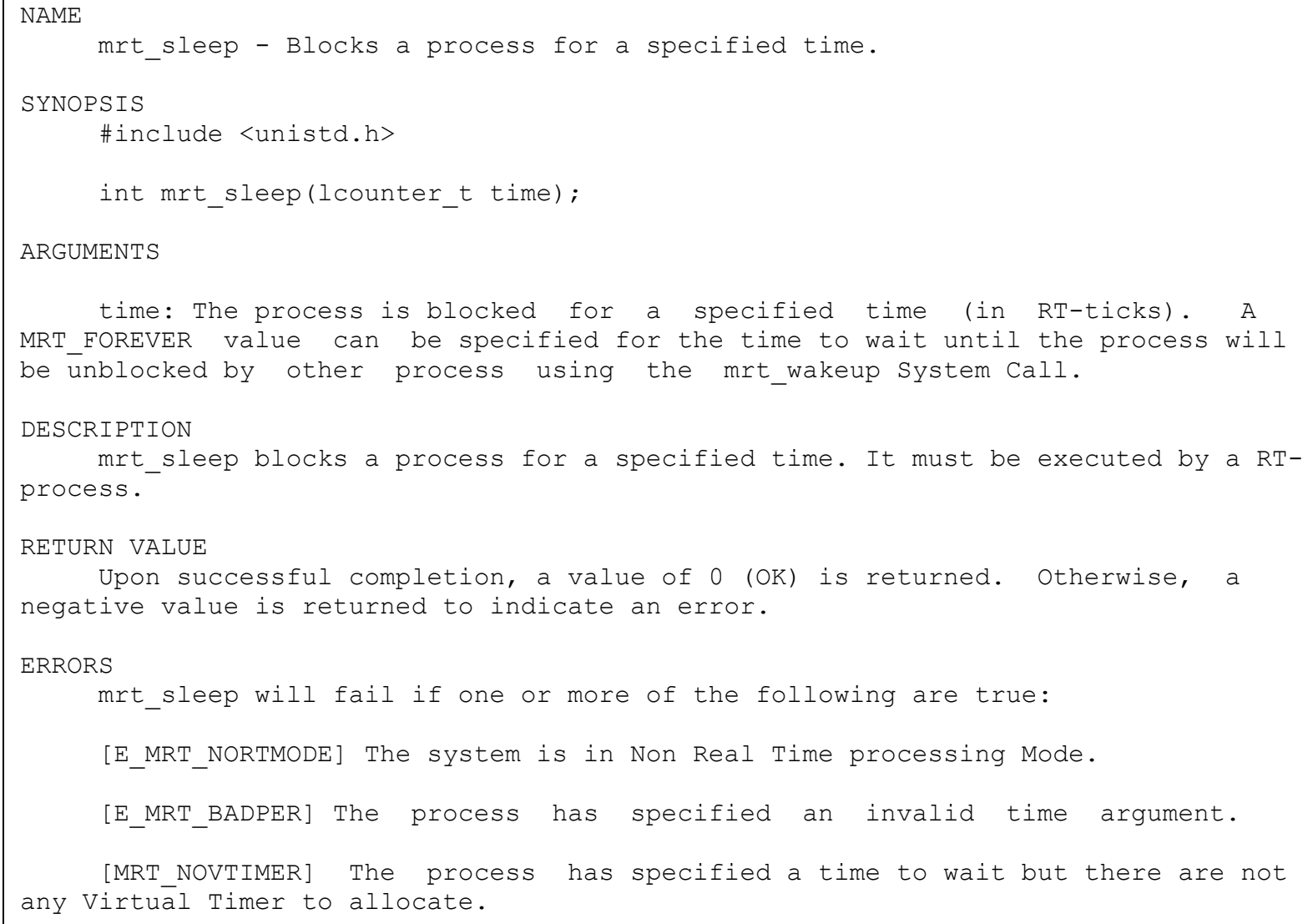

ARGUMENTS

time: The process is blocked for a specified time (in RT-ticks). A MRT_FOREVER value can be specified for the time to wait until the process will be unblocked by other process using the mrt_wakeup system Call.

DESCRIPTION

mrt_sleep blocks a process for a specified time. It must be executed by a RTprocess.

RETURN VALUE

Upon successful completion, a value of 0 (OK) is returned. Otherwise, a negative value is returned to indicate an error.

ERRORS

mrt_sleep will fail if one or more of the following are true:

[E_MRT_NORTMODE] The system is in Non Real Time processing Mode.

[E_MRT_BADPER] The process has specified an invalid time argument.

[MRT_NOVTIMER] The process has specified a time to wait but there are not any Virtuā Timer to allocate. 
SEE ALSO

mrt_wakeup (2).

\section{A.2.8. mrt_wakeup}

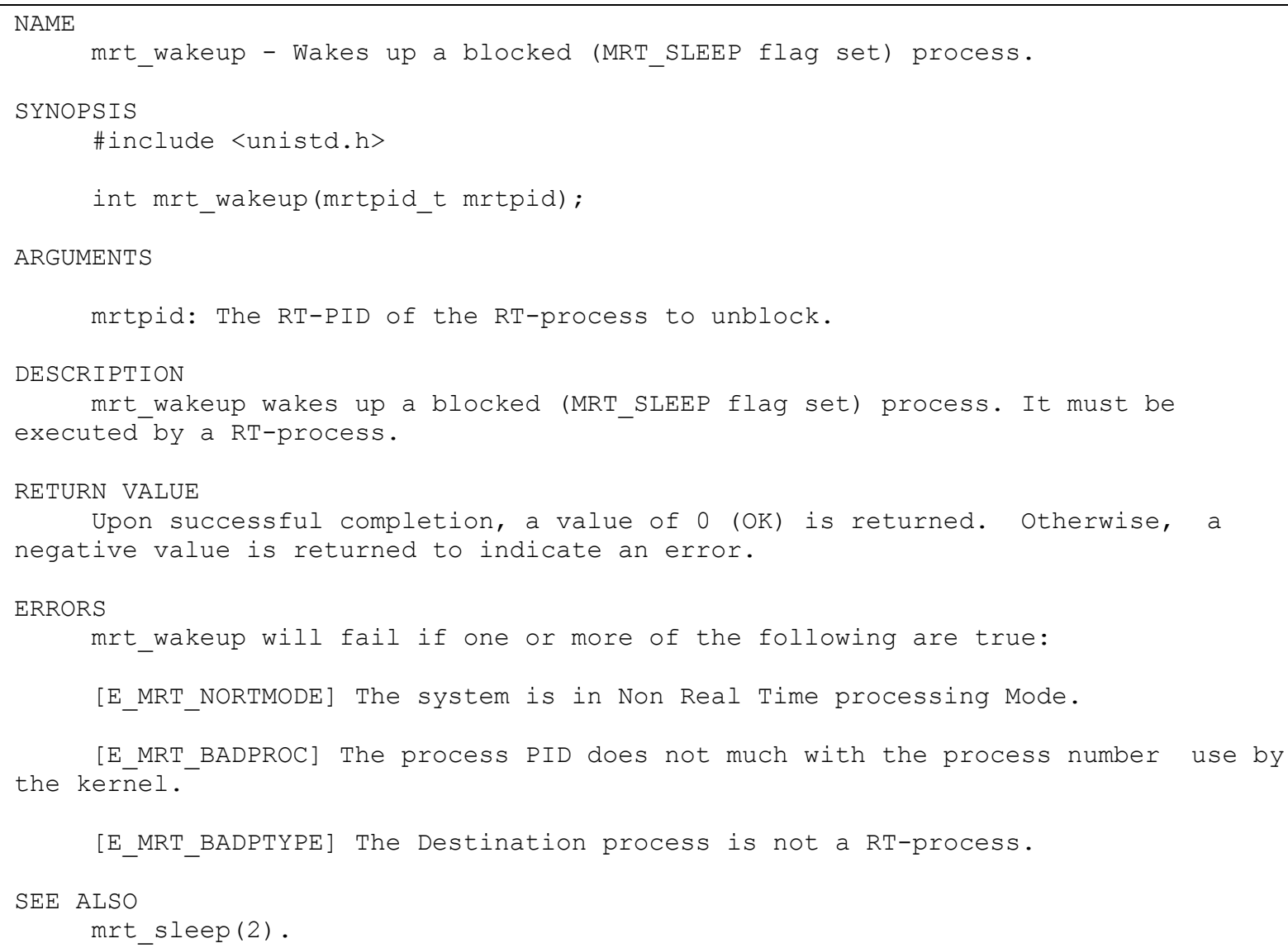

\section{A.2.9. prt_print}

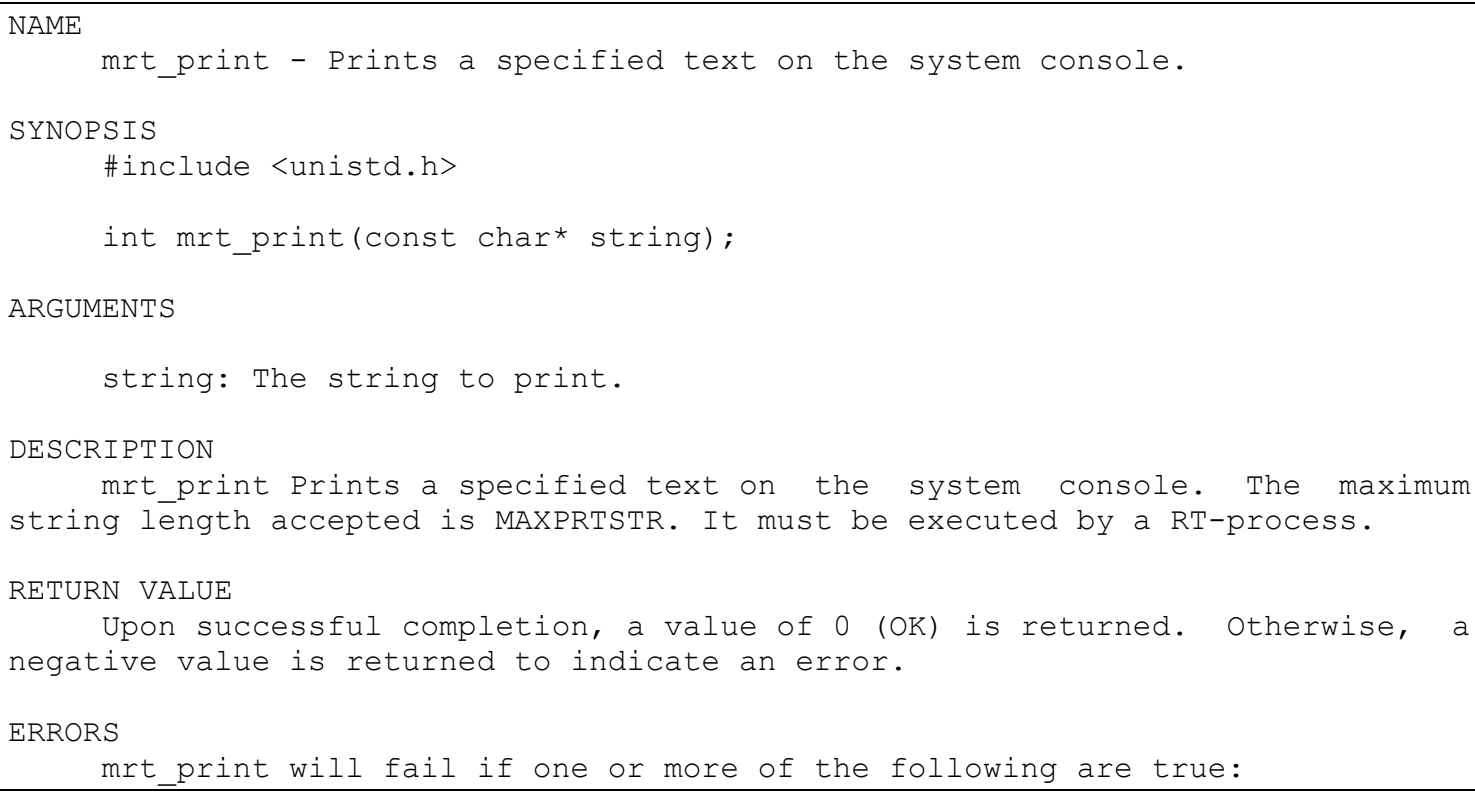


[E_MRT_NORTMODE] The system is in Non Real Time processing Mode.

[E_MRT_BADPTYPE] The caller process is not a RT-process. 


\section{Appendix B: SAMPLE PROGRAMS}

This appendix show several sample programs that use MINIX4RT System Calls and Kernel Calls.

\section{B.1. mrtstart.c}

This program starts the Real-Time processing mode. The harmonic frequency of MINIX Timer can be specified as an argument and the number of Timer ticks to refresh IDLE process statistics. By default harmonic $=4$ and refresh $=200$ are assumed.

\section{Listing B.1: mrtstart.c}

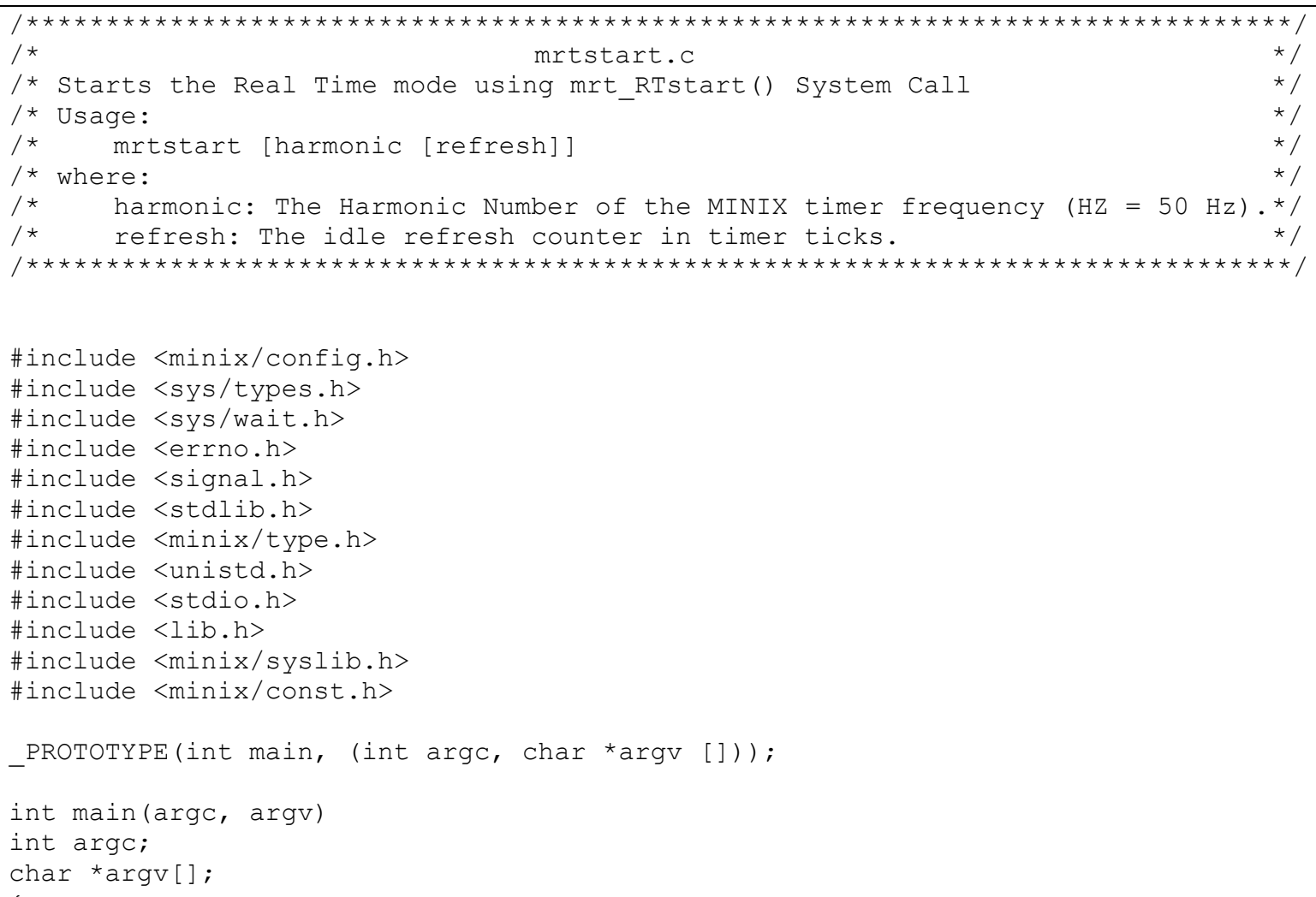




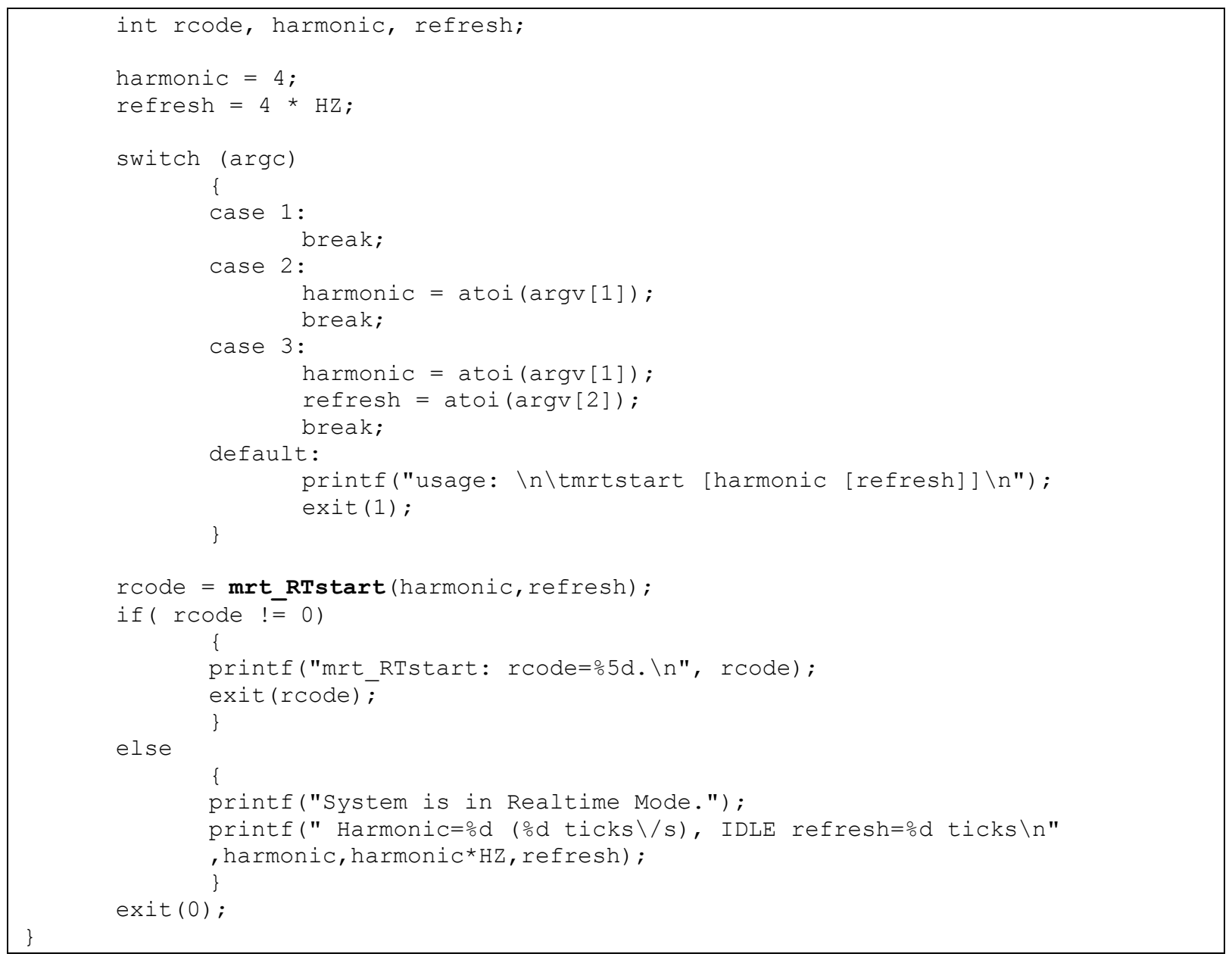

\section{B.2. mrtstop.c}

This program stops the Real-Time processing mode only if there are not any RT-process running in the system.

\section{Listing B.2: mrtstop.c}

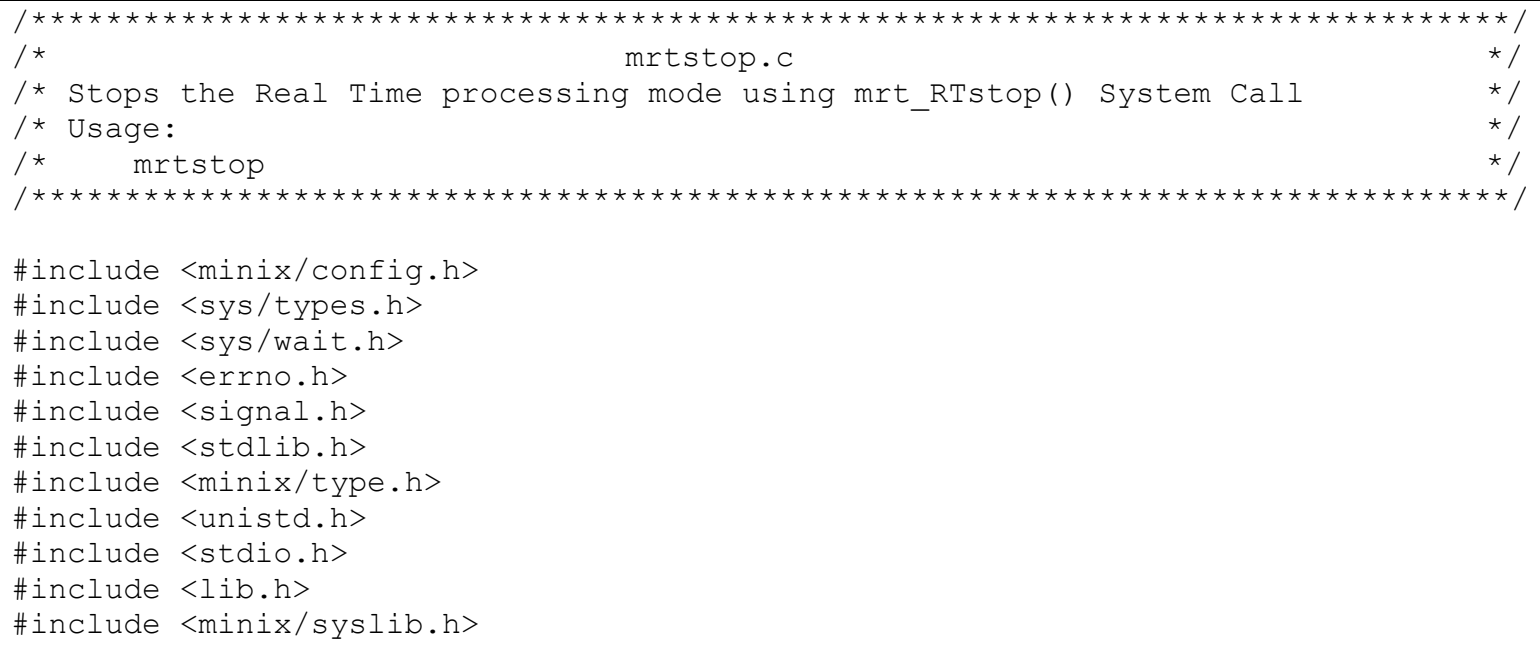




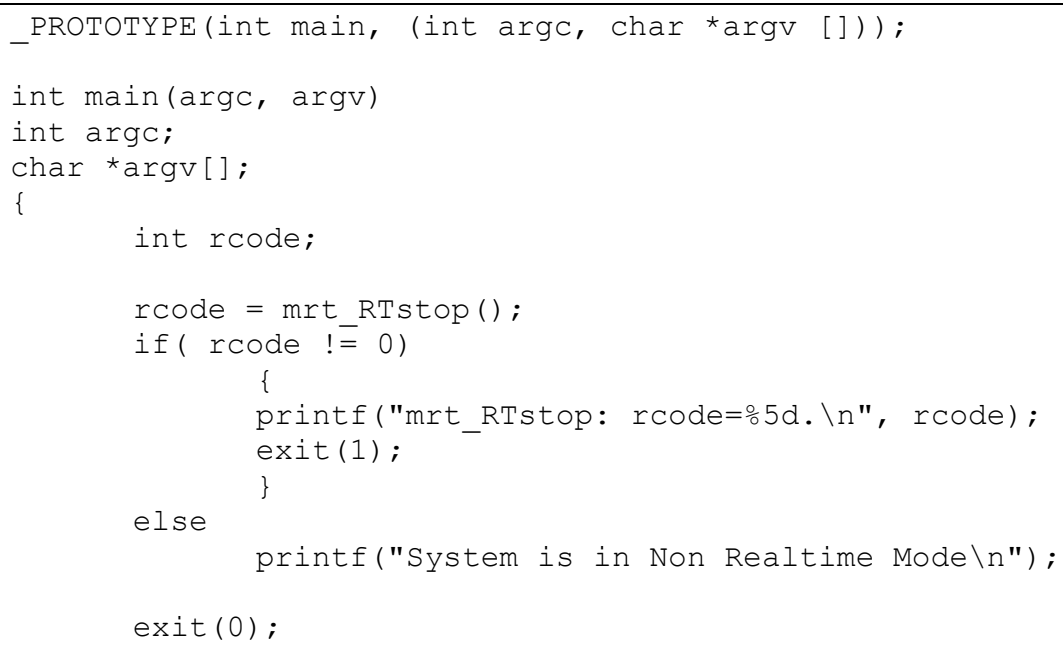

\section{B.3. mrttest1.c}

This program gets and displays Interrrupt Descriptors of attribute fields. At the last line the test must display $\operatorname{irq}=32$ rcode $=-2001$.

\section{Listing B.3: mrttest1.c}

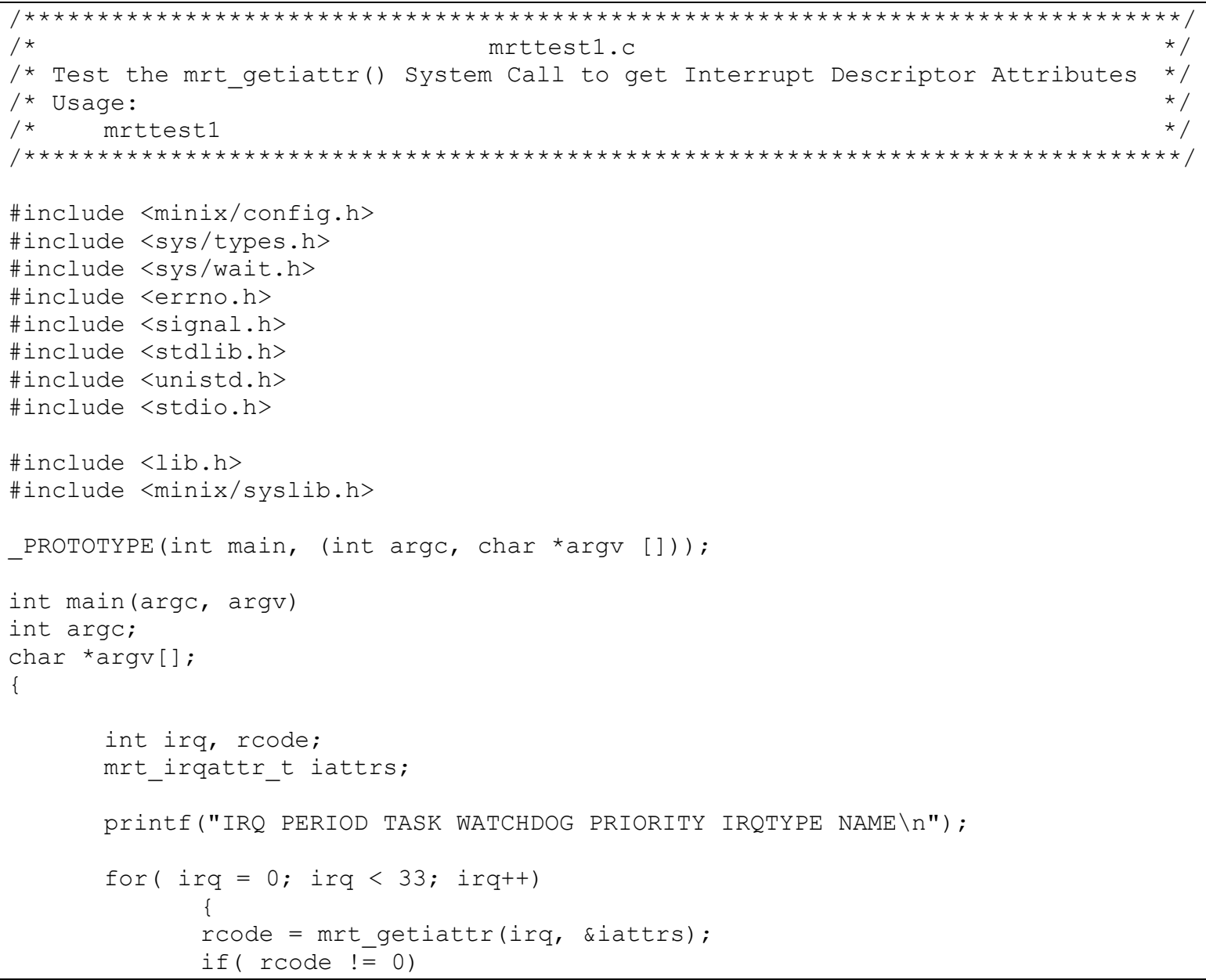




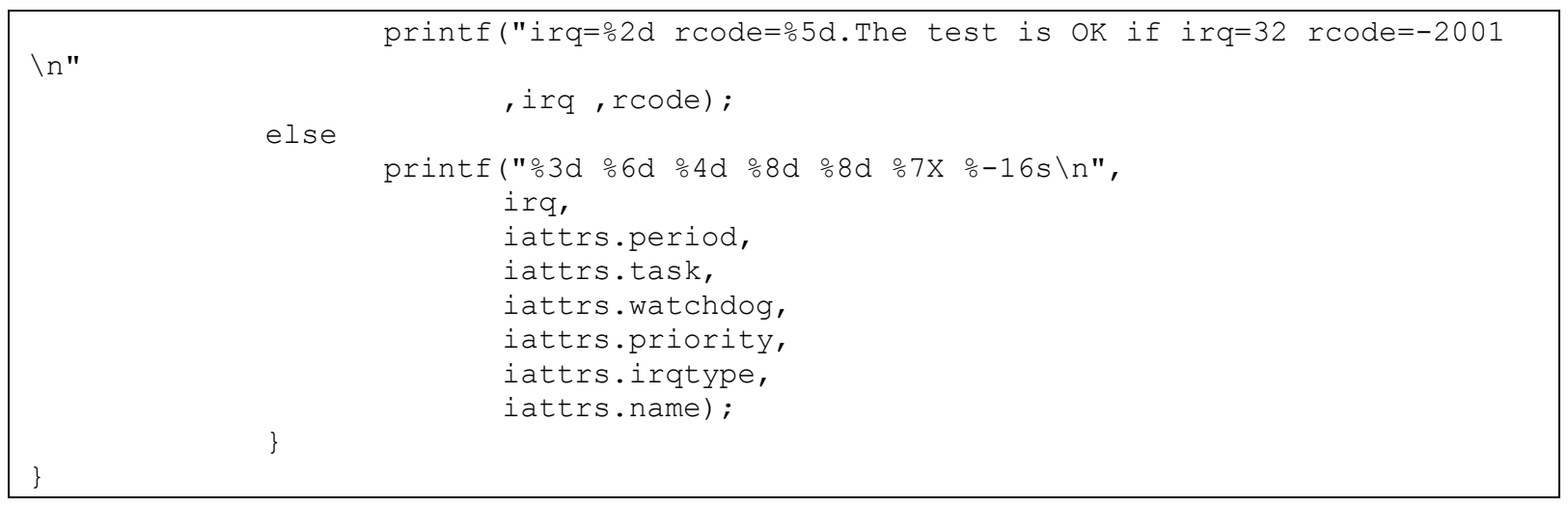

\section{B.4. mrttest1b.c}

This program gets and displays Interrrupt Descriptors of statistical fields. At the last line the test must display $i r q=32$ rcode $=-2001$.

\section{Listing B.4: mrttest1b.c}

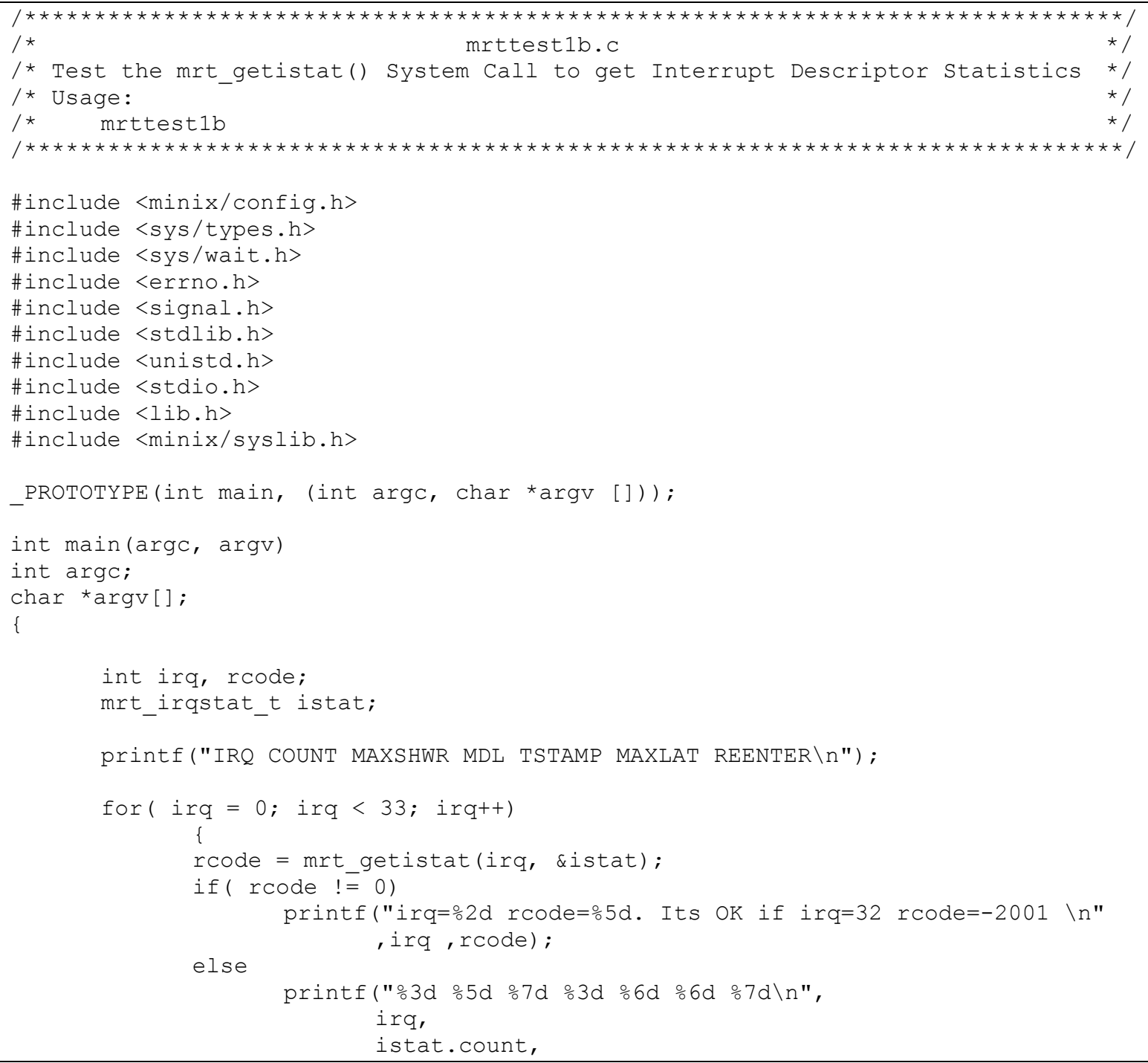




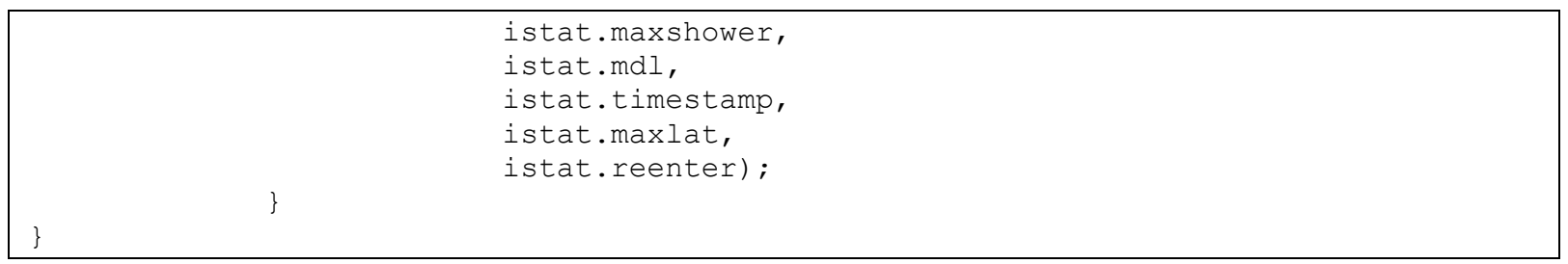

\section{B.5. mrttest1c.c}

This program gets and displays Interrrupt Descriptors of internal use fields. At the last line the test must display irq $=32$ rcode $=-2001$.

\section{Listing B.5: mrttest1c.c}

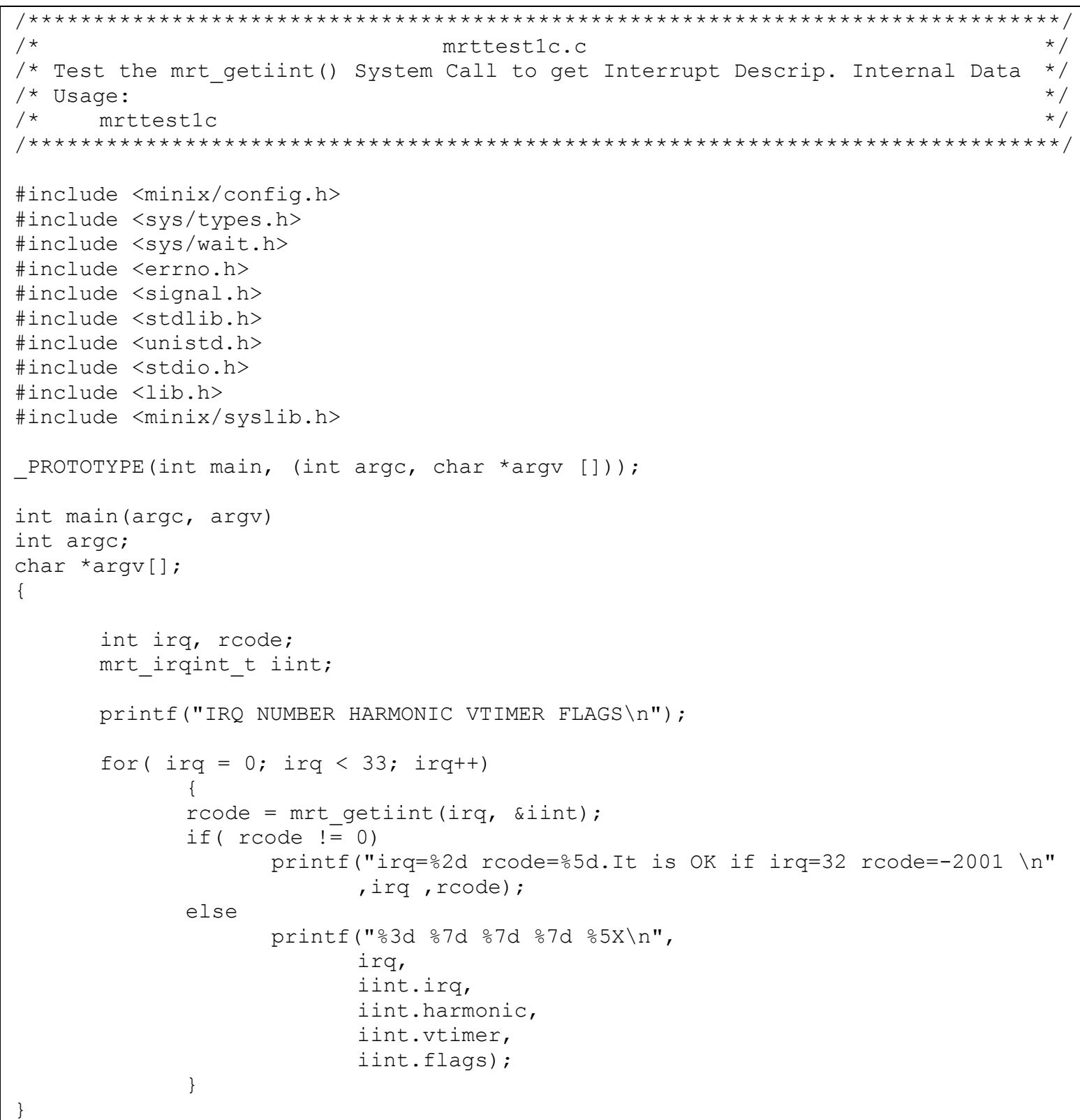




\section{B.6. mrttest2.c}

This program sets Interrrupt Descriptors processing attribute fields.

\section{Listing B.6: mrttest2.c}

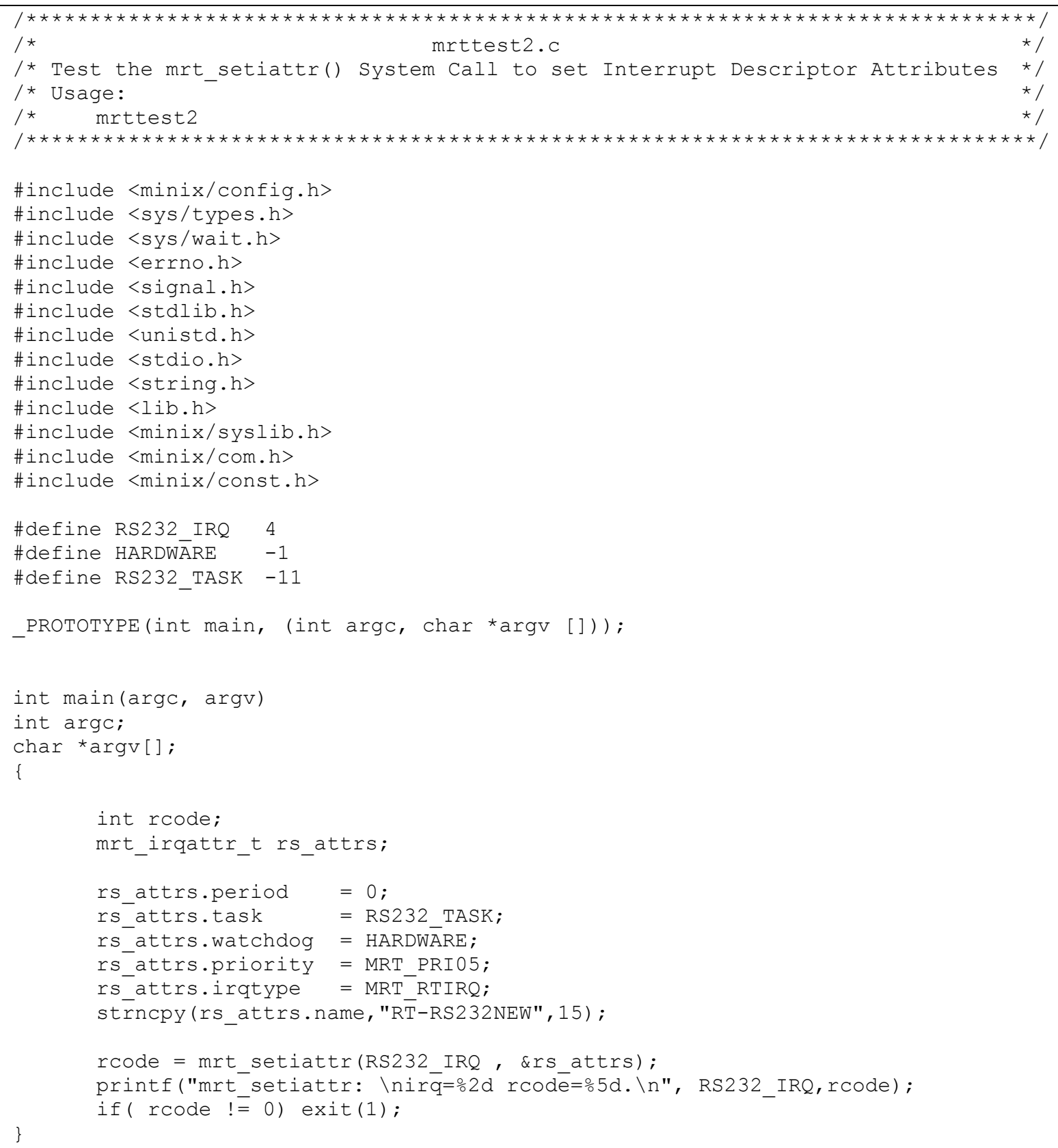

\section{B.7. mrttest3.c}

This program gets and displays System-wide statistics. 
Listing B.7: mrttest3.c

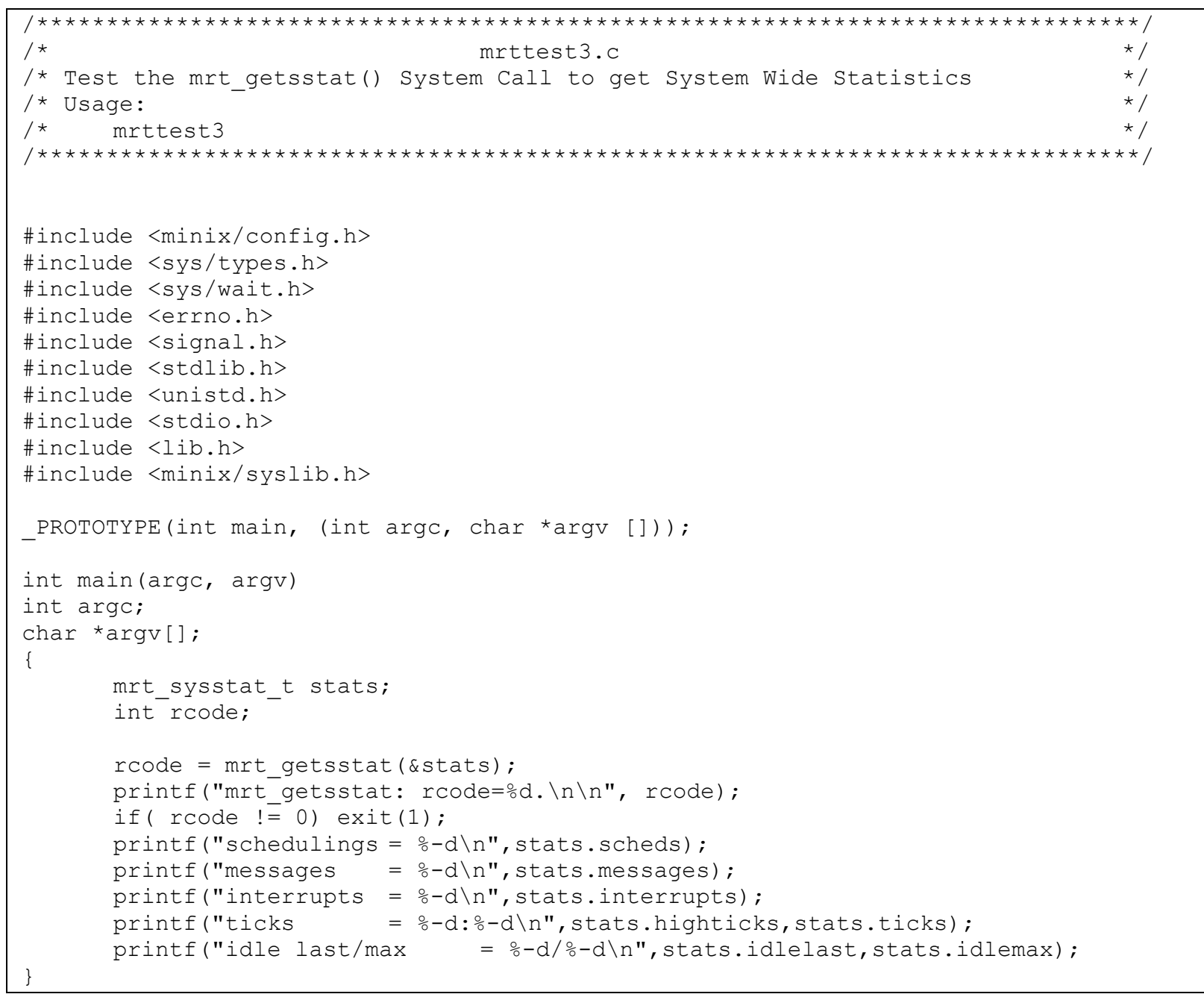

\section{B.8. mrttest3b.c}

This program displays a graph of CPU usage.

\section{Listing B.8: mrttest3b.c}

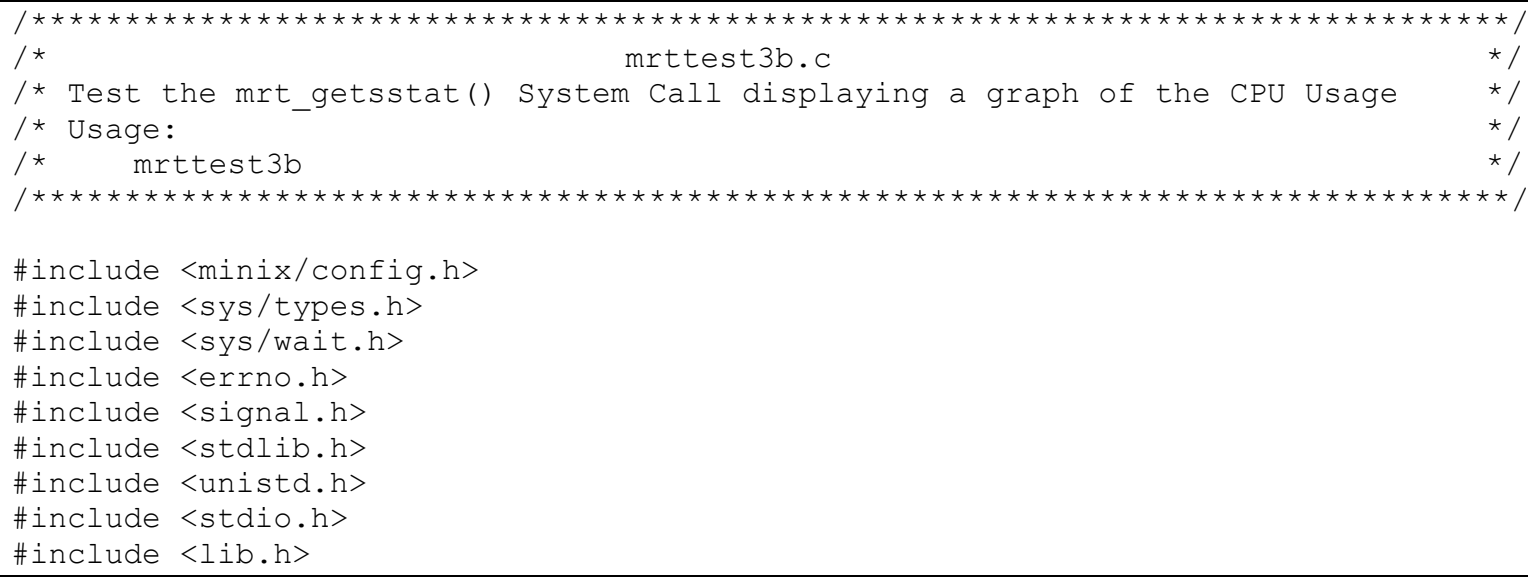




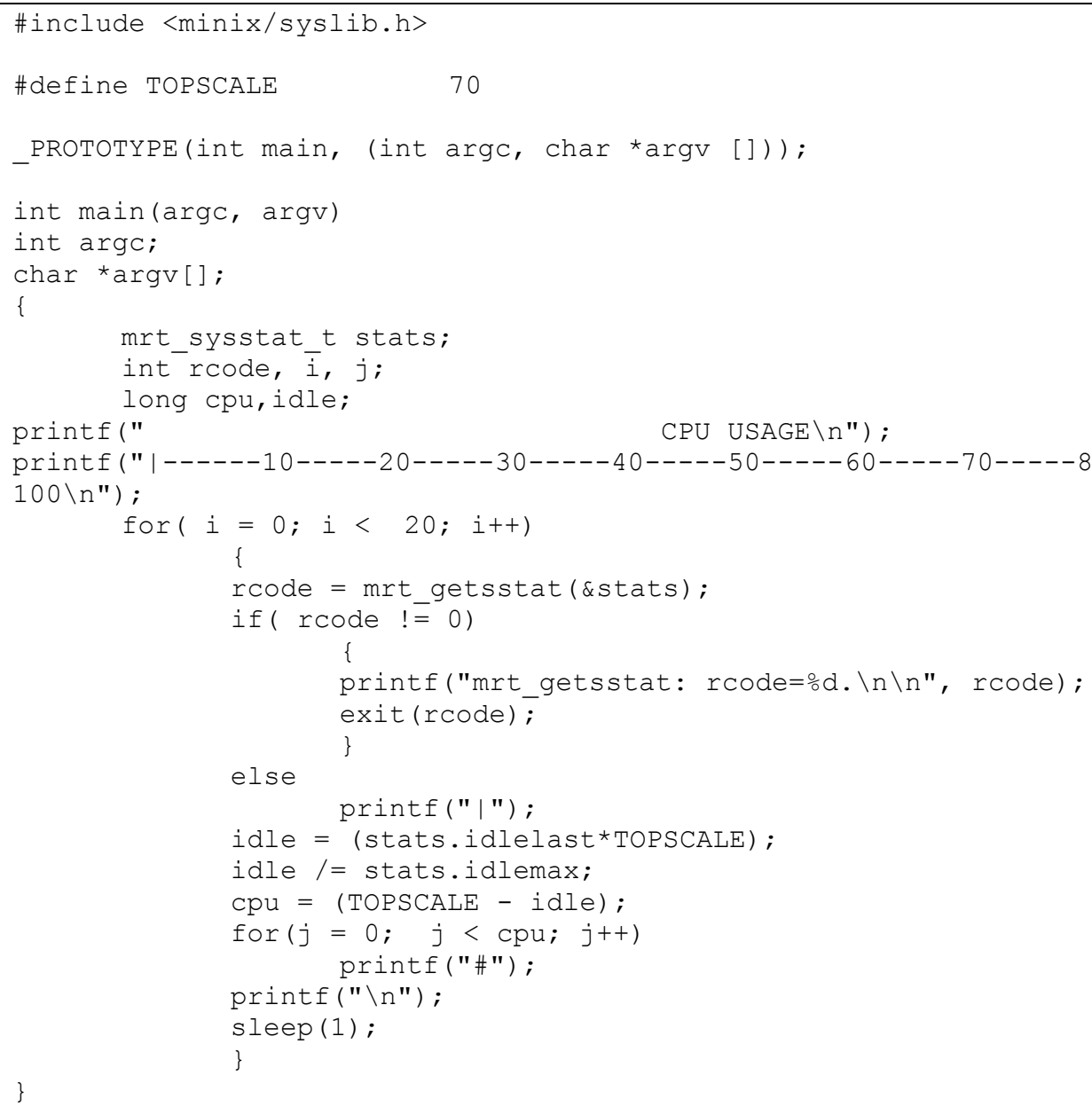

\section{B.9. mrttest3c.c}

This program gets and displays System processing values.

\section{Listing B.9: mrttest3c.c}

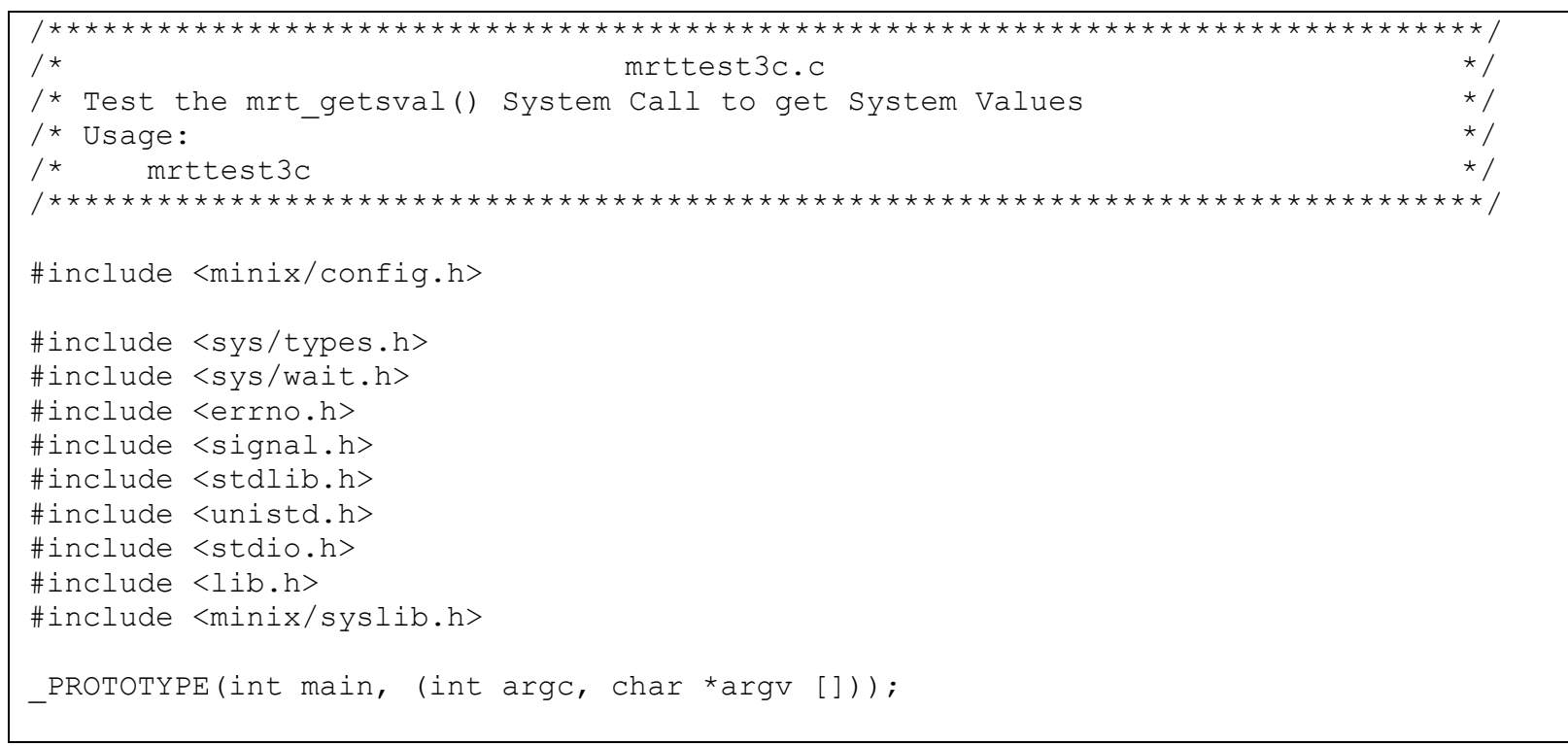




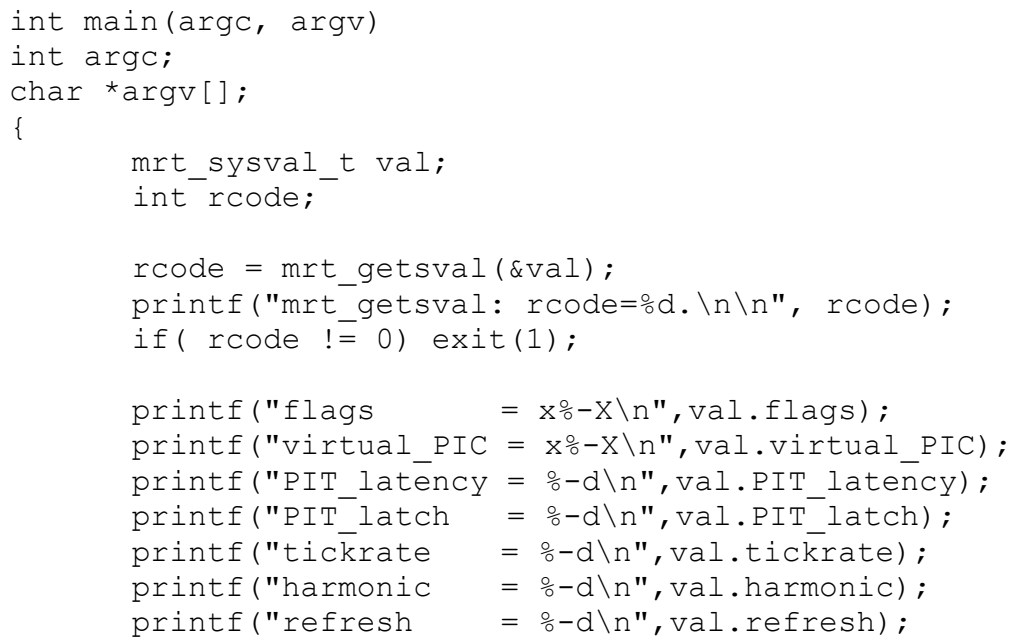

\section{B.10. mrttest4.c}

This program gets and displays Process Descriptor Attributes.

\section{Listing B.10: mrttest4.c}

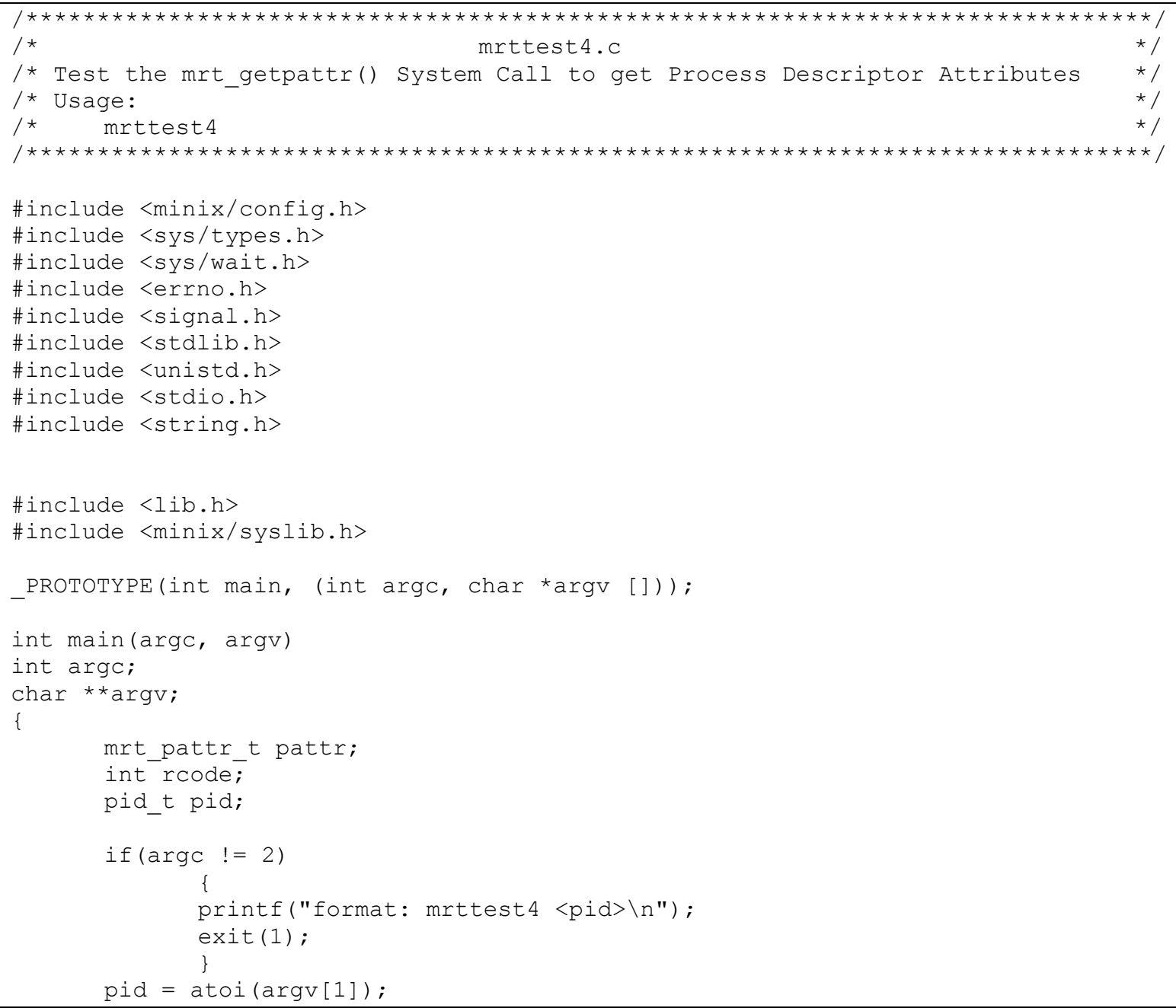




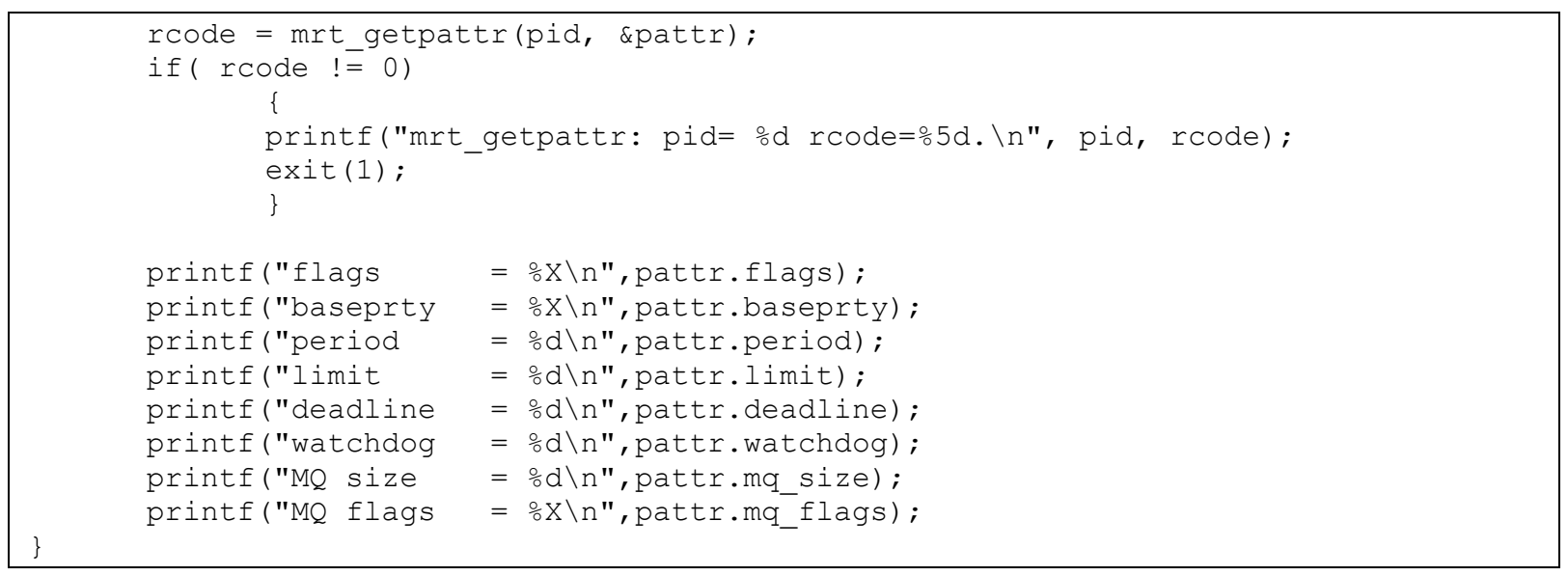

\section{B.11. mrttest $4 b . c$}

This program sets Process Descriptor Attributes.

\section{Listing B.11: mrttest4b.c}

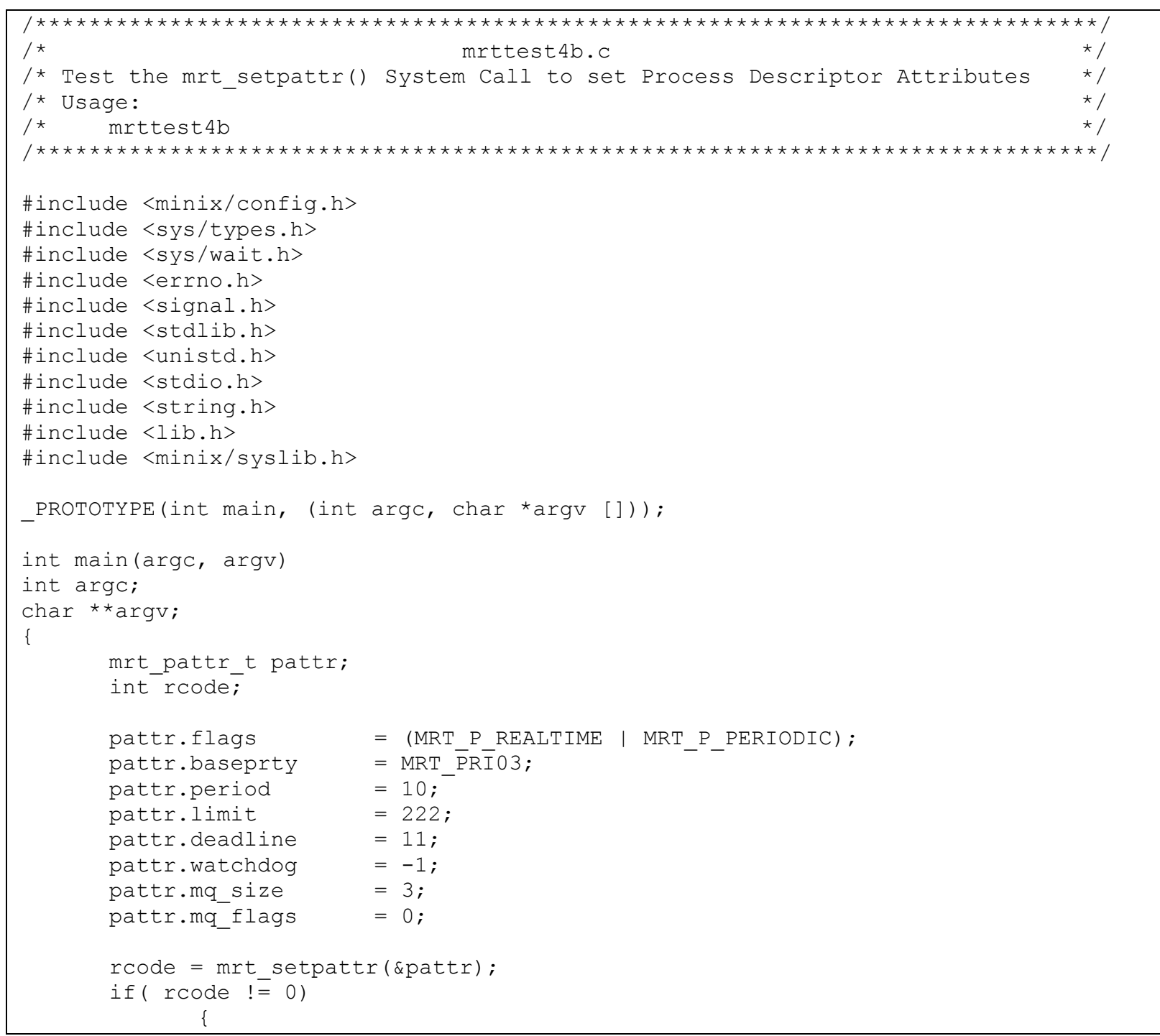




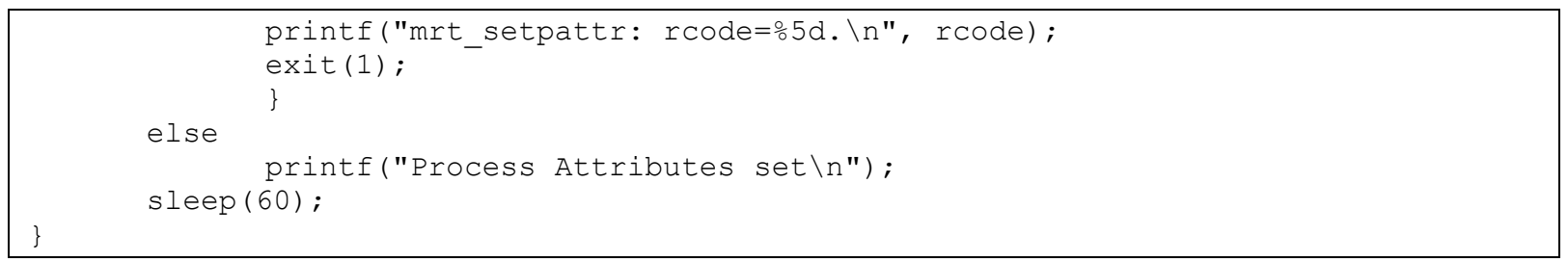

\section{B.12. mrttest5.c}

This program gets and displays Process Descriptor statistical fields.

\section{Listing B.12: mrttest5.c}

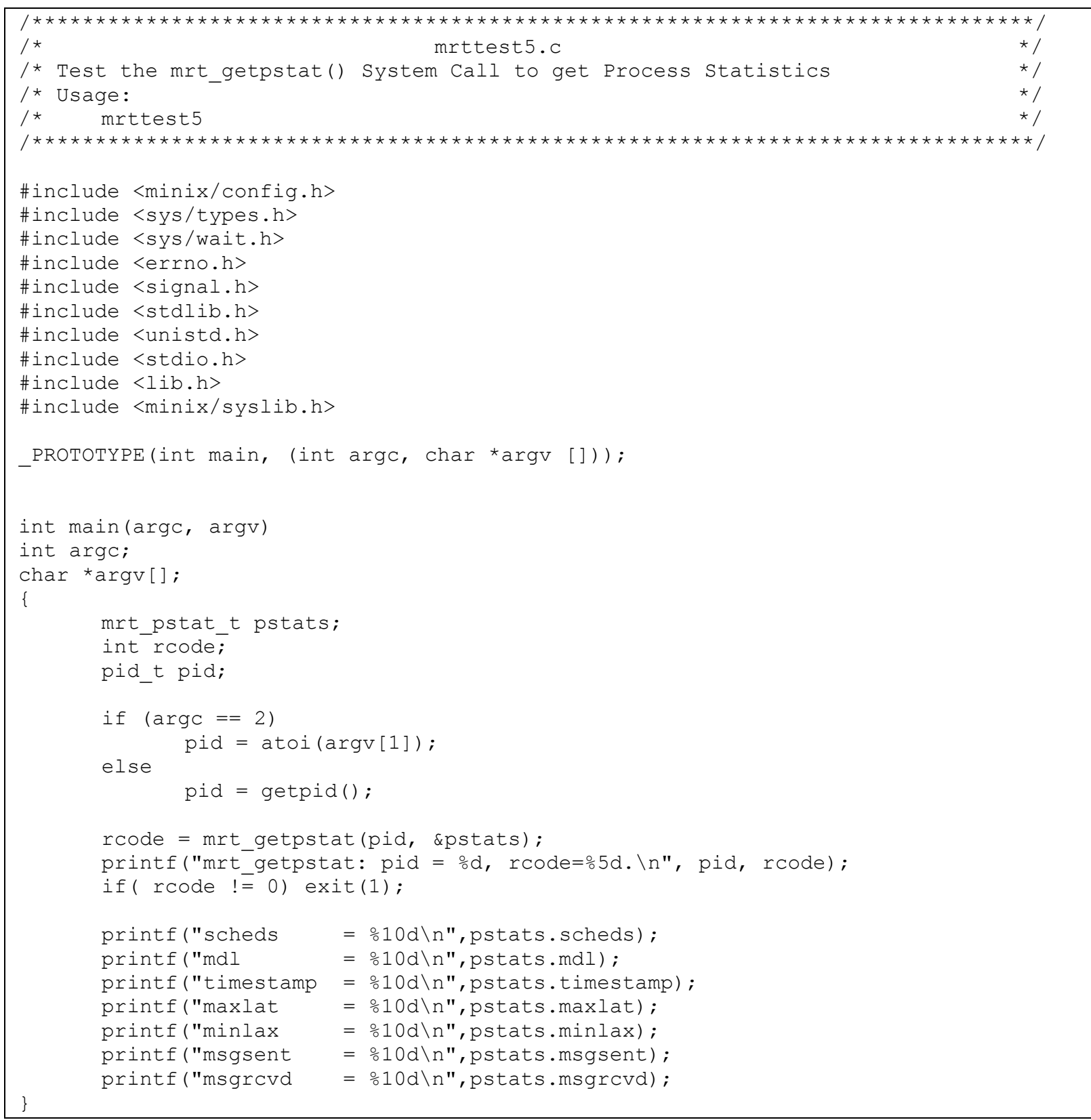




\section{B.13. mrttest5b.c}

This program gets and displays Process Descriptor internal use fields.

\section{Listing B.13: mrttest5b.c}

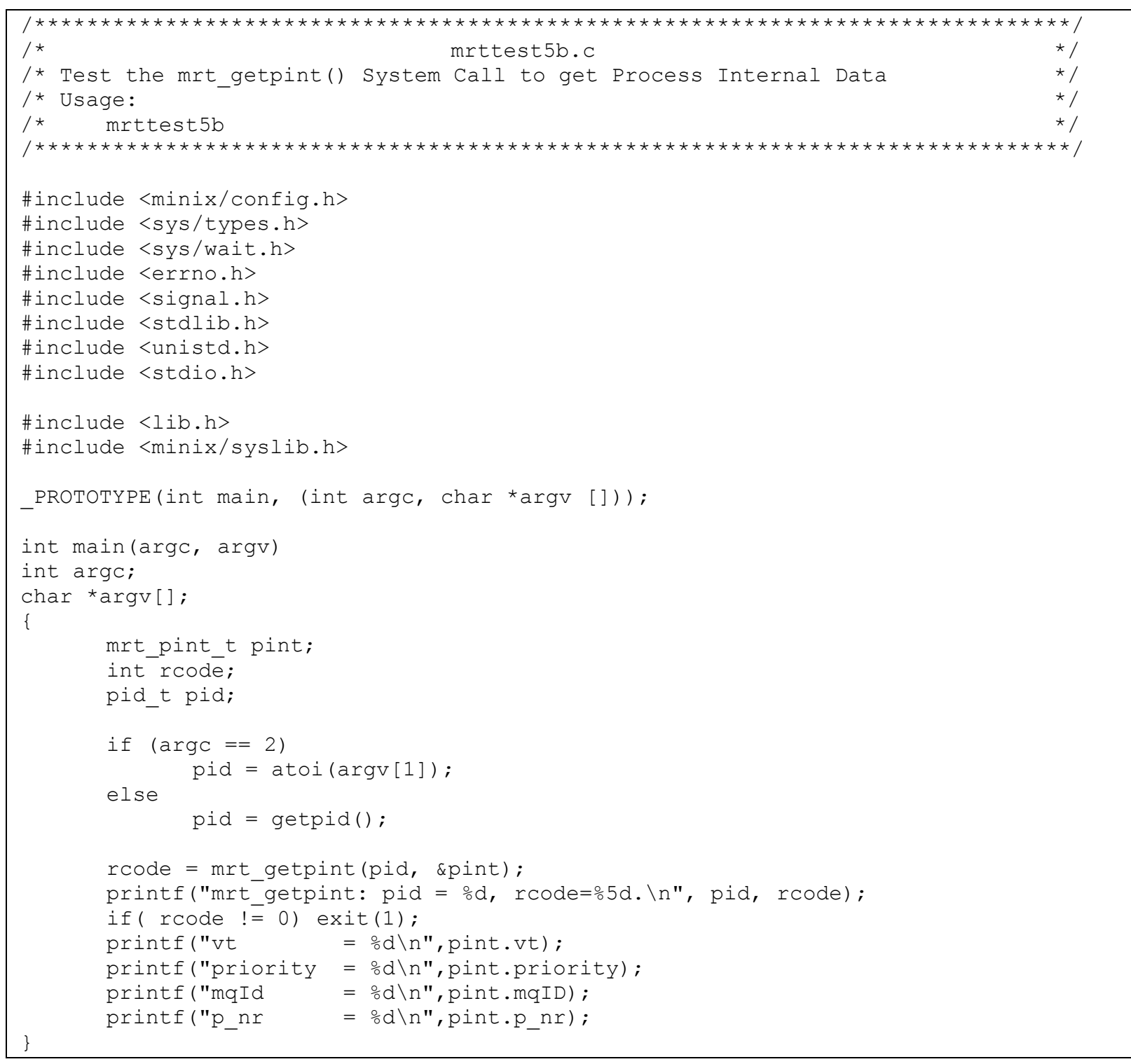

\section{B.14. mrttest5c.c}

This program clears Process Descriptor statistical fields.

Listing B.14: mrttest5c.c

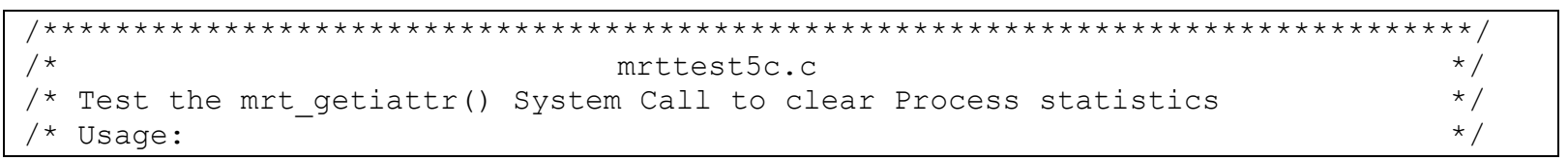




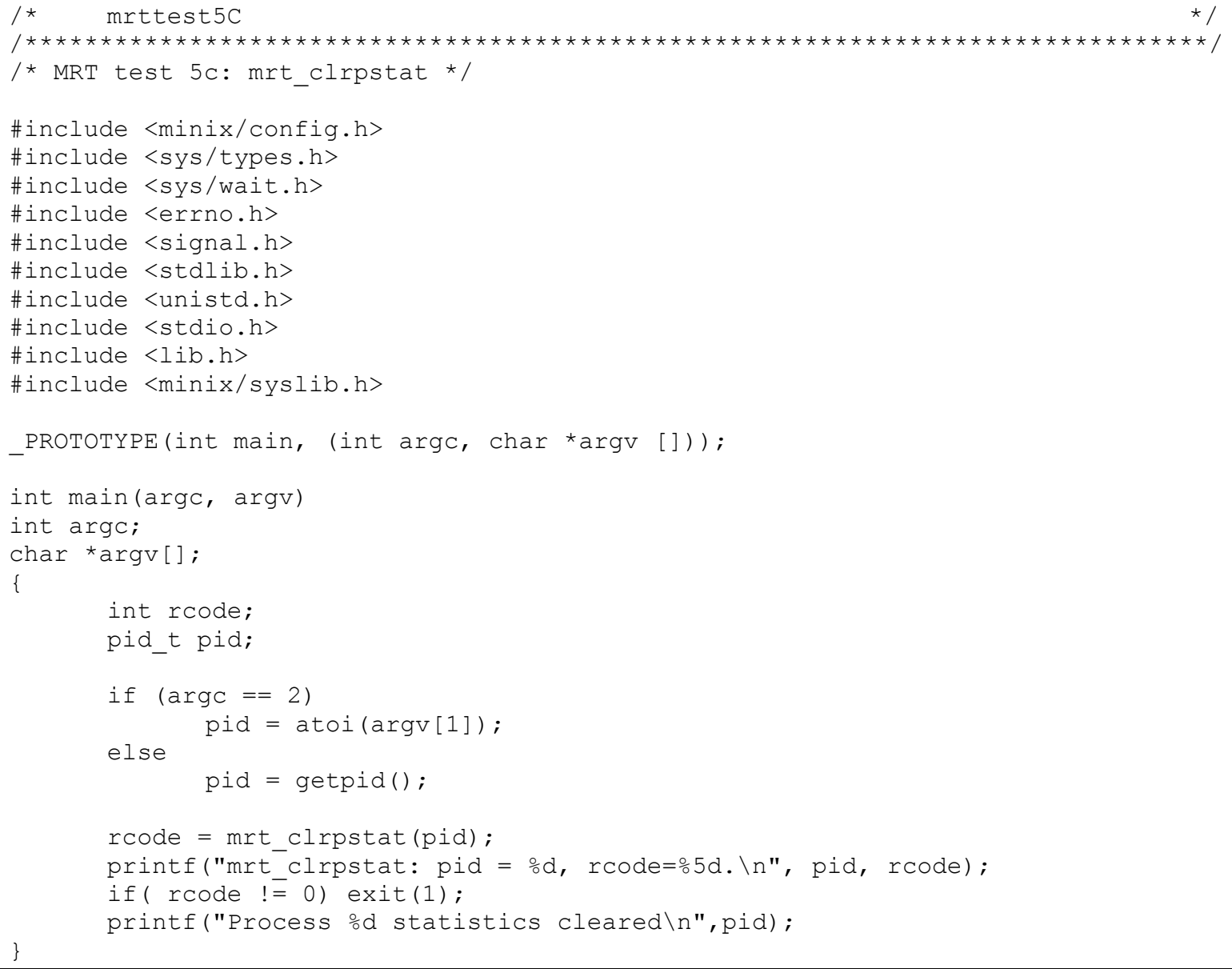

\section{B.15. mrttest6.c}

This program:

- Sets its process descriptor attributes to convert itself into a RT-process.

- Converts itself the process is converted into a RT-process.

- Converts itself back into a NRT-process.

- Displays its process descriptor statistics.

\section{Listing B.15: mrttest6.c}

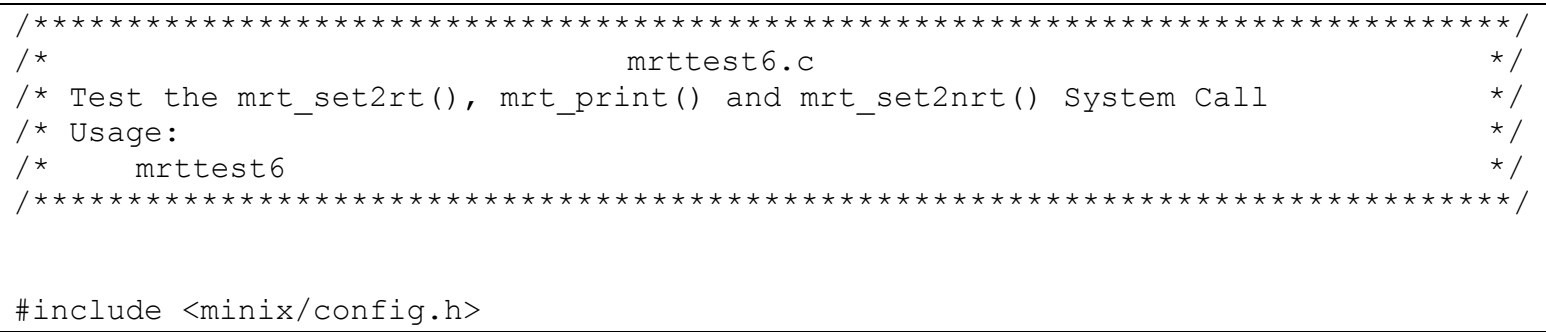




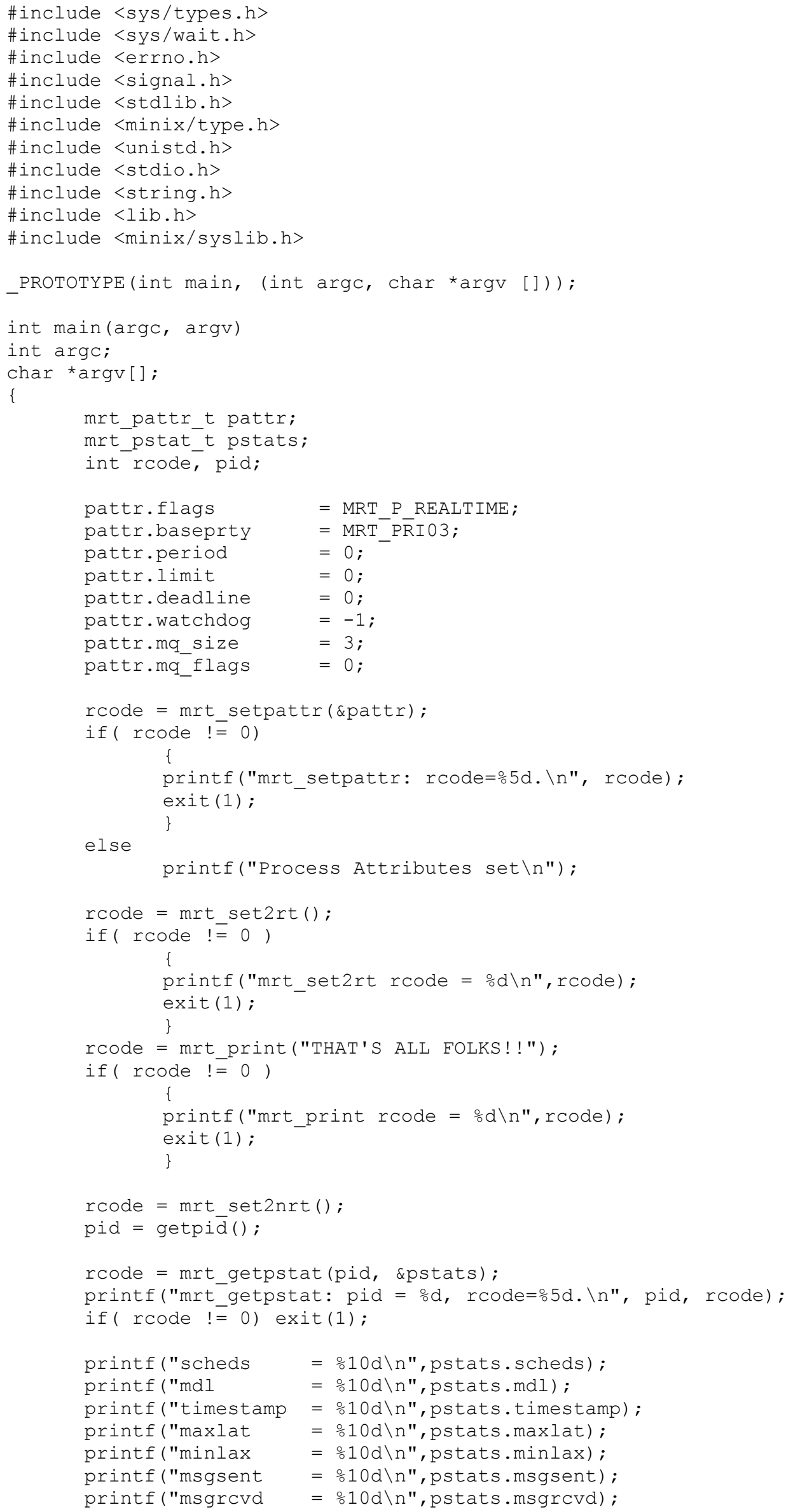




\section{B.16. mrttest6b.c}

This program:

- Sets its process descriptor attributes to convert itself into a RT-process.

- Converts itself the process is converted into a RT-process.

- Prints a text on the system console using mrt_print() Kernel Call.

- Exits without calling mrt_set2nrt()Kernel Call.

\section{Listing B.16: mrttest6b.c}

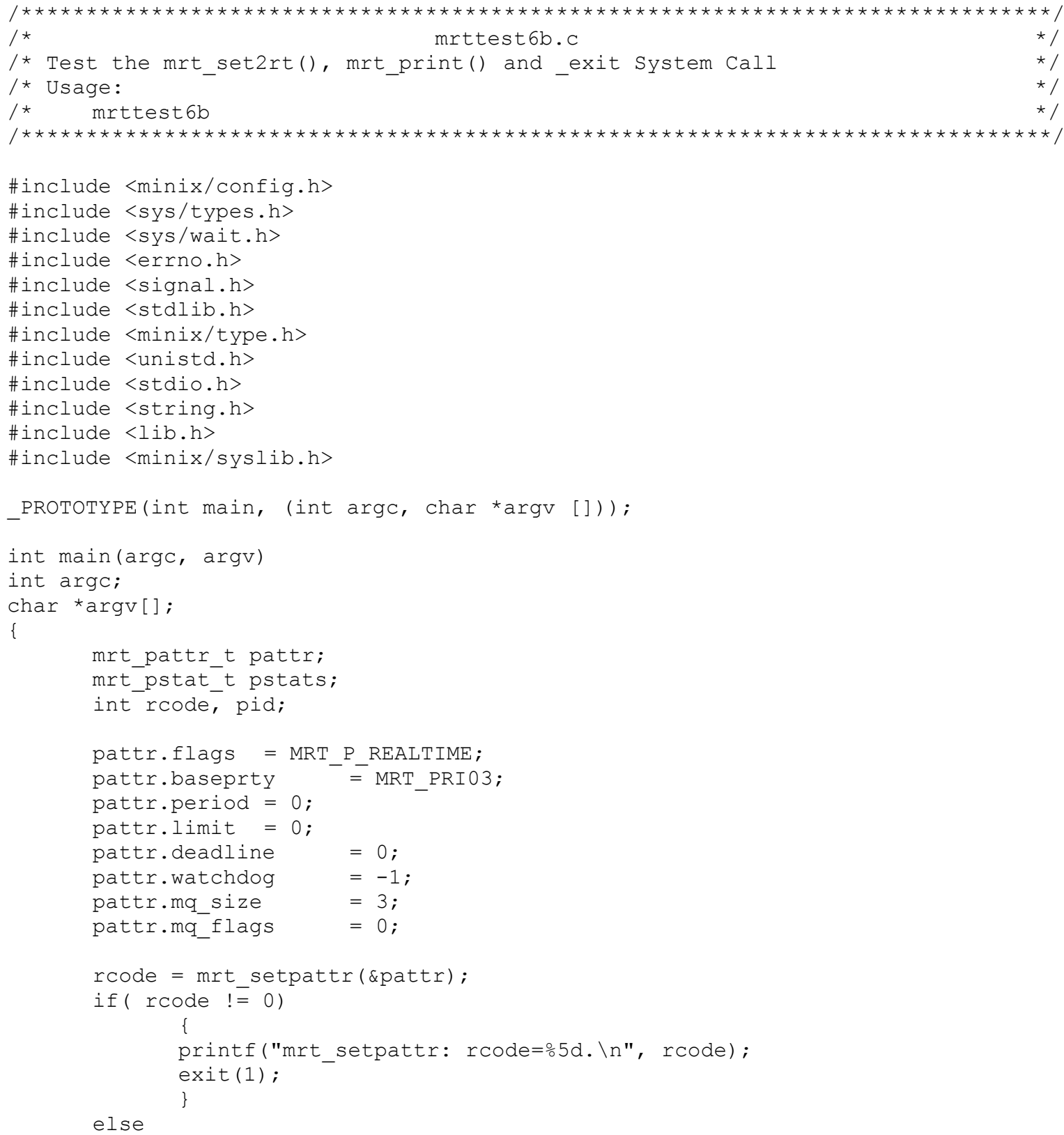




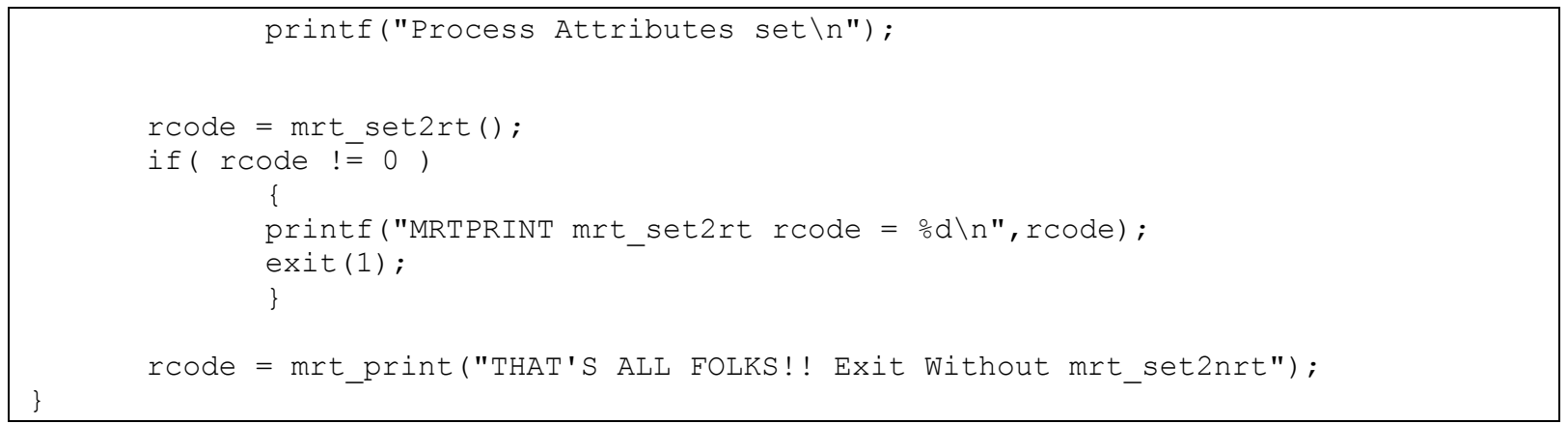

\section{B.17. mrttest6c.c}

This program:

- Sets its process descriptor attributes to convert itself into a RT-process.

- Converts itself into a RT-process.

- $\quad$ Prints a text on the system console using mrt_print() Kernel Call.

- Blocks itself by 2000 Timer ticks

- Converts itself back into a NRT-process.

\section{Listing B.17: mrttest6c.c}

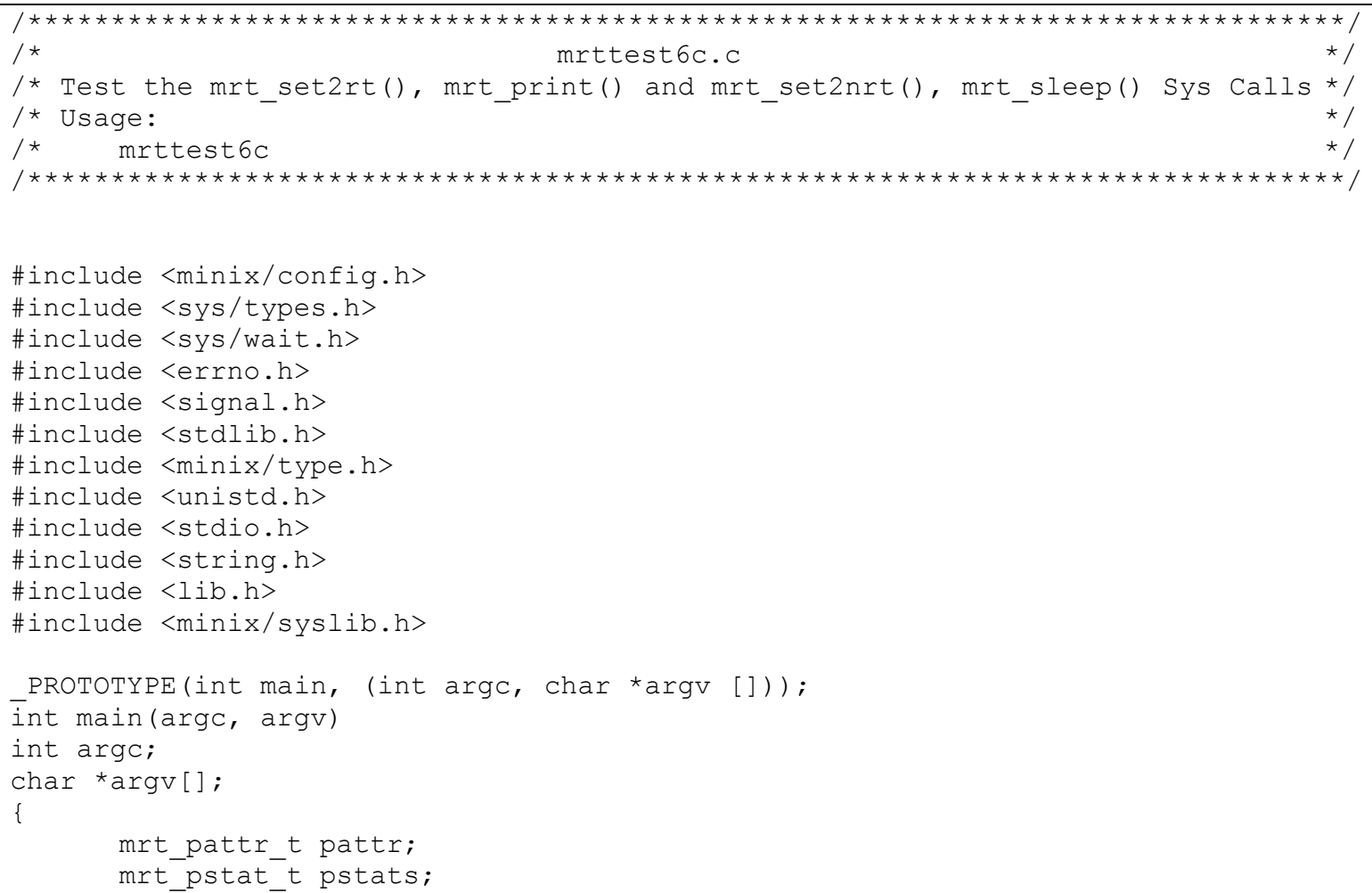




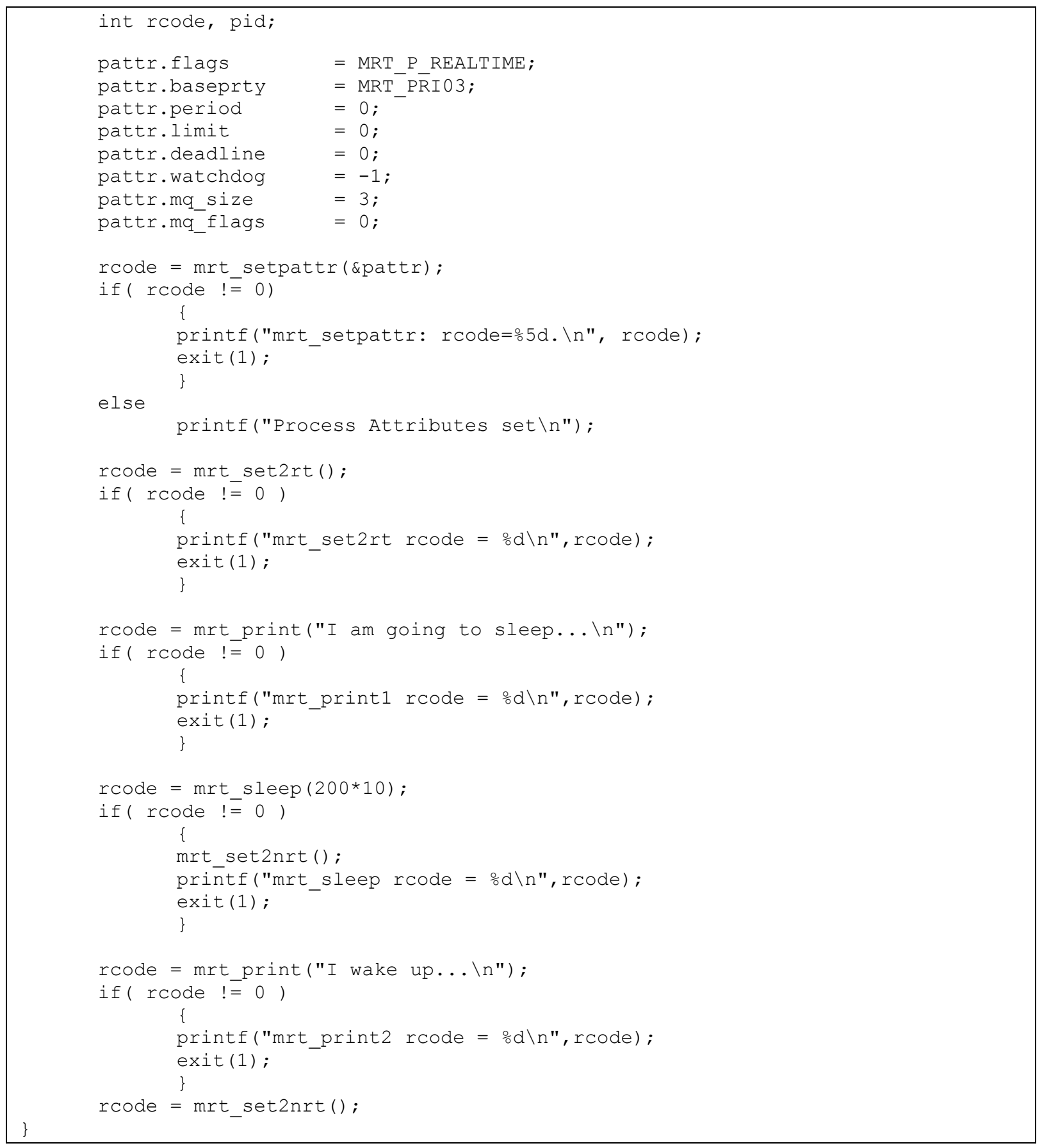

\section{B.18. mrttest6d.c}

This program forks into two processes, the FATHER and the SON:

The FATHER:

- Gets SON's process descriptor internal values to obtain its RT-PID.

- Sets its process descriptor attributes to convert itself into a RT-process. 
- Converts itself into a RT-process.

- Prints a text on the system console using mrt_print() Kernel Call.

- Wakes up SON using mrt_wakeup() Kernel Call.

- Converts itself back into a NRT-process.

The SON:

- Sets its process descriptor attributes to convert itself into a RT-process.

- Converts itself into a RT-process.

- Prints a text on the system console using mrt_print() Kernel Call.

- Sleeps (using mrt_sleep() Kernel Call) until FATHER will wake up it

- Converts itself back into a NRT-process.

\section{Listing B.18: mrttest6d.c}

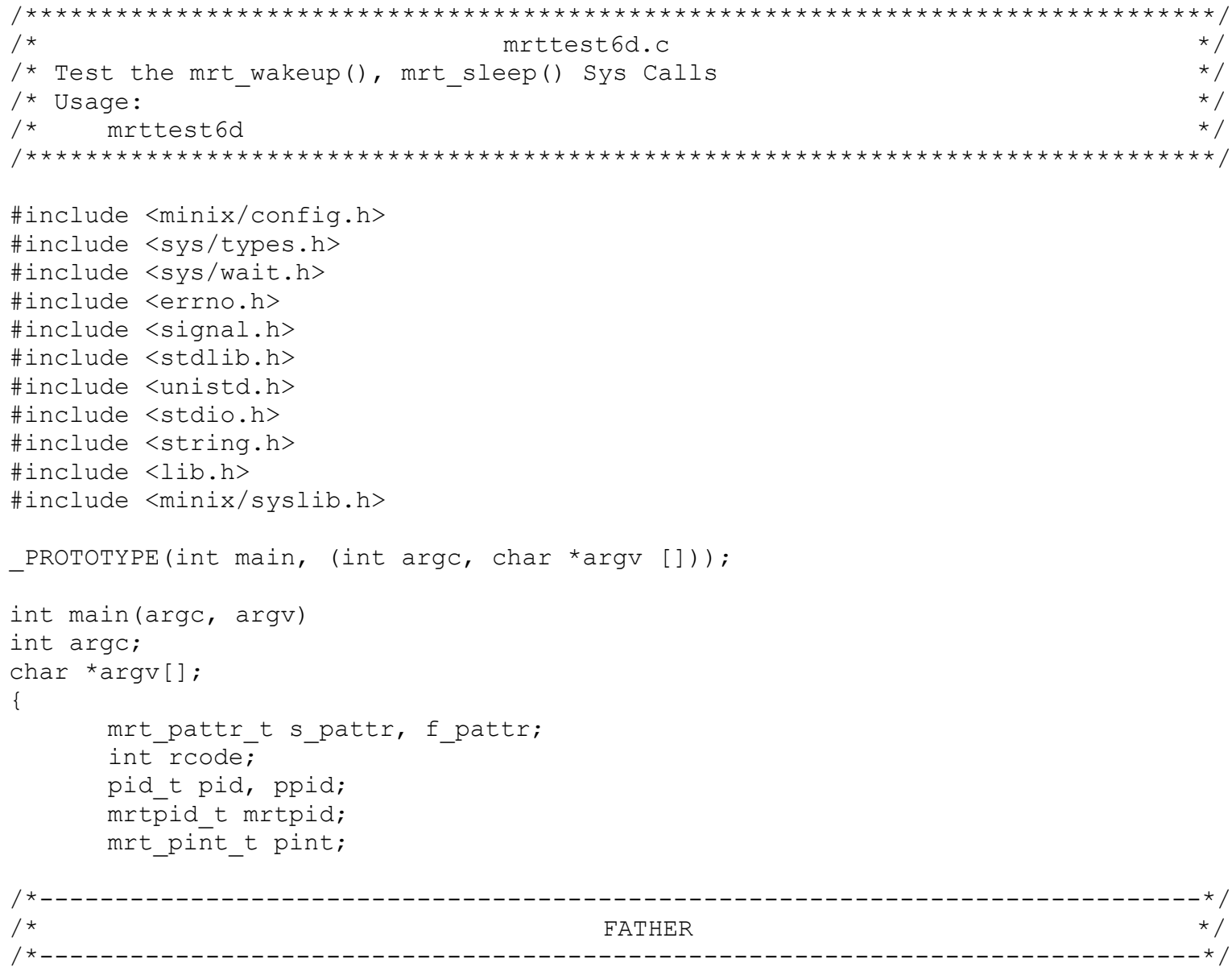




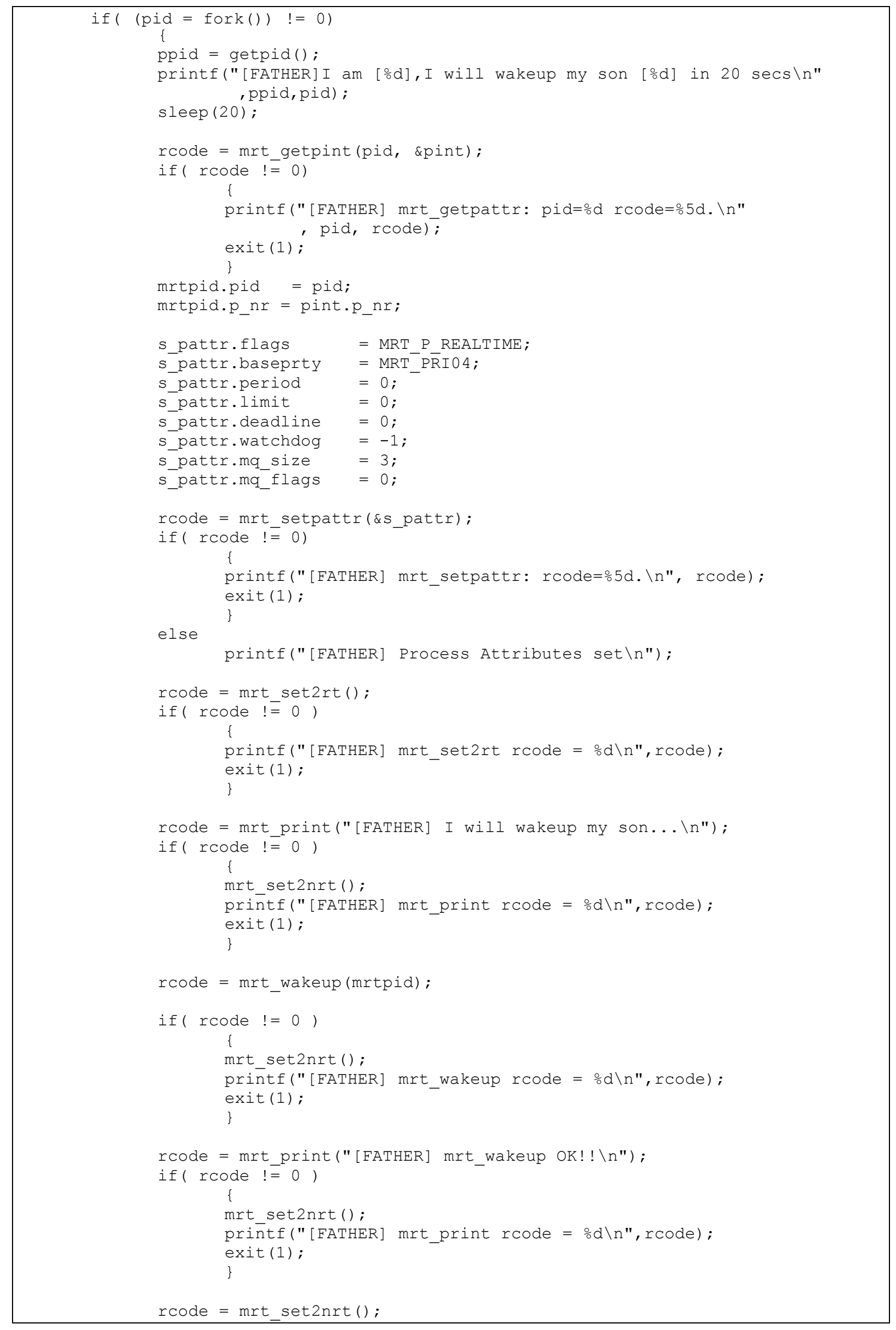




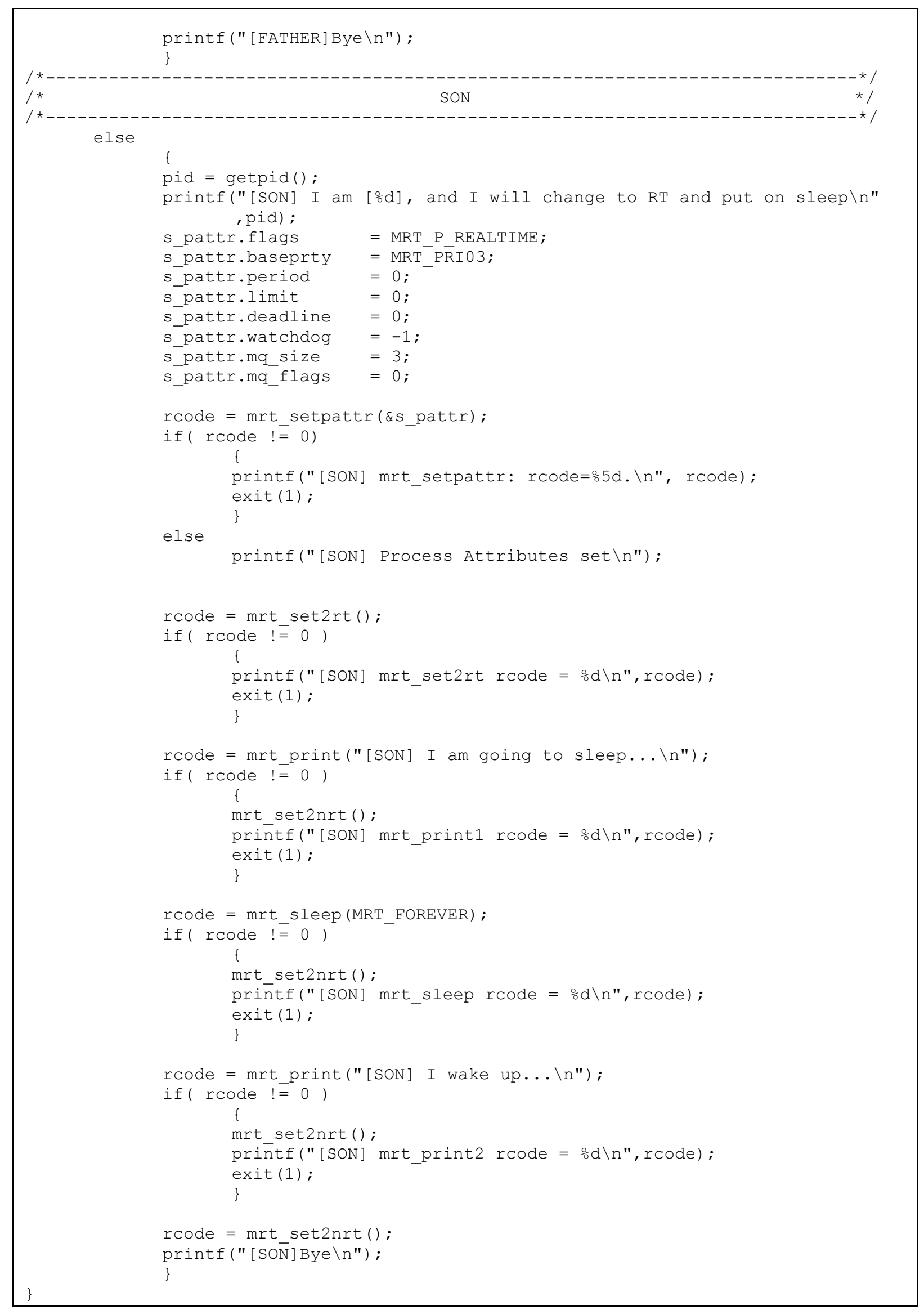




\section{B.19. mrttest6e.c}

This program forks into eleven processes, the FATHER and ten SONs:

The FATHER:

- Gets SONs' process descriptor internal values to obtain their RT-PID.

- Sets its process descriptor attributes to convert itself into a RT-process.

- Converts itself into a RT-process.

- Prints a text on the system console using mrt_print() Kernel Call.

- Wakes up ten SONs using mrt_wakeup() Kernel Call.

- Converts itself back into a NRT-process.

The SONs:

- Set their process descriptor attributes to convert themselves into RTprocesses.

- Convert themselves into RT-processes.

- Print texts on the system console using mrt_print() Kernel Calls.

- Sleep (using mrt_sleep() Kernel Call) until FATHER will wake up them.

- Convert themselves back into NRT-processes.

Listing B.19: mrttest6e.c

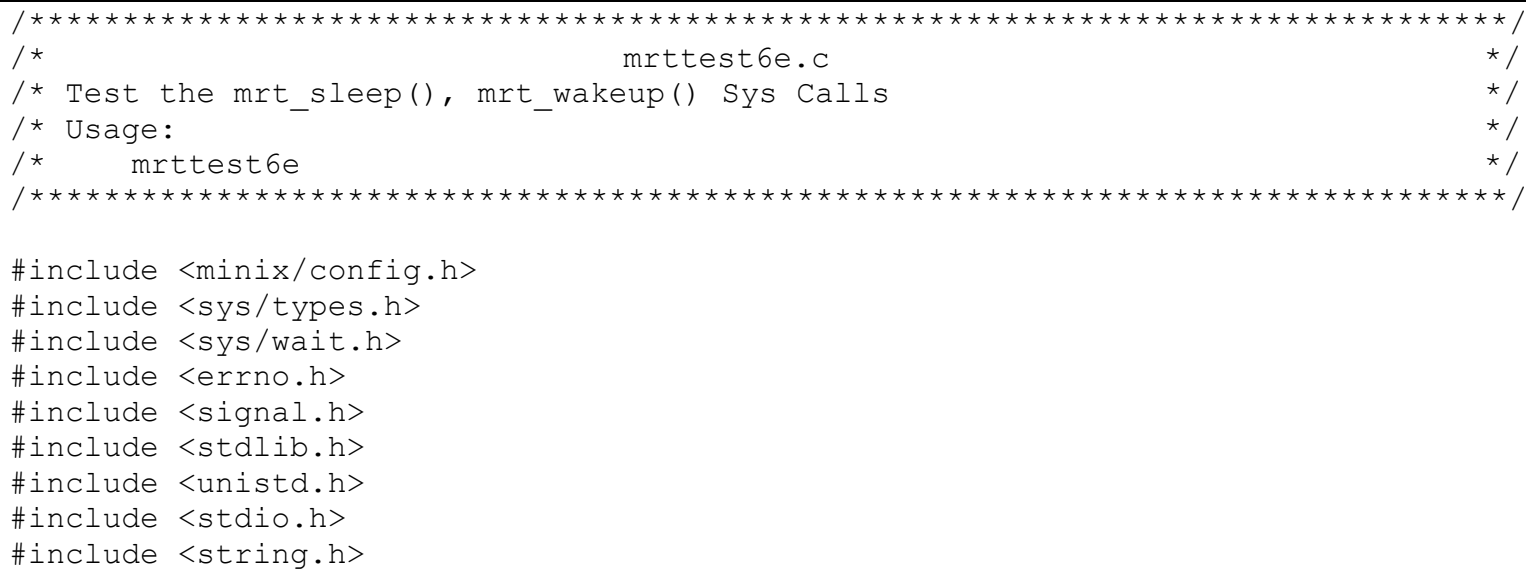




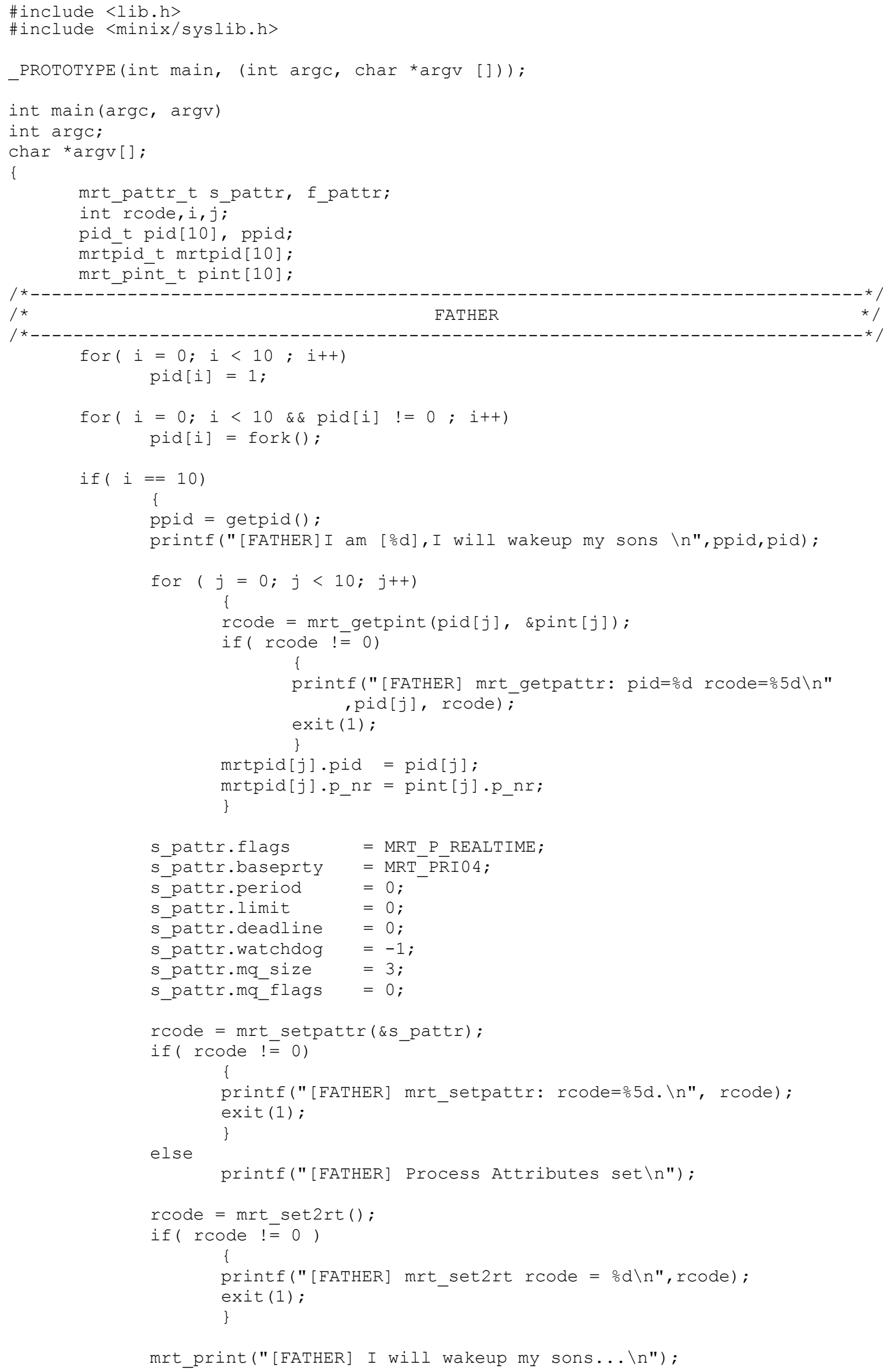




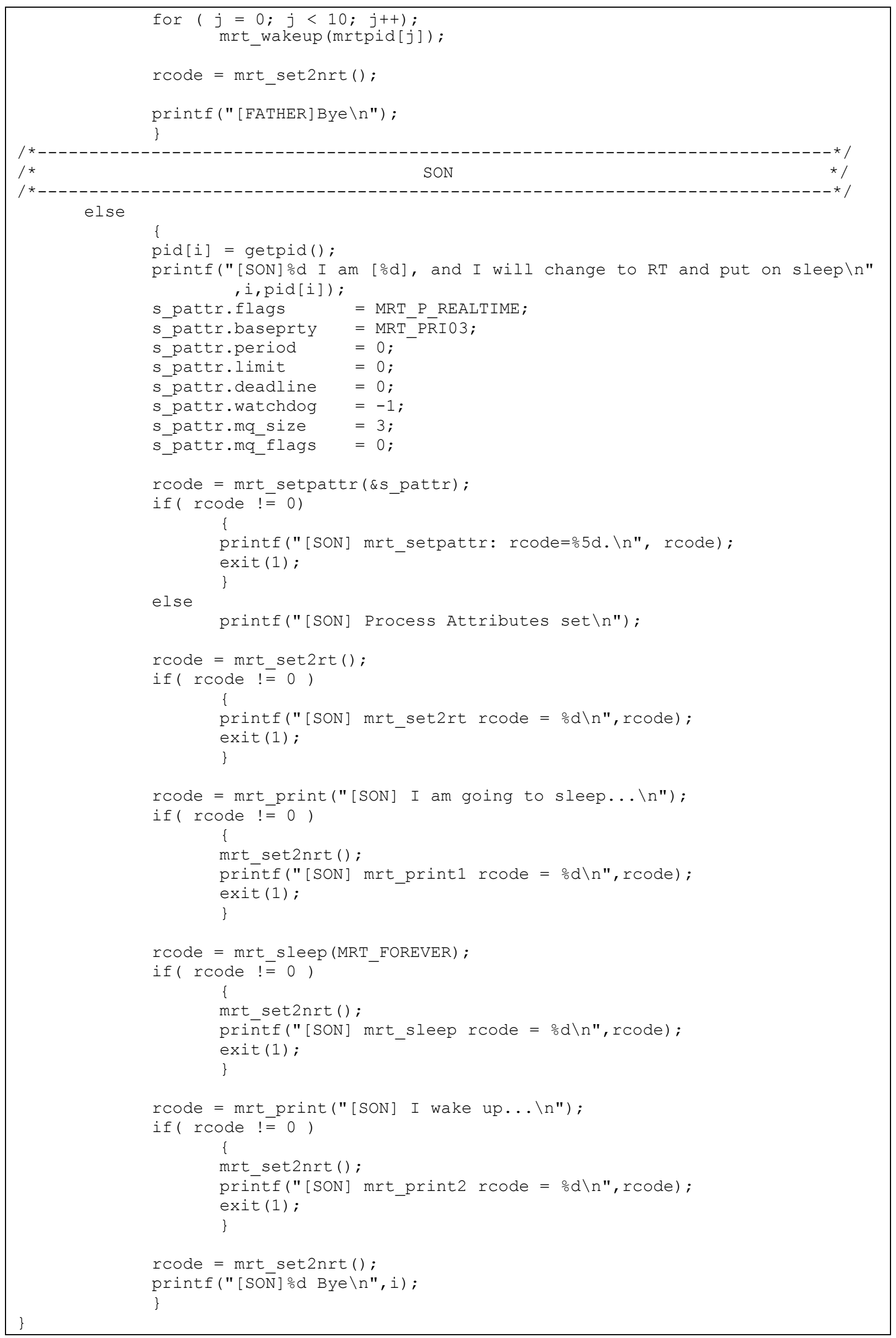




\section{B.20. mrttest7.c}

This program test periodic processing.

- Sets its process descriptor attributes to convert itself into a Periodic RTprocess.

- Converts itself into a RT-process.

- Loops until it processing limit is reached

- Prints a text on each loop.

- When the processing limit is reached the process is set in stopped setting the MRT_STOP bit of its flags.

\section{Listing B.20: mrttest7.c}

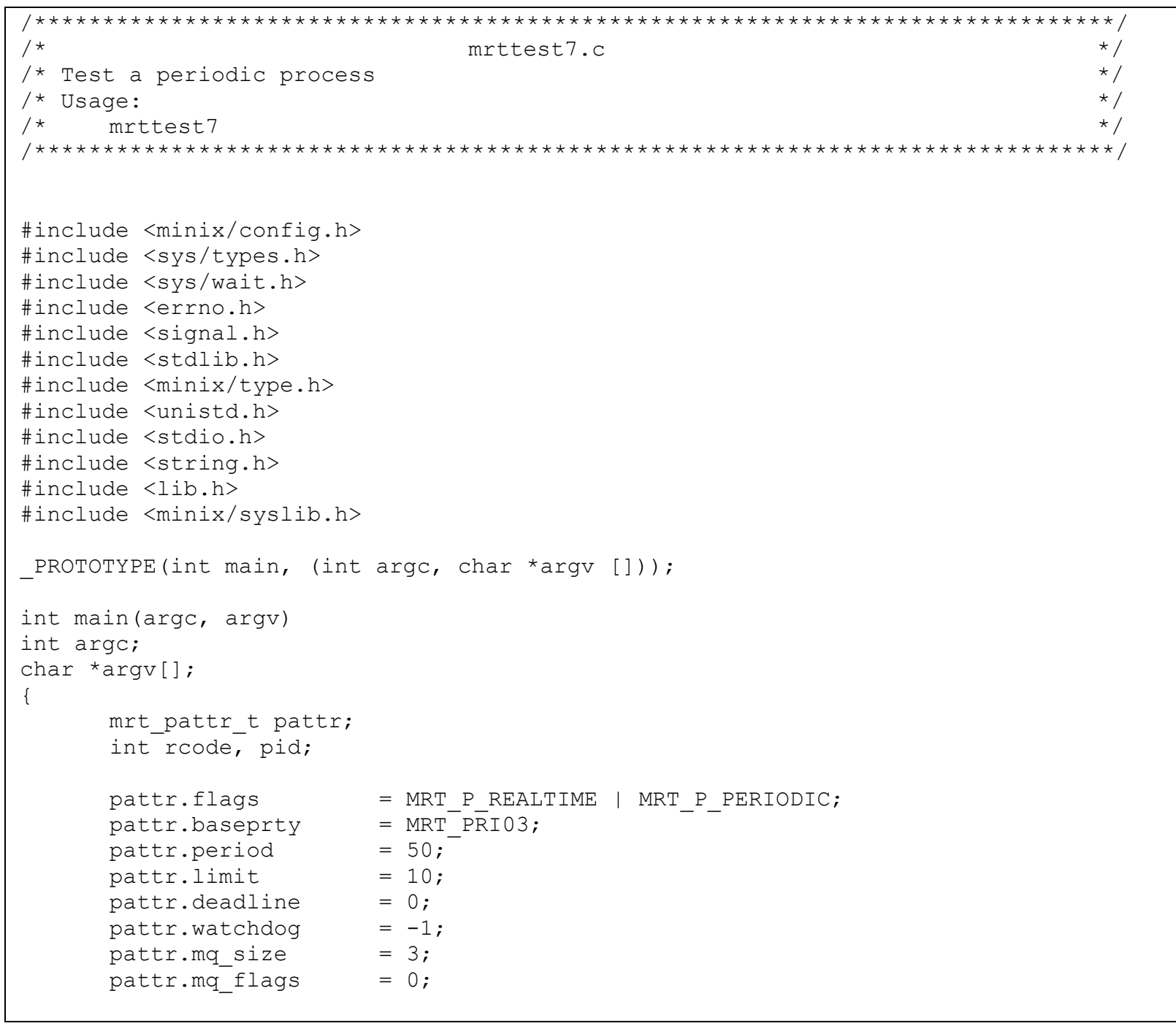




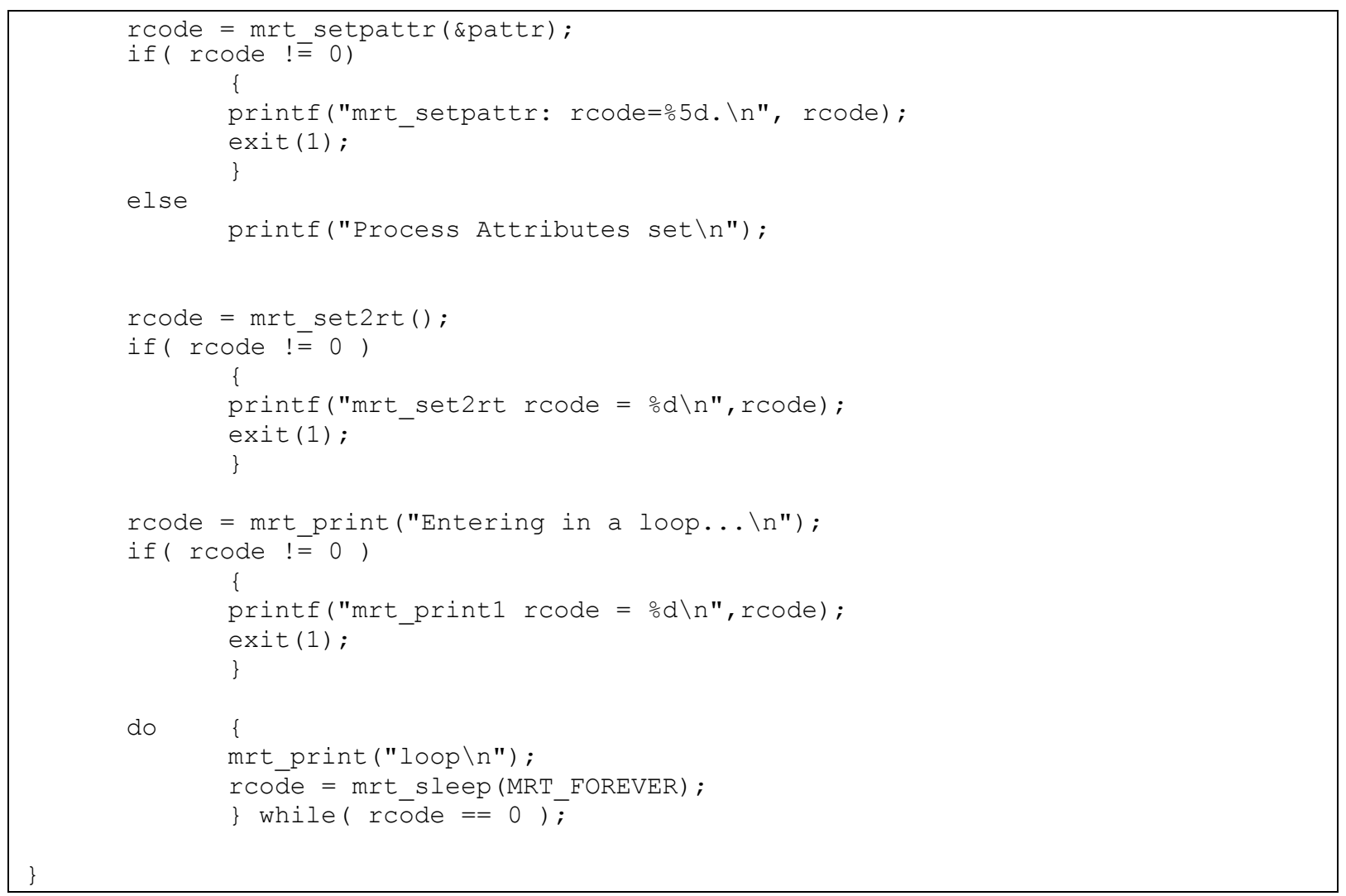

\section{B.21. mrttest7b.c}

This program test periodic processing.

- Sets its process descriptor attributes to convert itself into a Periodic RTprocess.

- Converts itself into a RT-process.

- Loops five times.

- Prints a text on each loop.

- Converts itself back into a NRT-process.

Listing B.21: mrttest7b.c

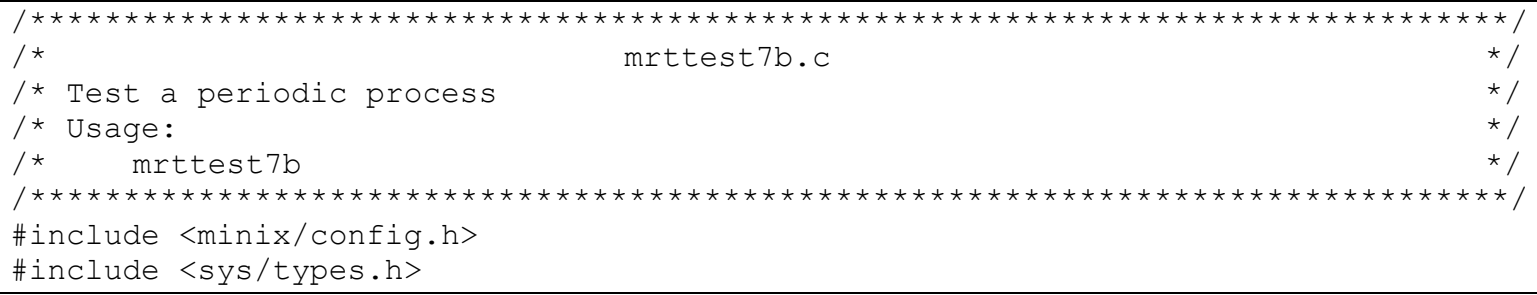




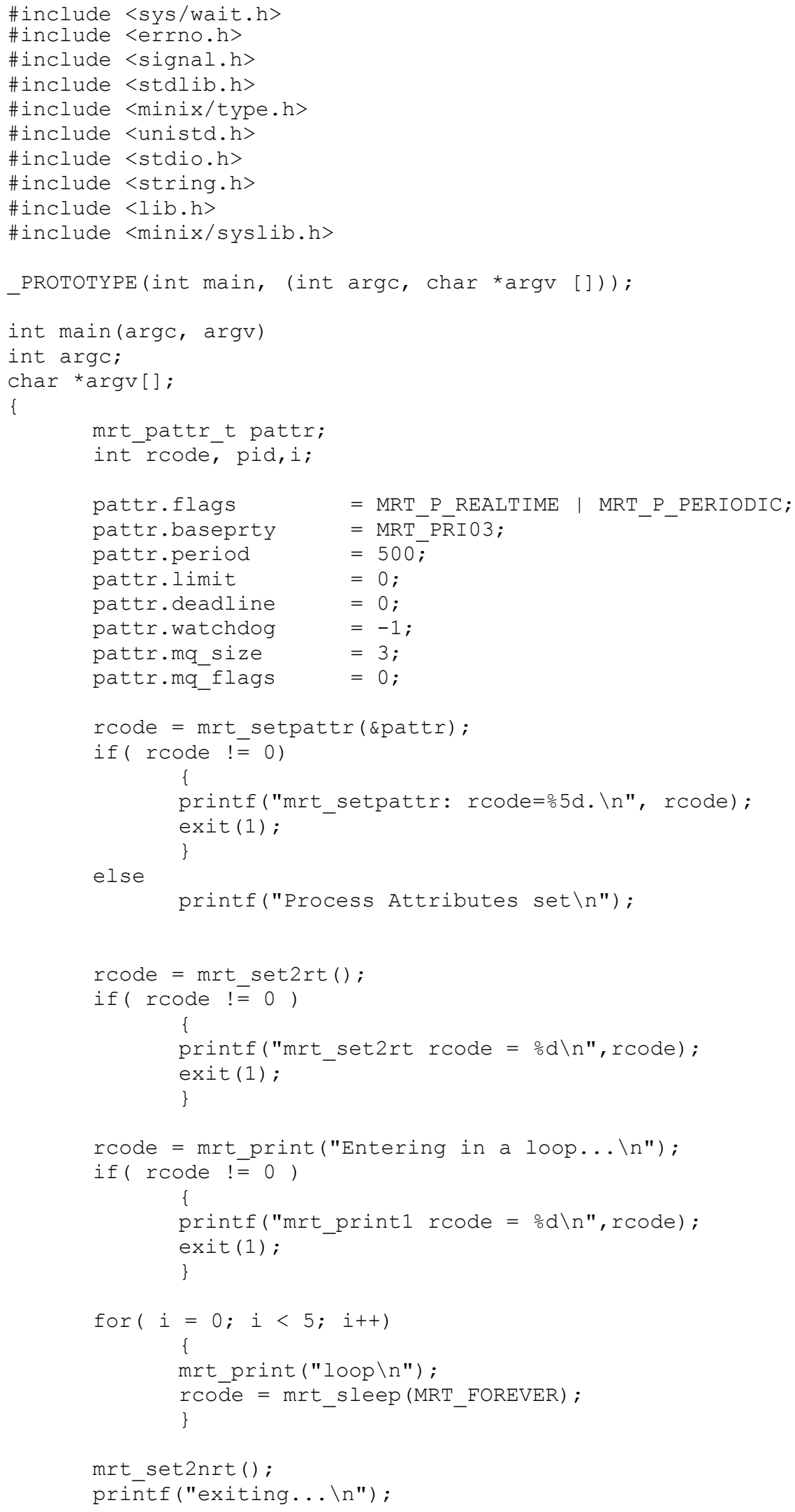




\section{B.22. mrttest8.c}

This program forks into two processes, the FATHER and the SON:

The FATHER:

- Gets SON's process descriptor internal values to obtain its RT-PID.

- Sets its process descriptor attributes to convert itself into a RT-process.

- Converts itself into a RT-process.

- Prints a text on the system console using mrt_print() Kernel Call.

- Send a request message to SON using mrt_rqst() Kernel Call.

- Converts itself back into a NRT-process.

The SON:

- Sets its process descriptor attributes to convert itself into a RT-process.

- Converts itself into a RT-process.

- Prints a text on the system console using mrt_print() Kernel Call.

- Sleeps for 2000 Timer ticks.

- Converts itself back into a NRT-process.

\section{Listing B.22: mrttest8.c}

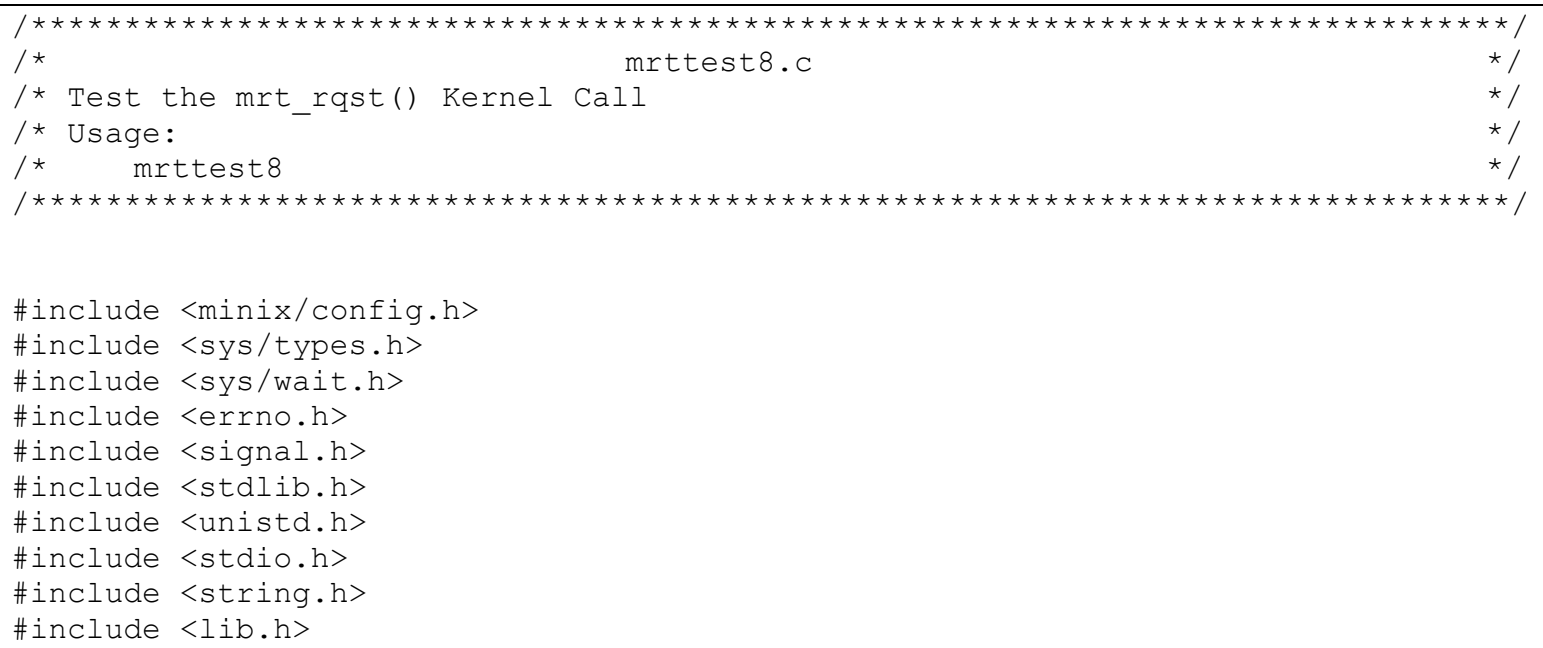




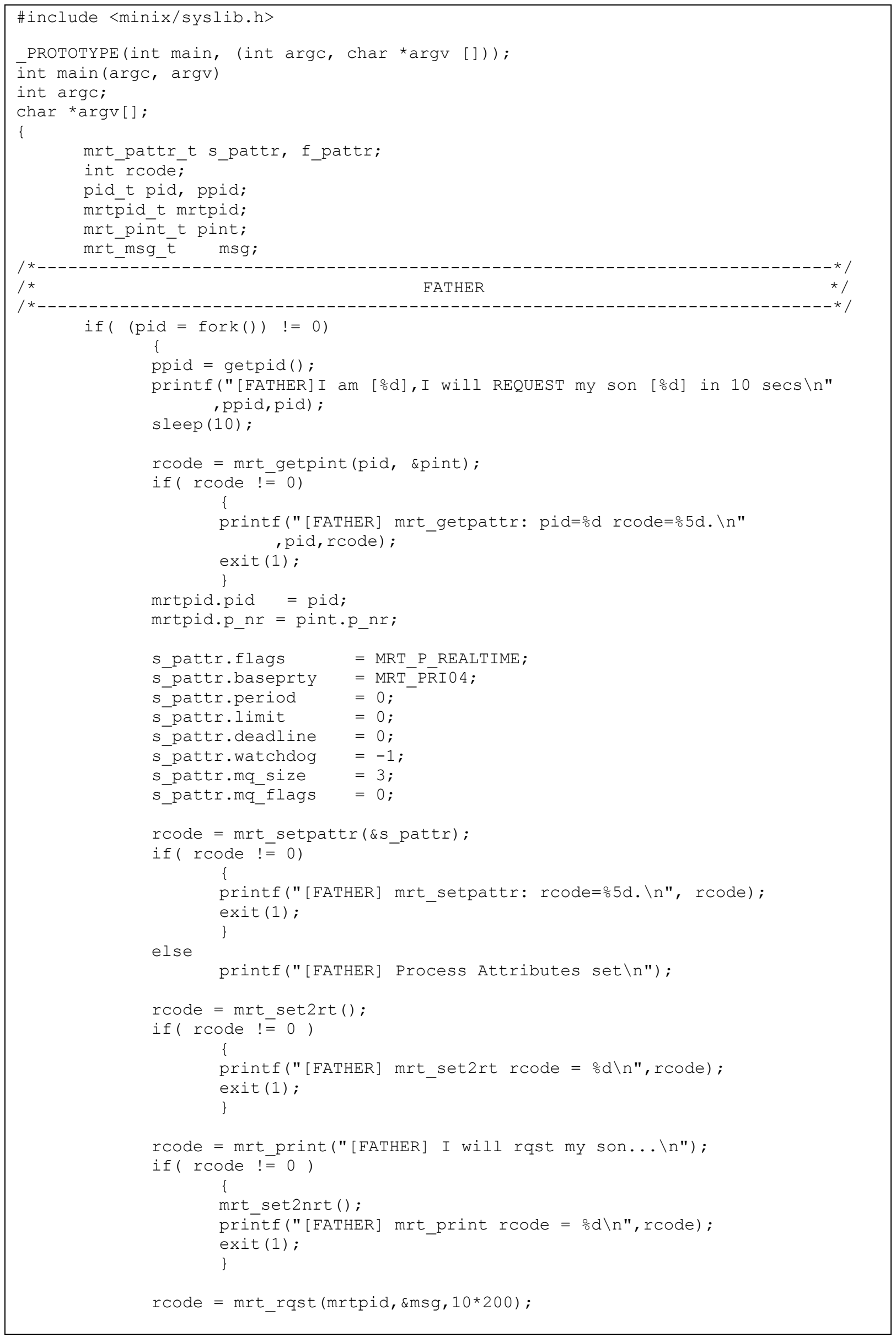




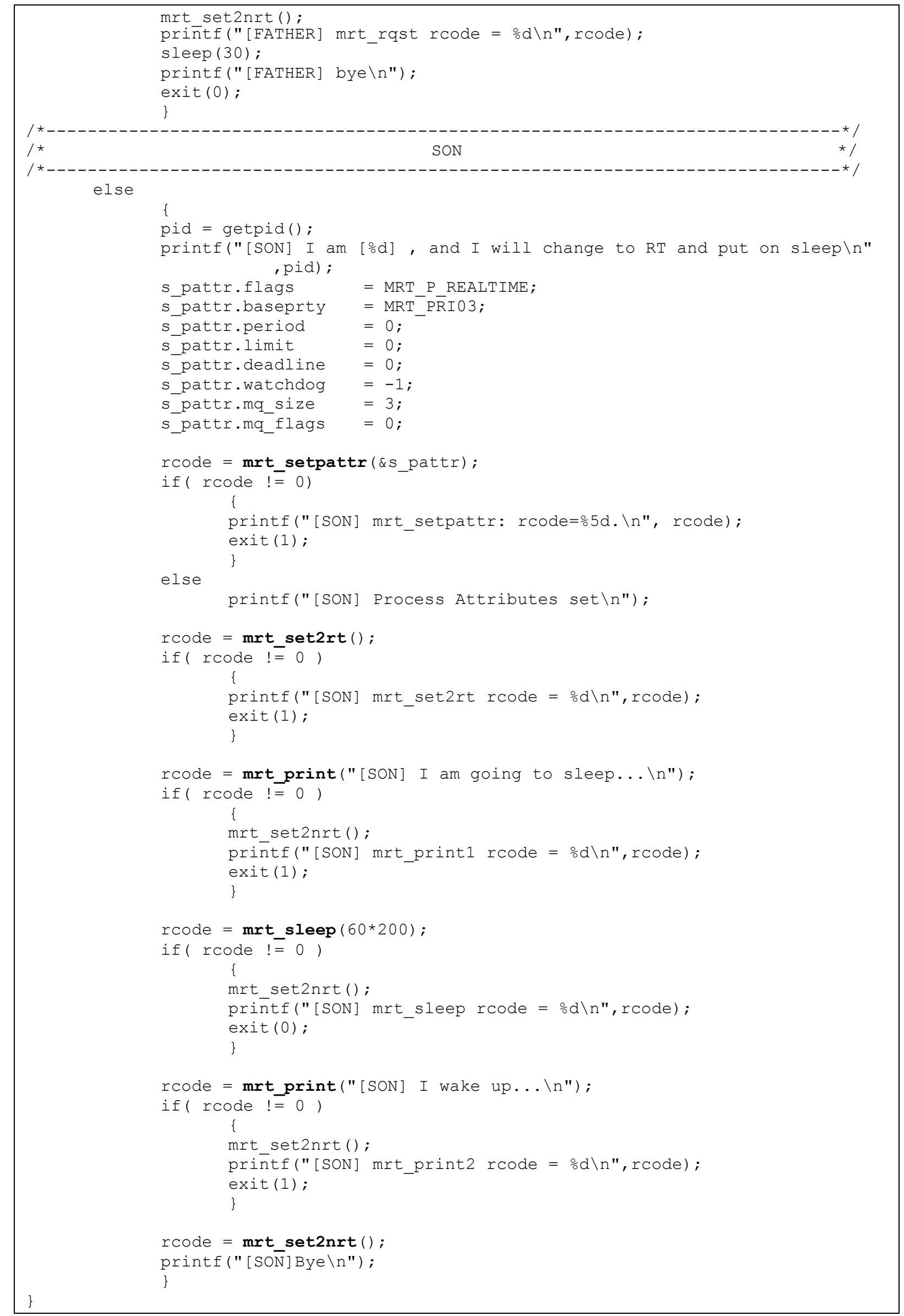




\section{B.23. mrttest8b.c}

This program forks into two processes, the FATHER and the SON:

The FATHER:

- Gets SON's process descriptor internal values to obtain its RT-PID.

- Sets its process descriptor attributes to convert itself into a RT-process.

- Converts itself into a RT-process.

- Prints a text on the system console using mrt_print() Kernel Call.

- Calls mrt_rcv() Kernel Call to receive a message from SON with a timeout of 2000 Timer ticks.

- Converts itself back into a NRT-process.

The $S O N$ :

- Sets its process descriptor attributes to convert itself into a RT-process.

- Converts itself into a RT-process.

- Prints a text on the system console using mrt_print() Kernel Call.

- Sleeps for 2000 Timer ticks.

- Converts itself back into a NRT-process.

\section{Listing B.23: mrttest8b.c}

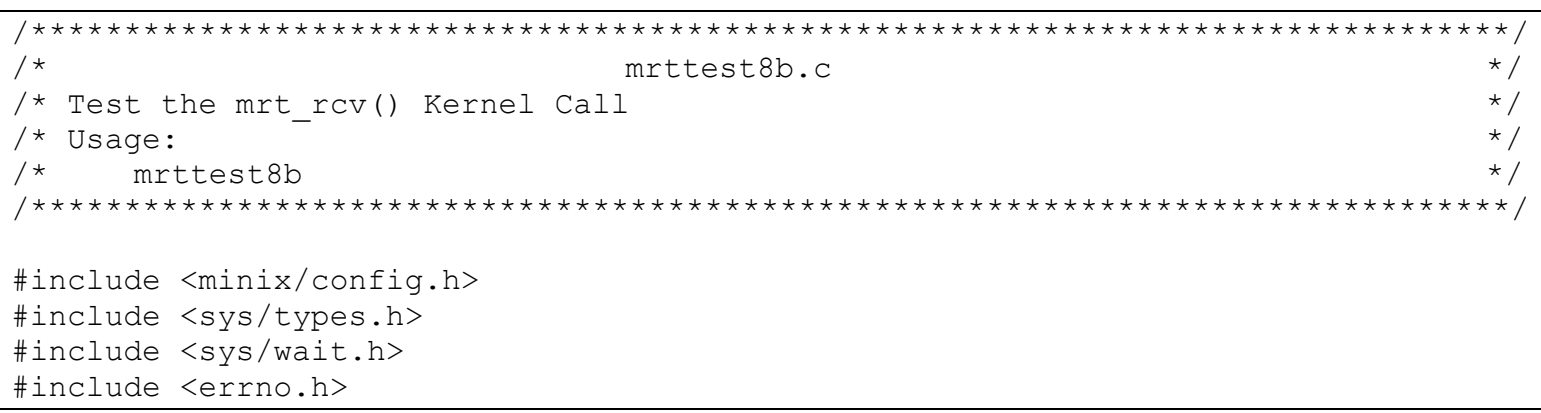




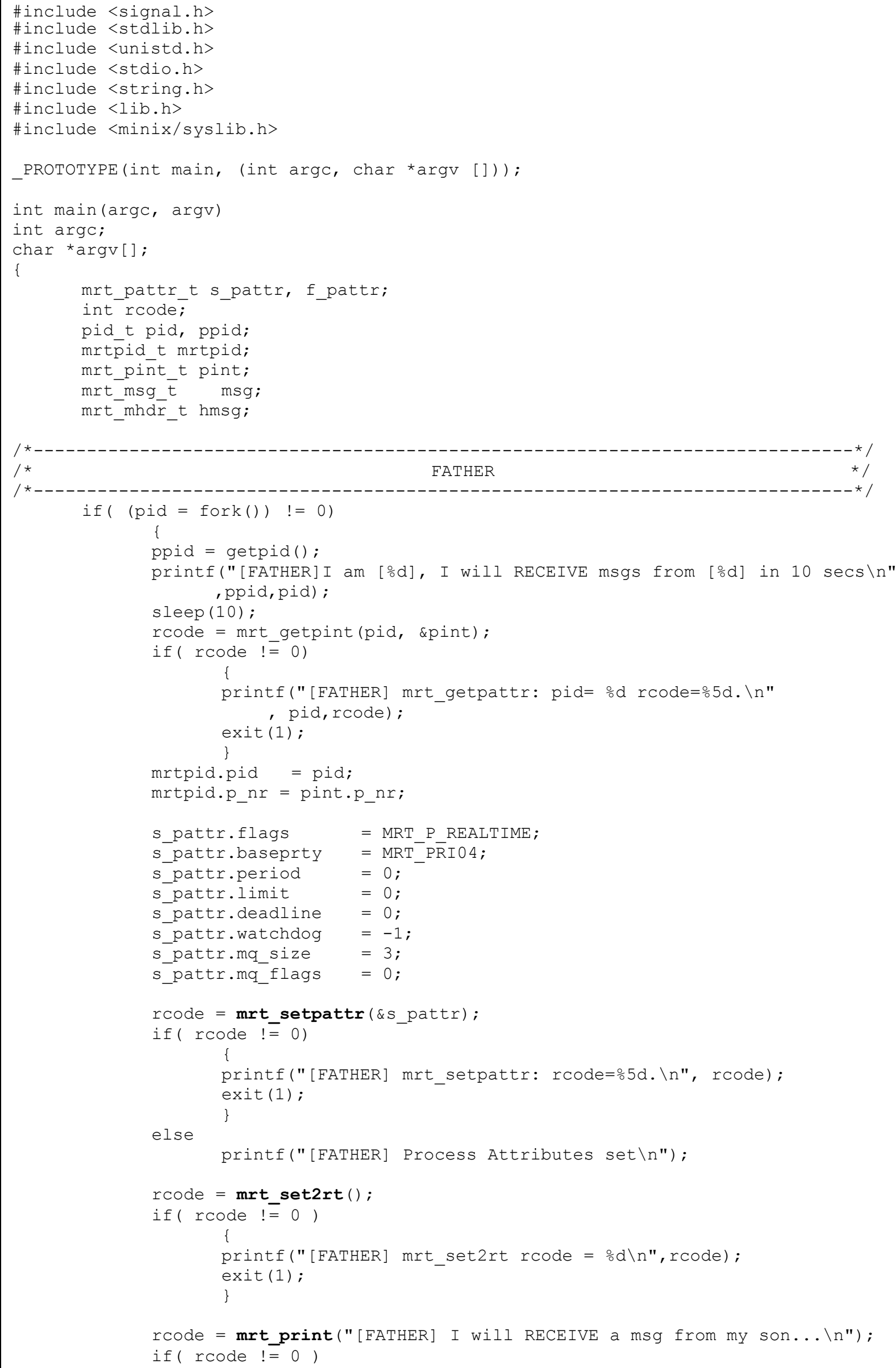




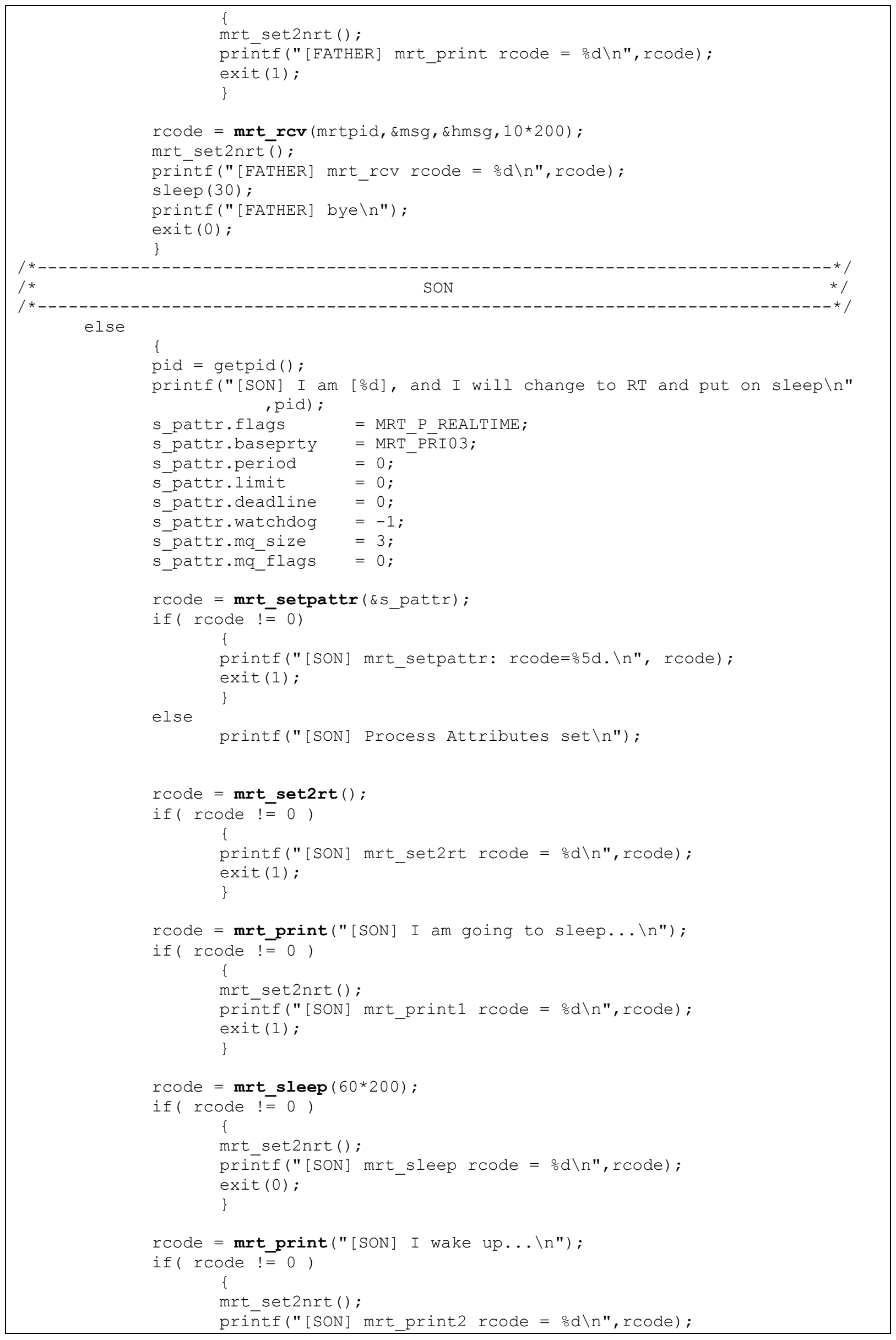




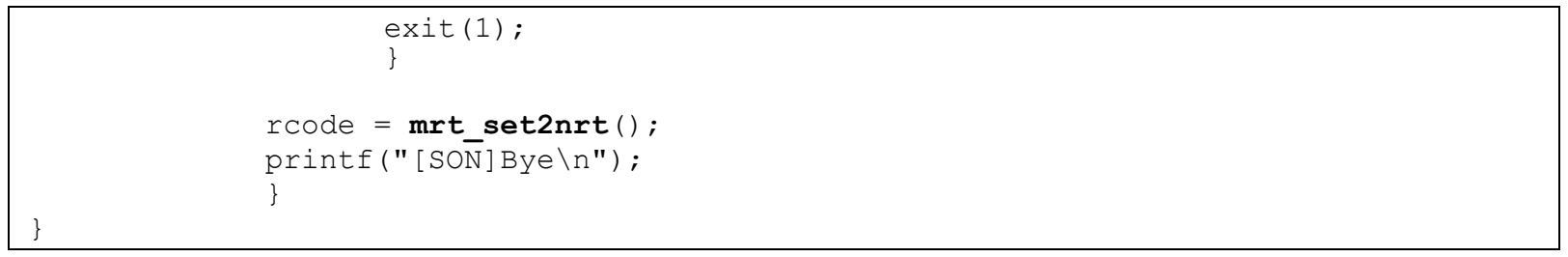

\section{B.24. mrttest8c.c}

This program forks into two processes, the FATHER and the SON:

The FATHER:

- Gets SON's process descriptor internal values to obtain its RT-PID.

- Sets its process descriptor attributes to convert itself into a RT-process.

- Converts itself into a RT-process.

- Loops 10 Times

- Sleeps for 2000 Timer ticks on each loop.

- Send a request message to SON on each loop.

- Prints a text on the system console using mrt print() Kernel Call.

- Converts itself back into a NRT-process.

The SON:

- Sets its process descriptor attributes to convert itself into a RT-process.

- Converts itself into a RT-process.

- Loops forever

- Calls mrt_rcv() Kernel Call to receive a message from FATHER.

- Prints the message content on the system console using mrt print() Kernel Call. 
Listing B.24: mrttest8c.c

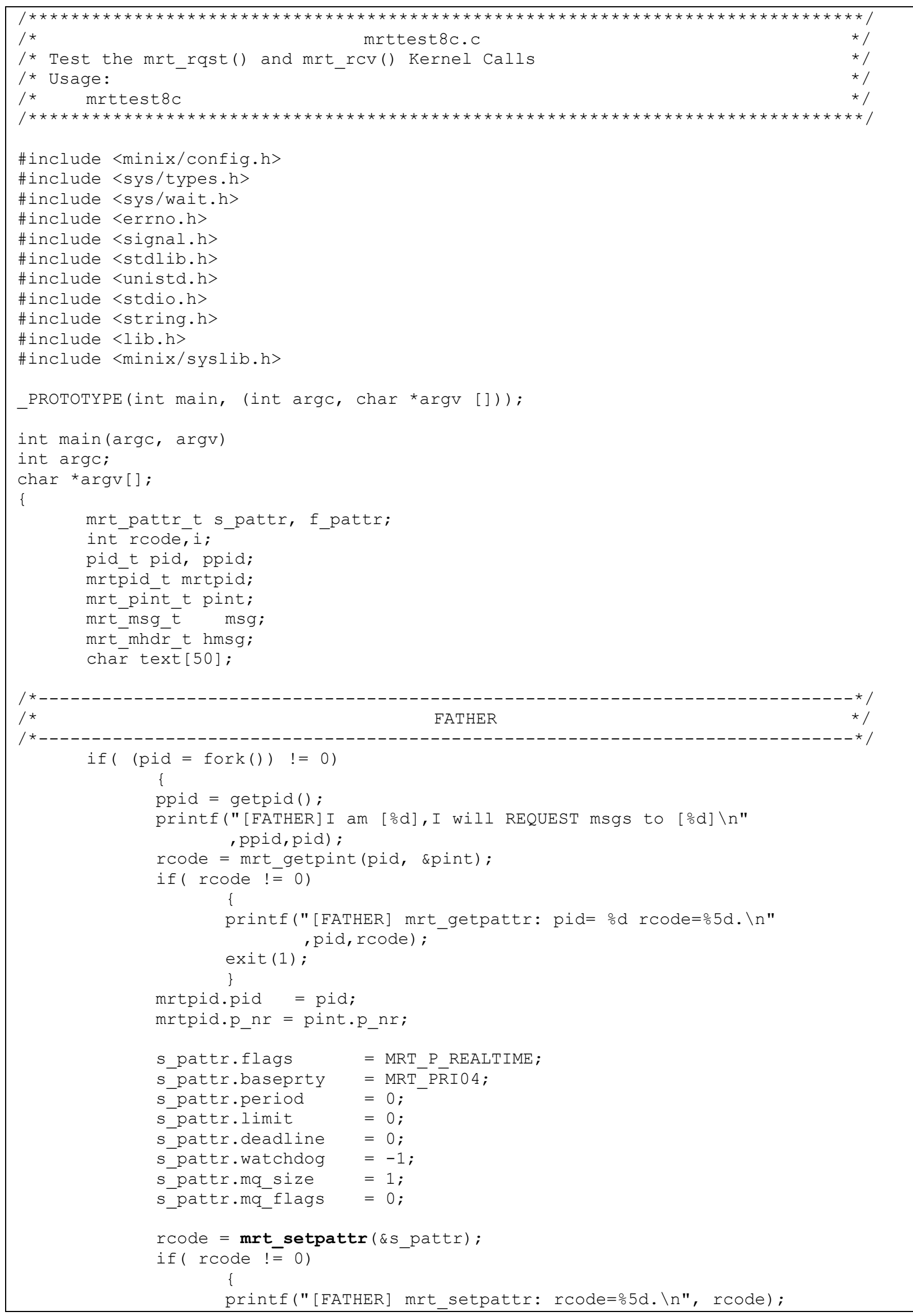




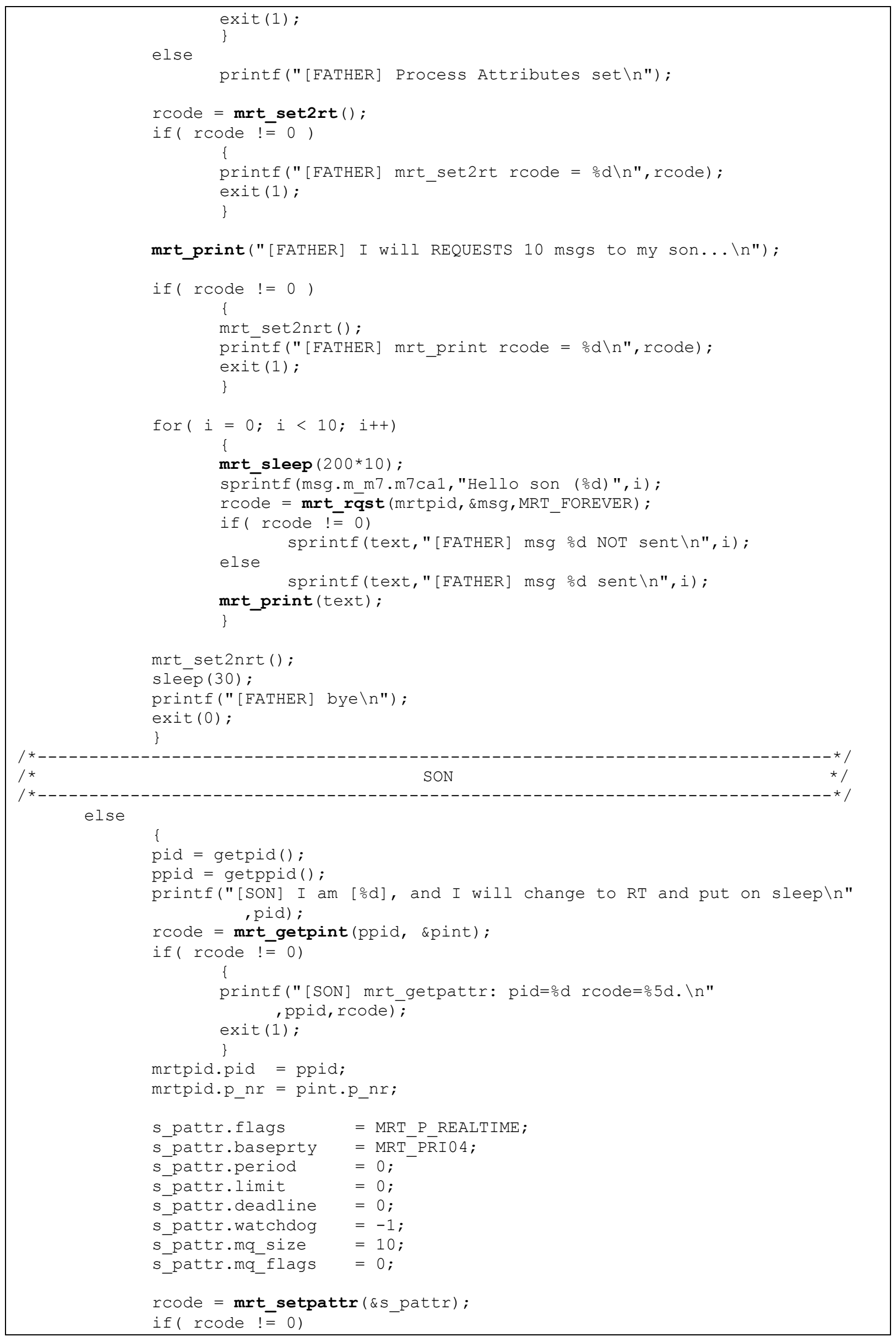




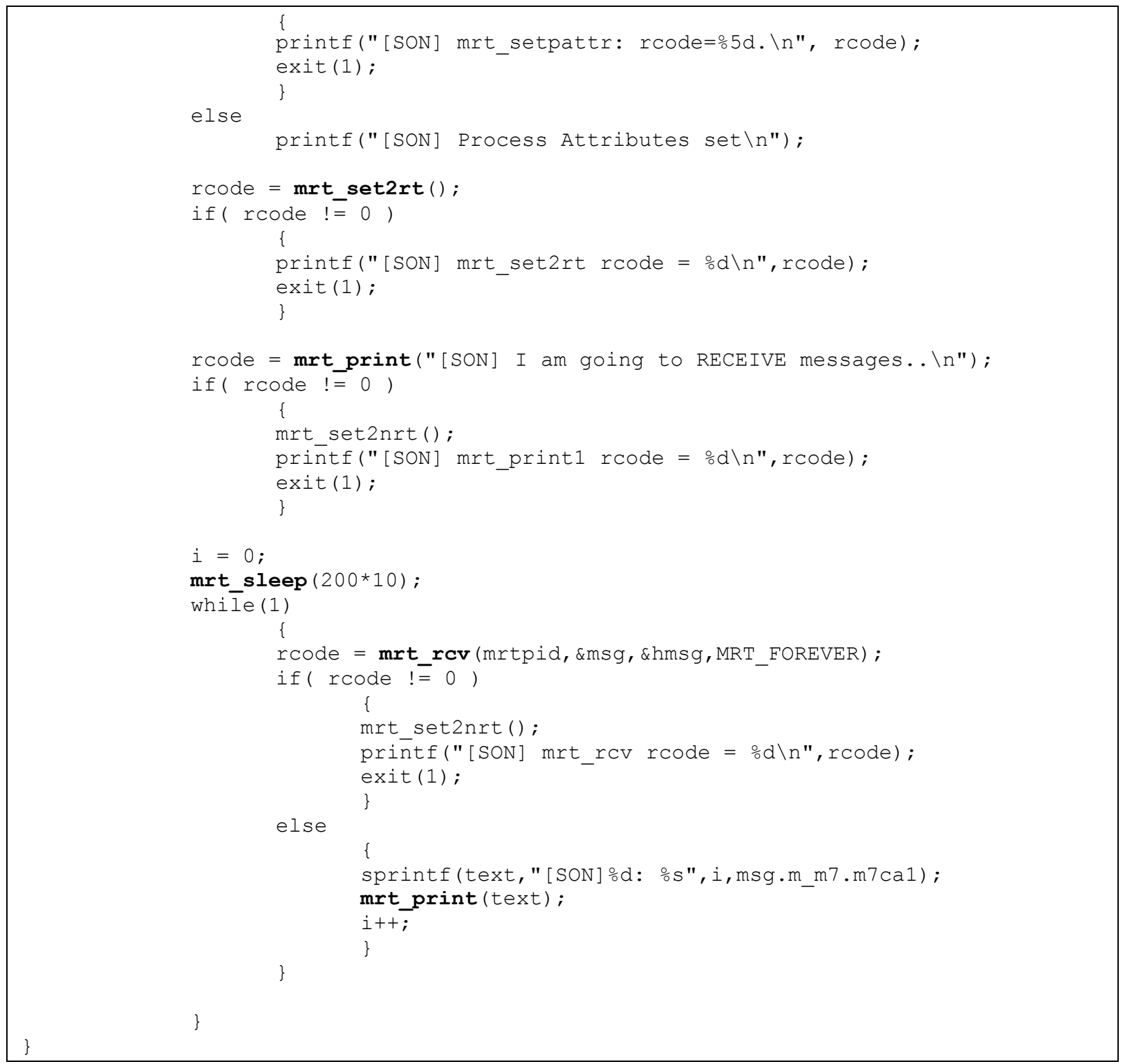

\section{B.25. mrttest8d.c}

This program forks into two processes, the FATHER and the SON:

The FATHER:

- Gets SON's process descriptor internal values to obtain its RT-PID.

- Sets its process descriptor attributes to convert itself into a RT-process.

- Converts itself into a RT-process.

- Loops 10 Times 
- Send an asynchronous request message to SON on each loop.

- Prints a text on the system console using mrt_print() Kernel Call.

- Converts itself back into a NRT-process.

The SON:

- Sets its process descriptor attributes to convert itself into a RT-process.

- Converts itself into a RT-process.

- Loops forever

- Calls mrt_rcv() Kernel Call to receive a message from FATHER.

- Prints the message content on the system console using mrt print) Kernel Call.

Listing B.25: mrttest8d.c

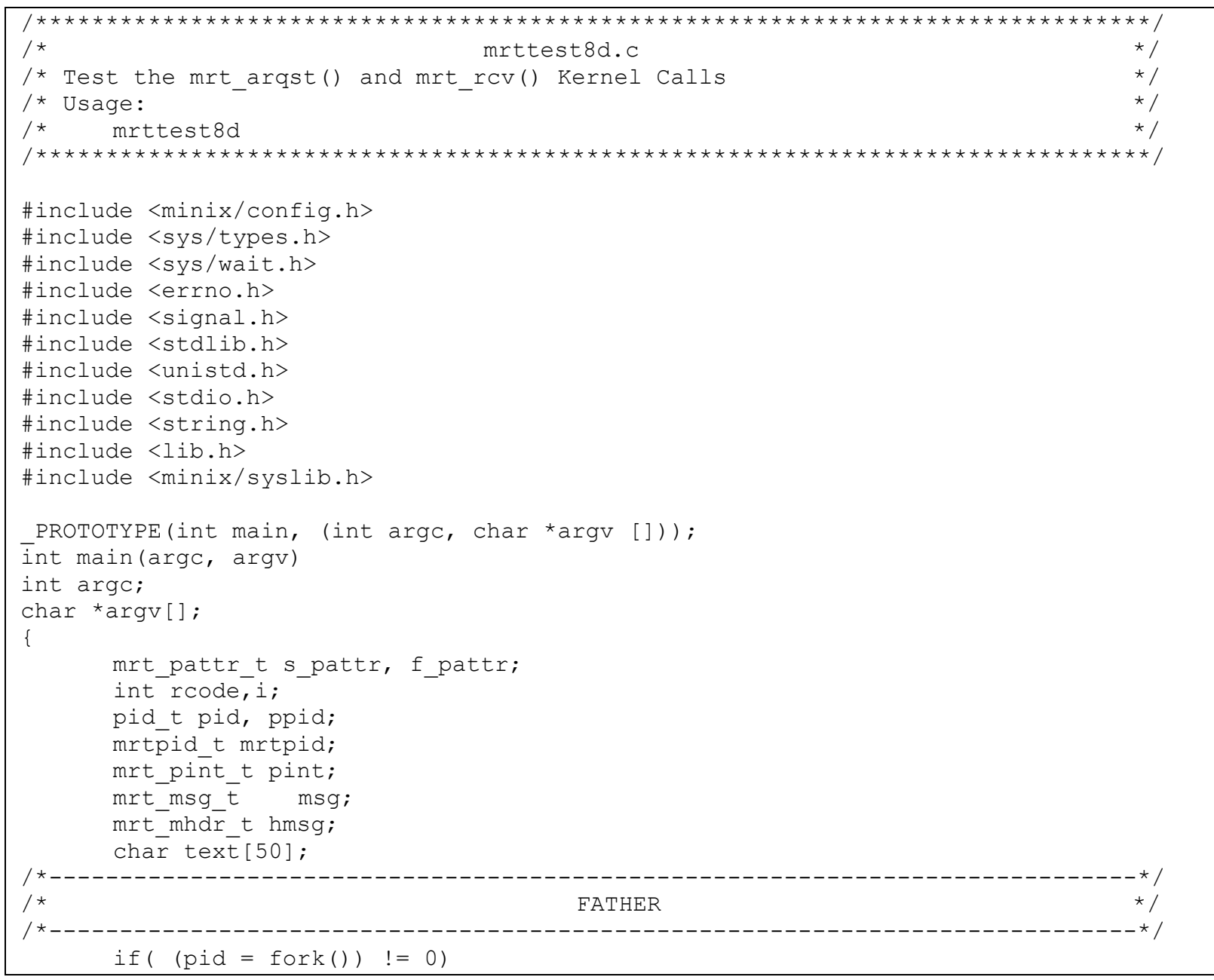




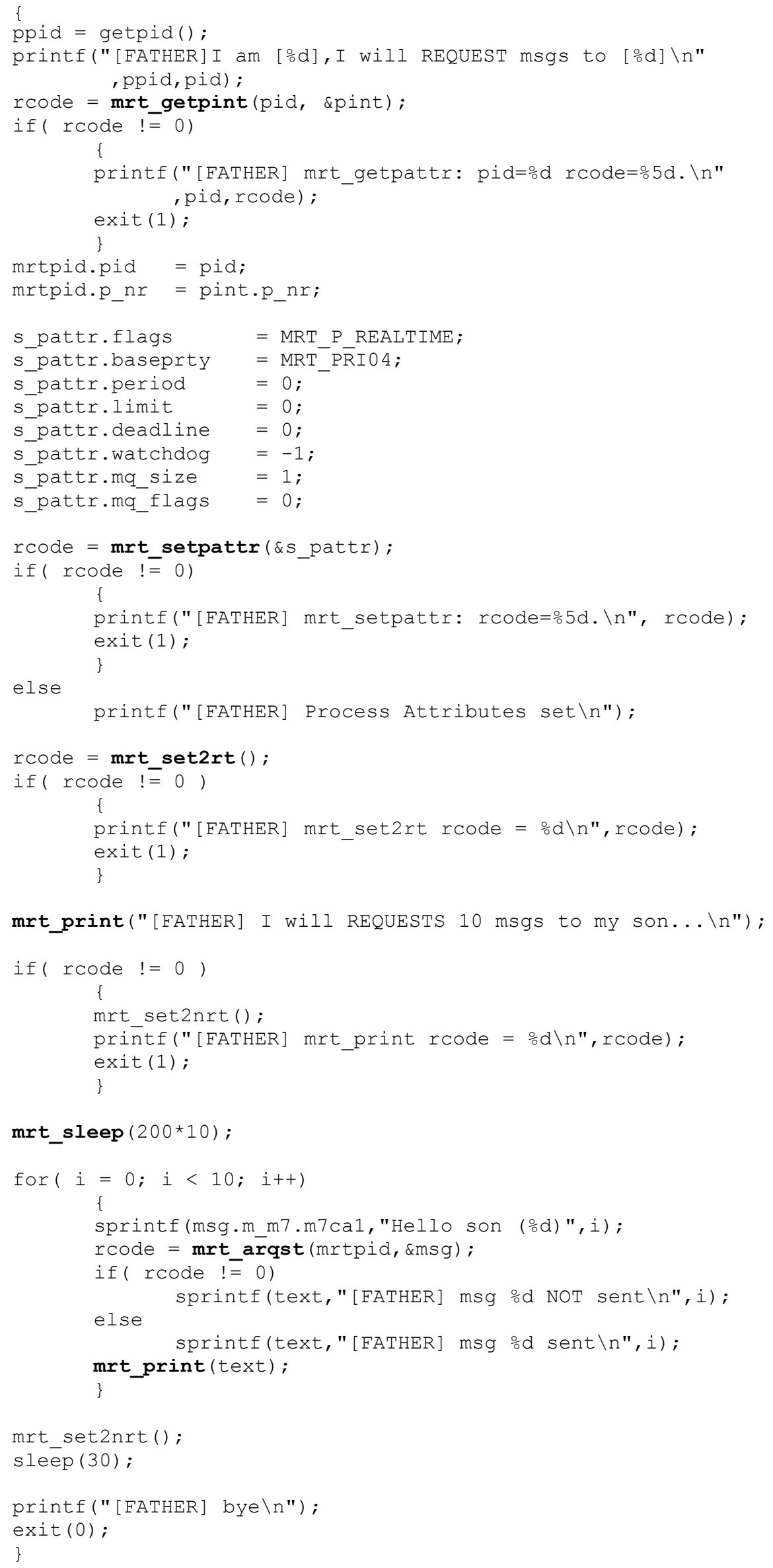




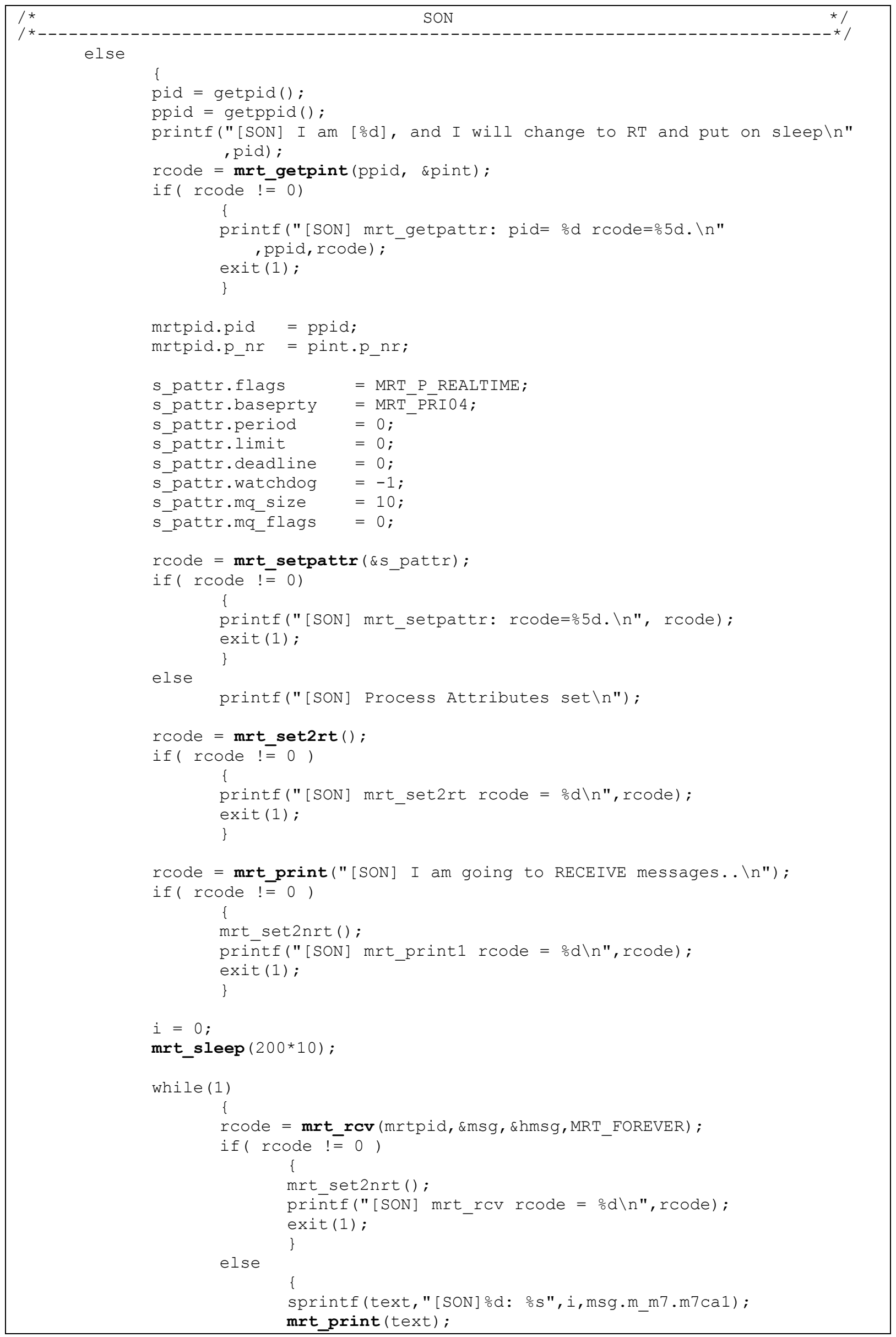




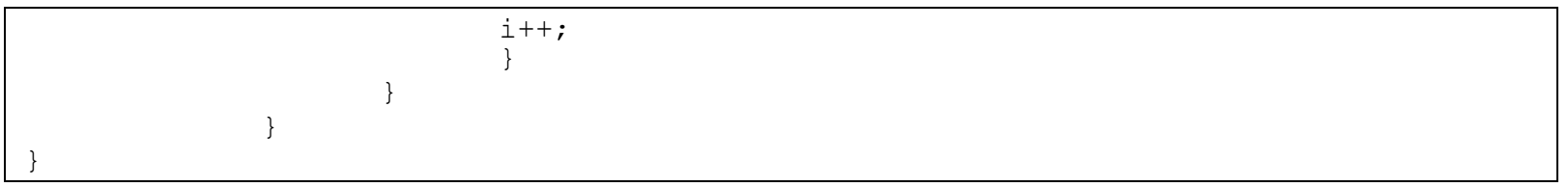

\section{B.26. mrttest8e.c}

This program forks into two processes, the FATHER and the SON:

The FATHER:

- Gets SON's process descriptor internal values to obtain its RT-PID.

- Sets its process descriptor attributes to convert itself into a RT-process.

- Converts itself into a RT-process.

- Sends a request message to SON.

- Calls mrt_rcv() Kernel Call to receive the reply from SON.

- Prints the reply on the system console using mrt print() Kernel Call.

- Converts itself back into a NRT-process.

The SON:

- Sets its process descriptor attributes to convert itself into a RT-process.

- Converts itself into a RT-process.

- Calls mrt_rcv() Kernel Call to receive a message from FATHER.

- Sends a reply message to FATHER.

- Prints the request on the system console using mrt_print() Kernel Call.

- Converts itself back into a NRT-process.

\section{Listing B.26: mrttest8e.c}

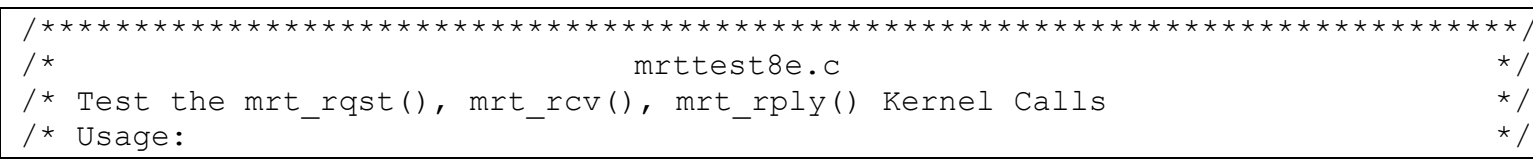




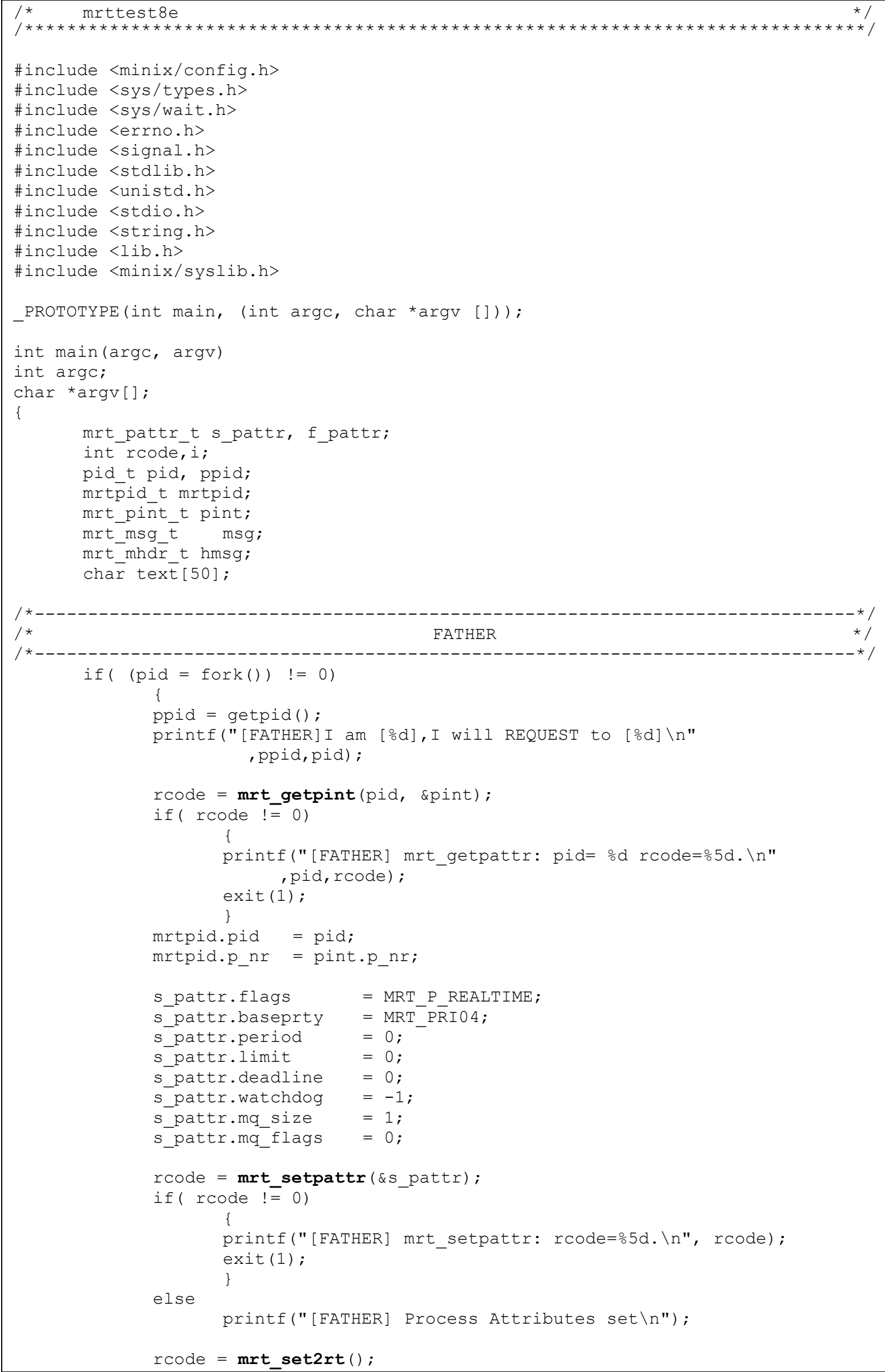




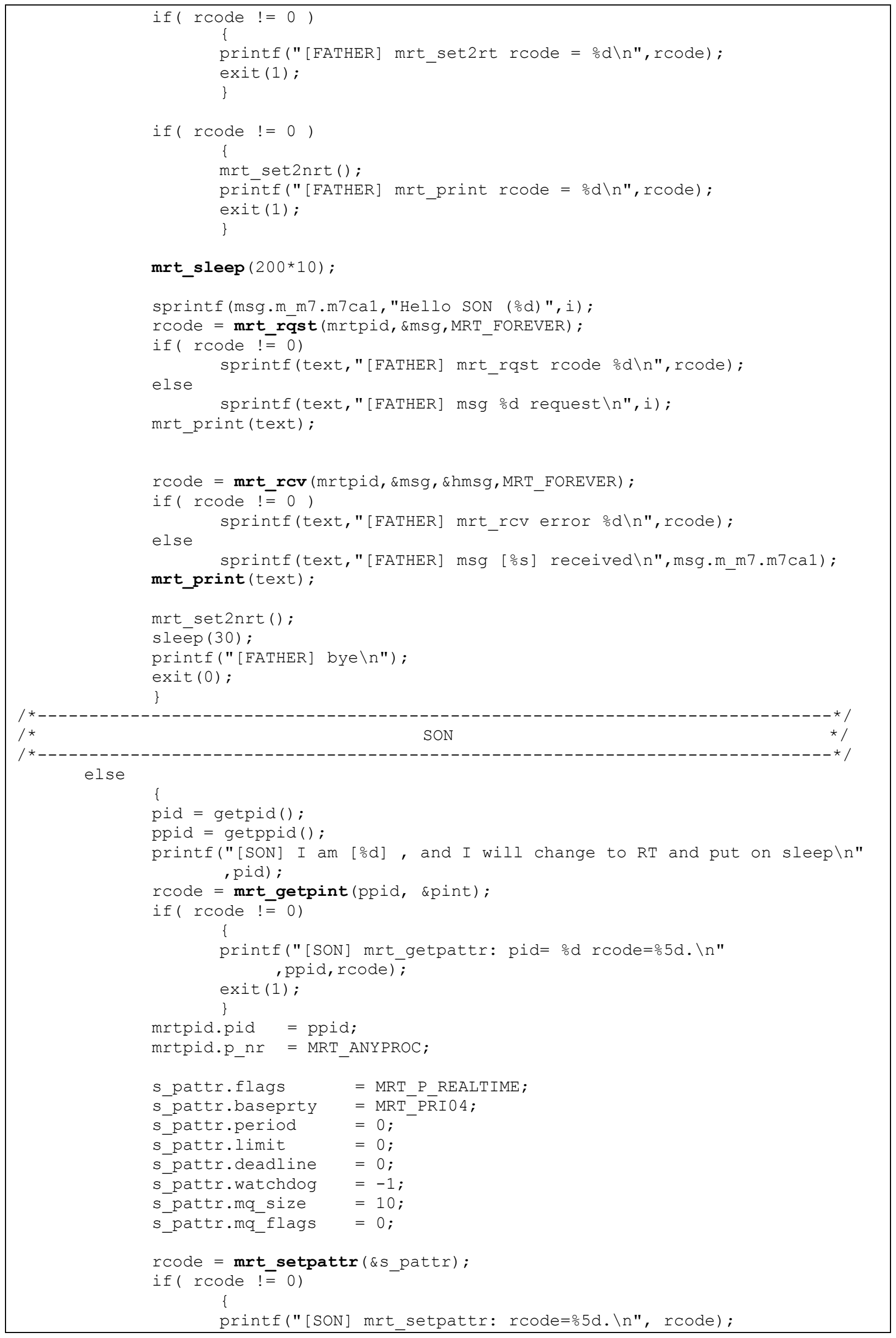




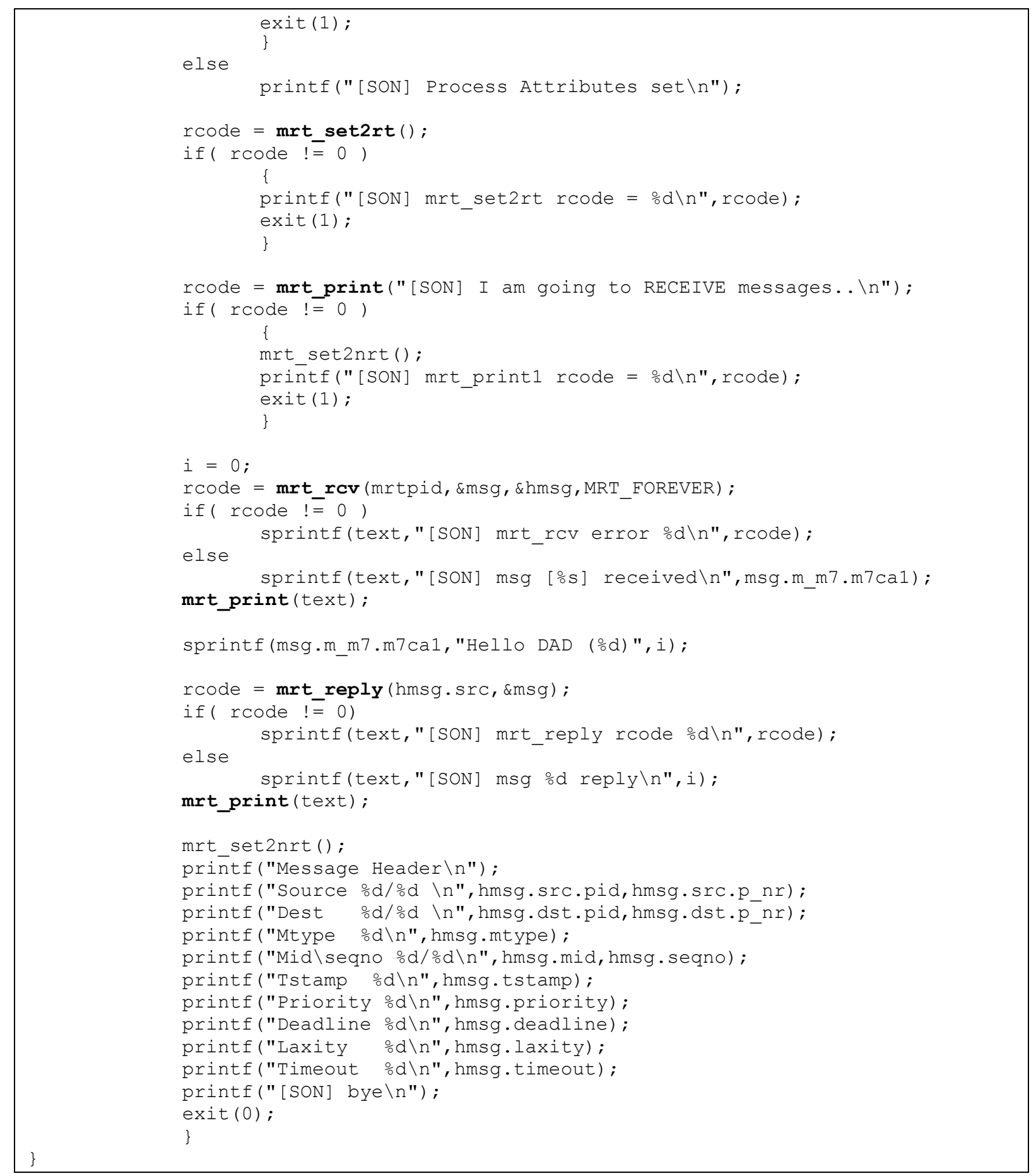

\section{B.27. mrttest9.c}

This program forks into two processes, the FATHER and the SON:

The FATHER:

- Gets SON's process descriptor internal values to obtain its RT-PID. 
- Sets its process descriptor attributes to convert itself into a RT-process.

- Converts itself into a RT-process.

- Loops 5 times

- Sends an up request message to SON on each loop.

- Converts itself back into a NRT-process.

The SON:

- Sets its process descriptor attributes to convert itself into a RT-process.

- Converts itself into a RT-process.

- Loops forever.

- Calls mrt_rcv() Kernel Call to receive a message from FATHER on each loop.

- Prints the request on the system console using mrt_print() Kernel Call on each loop.

\section{Listing B.27: mrttest9.c}

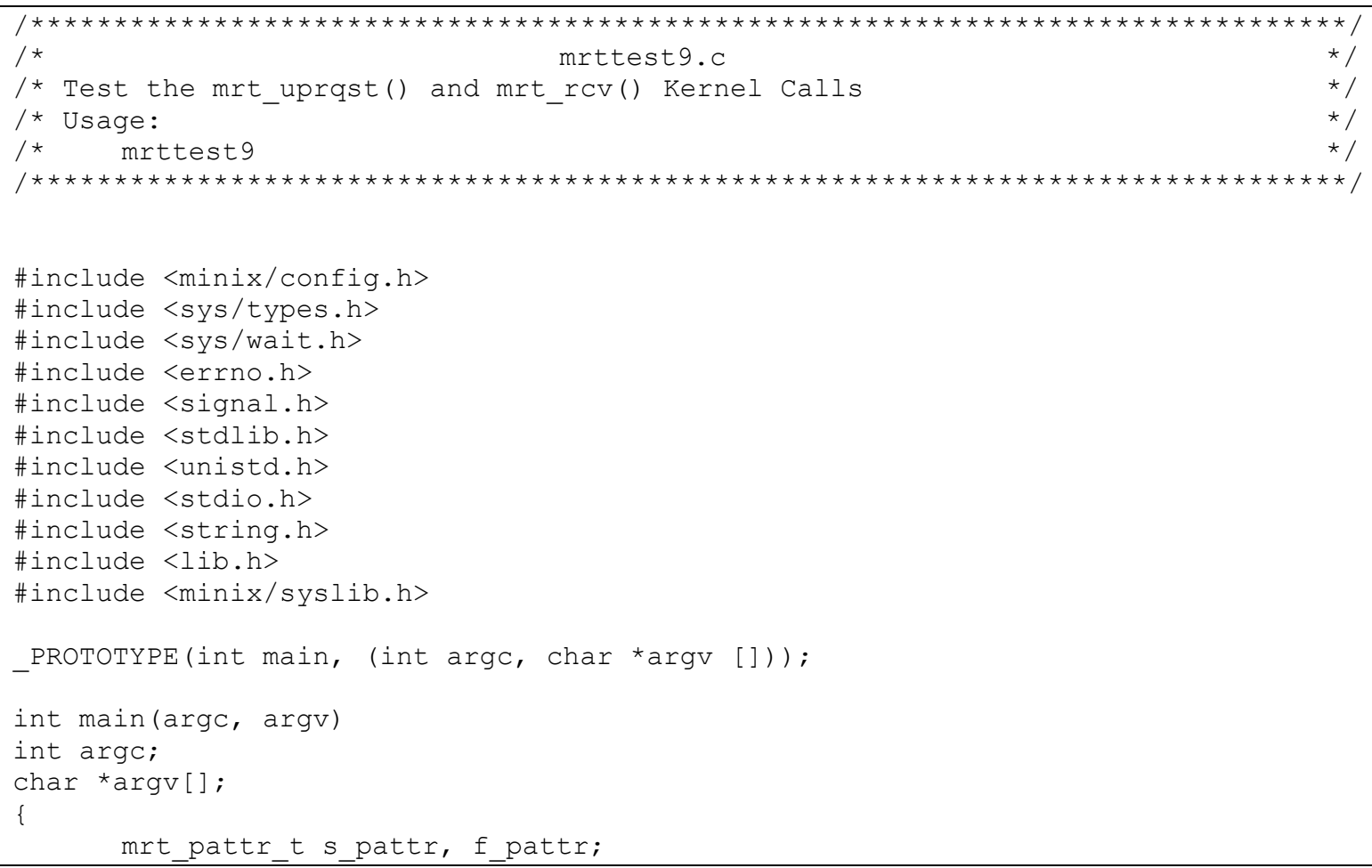




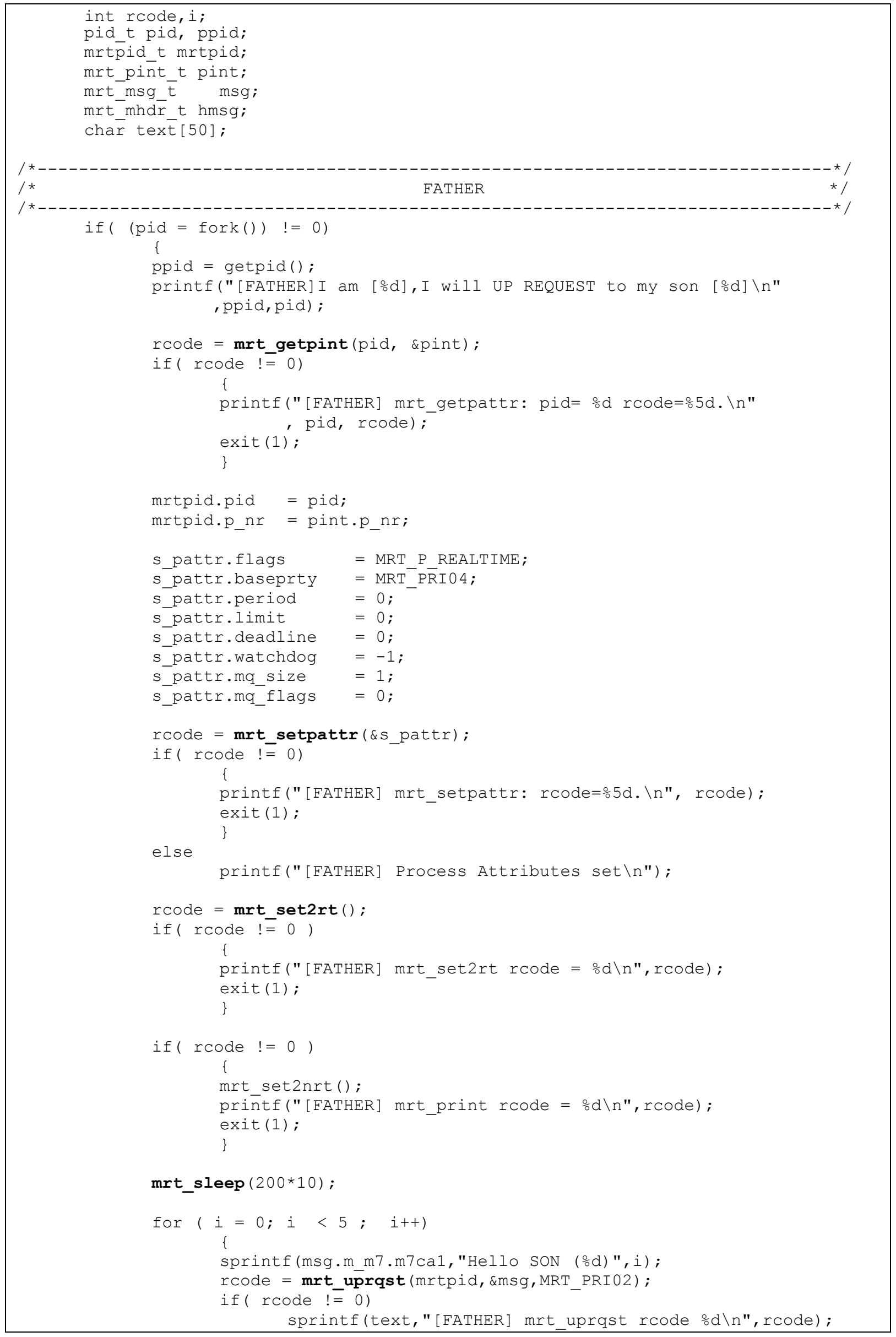




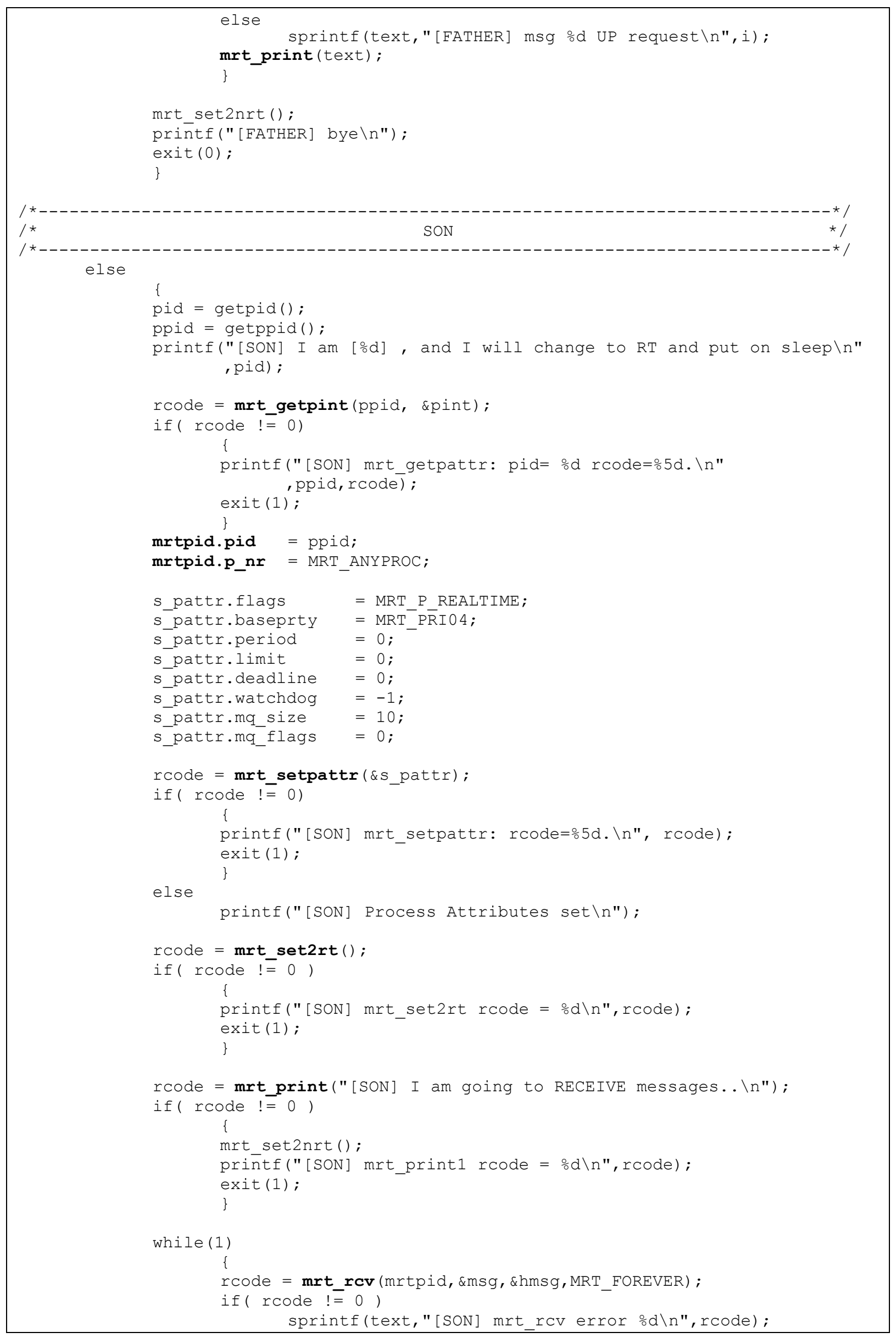




\begin{tabular}{|ll}
\hline & else \\
& mrt_print(text); \\
& \}
\end{tabular}




\section{Appendix C: PERFORMANCE TESTS}

This appendix describe the set of tests carried out on MINIX4RT. The first set that are focused on interrupt service times and Timer services timeliness were performed by Felipe Ieder and Nicolas Cesar as a laboratory practice of Real-Time Operating Systems at Facultad Regional Santa Fe of Universidad Tecnológica Nacional (FRSF-UTN) in May 2004. Because the FRSF-UTN does not have suitable equipment, the methods used for the tests are not rigurous, therefore the results are not accurated.

The set of test on RT-IPC perfomance were performed by the author in December 2005 using an up-to-date kernel.

\section{C.1. Interrupt Service Time}

The interrupt service time tests include different options for interrupt handlers.

- Event Driven Interrupt Handler

- Timer Driven Interrupt Handler

- Software Interrupt Handler

The tests were carried out using three different frequencies of interrupts.(1000, 5000 and $10000[\mathrm{~Hz}])$. The parallel port was used as the source of interrupts for the set of tests.

The equipment used for the test was:

- LUNIX: Intel Pentium III 800MHz, 256 MB RAM, Hard Disk 80GB,Chipset VIA Software: RT Linux with kernel 2.4.21 on Mandrake 9.1. This computer was used as the monitor machine. 
- PAP: IBM Model 370C Notebook, Intel ${ }^{\circledR}$ DX4 $75 \mathrm{MHz}$ on system board, AT Bus, Memory $8 \mathrm{MB}$, Diskette Drive 1.44MB, Hard Disk Drive 540MB 2.5-inch, PCMCIA One Type-III or two Type-II. Software: MINIX4RT (Kernel 28042004). This computer was used as tested machine.

The tests are performed under different kinds of load for the system:

- No load (NOLoad): All background processes that are started at init are killed before the test.

- I/O Diskette Load (DKTLoad): A process access files on a diskette during the test. The diskette interrupt descriptor is Real-Time Timer Driven with a priority lower than the parallel port.

- I/O Disk Load(HDLoad): A process access files on the hard disk during the test.

- CPU Load(CPULoad): A script load the CPU without any I/O operation during the test.

\section{C.1.1. Delay of RTLinux}

Before testing MINIX4RT, a set of tests were carried out over the system that made the measurements. A parallel port loop was made using a DB25 male connector to connect D1 (pin 3) with ACK (pin 10) of the parallel port (see Figure C.1 and Figure C.2).

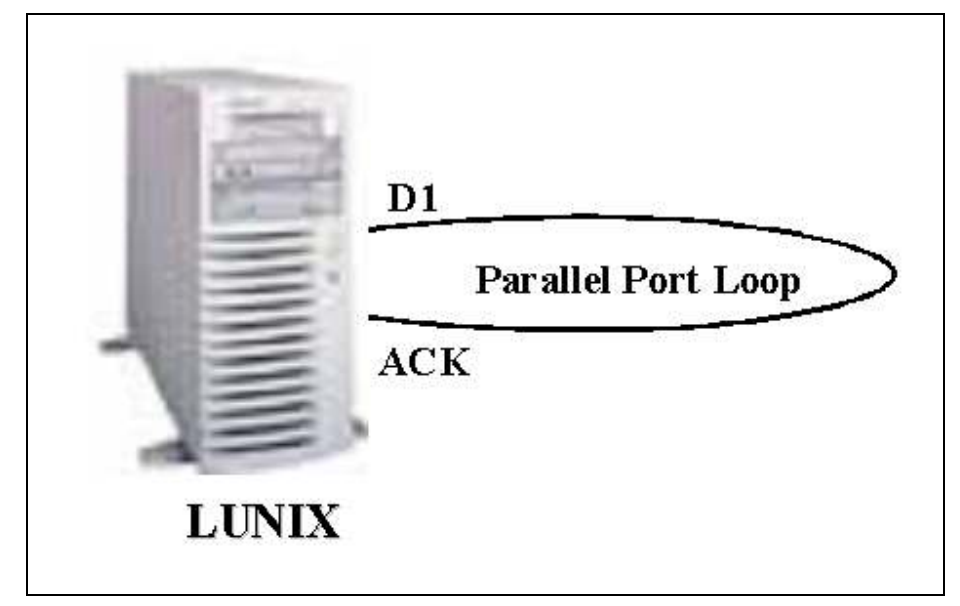

Figure C.1: Delay test of LUNIX (RTLinux) 


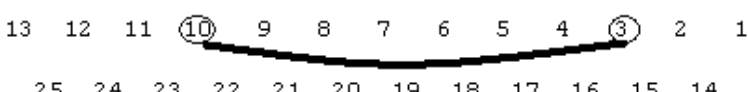

$\begin{array}{llllllllllll}25 & 24 & 23 & 22 & 21 & 20 & 19 & 18 & 17 & 16 & 15 & 14\end{array}$

Figure C.2: DB25 Male Connector

A process in $L U N I X$ machine generate a pulse on pin D1, that generates a parallel port interrupt. The time elapsed beetween the pulse generation and the service of the parallel port interrupt is considered as the delay of the LUNIX machine that must be subtracted from the measurements as is explained en Section C.1.2. The results of $L U N I X$ tests are showed in Table C.1.

Table C.1: Delay of LUNIX (RTLinux).

\begin{tabular}{|c|c|c|c|}
\cline { 2 - 4 } \multicolumn{1}{c|}{} & \multicolumn{3}{c|}{ Frequecy [Hz] } \\
\hline Delay [ns] & $\mathbf{1 0 0 0 0}$ & $\mathbf{5 0 0 0}$ & $\mathbf{1 0 0 0}$ \\
\hline Average & 8379 & 8378 & 8340 \\
\hline Standard Deviation & 670 & 606 & 212 \\
\hline Minimum & 7872 & 7968 & 8032 \\
\hline Maximum & 16576 & 17728 & 11648 \\
\hline
\end{tabular}

\section{C.1.2. Interrupt Service Time Tests of MINIX4RT}

The tests were performed conecting the LUNIX machine with PAP machine (see $\underline{\text { Figure C.3 }}$ ) using a parallel port cable with the pinout detailled in Table C.2.

Table C.2: Testing Cable Pinout

\begin{tabular}{|clc|}
\hline $\begin{array}{c}\text { DB25 Male 1 } \\
\text { PIN }\end{array}$ & DB25 Male 2 \\
2 & PIN \\
3 & $<====>$ & 15 \\
4 & $<====>$ & 13 \\
5 & $<===>$ & 12 \\
6 & $<===>$ & 10 \\
15 & $<===>$ & 11 \\
13 & $<===>$ & 2 \\
12 & $<===>$ & 3 \\
10 & $<===>$ & 4 \\
11 & $<===>$ & 5 \\
25 & $<===>$ & 6 \\
& & 25 \\
\hline
\end{tabular}


The following is the sequence of events of the tests:

- $\quad L U N I X$ generates a pulse on a parallel port Data Bit (D3) that produces a parallel port interrupt on $P A P(\mathrm{ACK})$.

- The parallel port interrupt handler of $P A P$ generates a pulse pulse on a parallel port Data Bit (D3) that produces an interrupt on LUNIX (ACK).

The time elapsed beetween the pulse generation on LUNIX and the parallel port interrupt service in $L U N I X$ is considered as the delay of the LUNIX machine plus the MINIX4RT interrupt service time.

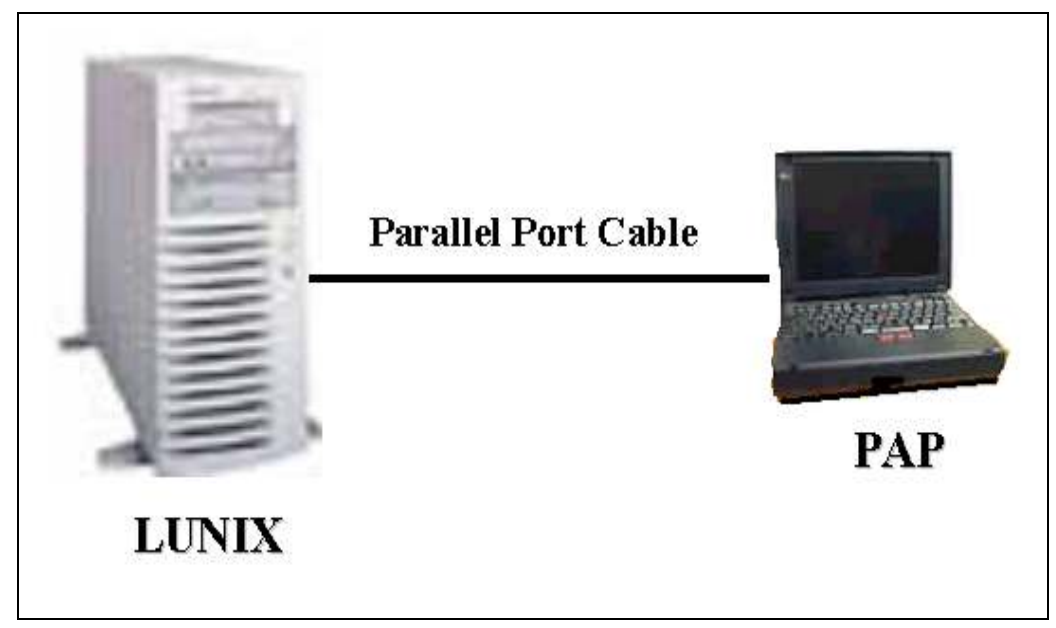

Figure C.3: Interrupt Service Time Tests of MINIX4RT

\section{C.1.3. Interrupt Service Time of Software Interrupts}

This section shows the tests results of Interrupt Service Time for Software interrupts. The RT-interrupt handler triggers a Software Interrupt that generates the response pulse on the parallel port.

Table C.4: Interrupt Service Time of Software Interrupts for 10000 [Hz]

\begin{tabular}{|c|c|c|c|c|}
\hline Delay [ns] & DKTLoad & HDLoad & CPULoad & NOLoad \\
\hline Average & 41841 & 42777 & 43752 & 40974 \\
\hline Standard Deviation & 6594 & 7948 & 8027 & 8104 \\
\hline Minimum & 10112 & 30528 & 30368 & 30528 \\
\hline Maximum & 100320 & 105920 & 117569 & 151456 \\
\hline
\end{tabular}


Table C.5: Interrupt Service Time of Software Interrupts for 5000 [Hz]

\begin{tabular}{|c|c|c|c|c|}
\hline Delay [ns] & DKTLoad & HDLoad & CPULoad & NOLoad \\
\hline Average & 33085 & 42916 & 38622 & 43732 \\
\hline Standard Deviation & 3135 & 4030 & 2759 & 4216 \\
\hline Minimum & 30592 & 31552 & 33152 & 30624 \\
\hline Maximum & 69792 & 87200 & 58976 & 88288 \\
\hline
\end{tabular}

Table C.6: Interrupt Service Time of Software Interrupts for 1000 [Hz]

\begin{tabular}{|c|c|c|c|c|}
\hline Delay [ns] & DKTLoad & HDLoad & CPULoad & NOLoad \\
\hline Average & 38319 & 44443 & 39652 & 35986 \\
\hline Standard Deviation & 6865 & 7523 & 2582 & 6534 \\
\hline Minimum & 30688 & 30848 & 35712 & 30688 \\
\hline Maximum & 62304 & 59584 & 51008 & 87264 \\
\hline
\end{tabular}

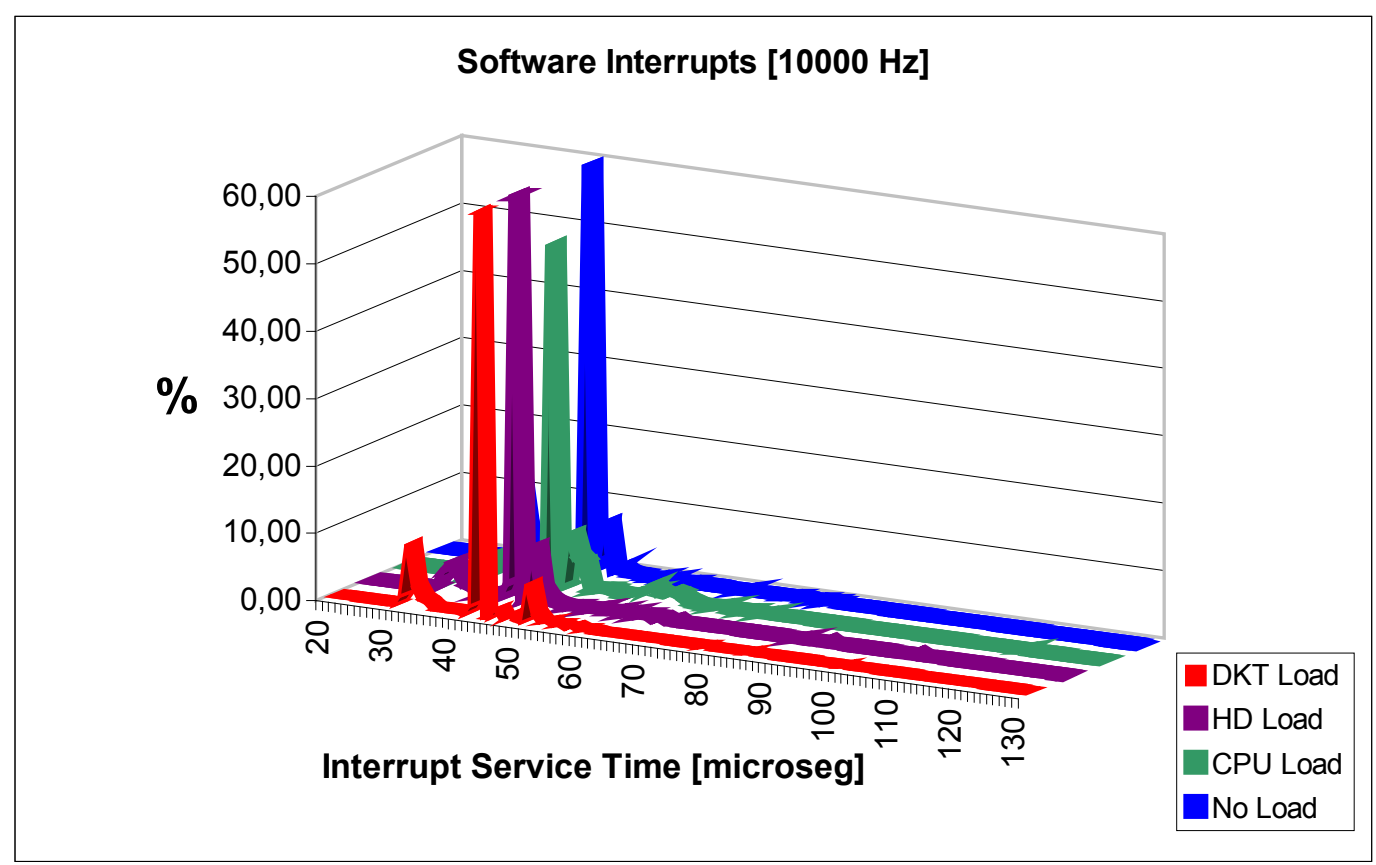

Figure C.4: Interrupt Service Time for Software Interrupts for 10000 [Hz] 


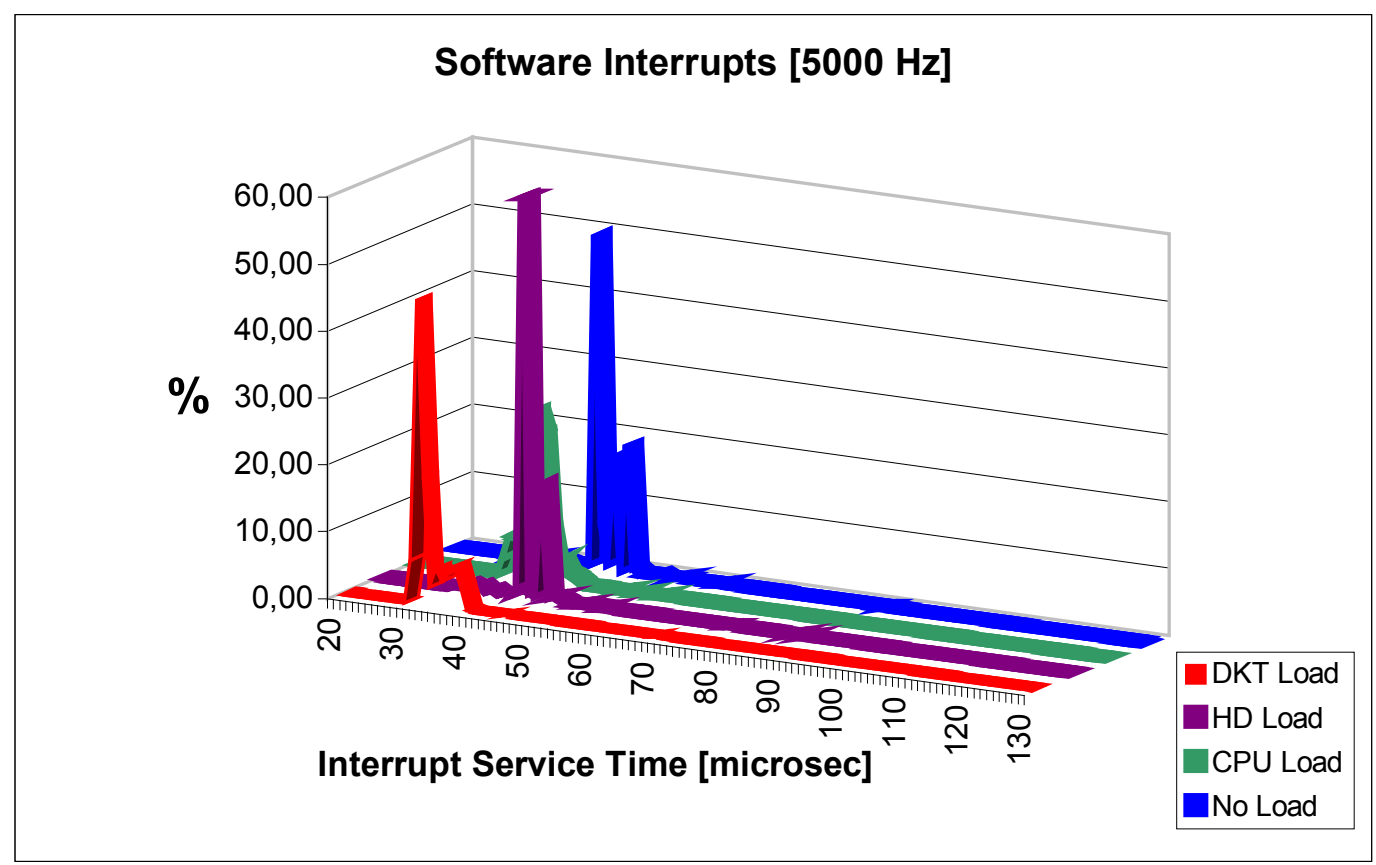

Figure C.5: Interrupt Service Time for Software Interrupts for 5000 [Hz]

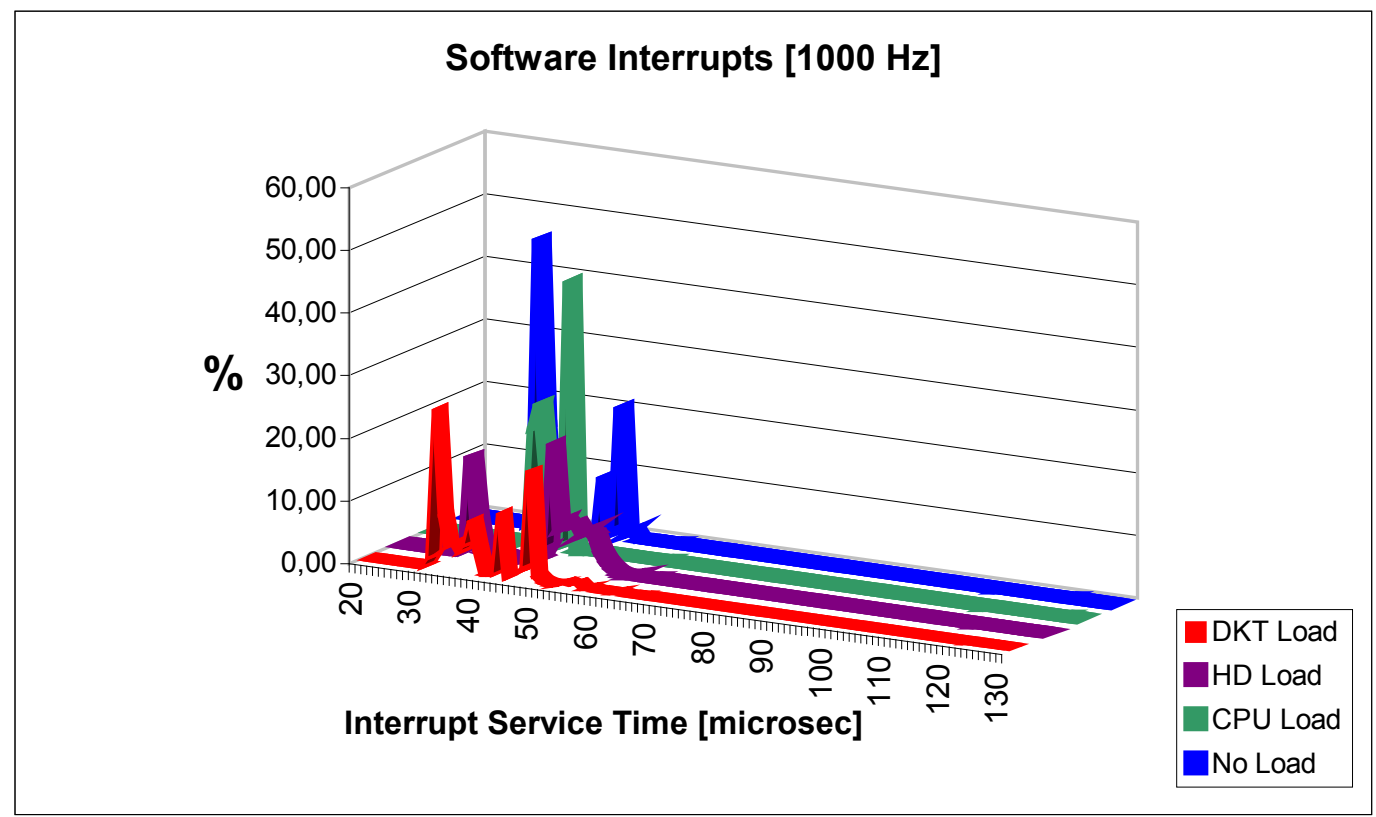

Figure C.6: Interrupt Service Time for Software Interrupts for 1000 [Hz]

\section{C.1.4. Interrupt Service Time of Event-Driven Interrupts}

This section describe the tests results of Interrupt Service Time for Event-Driven interrupts. The RT-interrupt handler generates the response pulse on the parallel port. 
Table C.7: Interrupt Service Time of Event-Driven Interrupts for 10000 [Hz]

\begin{tabular}{|c|c|c|c|c|}
\hline Delay [ns] & DKTLoad & HDLoad & CPULoad & NOLoad \\
\hline Average & 26395 & 38525 & 39909 & 37556 \\
\hline Standard Deviation & 8709 & 9239 & 9309 & 9786 \\
\hline Minimum & 21888 & 21888 & 21856 & 21376 \\
\hline Maximum & 100352 & 89632 & 122816 & 55904 \\
\hline
\end{tabular}

Table C.8: Interrupt Service Time of Event-Driven Interrupts for 5000 [Hz]

\begin{tabular}{|c|c|c|c|c|}
\hline Delay [ns] & DKTLoad & HDLoad & CPULoad & NOLoad \\
\hline Average & 38685 & 41094 & 41706 & 40368 \\
\hline Standard Deviation & 8303 & 6131 & 5355 & 6857 \\
\hline Minimum & 21824 & 21888 & 23168 & 21536 \\
\hline Maximum & 75392 & 82560 & 83424 & 52608 \\
\hline
\end{tabular}

Table C.9: Interrupt Service Time of Event-Driven Interrupts for 1000 [Hz]

\begin{tabular}{|c|c|c|c|c|}
\hline Delay [ns] & DKTLoad & HDLoad & CPULoad & NOLoad \\
\hline Average & 31092 & 33790 & 33506 & 29895 \\
\hline Standard Deviation & 10294 & 10445 & 9100 & 10284 \\
\hline Minimum & 21824 & 22144 & 23232 & 22144 \\
\hline Maximum & 69664 & 90912 & 92896 & 86464 \\
\hline
\end{tabular}

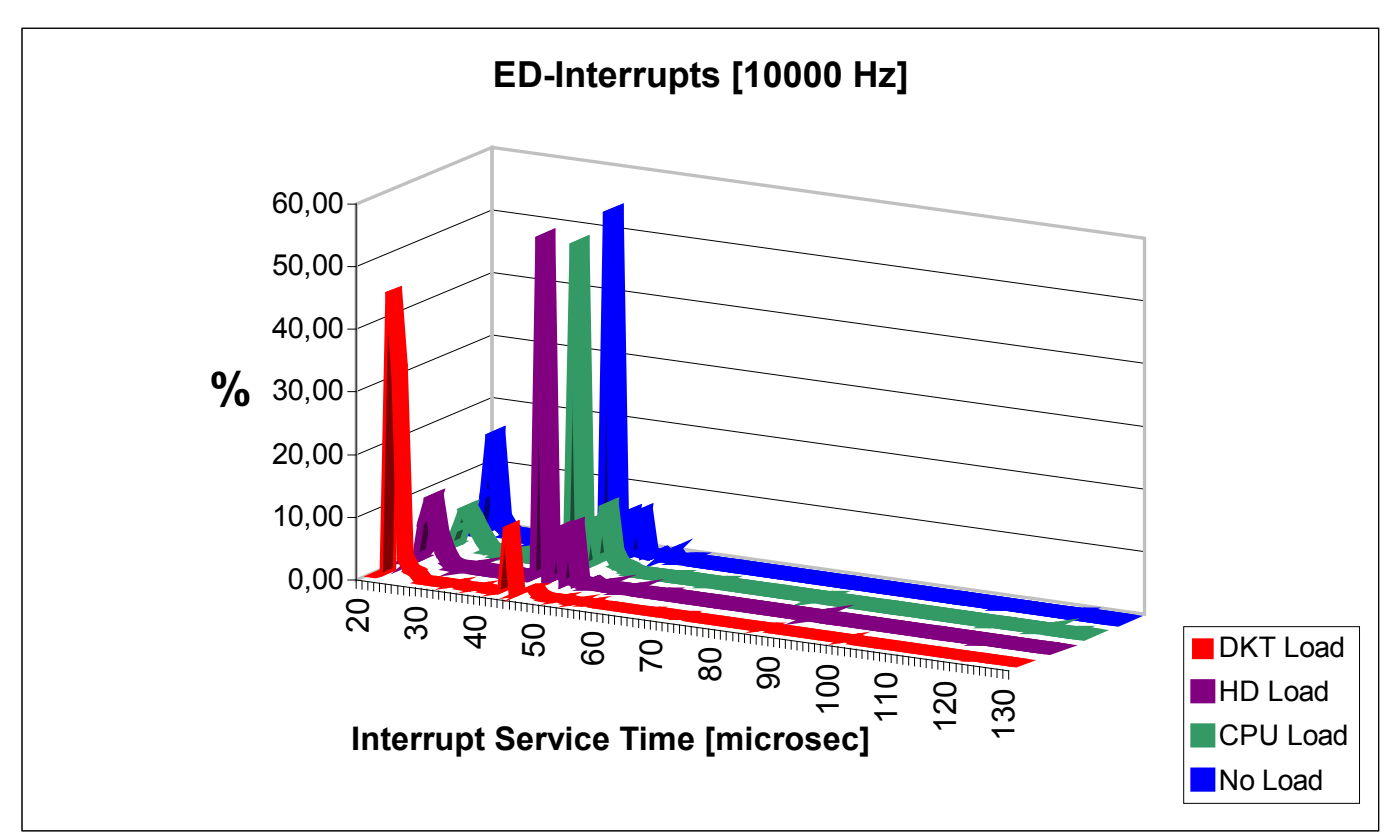

Figure C.7: Interrupt Service Time for ED-Interrupts [10000 Hz] 


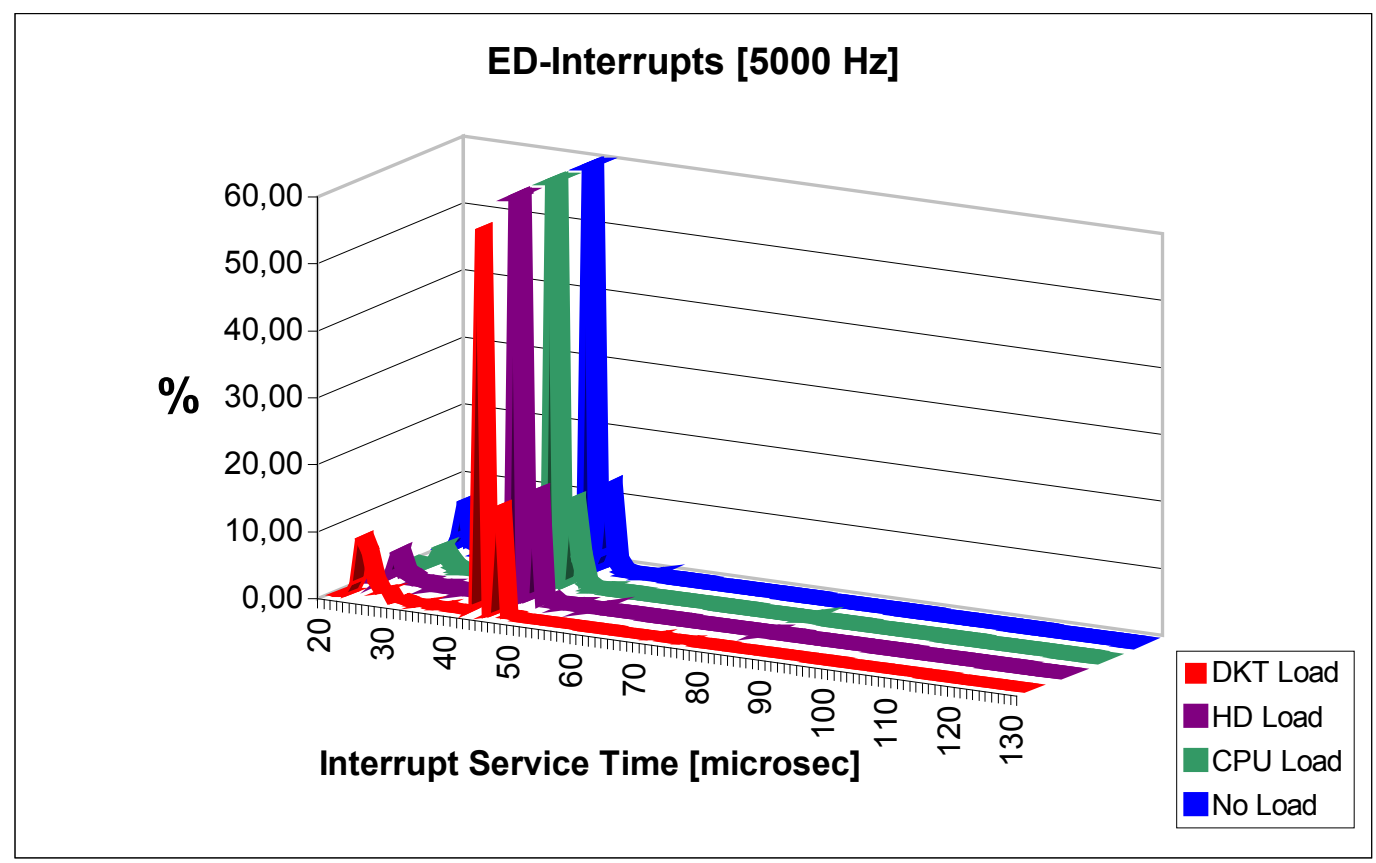

Figure C.8: Interrupt Service Time for ED-Interrupts [5000 Hz]

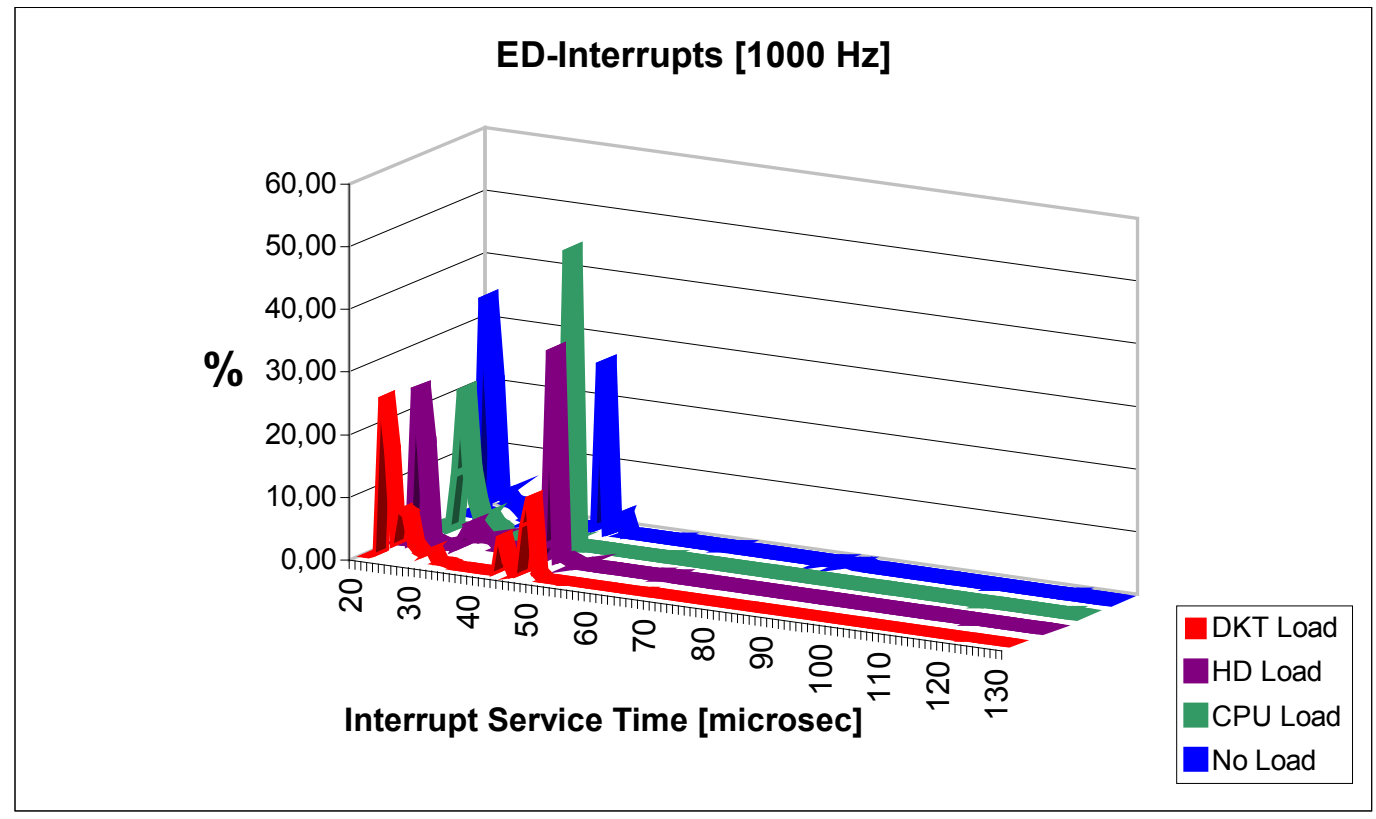

Figure C.9: Interrupt Service Time for ED-Interrupts [1000 Hz]

\section{C.1.5. Interrupt Service Time of Timer-Driven Interrupts}

This section describe the tests results Interrupt Service Time of Timer-Driven interrupts. In this tests, the RT-interrupt handler sets the interrupt descriptor to be executed 
in the next Timer Interrupt. The TD-Interrupt descriptor generates the response pulse on the parallel port. The Timer interrupt frequency was $5000[\mathrm{~Hz}]$.

Table C.10: Interrupt Service Time of Timer-Driven Interrupts for 1000 [Hz]

\begin{tabular}{|c|c|c|c|c|}
\hline Delay [ns] & DKTLoad & HDLoad & CPULoad & NOLoad \\
\hline Average & 532139 & 462369 & 322101 & 256622 \\
\hline Standard Deviation & 24242 & 27099 & 21207 & 23484 \\
\hline Minimum & 488544 & 412416 & 288832 & 215616 \\
\hline Maximum & 610688 & 540256 & 378624 & 314464 \\
\hline
\end{tabular}

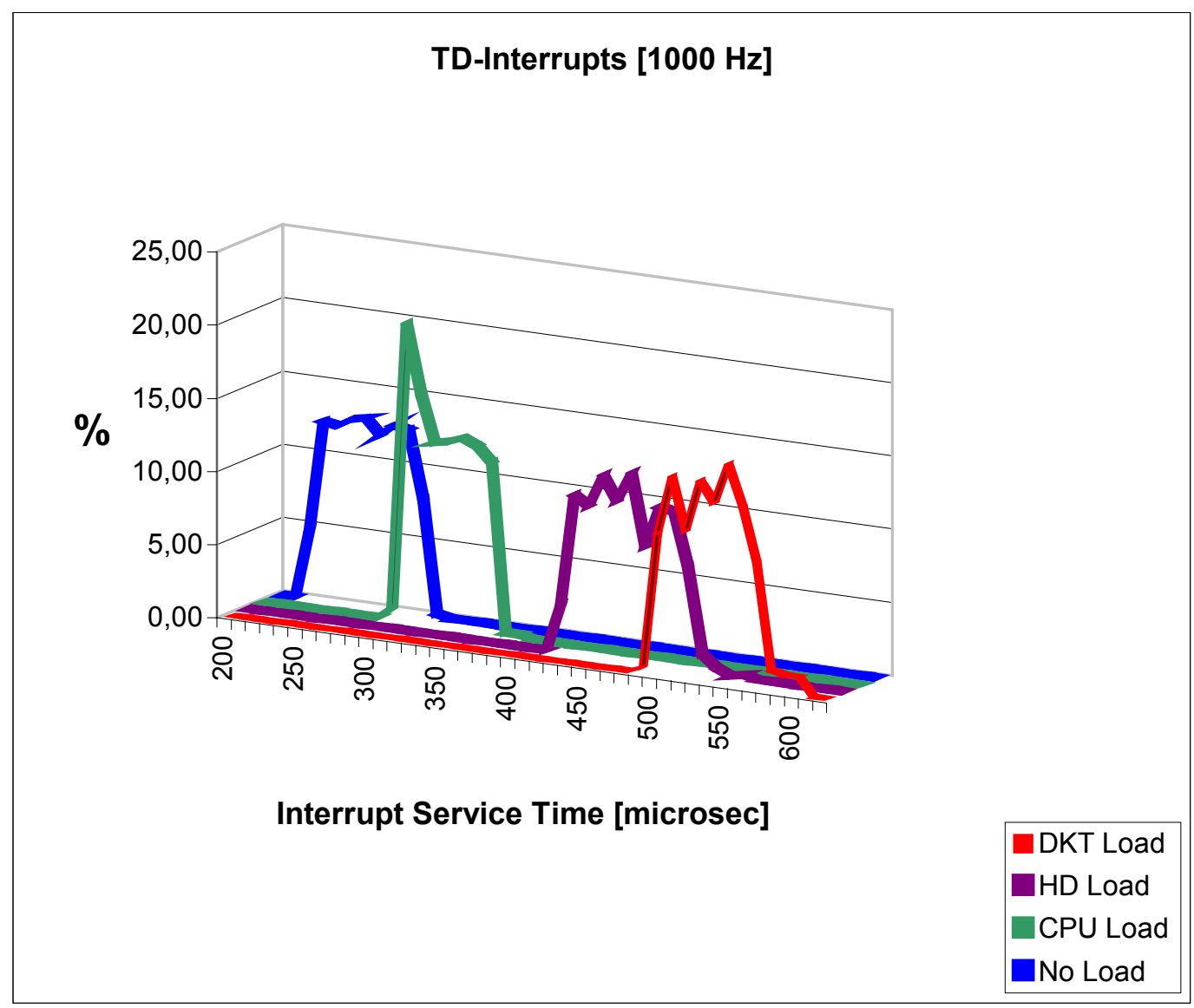

Figure C.10: Interrupt Service Time for TD-Interrupts [1000 Hz]

\section{C.2. Virtual Timer Timeliness}

A periodic RT-application correctness depends on the timeliness of its scheduling. MINIX4RT implements periodic processessing using Virtual Timers facilities. Therefore, the timeliness of Virtual Timers is an important measurament that evaluates performance of a RTOS.

These tests were carried out using the same equipment and loads as in Section C.1. 
Table C.11: Virtual Timer Timeliness for 10000 [Hz]

\begin{tabular}{|c|c|c|c|c|}
\hline Delay [ns] & DKTLoad & HDLoad & CPULoad & NOLoad \\
\hline Average & 99524 & 99524 & 99730 & 99732 \\
\hline Standard Deviation & 6773 & 7371 & 7079 & 6528 \\
\hline Minimum & 39968 & 53760 & 62880 & 60320 \\
\hline Maximum & 123712 & 142848 & 127264 & 138688 \\
\hline
\end{tabular}

Table C.12: Virtual Timer Timeliness for 5000 [Hz]

\begin{tabular}{|c|c|c|c|c|}
\hline Delay [ns] & DKTLoad & HDLoad & CPULoad & NOLoad \\
\hline Average & 199456 & 199447 & 199465 & 199464 \\
\hline Standard Deviation & 5228 & 9341 & 3335 & 1614 \\
\hline Minimum & 167328 & 154592 & 155232 & 183904 \\
\hline Maximum & 233632 & 246432 & 242880 & 213536 \\
\hline
\end{tabular}

Table C.13: Virtual Timer Timeliness for 1000 [Hz]

\begin{tabular}{|c|c|c|c|c|}
\hline Delay [ns] & DKTLoad & HDLoad & CPULoad & NOLoad \\
\hline Average & 999833 & 999772 & 999839 & 999799 \\
\hline Standard Deviation & 7277 & 12511 & 3625 & 3896 \\
\hline Minimum & 955424 & 936064 & 961024 & 975520 \\
\hline Maximum & 1048064 & 1041728 & 1038496 & 1023712 \\
\hline
\end{tabular}

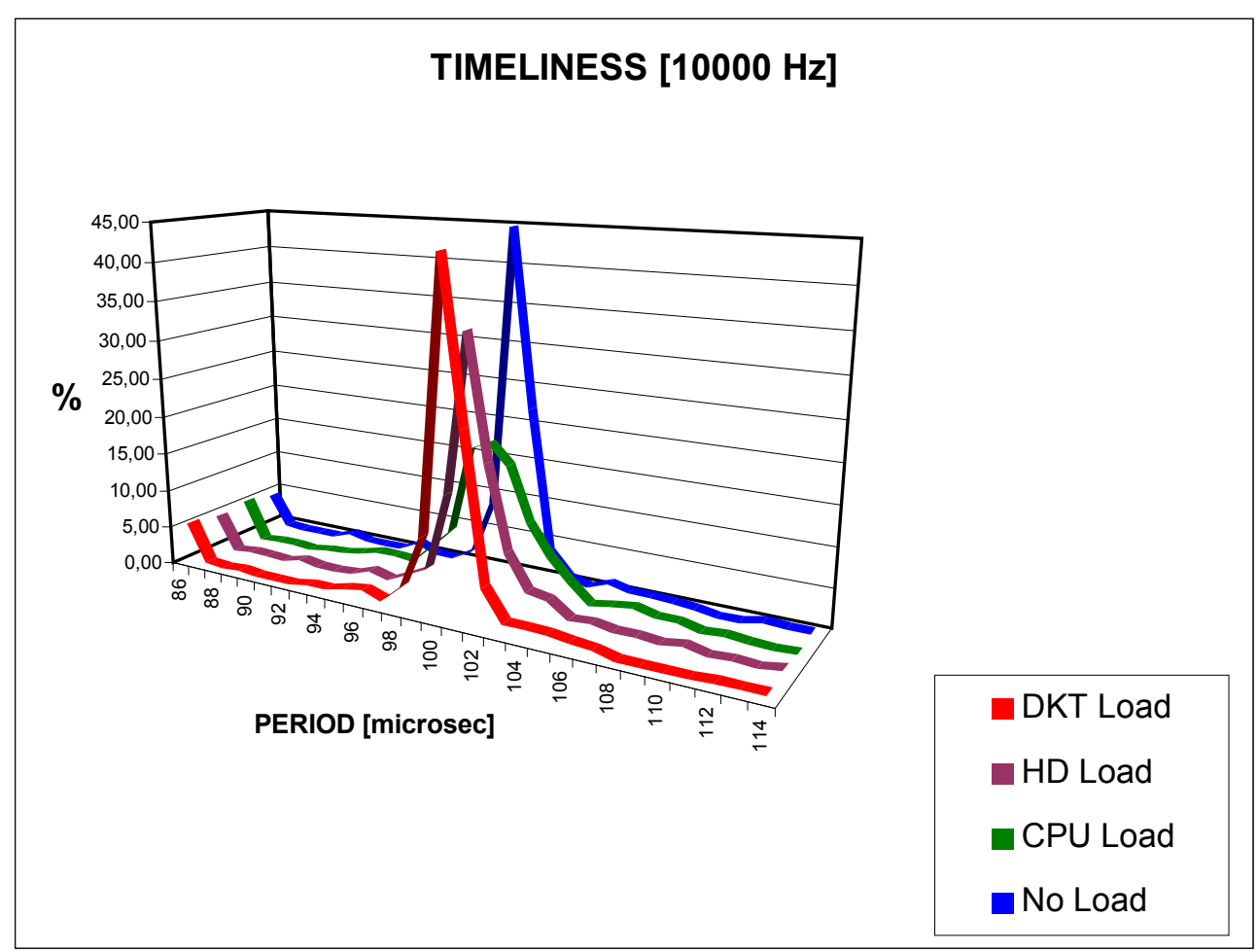

Figure C.11: Virtual Timer Timeliness for 10000 [Hz] 


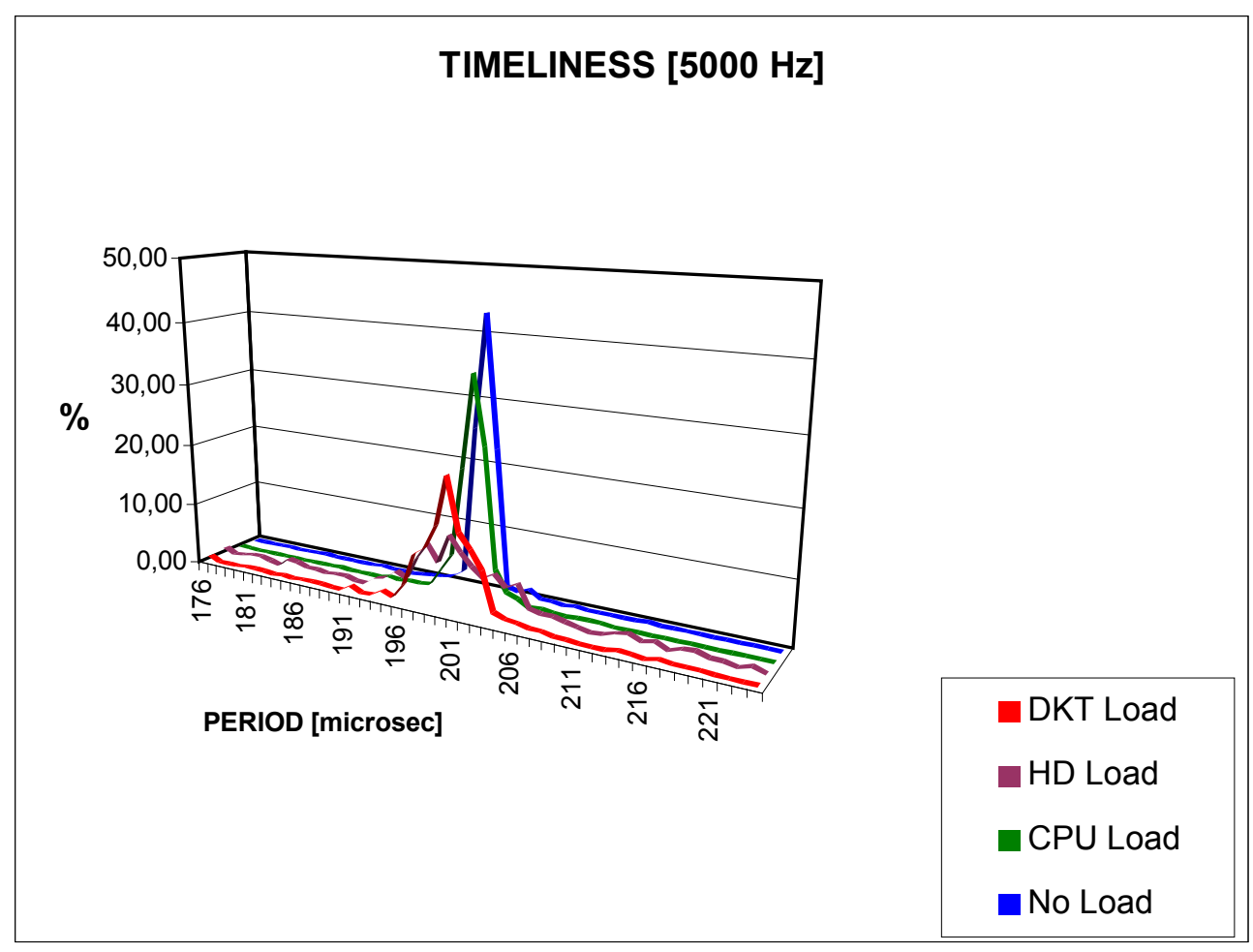

Figure C.12: Virtual Timer Timeliness for 5000 [Hz]

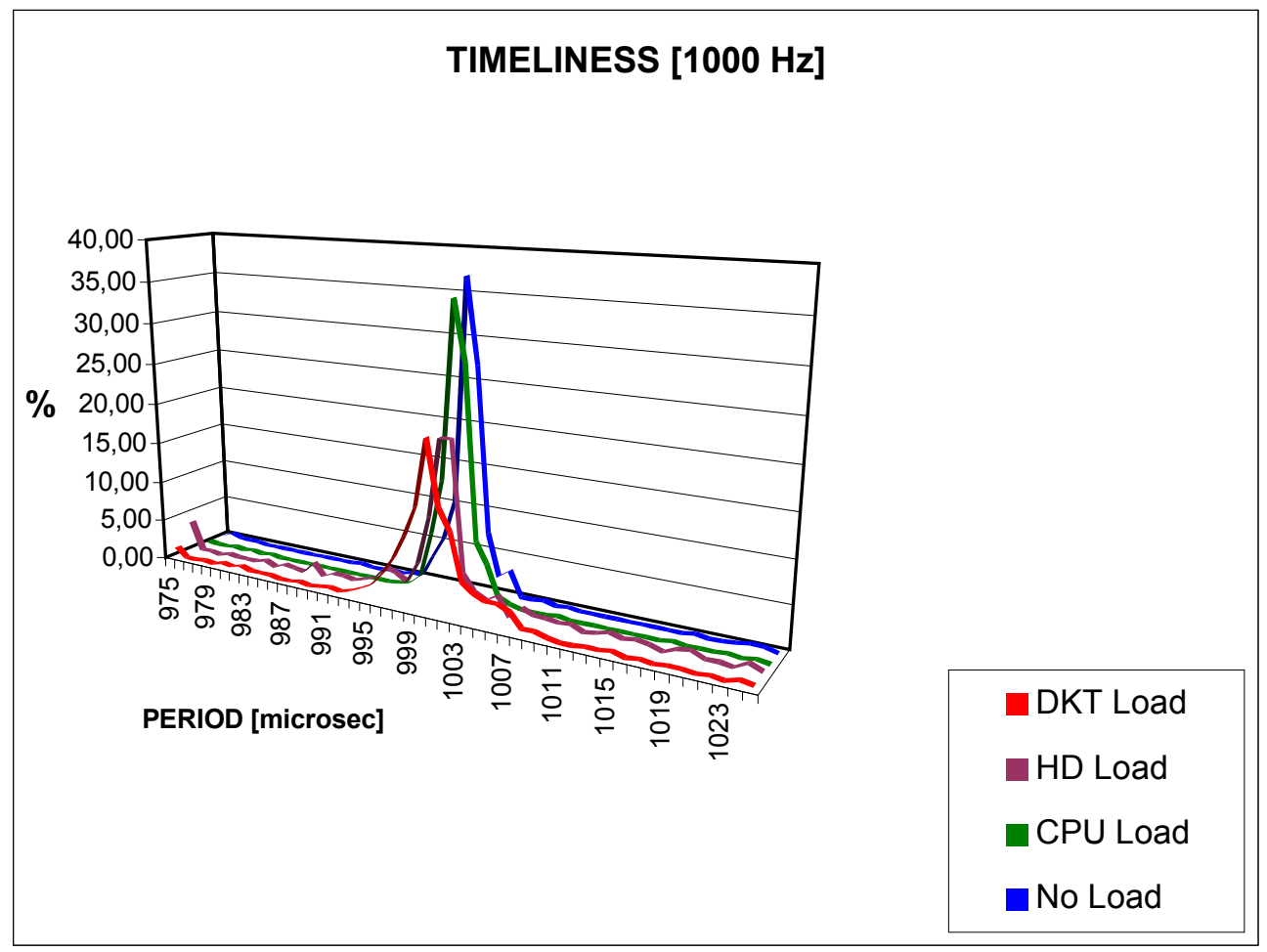

Figure C.13: Virtual Timer Timeliness for $1000[\mathrm{~Hz}]$ 


\section{C.3. RT-IPC performance}

The equipment used for the tests was:

- PAP: IBM Model 370C Notebook, Intel® DX4 75MHz on system board, AT Bus, Memory $8 \mathrm{MB}$, Diskette Drive 1.44MB, Hard Disk Drive 540MB 2.5-inch, PCMCIA One Type-III or two Type-II. Software: MINIX4RT (Kernel 20122005).

As in MINIX4RT messages can be transfered in two ways (synchronous or asynchronous) and with or without priority inheritance (BPIP), four sets of tests were carried out to evaluate the performance of each.

\section{C.3.1. Synchronous Message Transfers Tests without BPIP and without}

\section{Timeouts}

Table C.14 shows the test results. Listing C.1 shows pseudocode of two programs:

- FATHER: Makes a loop sending request messages to SON and receiving the replies.

- SON: Makes a loop receiving request messages and sending replies.

Listing C.1: Pseudocode of Programs to Test Synchronous Message Transfers without BPIP and without Timeouts

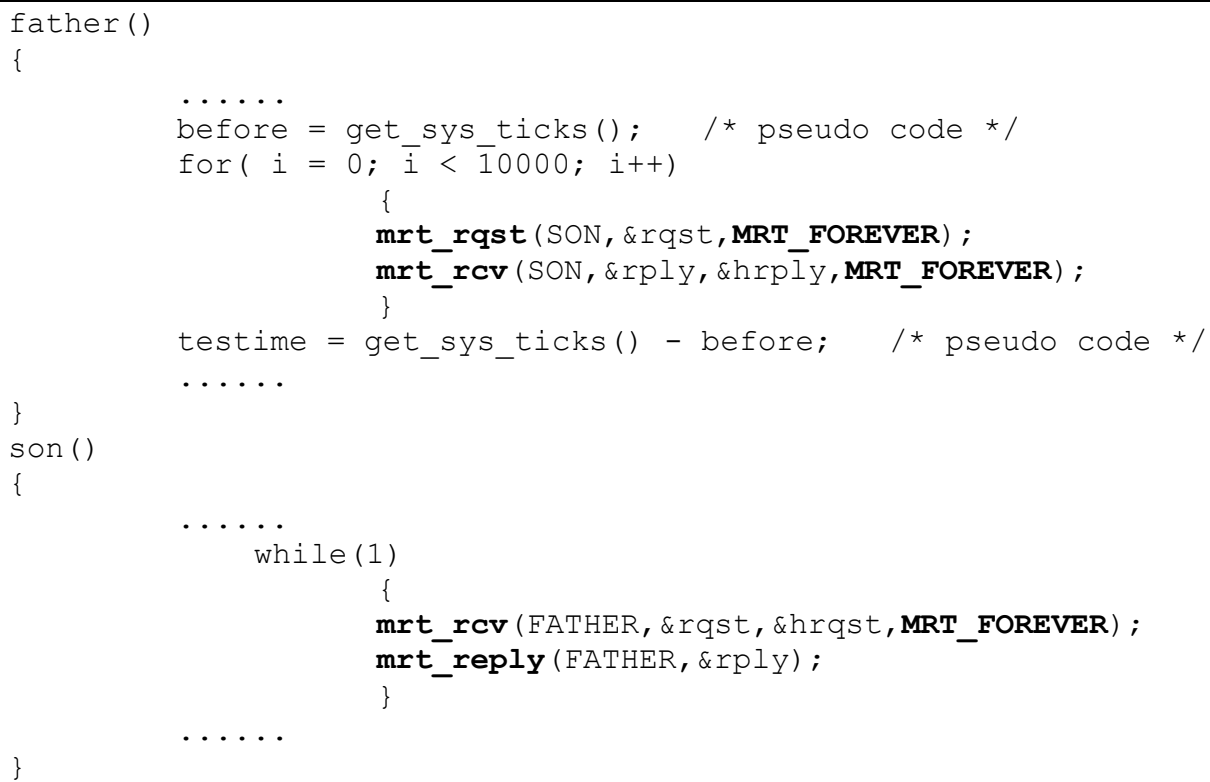


Table C.14: Synchronous Message Transfers Test Results without BPIP and without Timeouts

\begin{tabular}{|c|c|c|}
\hline Clock Freq & 1000 & ticks/s \\
\hline Testing time & 1925 & ticks \\
\hline mrt_arqst & 10000 & $\mathrm{msgs}$ \\
\hline mrt_reply & 10000 & $\mathrm{msgs}$ \\
\hline Total msg & 20000 & $\mathrm{msgs}$ \\
\hline Throughput & 10390 & $\mathrm{msgs} / \mathrm{s}$ \\
\hline RT-schedulings & 20000 & scheds \\
\hline Time by msg & 96 & microsec/msg \\
\hline
\end{tabular}

\section{C.3.2. Asynchronous Message Transfers Tests without BPIP and without}

\section{Timeouts}

Table C. 15 shows the test results. Listing C. 2 shows pseudocode of two processes:

- FATHER: makes two loops. An internal loop sending 10 asynchronous request messages to $\mathrm{SON}$. An an external loop receiving replies.

- SON: makes two loops. An internal loop receiving 10 messages from

FATHER. An an external loop sending replies.

Listing C.2: Pseudocode of Programs to Test Asynchronous Message Transfers without BPIP and without Timeouts

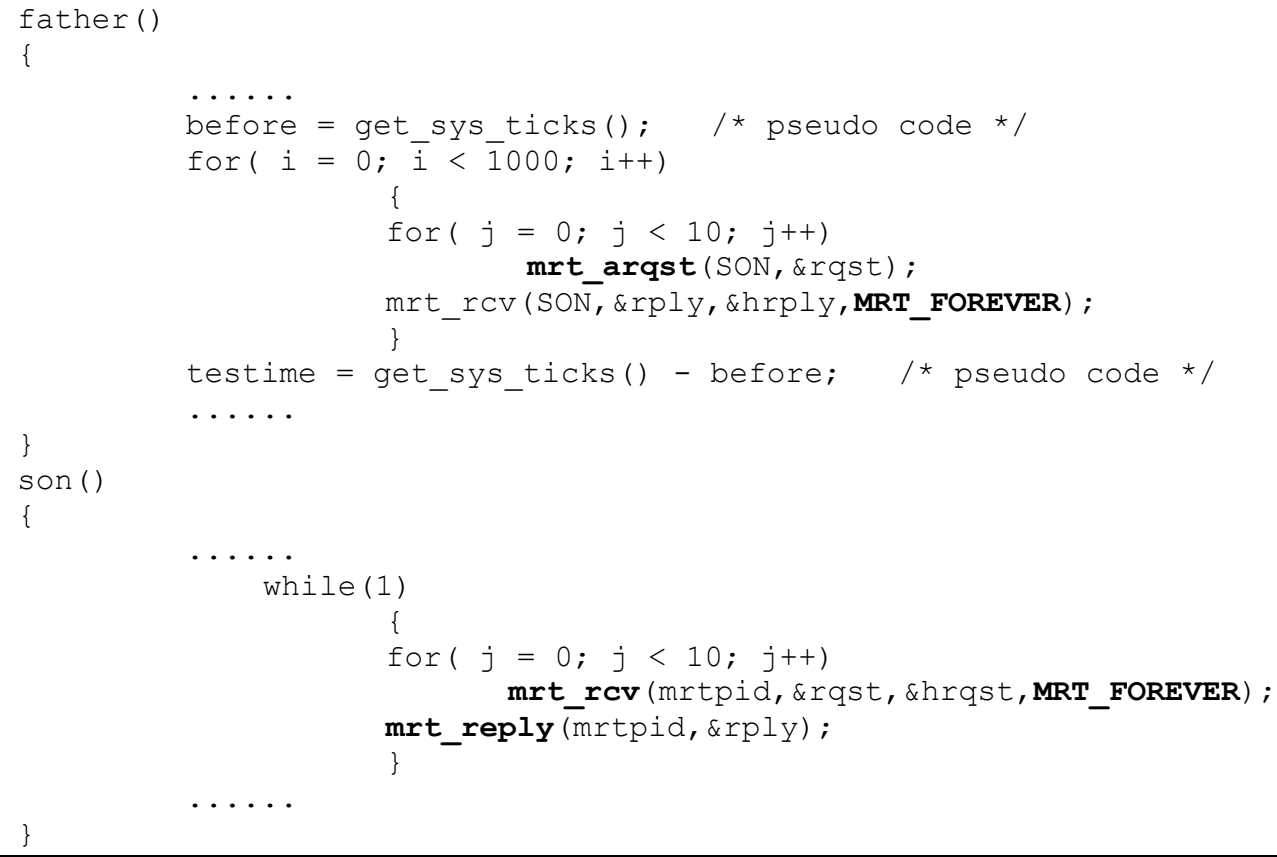


Table C.15: Asynchronous Message Transfers Test Results without BPIP and without Timeouts

\begin{tabular}{|c|c|c|}
\hline Clock Freq & 1000 & ticks/s \\
\hline Testing time & 993 & ticks \\
\hline mrt_arqst & 10000 & $\mathrm{msgs}$ \\
\hline mrt_reply & 1000 & $\mathrm{msgs}$ \\
\hline Total msg & 11000 & $\mathrm{msgs}$ \\
\hline Throughput & 11078 & $\mathrm{msgs} / \mathrm{s}$ \\
\hline RT-schedulings & 4000 & scheds \\
\hline Time by msg & 90 & microsec $/ \mathrm{msg}$ \\
\hline
\end{tabular}

\section{C.3.3. Synchronous Message Transfers Tests with BPIP and without}

Timeouts

This test was carried out using the programs of C.3.1. Table C.16 shows the test results.

Table C.16: Synchronous Message Transfers Test Results with BPIP and without Timeouts

\begin{tabular}{|c|c|c|}
\hline Clock Freq & 1000 & ticks/s \\
\hline Testing time & 2164 & ticks \\
\hline mrt_arqst & 10000 & $\mathrm{msgs}$ \\
\hline mrt_reply & 10000 & $\mathrm{msgs}$ \\
\hline Total msg & 20000 & $\mathrm{msgs}$ \\
\hline Throughput & 9242 & $\mathrm{msgs} / \mathrm{s}$ \\
\hline RT-schedulings & 20000 & scheds \\
\hline Time by msg & 108 & microsec/msg \\
\hline
\end{tabular}

\section{C.3.4. Asynchronous Message Transfers Tests with BBIP and without}

Timeouts

This test was carried out using the programs of C.3.2. Table C.17 shows the test results.

Table C.17: Asynchronous Message Transfers Test Results with BPIP and without Timeouts

\begin{tabular}{|c|c|c|}
\hline Clock Freq & 1000 & ticks/s \\
\hline Testing time & 1008 & ticks \\
\hline mrt_arqst & 10000 & $\mathrm{msgs}$ \\
\hline mrt_reply & 1000 & $\mathrm{msgs}$ \\
\hline Total msg & 11000 & $\mathrm{msgs}$ \\
\hline Throughput & 10912 & $\mathrm{msgs} / \mathrm{s}$ \\
\hline RT-schedulings & 4000 & scheds \\
\hline Time by msg & 91 & microsec/msg \\
\hline
\end{tabular}

\section{C.3.5. Request/Receive Tests with BPIP and without Timeouts}

Table C.18 shows the test results. Listing C.3 shows pseudocode of two programs: 
- FATHER: Makes a loop sending request messages to SON and receiving the replies using the mrt_rqrcv() kernel call.

- SON: Makes a loop receiving request messages and sending replies.

Listing C.3: Pseudocode of Programs to Test Request/Receive with BPIP and without Timeouts

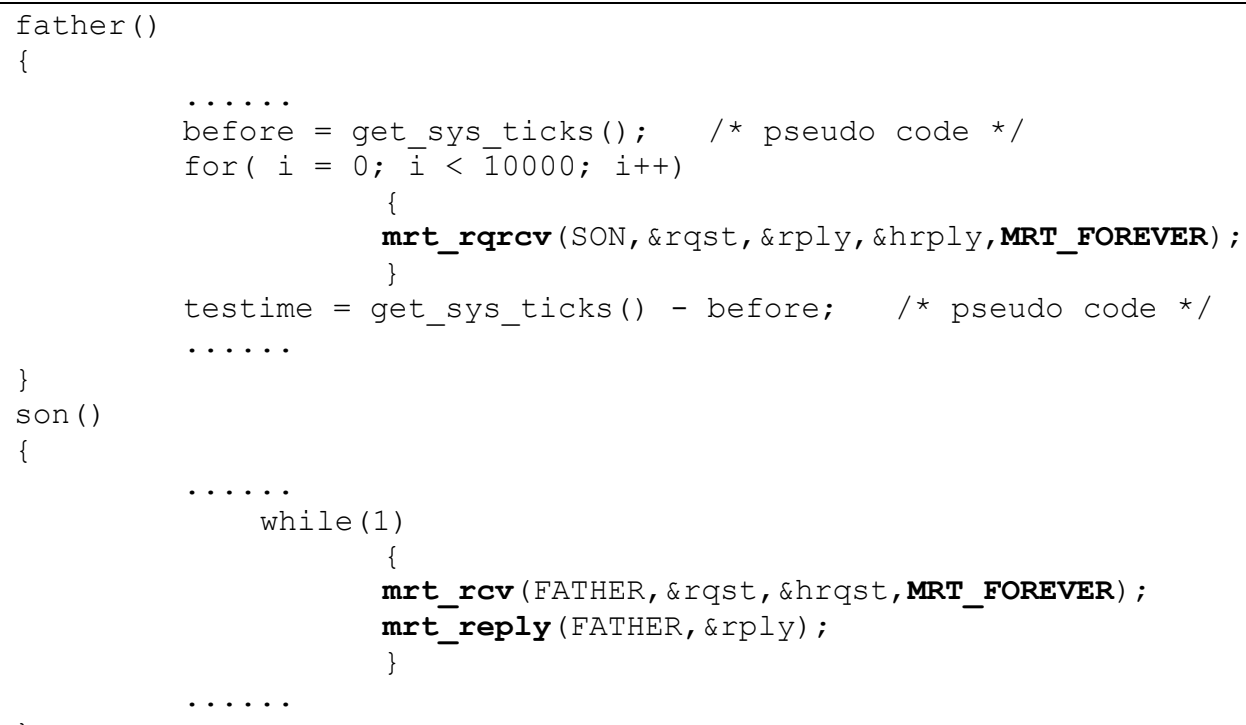

Table C.18 Request/Receive Test Results with BPIP and without Timeouts

\begin{tabular}{|c|c|c|}
\hline Clock Freq & 1000 & ticks/s \\
\hline Testing time & 1749 & ticks \\
\hline mrt_rqrev & 10000 & $\mathrm{msgs}$ \\
\hline mrt_reply & 10000 & $\mathrm{msgs}$ \\
\hline Total msg & 20000 & $\mathrm{msgs}$ \\
\hline Throughput & 11435 & $\mathrm{msgs} / \mathrm{s}$ \\
\hline RT-schedulings & 20000 & scheds \\
\hline Time by msg & 87 & microsec $/ \mathrm{msg}$ \\
\hline
\end{tabular}

\section{C.3.6. Synchronous Message Transfers Tests with BPIP and with Timeouts}

Table C.19 shows the test results. Listing C.4shows pseudocode of two processes:

Listing C.4: Pseudocode of Programs to Test Synchronous Message Transfers with BPIP and with Timeouts

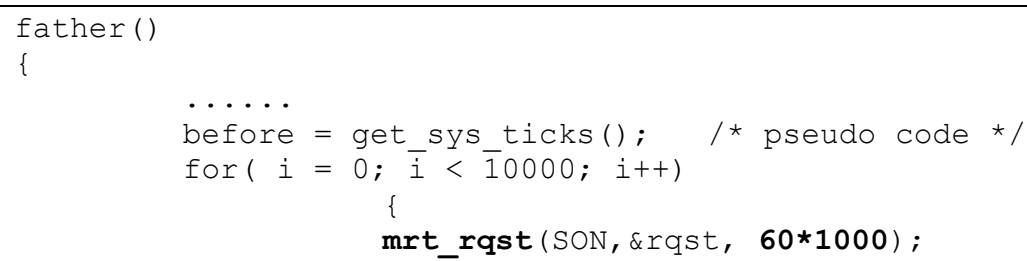




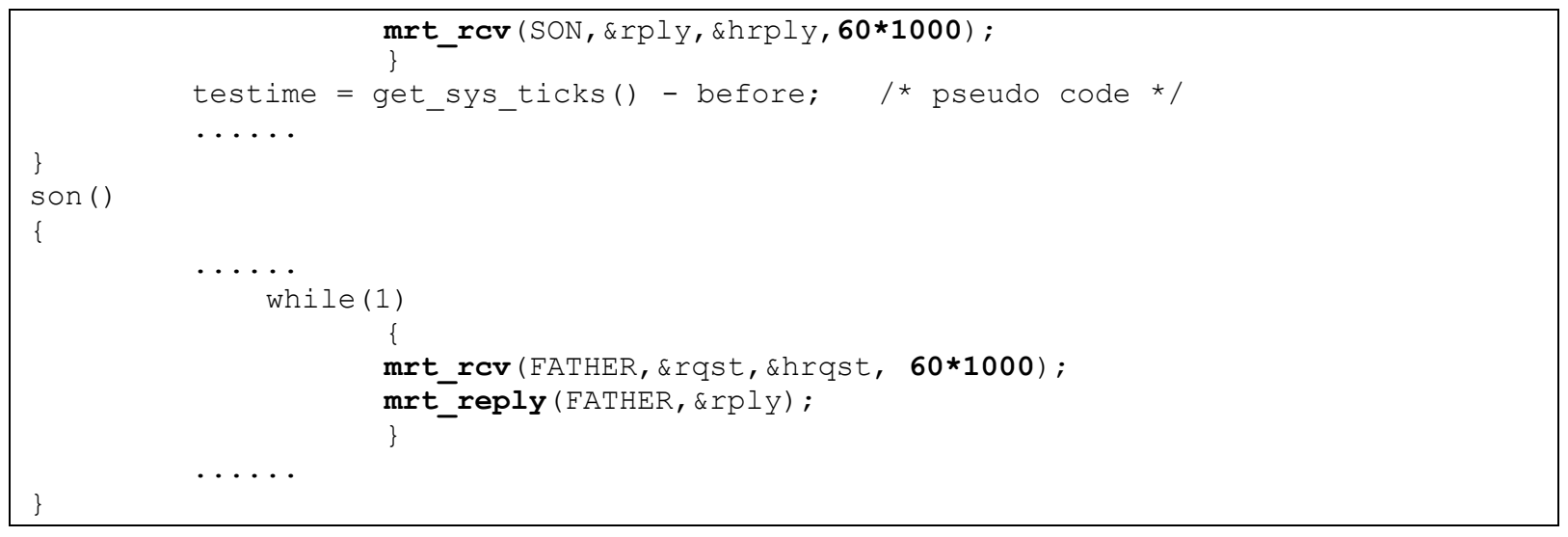

Table C.19: Synchronous Message Transfers Test Results with BPIP and with Timeouts

\begin{tabular}{|c|c|c|}
\hline Clock Freq & 1000 & ticks/s \\
\hline Testing time & 2222 & ticks \\
\hline mrt_arqst & 10000 & $\mathrm{msgs}$ \\
\hline mrt_reply & 10000 & $\mathrm{msgs}$ \\
\hline Total msg & 20000 & $\mathrm{msgs}$ \\
\hline Throughput & 9000 & $\mathrm{msgs} / \mathrm{s}$ \\
\hline RT-schedulings & 20000 & scheds \\
\hline Time by msg & 111 & microsec $/ \mathrm{msg}$ \\
\hline
\end{tabular}

\section{C.3.7. Asynchronous Message Transfers Tests with BBIP and with Timouts}

Table C.20 shows the test results. Listing C.5 shows pseudocode of two processes:

Listing C.5: Pseudocode of Programs to Test Asynchronous Message Transfers with BPIP and with Timeouts

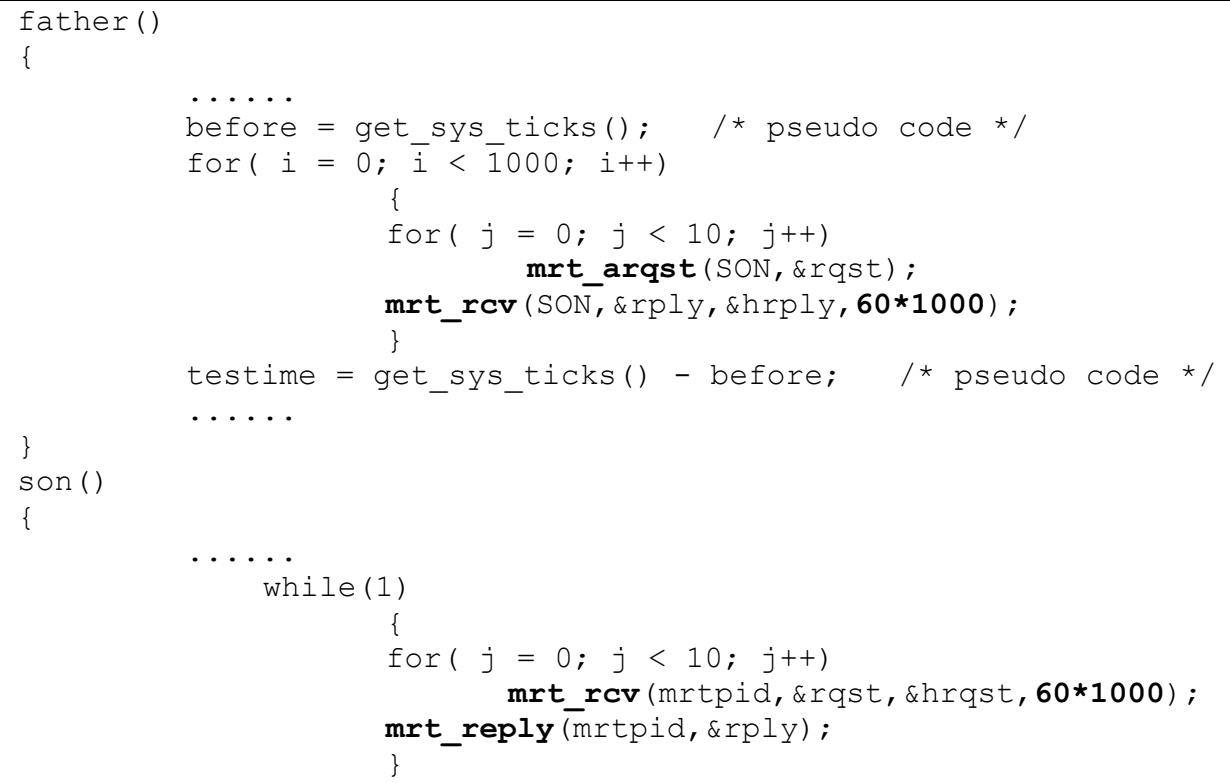


Table C.20: Asynchronous Message Transfers Test Results with BPIP and without Timouts

\begin{tabular}{|c|c|c|}
\hline Clock Freq & 1000 & ticks/s \\
\hline Testing time & 1071 & ticks \\
\hline mrt_arqst & 10000 & $\mathrm{msgs}$ \\
\hline mrt_reply & 1000 & $\mathrm{msgs}$ \\
\hline Total msg & 11000 & $\mathrm{msgs}$ \\
\hline Throughput & 10270 & $\mathrm{msgs} / \mathrm{s}$ \\
\hline RT-schedulings & 4000 & scheds \\
\hline Time by msg & 97 & microsec $/ \mathrm{msg}$ \\
\hline
\end{tabular}

\section{C.3.8. Test Request/Receive Tests with BPIP and with Timeouts}

Table C. 21 shows the test results. Listing C.6 shows pseudocode of two processes:

Listing C.6: Pseudocode of Programs to Test Synchronous Message Transfers with BPIP and with Timeouts

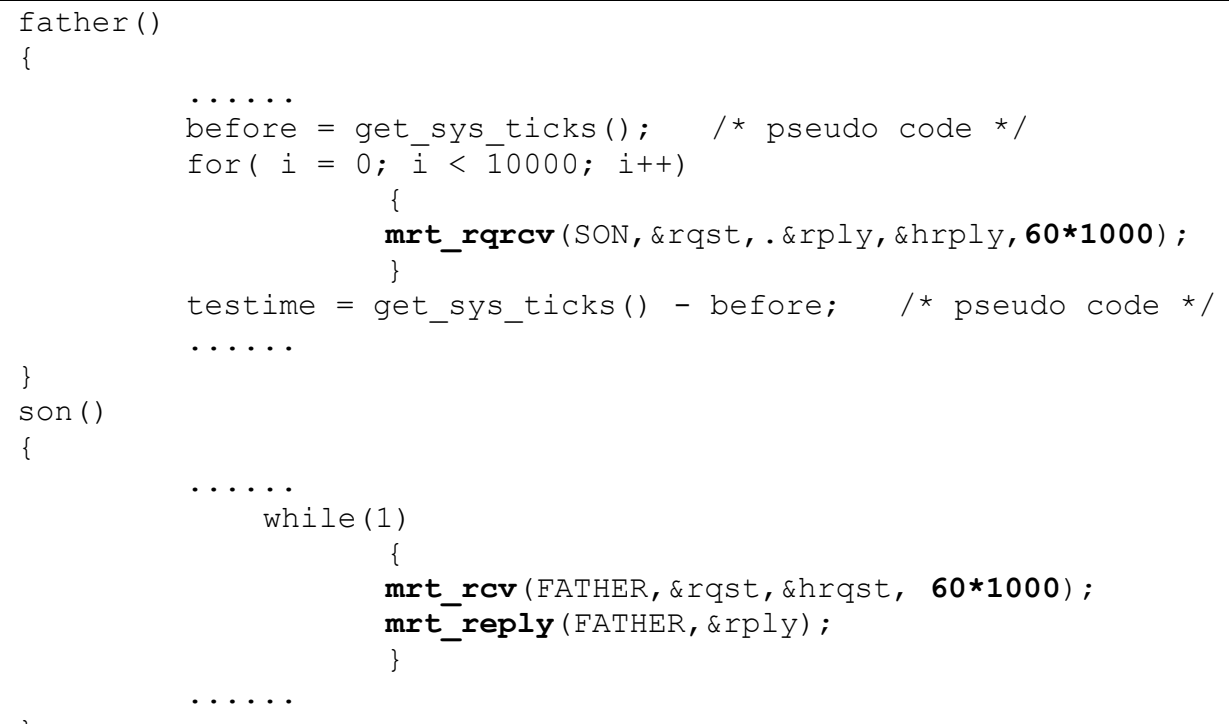

Table C.21: Synchronous Message Transfers Test Results with BPIP and with Timeouts

\begin{tabular}{|c|c|c|}
\hline Clock Freq & 1000 & ticks/s \\
\hline Testing time & 1754 & ticks \\
\hline mrt_rqrev & 10000 & $\mathrm{msgs}$ \\
\hline mrt_reply & 10000 & $\mathrm{msgs}$ \\
\hline Total msg & 20000 & $\mathrm{msgs}$ \\
\hline Throughput & 11402 & $\mathrm{msgs} / \mathrm{s}$ \\
\hline RT-schedulings & 20000 & scheds \\
\hline Time by msg & 87 & microsec $/ \mathrm{msg}$ \\
\hline
\end{tabular}


C.3.9. RT-IPC Tests Results

Figure C.11 shows a comparison of Message Time for all tests.

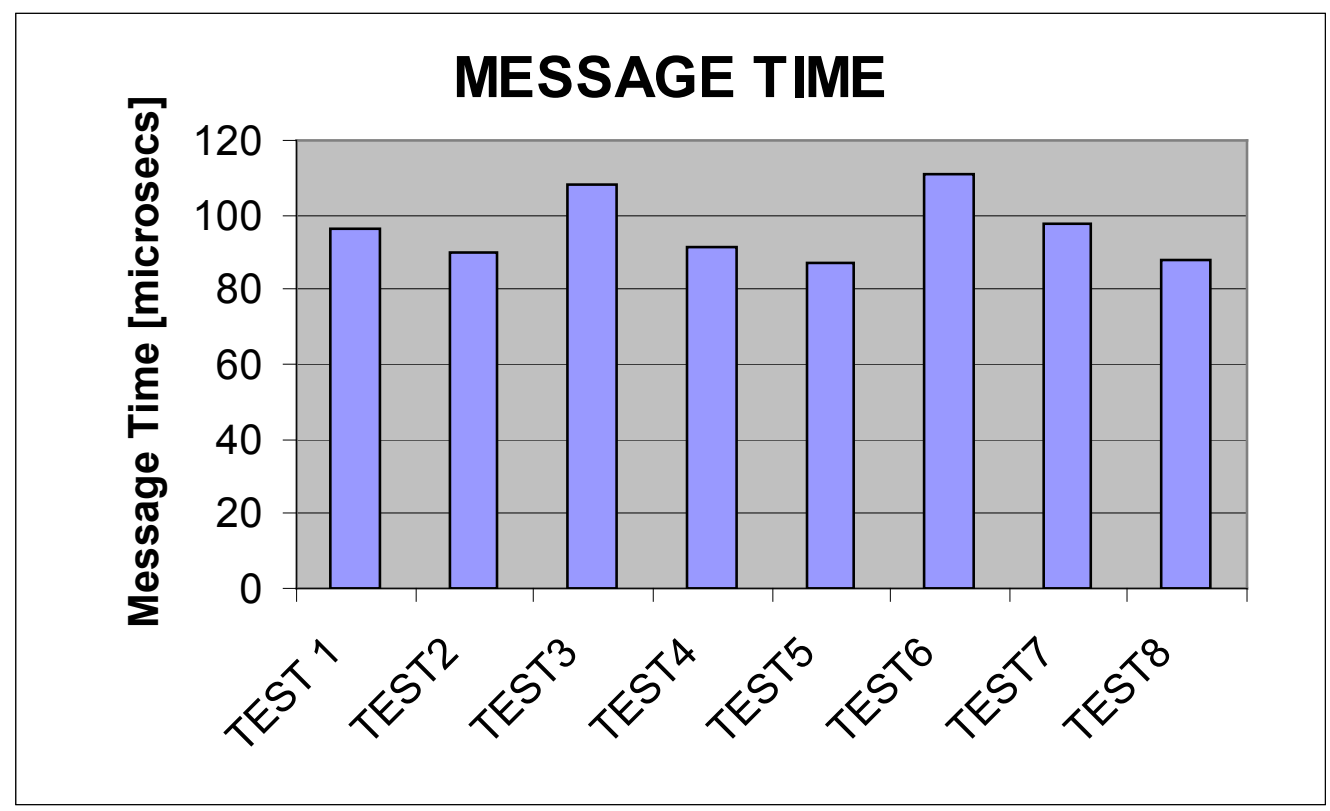

Figure C.11: Virtual Timer Timeliness for 10000 [Hz]

Figure C.12 shows a comparison of Message Thoughput for all tests.

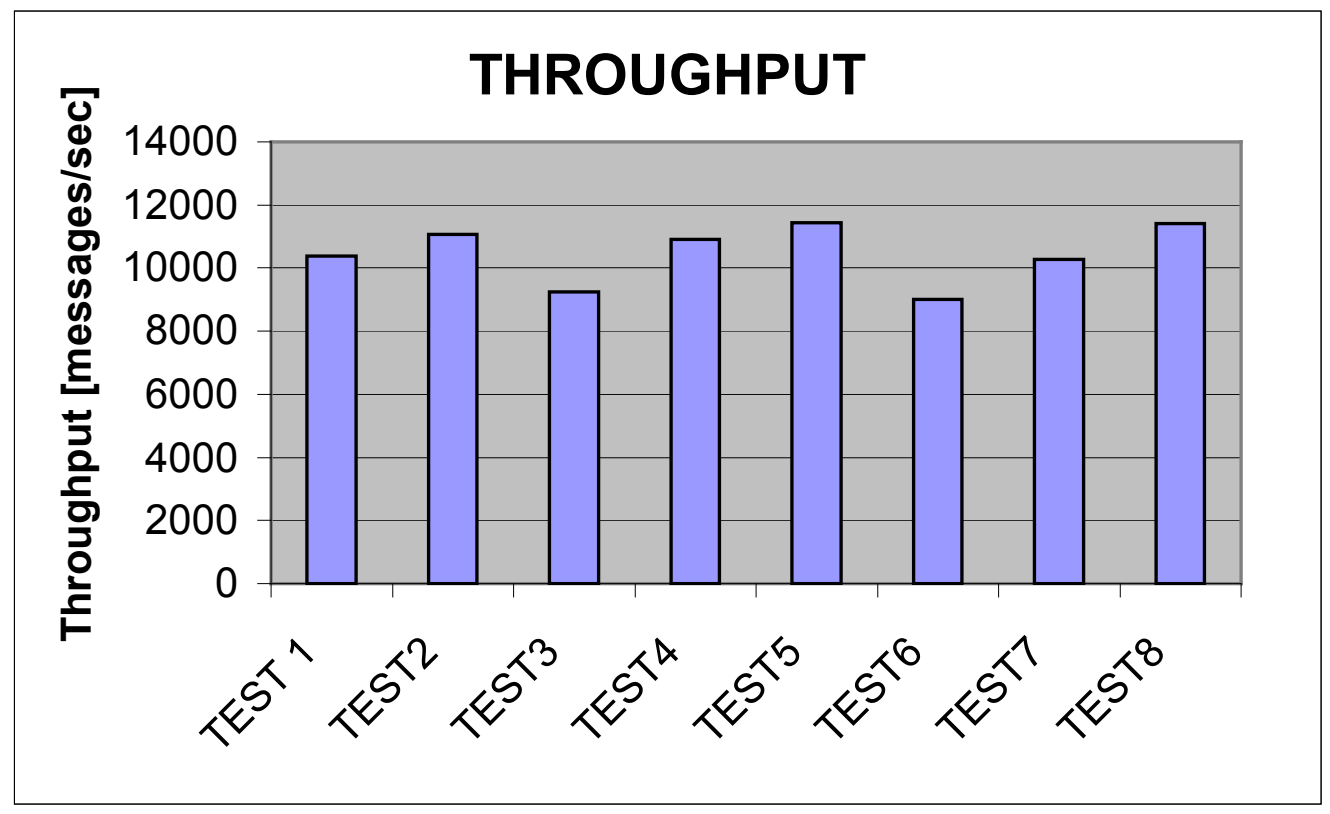

Figure C.12: Virtual Timer Timeliness for $10000[\mathrm{~Hz}]$ 


\section{Appendix D: SYSTEM DATA STRUCTURES}

\section{D.1. User-Level Data Structures}

The following data structures are used by User-Level programs.

\section{D.1.1. System-wide Data Structures}

Listing D.1: System-wide Data Structures.

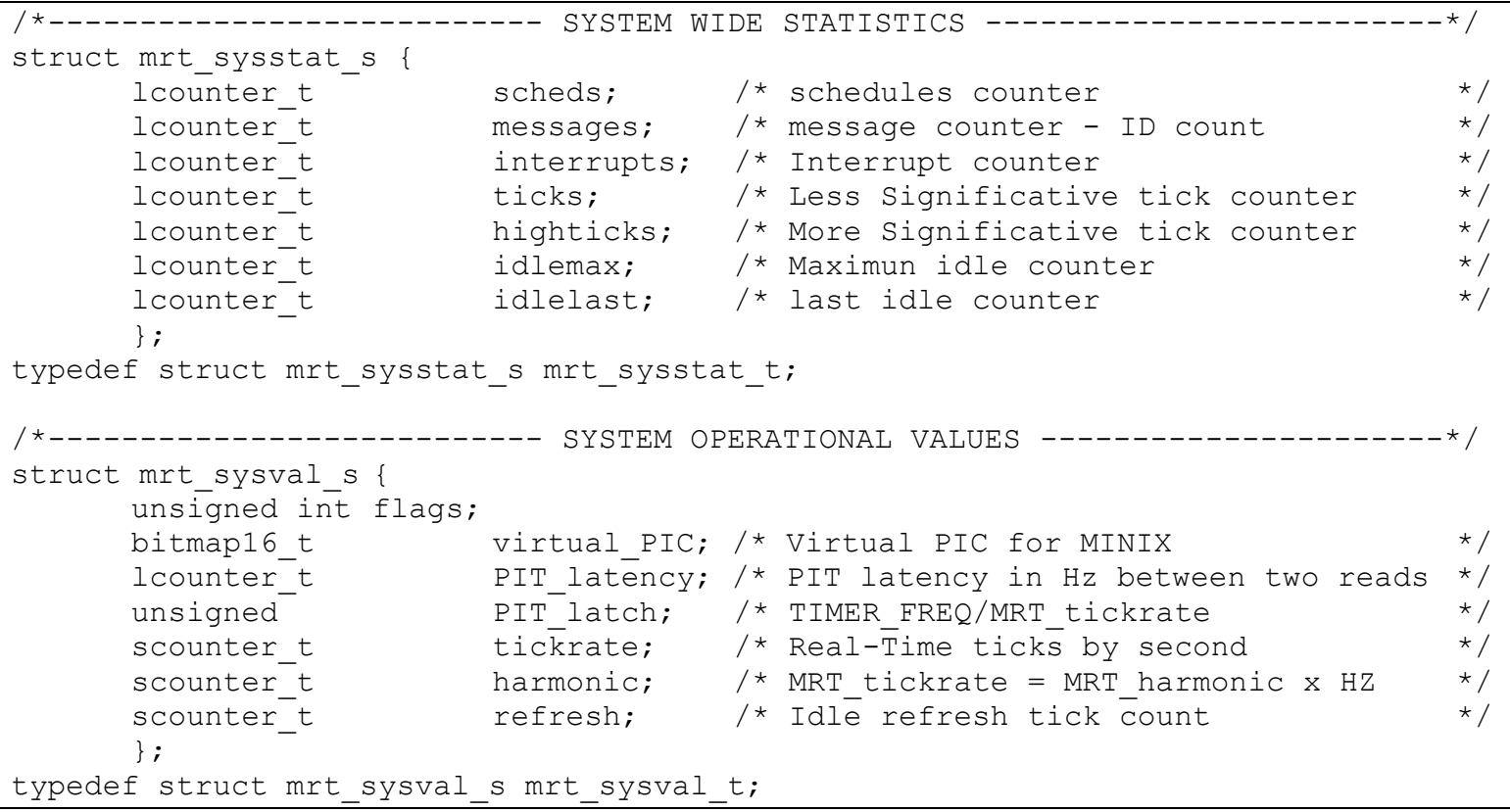

f struct mrt_sysval_s mrt_sysval_t;

\section{D.1.2. Interrupt-related Data Structures}

Listing D.2: Interrupt-related Data Structures.

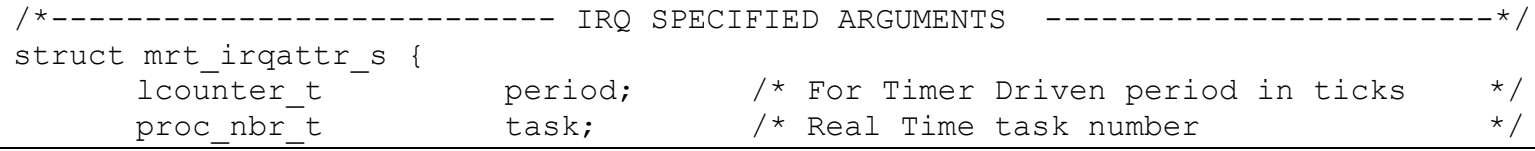




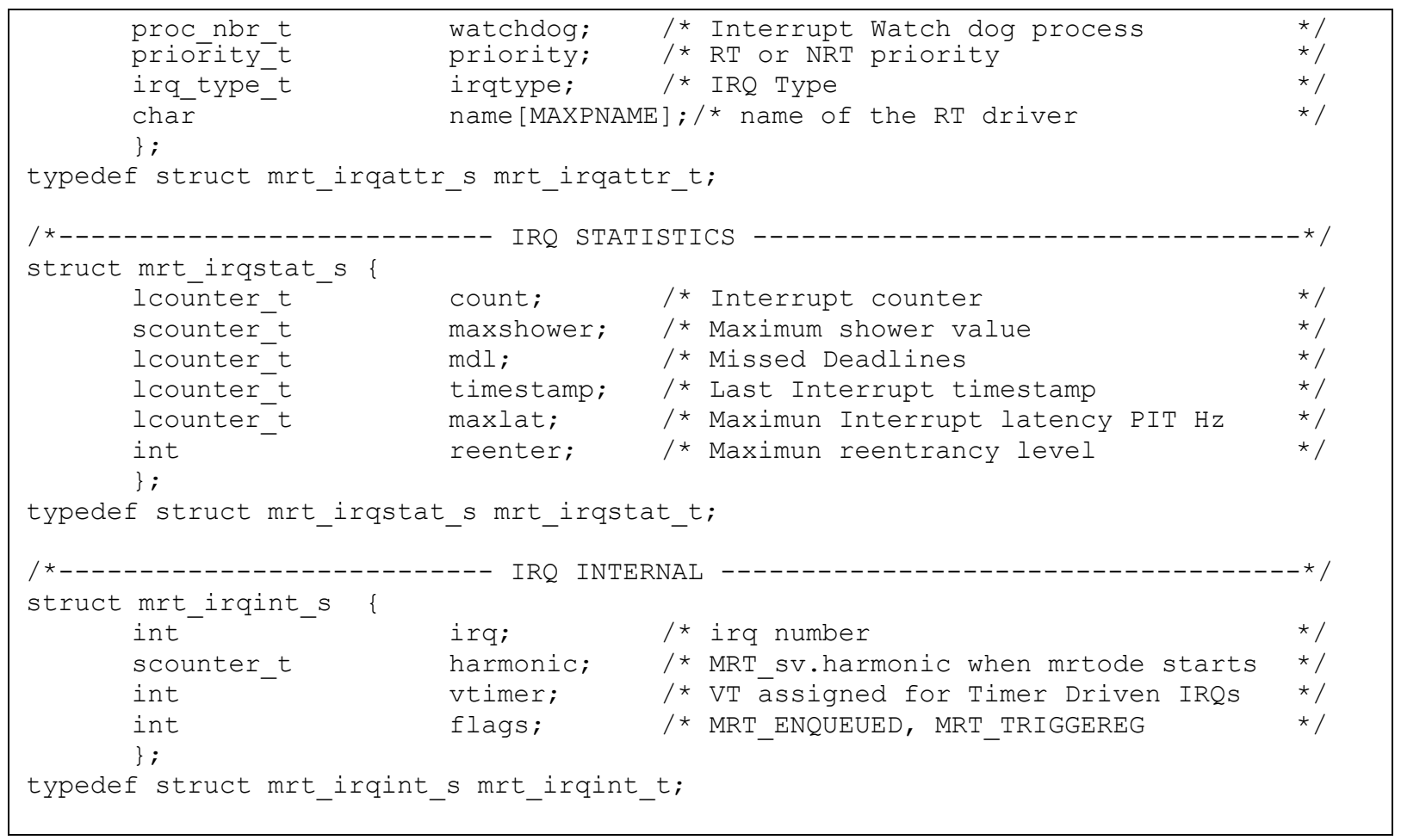

\section{D.1.3. Process-related Data Structures}

\section{Listing D.3: Process-related Data Structures.}

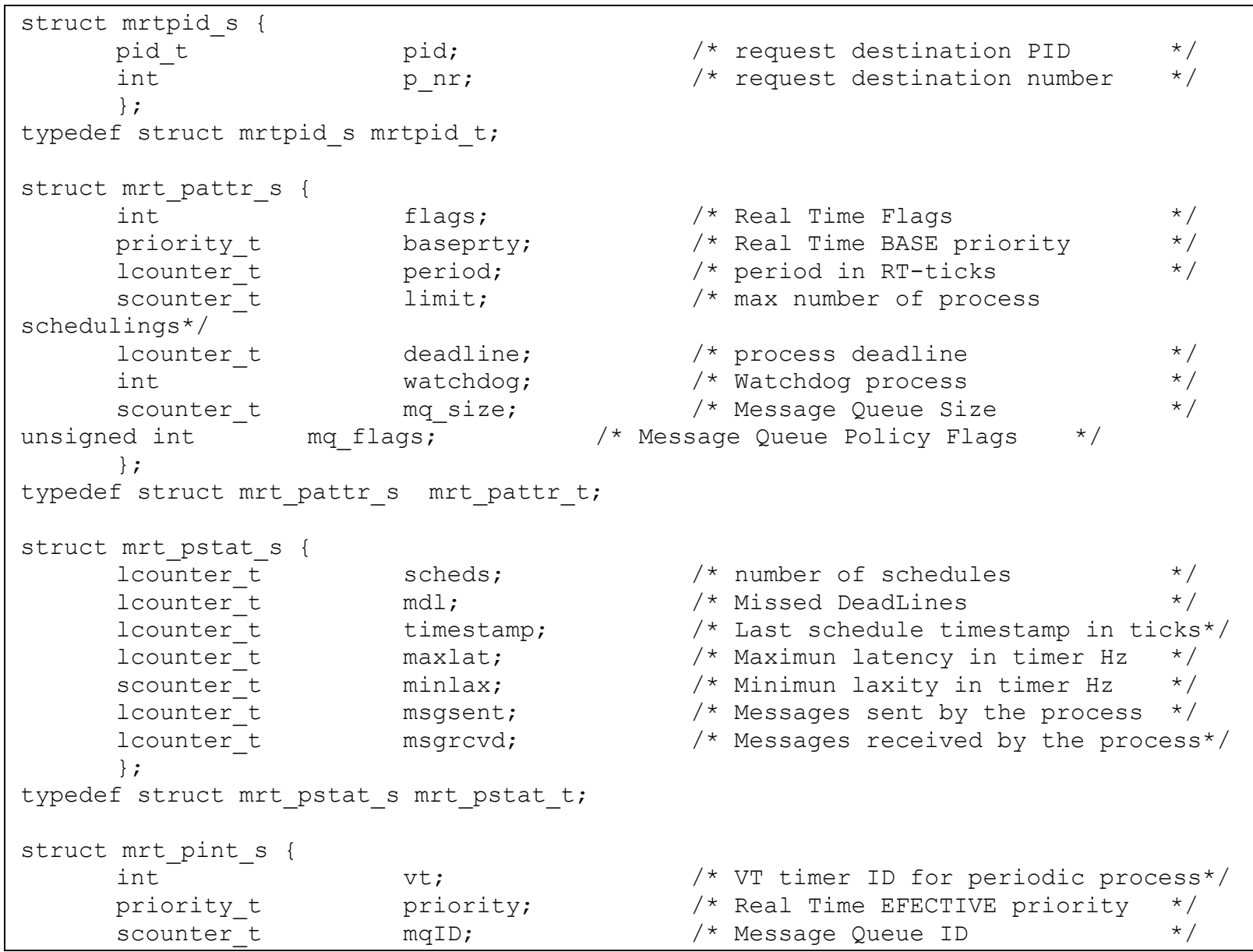




\begin{tabular}{ccc}
\hline int & p_nr; \\
typedef struct mrt_pint_s mrt_pint_t; & /* process slot
\end{tabular}

\section{D.1.4. Kernel Calls-related Data Structures}

Listing D.4: Kernel Calls-related Data Structures.

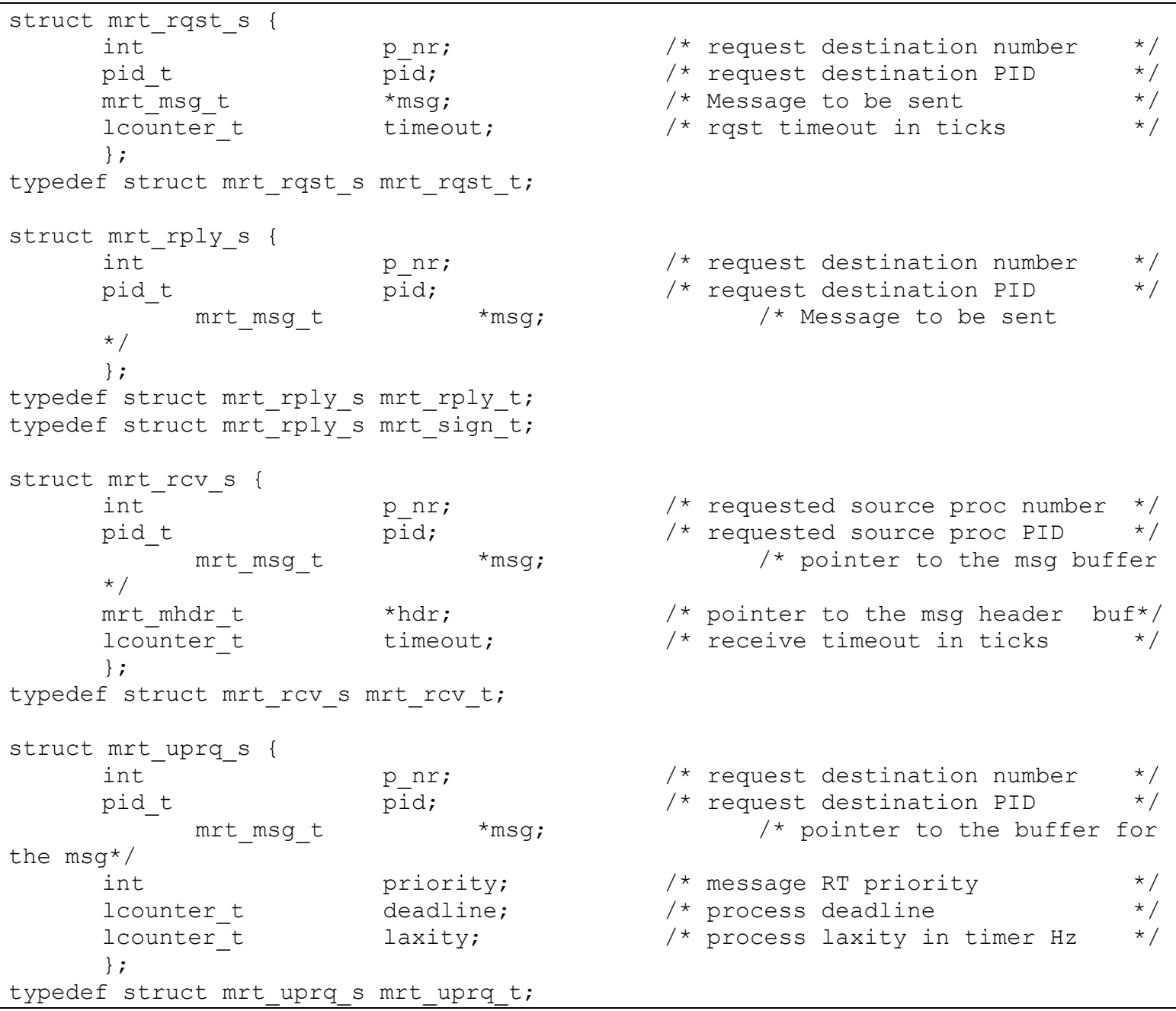

\section{D.1.5. Message-related Data Structures}

Listing D.5: Message-related Data Structures.

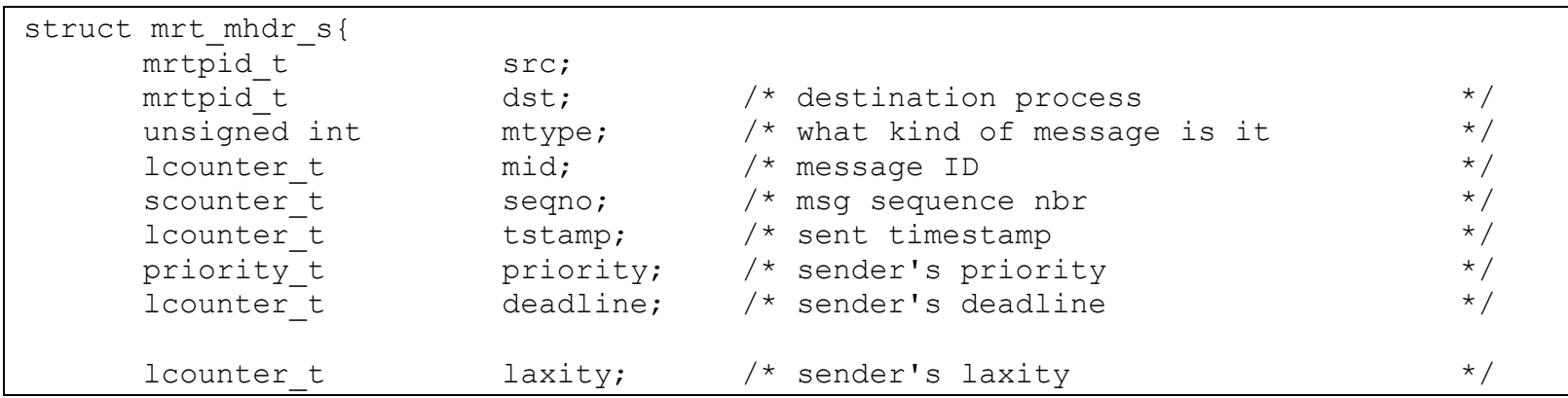




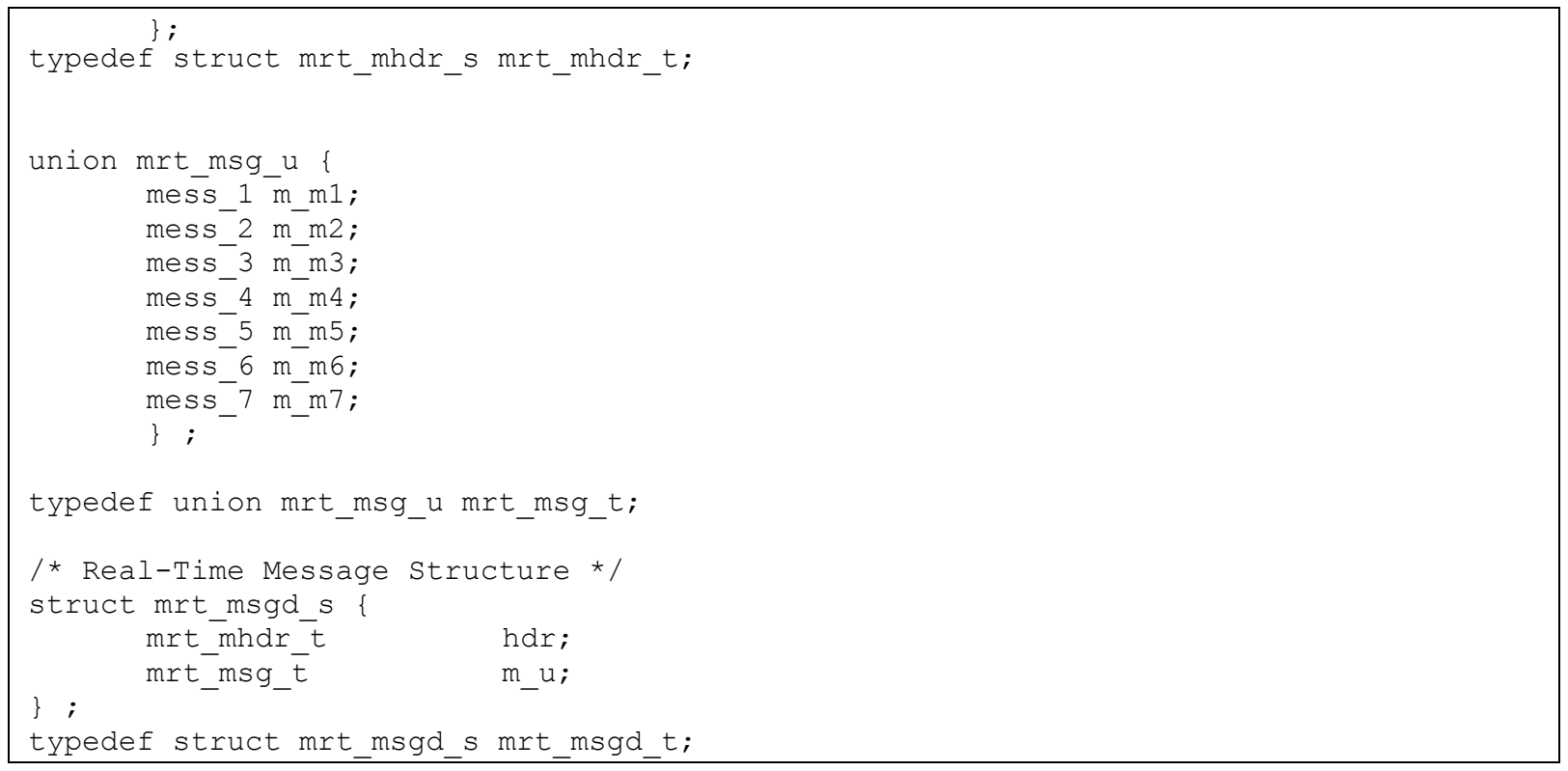

\section{D.2. Kernel-Level Data Structures}

The following data structures are used by Kernel-Level programs.

\section{D.2.1. System-wide Data Structures}

Listing D.6: System-wide Data Structures.

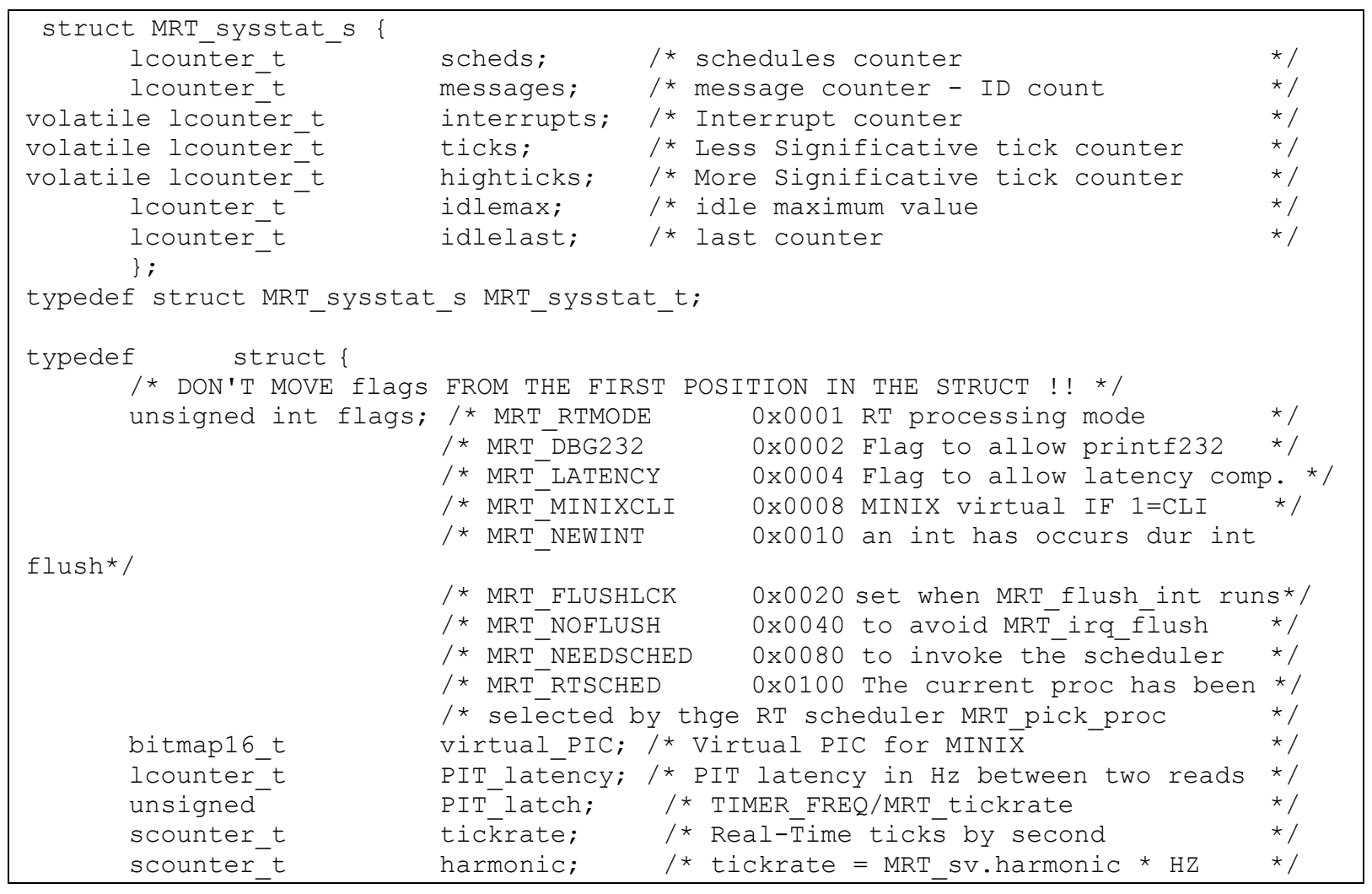




\begin{tabular}{|c|c|c|c|}
\hline $\begin{array}{l}\text { scounter_t } \\
\text { int }\end{array}$ & $\begin{array}{l}\text { refresh; } \\
\text { MINIX_soft; }\end{array}$ & $\begin{array}{l}\text { /* ticks to refresh idlerfsh } \\
\text { /* MINIX CLOCK Software interrupt }\end{array}$ & $\begin{array}{l}* / \\
* /\end{array}$ \\
\hline priority_t & prtylvl; & I* Current syetem priority level & */ \\
\hline lcounter-t & idlecount; & /* idle loop counter & $\star /$ \\
\hline scounter $t$ & idlerfsh; & I* idle refresh loop counter & */ \\
\hline $\begin{array}{l}\text { MRT_sysstat } t \\
\} \text { MRT_sysval_t; }\end{array}$ & counter; & I* system statistics & */ \\
\hline
\end{tabular}

\section{D.2.2. Interrupt-related Data Structures}

Listing D.7: Interrupt-related Data Structures.

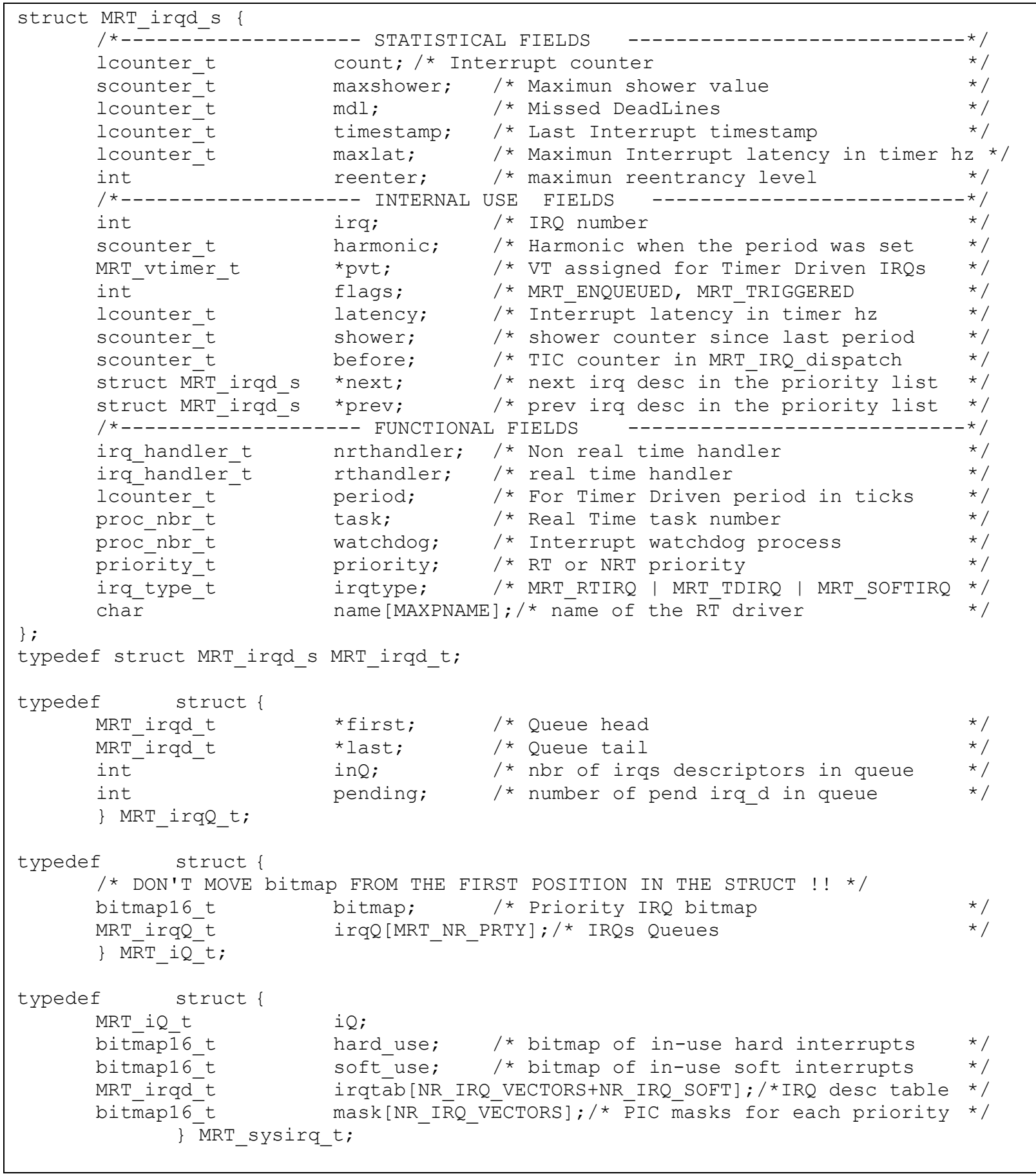




\section{D.2.3. Process-related Data Structures}

\section{Listing D.8: Process-related Data Structures.}

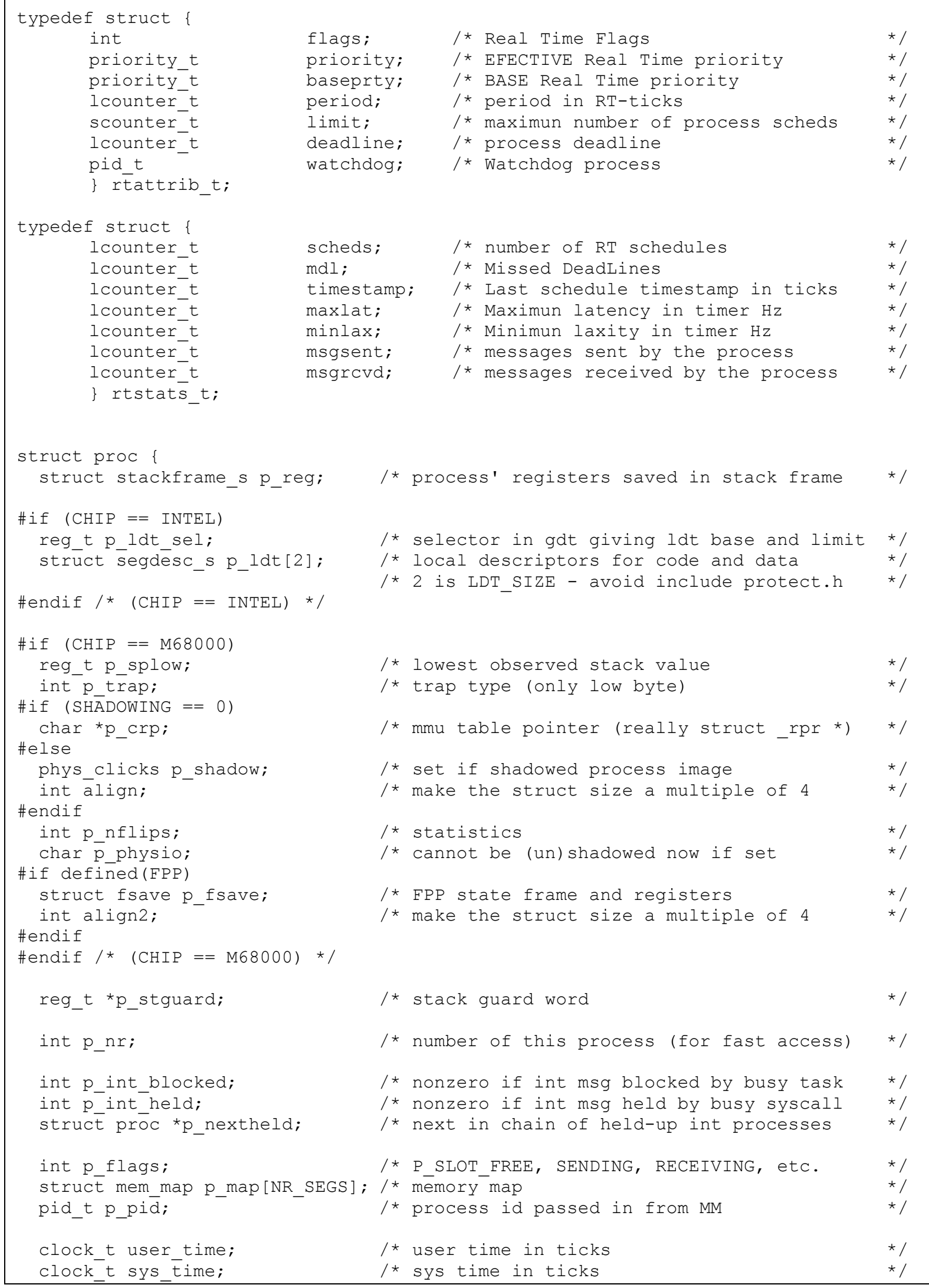




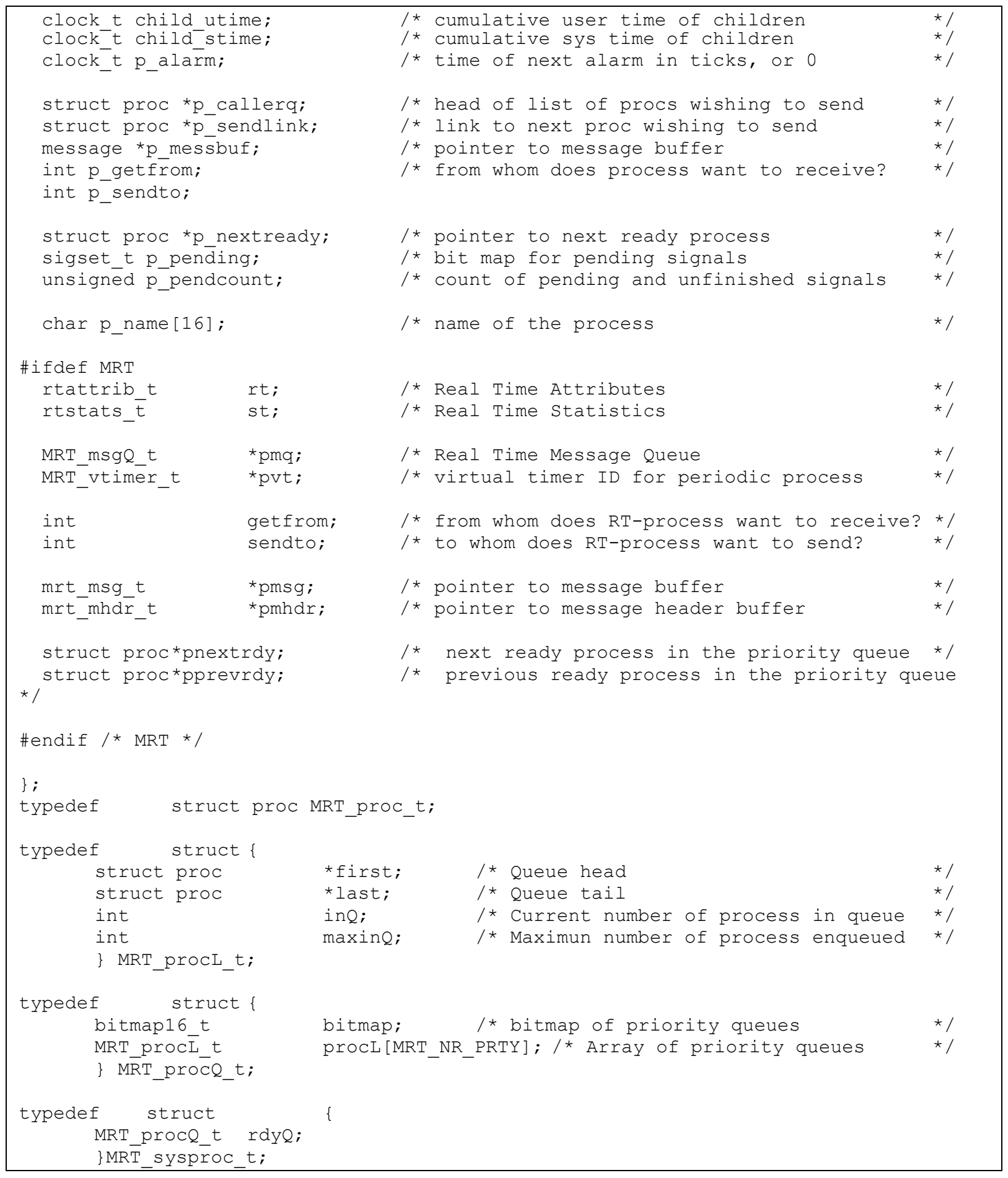

\section{D.2.4. Message-related Data Structures}

Listing D.9: Message-related Data Structures.

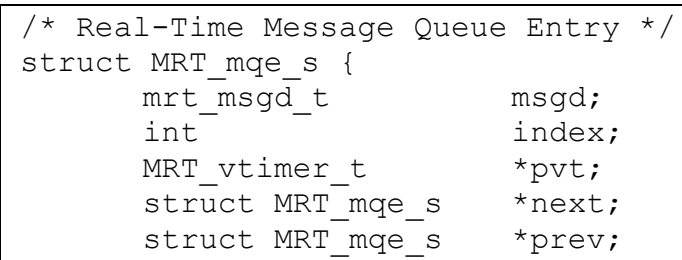




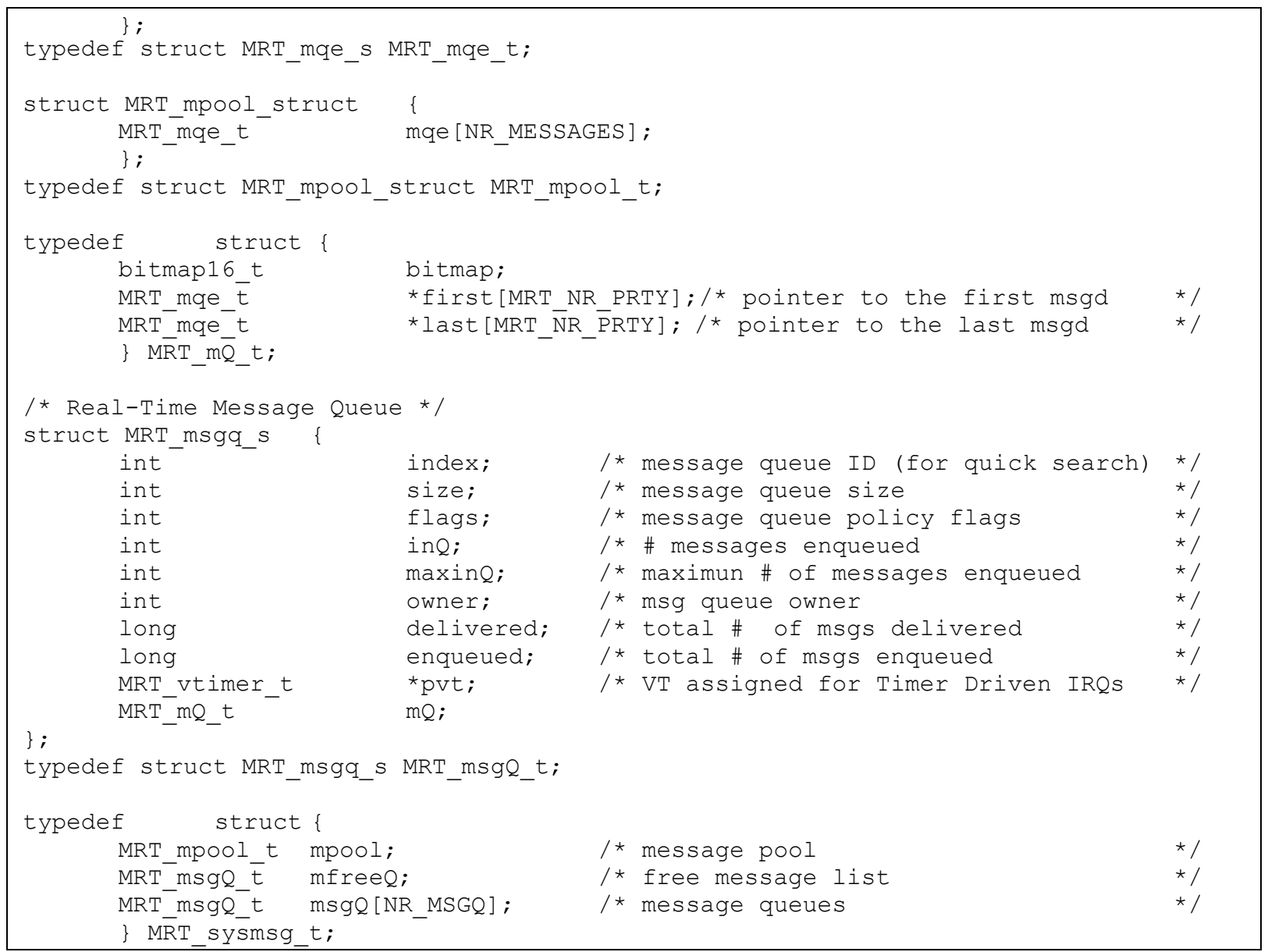




\section{GLOSARY}

Context

Context switching

CPU

CPU utilization

Critical region/section

Deadline

Deadline Monotonic Scheduling

Deadlock

Default

Device Driver
The minimum information that is needed in order to save a currently executing process so that it can be resumed.

The process of saving and restoring sufficient information for a Real-Time process so that it can be resumed after being interrupted.

Central Processing Unit.

A measure of the percentage of non Idle processing.

Code that interacts with a serially reusable resource.

A deadline is a point in time at which some operation must be completed.

Scheduling policy in Real-Time systems that meets a periodic process's deadline that does not equal its period.

A catastrophic situation that can arise when processes are competing forthe same set of two or more serially reusable resources.

The value or status that is assumed unless otherwise specified.

A program that translate Operating System mandated function calls into device specific calls 
Direct Memory Access (DMA)

Dynamic Real-time Scheduling Algorithm

Earliest-Deadline-First (EDF)

Embedded System

Event

Exception

Exception handler

First-In-First-Out (FIFO)

Hard Real-Time Scheduling

Hard Real-Time System

Host
A scheme in which access to the computer's memory is afforded to other devices in the system without the intervention of the CPU.

Scheduling algorithm that uses deadlines to assign priorities to processes throughout execution

Scheduling policy that gives a processor to the process with the closest deadline.

A computing machine contained in a device whose purposeis not to be a computer. For example, the computers in automobiles and household appliances are embedded computers. Embedded computers use embedded software, which integrates an operating system with specific drivers and application software. Their design often requires special software-hardware codesign methods for speed, low power, low cost, high testability, or other special requirements.

Any occurrence that results in a change in the state of a system.

Error or other special condition that arises during program execution.

Code used to process exceptions.

Nonpreemptive scheduling policy that dispatches processes according to their arrival time in the ready queue

Scheduling policy that ensures processes meet their deadlines.

A Real-Time system in which missing even one deadline results in system failure.

A computer that is the one responsible for performing a certain computation or function. 
Input and Output (I/O)

Interprocess Communications (IPC)

Interrupt

Interrupt Controller

Interrupt Handler

Interrupt Latency

Interrupt Service Routine (ISR)

Kernel

Kernel Calls

Latency (process scheduling)
Input/output, or $\mathrm{I} / \mathrm{O}$, is the collection of interfaces that different functional units (sub-systems) of an information processing system use to communicate with each other, or the signals (information) sent through those interfaces.

IPC is a set of techniques for the exchange of data between two or more threads in one or more processes.

An input to a processor that signals the occurrence of an asynchronous event. The processor's response to an interrupt is to save the current machine state and execute a predefined subprogram. The subprogram restores the machine state on exit and the processor continues in the original program.

A device that provides additional interrupt handling capability to a CPU.

A predefined subprogram that is executed when an interrupt occurs. The handler can perform input or output, save data, update pointers, or notify other processes of the event. The handler must return to the interrupted program with the machine state unchanged.

The delay between when an interrupt occurs and when theCPU begins reacting to it.

Piece of code that your processor executes when an external event, such as a timer, occurs.

The lowest portion of the operating system that provides for task scheduling, dispatching, and interprocess communication.

A kernel call is the mechanism used by an application program to request service from the operating system kernel Time a Process spends in a system mode before it is serviced. 
Laxity

\section{Least Slack First}

Library

Link

Linker

Make

Makefiles

Message Queue

Microkernel
Value determined by subtracting the sum of the current time and a process's remaining execution time from the process's deadline. This value decreases as a process nears its deadline.

It is a scheduling algorithm. It assigns priority based on the slack time of a process.

A set of precompiled routines that may be linked with a program at compile time or loaded at load time or dynamically at run time.

The portion of the compilation process in which separate modules are placed together and cross-module references resolved.

A computer program that takes one or more object files, assembles them into blocks that are to fit into particular regions in memory, and resolves all external (and possibly internal) references to other segments of a program and to libraries of precompiled program units.

Utility to maintain, update, and regenerate related programs and files. The commands to be executed are placed in a makefile.

Files that contain a collection of commands that allow groups of programs, object files, libraries, and so on, to interact. Makefiles are executed by your development system's make utility.

An interprocess communication facility consisting of a memory location and at least two basic operations send/receivethat can be performed on it.

A microkernel is a type of kernel which consists of defining a very simple abstraction over the hardware, with a set of primitives or system calls to implement minimal OS services such as thread management, address spaces and 
interprocess communication

Multitasking

Multitasking Operating System

Operating System

Policy

Predictability

Preempt

Preemptive Priority System

Primitives

Priority

Priority Inversion
The operation by which a microprocessor schedules the handling of multiple tasks or processes. In generated code, the number of tasks is equal to the number of sample times in your model.

An operating system that provides sufficient functionality to allow multiple programs to run on a single processor so that the illusion of simultaneity is created. Contrast with multiprocessing operating system.

A set of programs that manages the operations of a computer.It oversees the interaction between the hardware and the software and providesa set of services to system users.

Policy is a describing or proscribing set of rules and actions that encompass an ideal goal

A system whose timing behavior is always within an acceptable range

A condition that occurs when a higher-priority process interrupts a lower priority priority.

A system that uses preemption schemes instead of roundrobin or first-come, first-served scheduling.

Primitives are functions provided by the Operating System/Kernel as basic building blocks

Measure of a process's or thread's importance used to determine the order and duration of execution.

A condition that occurs because a lower priority process executes when a higher priority process is ready to run. 
Process

Process Control Block/Process Descriptor

Race Condition

Rate-Monotonic Scheduling

Ready State

Real-Time

Real-Time Computing

Real-Time System

Response Time
The context, consisting of allocated memory, open files, network connections, in which an operating system places a running program.

An area of memory containing information about the context of an executing program. Although the process control block is primarily a software mechanism used by the operating system for the control of system resources, some computers use a fixed set of process control blocks as a mechanism to hold the context of an interrupted process.

A situation where multiple processes access and manipulates hared data with the outcome dependent on the relative timing of these processes.

Is an optimal preemptive static-priority scheduling algorithm used in Real-Time operating systems

In the process control block model, the state of those processes that are ready to run, but are not running.

Refers to systems whose correctness depends not only on outputs bu the timeliness of those outputs. Failure to meet one or more of the deadlines can result in system failure.

Support for environments in which response time to an event must occur within a predetermined amount of time. Real-time systems may be categorized into hard, firm and, soft real time.

Computer that processes real-world events as they happen, under the constraint of a real-time clock, and that can implement algorithms in dedicated hardware. Examples include mobile telephones, test and measurement devices, and avionic and automotive control systems.

The time between the presentation of a set of inputs to a software system and the appearance of all the associated 
outputs.

Round-Robin Scheduling

Schedualability Analysis

Scheduler

Scheduling policy

Semaphore

Server

Stack

Synchronous

System Calls

System Implementation

Task

Throughput
Scheduling policy that permits each ready process to execute for at most one quantum per round. After the last process in the queue has executed once, the scheduler begins a new round by scheduling the first process in the queue,

The compile-time prediction of execution-time performance.

The part of the kernel that determines which task will run.

The way that the scheduler uses to determine which task runs by following a scheduling algorithm

A special variable or object type used for protecting critical regions.

A process used to manage multiple requests to a serially reusable resource.

A first-in, last-out data structure handle by some CPU instructions.

An operation or operations that synchronize processes.

A system call is the mechanism used by an application program to request service from the operating system

A phase of the software development life cycle during which a software product is integrated into its operational environment.

A special kind of process in MINIX that treats with devices A measure of the number of operation or transactions per second that can be processed. 
Time-Sharing

Timeslice

Timing Constraint

Trap

User Space

Watchdog
Time-sharing refers to sharing a computing resource among many users by multitasking.

Amount of time that a process is allowed to run on a processor before the process is preempted.

Time period during which a process (or subset of a process's instructions) must complete

Internal interrupt caused by the execution of a certain instruction.

Memory not required by the operating system.

A process that is scheduled or signaled when something in the system behavior is wrong. 